San Jose State University

SJSU ScholarWorks

Master's Theses

Master's Theses and Graduate Research

Summer 2013

\title{
Titan I Propulsion System Modeling and Possible Performance Improvements
}

Oreste Giusti

San Jose State University

Follow this and additional works at: https://scholarworks.sjsu.edu/etd_theses

\section{Recommended Citation}

Giusti, Oreste, "Titan I Propulsion System Modeling and Possible Performance Improvements" (2013). Master's Theses. 4339.

DOI: https://doi.org/10.31979/etd.hsrk-f44z

https://scholarworks.sjsu.edu/etd_theses/4339

This Thesis is brought to you for free and open access by the Master's Theses and Graduate Research at SJSU ScholarWorks. It has been accepted for inclusion in Master's Theses by an authorized administrator of SJSU ScholarWorks. For more information, please contact scholarworks@sjsu.edu. 


\title{
TITAN I PROPULSION SYSTEM MODELING
}

\section{AND POSSIBLE PERFORMANCE IMPROVEMENTS}

\author{
A Thesis \\ Presented to \\ San José State University \\ In Partial Fulfillment \\ of the Requirements for the Degree \\ Master of Science
}

The Faculty of the Department of Mechanical and Aerospace Engineering

by

Oreste Giusti

August 2013 
(C) 2013

Oreste Giusti

\section{ALL RIGHTS RESERVED}


The Designated Thesis Committee Approves the Thesis Titled TITAN I PROPULSION SYSTEM MODELING AND POSSIBLE PERFORMANCE IMPROVEMENTS

by

Oreste Giusti

APPROVED FOR THE DEPARTMENT OF MECHANICAL AND AEROSPACE ENGINEERING

\section{SAN JOSÉ STATE UNIVERSITY}

August 2013

Dr. Periklis Papadopoulos, Department of Mechanical, and Aerospace Engineering

Dr. Nikos Mourtos, Department of Mechanical, and Aerospace Engineering

Prof. Marc Murbach, Department of Mechanical, and Aerospace Engineering 


\section{ABSTRACT \\ TITAN I PROPULSION SYSTEM MODELING \\ AND POSSIBLE PERFORMANCE IMPROVEMENTS}

by

Oreste Giusti

This thesis features the Titan I propulsion systems and offers data-supported suggestions for improvements to increase performance. The original propulsion systems were modeled both graphically in CAD and via equations. Due to the limited availability of published information, it was necessary to create a more detailed, secondary set of models. Various engineering equations--pertinent to rocket engine design--were implemented in order to generate the desired extra detail. This study describes how these new models were then imported into the ESI CFD Suite. Various parameters are applied to these imported models as inputs that include, for example, bi-propellant combinations, pressure, temperatures, and mass flow rates. The results were then processed with ESI VIEW, which is virtualization software. The output files were analyzed for forces in the nozzle, and various results were generated, including sea level thrust and ISP. Experimental data are provided to compare the original engine configuration models to the derivative suggested improvement models. 


\section{ACKNOWLEDGEMENTS}

I would like to thank Dr. Periklis Papadopoulos, Dr. Nikos Mourtos, and Dr. Marc Murbach for their support and guidance throughout the process of this study. I would also like to thank Ben Nikaido for his support with the ESI computational fluid dynamic code. Also, I would like to thank my family for their unending confidence in my abilities, my close friends for their continued interest, and my fellow classmates for their moral support every Saturday. I would also like to thank my fellow Titan I researcher, Justin Rothenberg, for his joint perseverance on the long road to our theses. I would also like to thank the San José State University School of Music and Dance for providing me with financial stability and a cool and quiet work space, both essential to the completion of this work. Finally, and most importantly, I would like to thank my wife, Marylin Giusti, for her diligence in keeping me fed, healthy, and grammatically consistent, all pivotal for the most involved work I have completed to date. 


\section{TABLE OF CONTENTS}

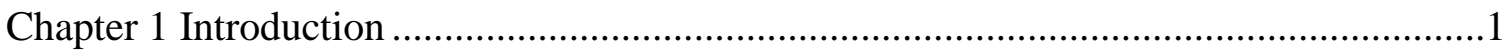

Motivation and Objectives ................................................................................ 1

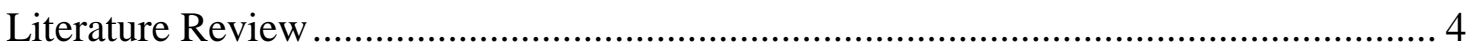

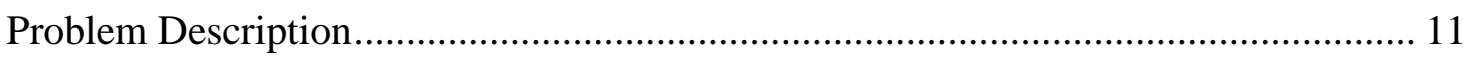

Chapter 2 Computational Method Description .......................................................... 15

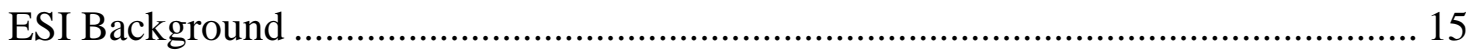

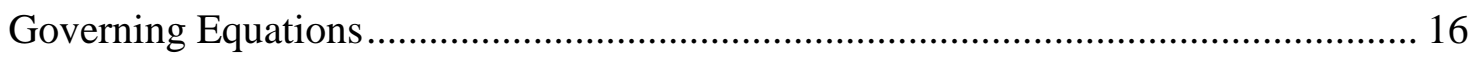

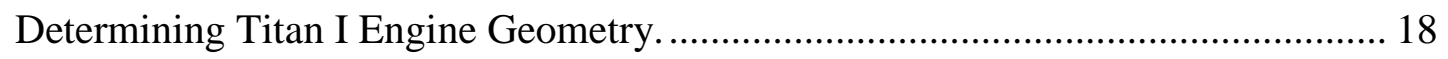

Computational Fluid Dynamics equations used in ESI FASTRAN SOLVER. ........ 29

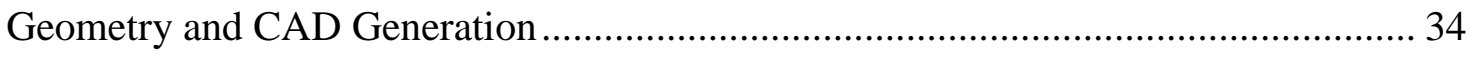

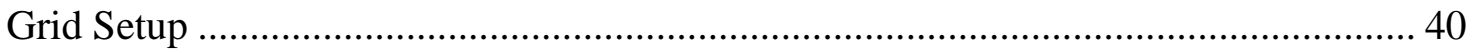

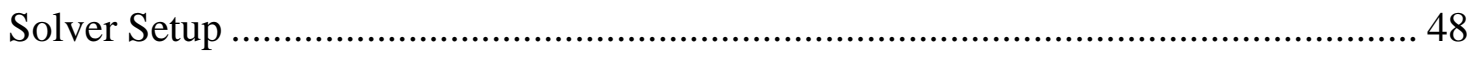

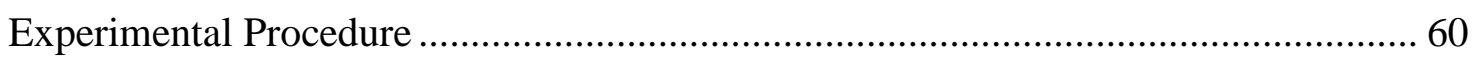

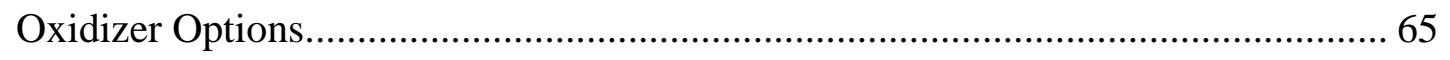

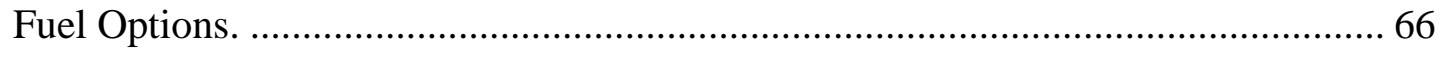

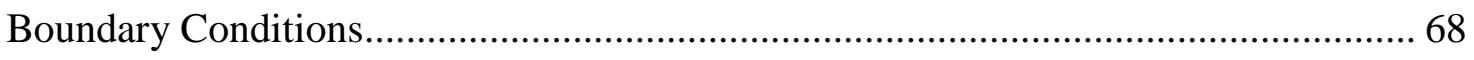

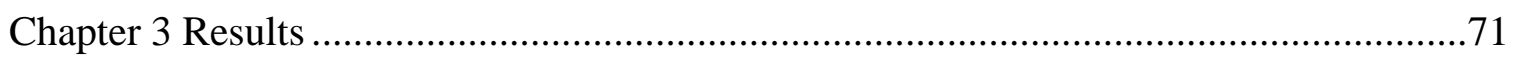

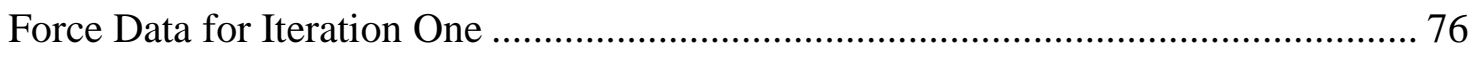




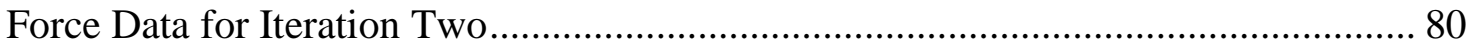

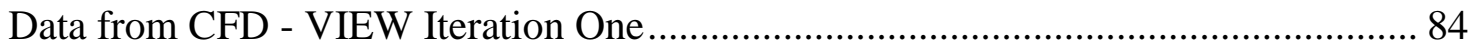

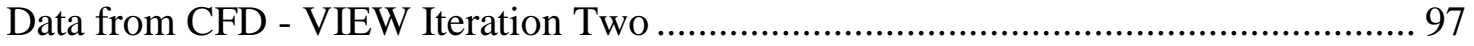

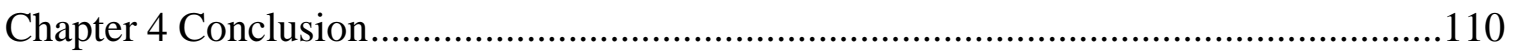

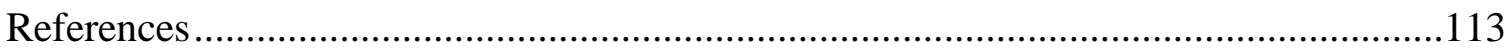

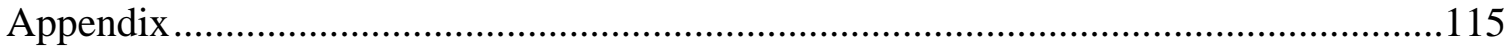

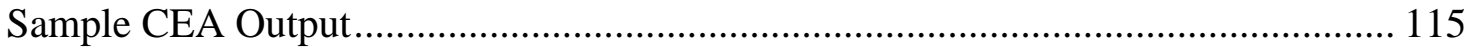

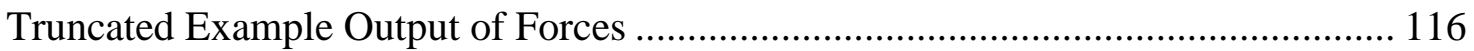

EXCEL Spreadsheet Calculator Example for a Stage One Setup........................... 117 


\section{TABLE OF FIGURES}

Figure 1 X-20A Dyna-Soar on Titan I Booster ........................................................ 4

Figure 2 Regenerative Coolant Flow .................................................................... 7

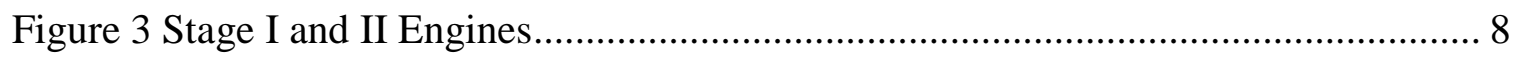

Figure 4 Gas Generator Cycle ("Gas generator rocket," 2008) ................................... 10

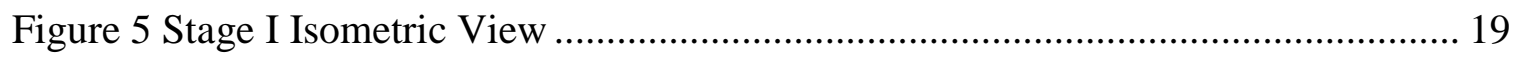

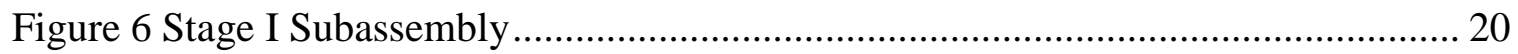

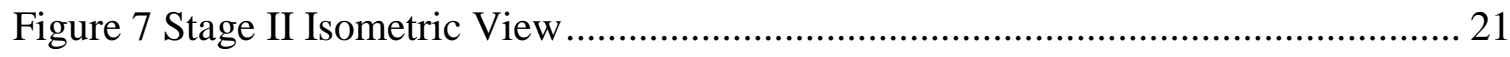

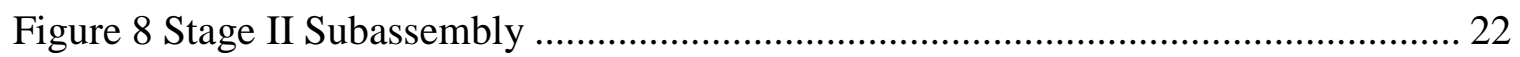

Figure 9 3D CAD Models (Not to Scale) derived from Figure 2 Schematic .................. 23

Figure 10 EXCEL of Stage I Geometry Values.................................................... 24

Figure 11 Parameterized Nozzle Design (Rao Method) ............................................ 27

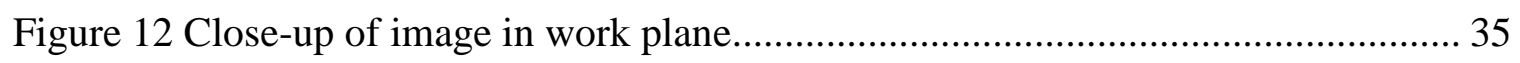

Figure 13 Outline of CAD Model on Schematic ................................................... 36

Figure 14 Final step in the model creation procedure ................................................ 37

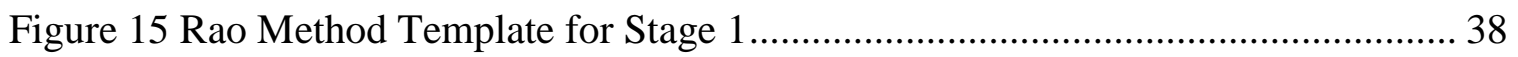

Figure 16 Stage I CAD Model from Geometric Calculations ..................................... 39

Figure 17 Stage I CAD Model from Geometric Calculations ...................................... 39

Figure 18 Imported View of the Geometry from Solidworks.................................... 41

Figure 19 Close-up of Boundary Layer of Stage One Grid ....................................... 42

Figure 20 Imported Geometry Edge with Grid Points ............................................. 43

Figure 21 Grid Application to Nozzle Zone .................................................................. 44

viii 
Figure 22 Whole View of Boundary Layer of Stage 1 ........................................... 45

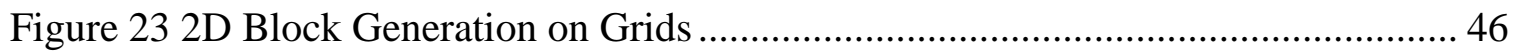

Figure 24 Selecting Boundary Condition Section Types ........................................ 47

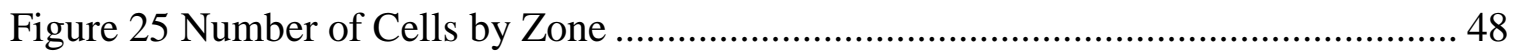

Figure 26 MO tab display both the Global Options and Flow Selections ...................... 51

Figure 27 VC Module with Fluid Properties Selection ............................................. 53

Figure 28 Boundary Conditions of the model.......................................................... 54

Figure 29 Inputs for the Inlet Boundary Condition Subtype .................................... 55

Figure 30 Example for setting of the Initial Boundary Conditions .............................. 56

Figure 31 The sub-tabs under the SC tab that were modified..................................... 58

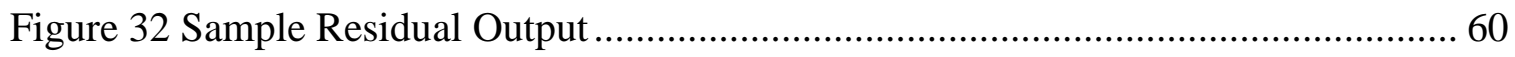

Figure 33 Stage One Differences in the Contours ................................................. 72

Figure 34 Stage Two Differences in the Contours..................................................... 72

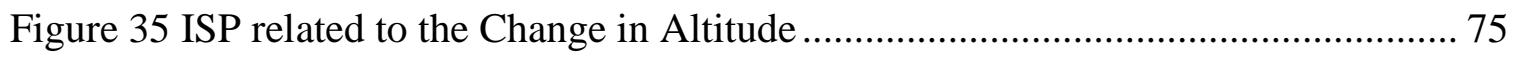

Figure 36 ISP compared to Thrust for Stage I for Iteration One ................................ 78

Figure 37 ISP Compared to Thrust for Stage II for Iteration One ................................ 79

Figure 38 ISP Compared to Thrust for Stage I Iteration Two …................................ 82

Figure 39 ISP Compared to Thrust for Stage I Iteration Two ..................................... 83

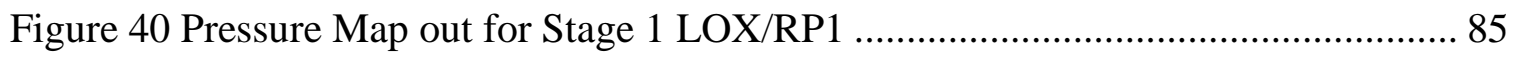

Figure 41 Temperature Map Output for Stage 1 LOX/RP1 ....................................... 85

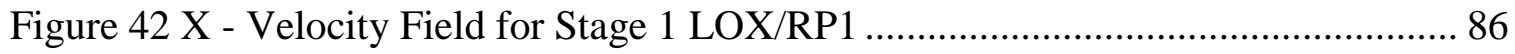

Figure 43 Mach Zones Output for Stage 1 LOX/RP1 ................................................. 86 
Figure 44 Pressure Map Output for Stage 1 LOX/LH2 ........................................... 87

Figure 45 Temperature Map Output for Stage 1 LOX/LH2 ..................................... 87

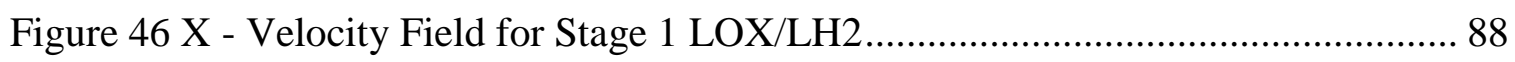

Figure 47 Mach Zone Output for Stage 1 LOX/LH2 ................................................. 88

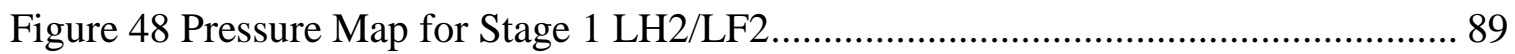

Figure 49 Temperature Map for Stage 1 LH2/LF2 ................................................ 89

Figure 50 X - Velocity Field for Stage 1 LH2/LF2 _.................................................. 90

Figure 51 Mach Zones Output for Stage 1 LH2/LF2 ............................................. 90

Figure 52 Pressure Map Output Stage 1 LOX/RP1 ................................................. 91

Figure 53 Temperature Map Output for Stage 1 LOX/RP1 ..................................... 91

Figure 54 X - Direction Velocity Field for Stage 1 LOX/RP1 .................................... 92

Figure 55 Mach Zones Output for Stage 1 LOX/RP1 ............................................. 92

Figure 56 Pressure Map Output for Stage 2 LOX/LH2 ......................................... 93

Figure 57 Temperature Map Output for Stage 2 LOX/LH2 .................................... 93

Figure 58 X - Velocity Field Output for Stage 2 LOX/LH2 ...................................... 94

Figure 59 Mach Zones Output for Stage 2 LOX/LH2 ….......................................... 94

Figure 60 Pressure Map Output for Stage 2 LF2/LH2 …........................................ 95

Figure 61 Temperature Map Output for Stage 2 LF2/LH2 ....................................... 95

Figure 62 X - Velocity Field for Stage 2 LF2/LH2 ............................................... 96

Figure 63 Mach Zones Output for Stage 2 LF2/LH2 ............................................... 96

Figure 64 Pressure Map out for Stage 1 LOX/RP1 .................................................. 98

Figure 65 Temperature Map Output for Stage 1 LOX/RP1 ......................................... 98 
Figure 66 X - Velocity Field for Stage 1 LOX/RP1 ………………............................... 99

Figure 67 Mach Zones Output for Stage 1 LOX/RP1 ................................................... 99

Figure 68 Pressure Map Output for Stage 1 LOX/LH2 ............................................. 100

Figure 69 Temperature Map Output for Stage 1 LOX/LH2 ......................................... 100

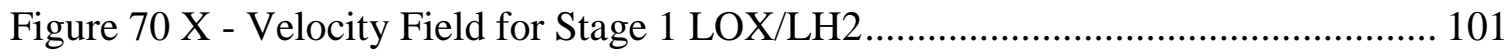

Figure 71 Mach Zones Output for Stage 1 LOX/LH2 .................................................. 101

Figure 72 Pressure Map Output for Stage 1 LH2/LF2 …………………………...... 102

Figure 73 Temperature Map Output for Stage 1 LH2/LF2 ......................................... 102

Figure 74 X - Velocity Field for Stage 1 LH2/LF2 ………....................................... 103

Figure 75 Mach Zones Output for Stage 1 LH2/LF2 ……………………………...... 103

Figure 76 Pressure Map Output for Stage 2 LOX/RP1 .............................................. 104

Figure 77 Temperature Map Output for Stage 2 LOX/RP1 .......................................... 104

Figure 78 X - Velocity Field Output for Stage 2 LOX/RP1 …………………………... 105

Figure 79 Mach Zones Output for Stage 2 LOX/RP1 ................................................ 105

Figure 80 Pressure Map Output for Stage 2 LOX/LH2 .............................................. 106

Figure 81 Temperature Map Output for Stage 2 LOX/LH2 ……………………….... 106

Figure 82 X - Direction Velocity Field Output for Stage 2 LOX/LH2 ……………..... 107

Figure 83 Mach Zones Output for Stage 2 LOX/LH2 ................................................ 107

Figure 84 Pressure Map Output for Stage 2 LH2/LF2 …............................................. 108

Figure 85 Temperature Map Output for Stage 2 LH2/LF2......................................... 108

Figure 86 X - Direction Flow Field Output for Stage 2 LH2/LF2 ................................. 109

Figure 87 Mach Zones Output for Stage 2 LH2/LF2 …………………………........ 109 


\section{LIST OF TABLES}

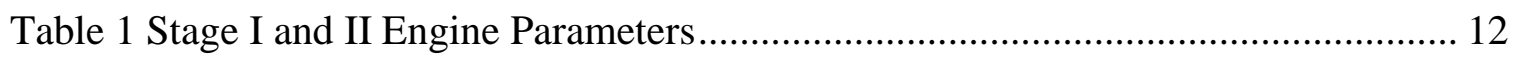

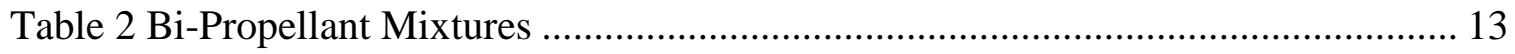

Table 3 Table of Inputs and Parameters for Iteration One........................................ 61

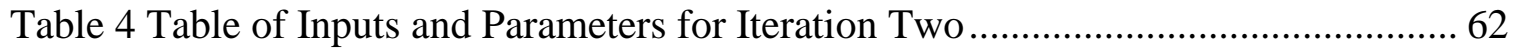

Table 5 Propellant Data at Normal Conditions (Huzel \& Huang, 1992)......................... 64

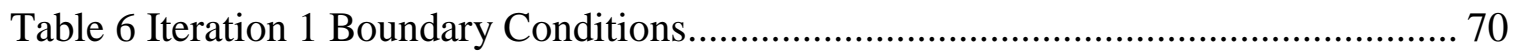

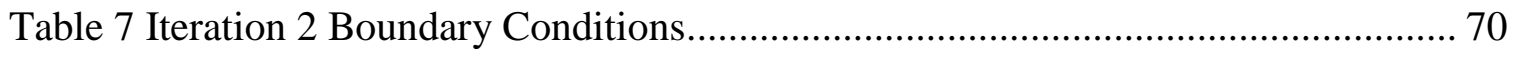

Table 8 Comparison Between Published and Calculated Data...................................... 73

Table 9 Percent Difference Between Published and Calculated Data ............................ 74

Table 10 Total Forces and Moments on Each Patch of Wall BC for Iteration One ........ 76

Table 11 Calculated Thrust and ISP from CFD Data using Iteration One Inputs ............ 77

Table 12 Percent Difference Comparison with Published Data for Table Above........... 79

Table 13 Total Forces and Moments on Each Patch of Wall BC for Iteration Two......... 80

Table 14 Calculated Thrust and ISP from CFD Data using Iteration Two Inputs........... 81

Table 15Percent Difference Comparison with Published Data for Table above .............. 83

Table 16 Titan I Proposed Improvement Parameters ............................................... 112 


\section{Chapter 1 Introduction}

\section{Motivation and Objectives}

The motivation behind this work was to study the potential use of the Titan I as a starting point for the design of a commercial launch vehicle and a general curiosity of early propulsion history. The objective of this thesis was to explore and understand the propulsion systems of the Titan I launch vehicle. During this process possible propulsion system updates to the engine characteristics were considered.

The Titan family of launch vehicles is an intriguing and important evolutionary stepping stone to the modern and complex launch-delivery systems that we have today because it marks the start of large scale, liquid, bi-propellant rocket vehicles. Originally designed to carry and deliver nuclear warheads, this vehicle was at the forefront of Cold War nuclear deployment technology. The system was soon replaced due to its limited launch capabilities, particularly the time required for fueling before launch countdown. Not only was the turnaround time for launch a setback, but the limited payload of 1800 $\mathrm{kg}$ also contributed to its retirement as newer vehicles could carry larger payloads.

Currently, a variety of commercial launch vehicles exist that are used to service the small payload launch niche market. This market has a requirement for on-demand small launchers, and the market has a variety of customers that range from government organizations to private research. The vehicles used in this market are varied and include the Dnepr-1, Falcon 1e, Minotaur IV, and the newly launched Vega among a few. All of 
these vehicles have a payload capacity to low earth orbit (LEO) of $2500 \mathrm{~kg}$ or less (Isakowitz et al., 2004). The launch costs of these vehicles varies, but currently the cheapest on the market is the Falcon 1e ("Falcon 1 overview," 2012). It can be seen from comparison that Titan I is not far off from modern standards for launch vehicles required for the small launcher market when compared to the previously mentioned vehicles.

Due to the fact that the Titan I has a payload to orbit capacity of $1800 \mathrm{~kg}$ on a liquid bipropellant system that operates on oxygen and kerosene the system demonstrates a platform with a potential for growth. This is because that the materials used in the design of the propulsion system allow the design to be minimally altered to use other propellants. This vehicle then becomes attractive because of its innate ability for versatility. Alternative propellants that could be used to increase different aspects of performance include the hypergolic mixture of nitrogen tetra-oxide and Aerozine used in the subsequent Titan II. Other potential mixtures that could offer increased performance include liquid fluorine and hydrogen as well as the Space Shuttle Main Engine mixture of liquid oxygen and hydrogen.

In order to accomplish any study of performance increase, the initial geometry of the system must be known and hence these values will need to found through a variety of methods. These methods include researching time era documents and basic reverse engineering through the manipulation of known design equations by using published engine characteristics as the starting point. This allows for a greater understanding of the early engine designs and aids in expanding the knowledge base of aerospace engineering by reporting on difficult to find or lost geometric layouts for the engines. This 
information could then be used to identify the viability of reusing old systems with minor modifications without the need to create new support infrastructure for manufacturing thereby reducing the time required to develop and create a flight vehicle for specific missions.

Increasing propulsive efficiency, coupled with minimal refurbishment of the structure that could take advantage of modern manufacturing techniques and materials could increase the performance and ease of production of the Titan I. The vehicle's propulsion systems could also take advantage of computational fluid dynamics (CFD) research to increase efficiency and help design a fast process for quick prototyping during the redesign phase. Another important and interesting fact is that the Titan I at one point was considered as a possible launch vehicle to the cancelled single pilot space plane known as the X-20A Dyna-soar by Boeing (Houchin, 2006). It is clear based on the vehicles history that with proper modifications and updates to the propulsion system one could increase the economic viability of using this vehicle for small payload insertion missions. Figure 1 displays an artist's rendition of how the Dyna-Soar may have looked like atop a Titan I launch vehicle (NASA, 1961). This clearly demonstrates another possible use for the Titan I. 


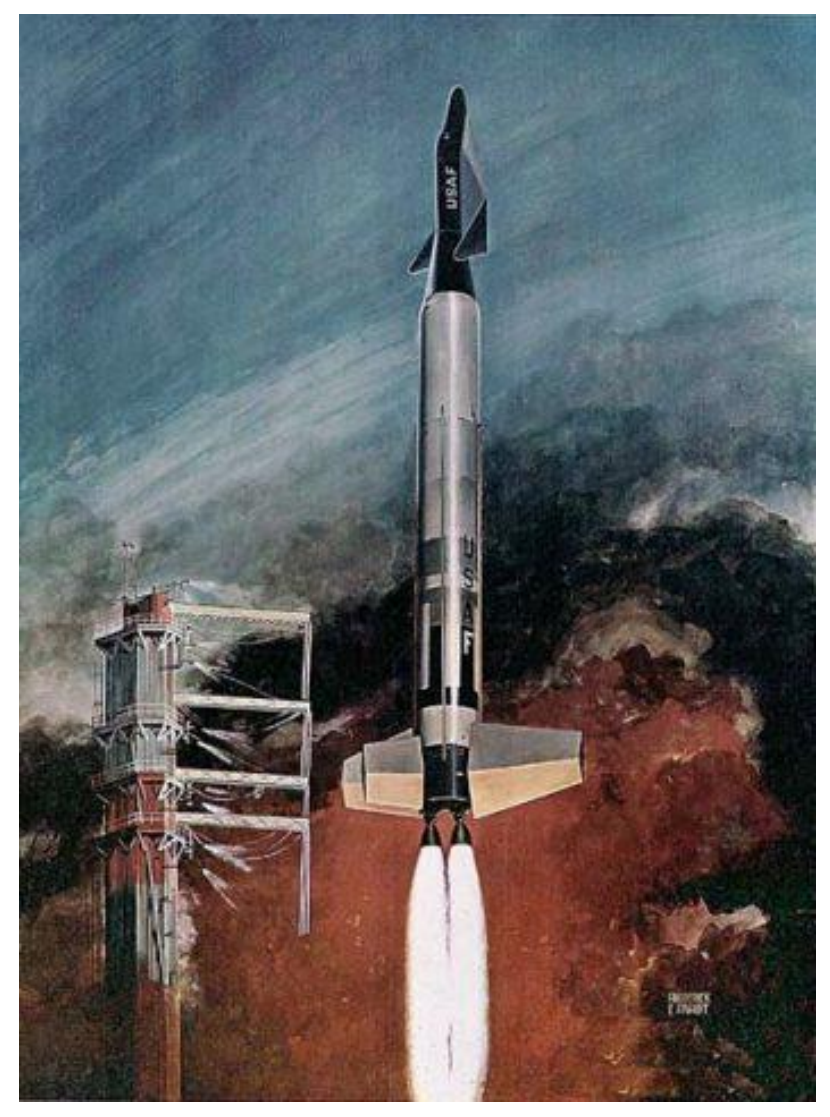

Figure 1 X-20A Dyna-Soar on Titan I Booster

\section{Literature Review}

The Titan I launch vehicle was designed and constructed by the Glenn L. Martin Company, now known as the Lockheed Martin Company, with the propulsion system designed and manufactured by Aerojet TechSystems (Launius, 2002). The Titan I was known originally as the WS 107A-2 and was designed as a backup system to the famous Atlas launch vehicle in 1955 and was described as the nations "insurance" to the Atlas missile program (Hunley, 2007). Prior to the Titan I there had been a long history of liquid rocket technology development, which began with the United States conducting involved research on liberated short range German V-2 rockets. This eventually led to 
the development of the Viking sounding rocket and other early rocket vehicles (Adams, 1990). During the post-World War II, era great emphasis was placed on intercontinental ballistic missile development (ICBM) to help deploy the United States growing nuclear arsenal. This would eventually lead to the development of the Atlas family of vehicles, which would culminate to the SM-65A Atlas until the Titan I was considered as a second choice.

The intent of the Titan I was to design a vehicle that used many of the same materials, such as copper rich Aluminum 2014 and phenolic materials, and parts of the Atlas launch vehicle in order to reduce spare part shortages during protracted engagements, maintenance, and upkeep for the Air Force (Lange, 1963). Another reason for its development was that it was used to rapidly generate competitive growth in development. The Titan I is known to be the nation's first two stage ICBM, because prior to the Titan I, the Atlas vehicles used a $1 \frac{1}{12} 2$ stage configuration (Launius, 2002). The $1 \frac{1}{2}$ stage configuration consisted of igniting both the first and second stage engines on the launch pad prior to launch as a means to ensure that the upper stage would detach from the first. This was deemed to be a safer approach for mission success than igniting the second stage during flight.

The Titan I paved the way for a true two stage vehicle by being able to ignite its second stage engine in flight after separating from its first stage via the use of small solid rockets attached to the second stage (Adams, 1990). The separation method also made use of staging rails, which would guide the upper stage away from the lower stage vehicle. Once the upper stage was away vernier roll control nozzles would help guide the 
upper stage to the intended target (Meland, 1989). One of the innovative design features implemented on the full scale production model of the Titan I was the use of a bipropellant rocket propulsion system that utilized liquid oxygen and refined petroleum - 1 (RP-1). The engines of the two stages were known as the LR87-A5-1 and the LR-91-AJ1 , respectively, and were both regeneratively cooled.

Regenerative cooling is when a propulsion system uses one of the propellants, usually the fuel, to cool the exterior of the combustion chamber and nozzle. This is done with a creative application of fine tubing or channels directly on the surface of the engine. The system allows for high velocities of coolant around the throat area, which typically is the zone with the highest amount of heat. The intent is to reduce the temperature of the material to mitigate damage or failure while heating the propellant to be used either directly into combustion or dumping it out for added cooling. The Titan family of engines makes use of double-pass regenerative cooling. This cooling method is when the fuel is routed down from the injector head, collected in a manifold, and then routed back around (Brown, 1996). The system has some disadvantages, the first being that it can add to the total engine mass, complexity of the plumbing, and require compatible pumps. The advantages include the ability to run the engine for longer periods of time, more flexibility with materials, and weight savings from the reduction of ablative materials. Figure 2 displays regenerative coolant flow for an example propulsion system. The blue line denotes the fuel and demonstrates how it cools the outside walls and is then recirculated back into the combustion chamber. 


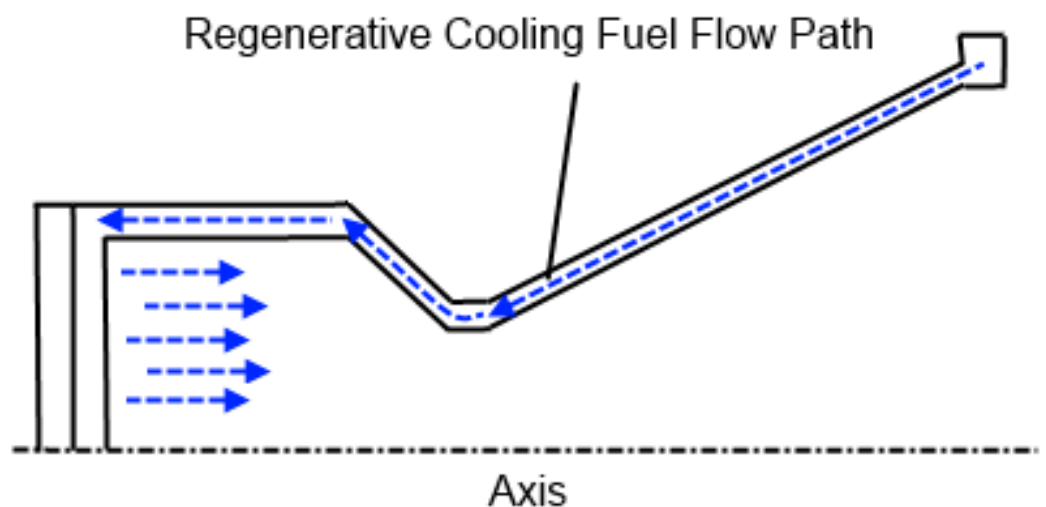

Figure 2 Regenerative Coolant Flow

The LR87 had an operational time of $140 \mathrm{~s}$ while the LR91 had an operational time of $150 \mathrm{~s}$ and both sported ablative skirts. The LR87 was designed to operate near sea level conditions while the upper stage LR91 was designed to operate in the upper atmosphere. The vehicle was designed to deliver a payload of about $3800 \mathrm{lb}(1800 \mathrm{~kg})$ with a range of 5500 nautical miles (Adams, 1990). The total mass of the vehicle was $220,000 \mathrm{lb}$ of which about $90 \%$ consisted of fuel. The first stage of the vehicle was about 57 feet long with a diameter of 10 feet and the second stage was 31 feet long with a diameter of 8 feet (Adams, 1990). The performance characteristics are displayed below in Figure 3 Stage I and II Engines (Reber, 1986). It is important to note that these performance characteristics are the published values allowed for use in the public domain. They certainly do not represent any actual flight vehicle values or even experimental setups. These values most likely come from assumed or generalized results. 
They can be near the actual values, but without any actual data from the time of testing this is uncertain to know or ascertain.

Stage I
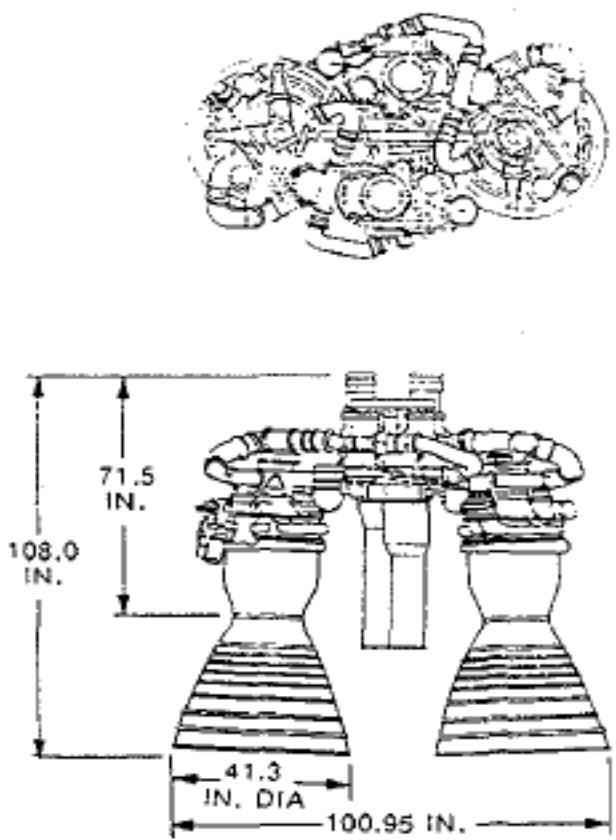

Stage II
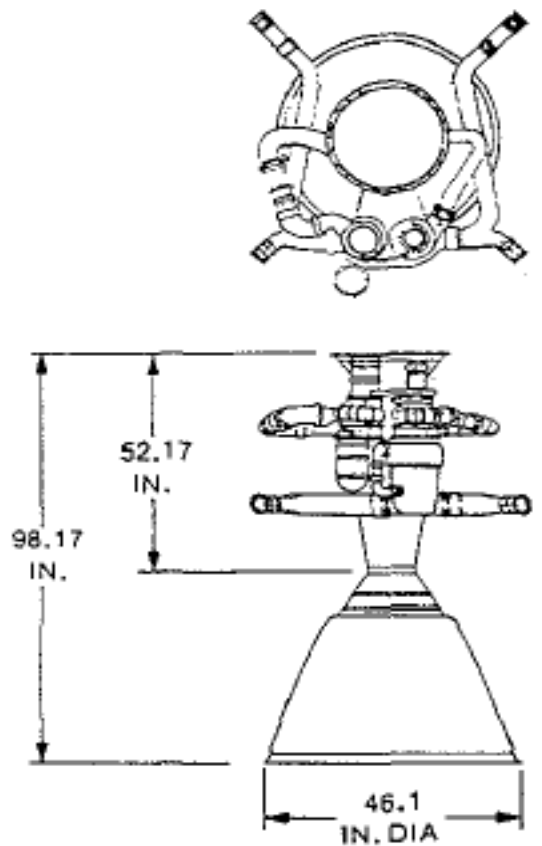

Sea Level Thrust (Ib)

Altitude Thrust (lb)

Number of Engines

Engine Cycle

Propellants, O/F

Mixture Ratio, $\mathbf{O} / \mathbf{F}$

Chamber Pressure, psia

Area Throat, $\ln ^{2}$

Area Ratio, Ae/At

Sea Level Isp (sec)

Altitude Isp (sec)

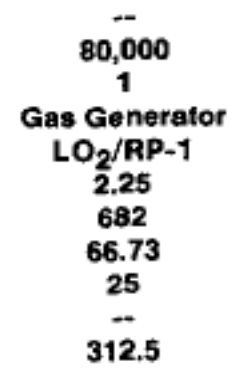

Figure 3 Stage I and II Engines. Reprinted with permission of the American

Institute of Aeronautics and Astronautics.

As can be seen in Figure 3 the performance of the engines was relatively high compared to modern standards of liquid bi-propellant rocket engines. Other 
contemporary systems such as the SM-65 Atlas had a smaller payload capacity as well as limited capabilities due to the fact that it was not a true two stage vehicle and therefore heavier. The effectiveness of the Titan I was partly due to the propellant choices and the overall design of the engines. The engines were designed to utilize a gas generator cycle in order to help power the turbo-pumps that fed propellants to the engines while the tanks were pressurized with an auxiliary helium pressurant tank in order to create the positive pressure required for the pumps (Meland, 1989). A gas generator system uses some of the propellant gas that is created from the burned reaction to power the engines pump. The gas used to power the pumps is then exhausted, hence an extra "exhaust pipe" between the engines of the Titan I first stage engines exist, which can be seen in Figure 3 . The gas generator cycle is also the only of three cycles that has the turbine flow path in parallel with the thrust chamber flow path making this system simpler to design and operate (Huzel \& Huang, 1992). Figure 4 displays what a gas generator cycle system looks like and how it functions. 


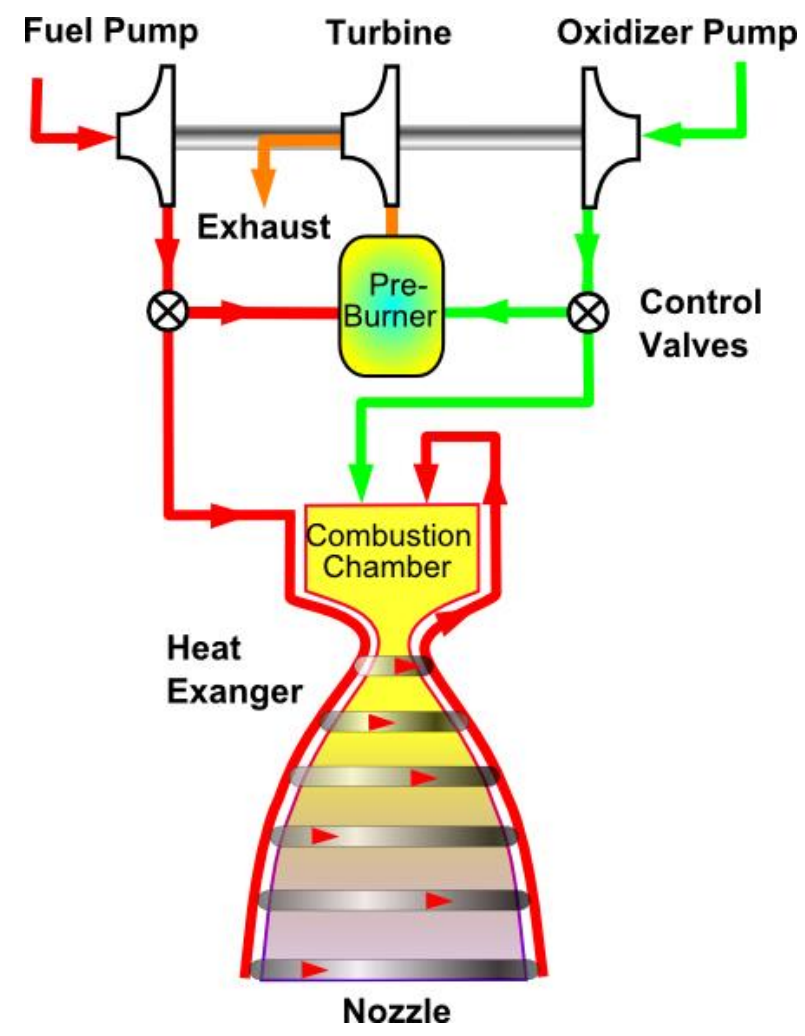

Figure 4 Gas Generator Cycle ("Gas generator rocket," 2008)

A gas generator system has both advantages and disadvantages. The advantages to this system is that it reduces the plumbing required for the system thus reduces the overall mass of the propulsion unit and raises its efficiency in the thrust to weight ratio and complexity categories. The main disadvantage of the gas generator cycle system is that it can create soot and coking with carbon-bearing fuels that can adversely affect propulsion system thereby causing performance losses because of the injectors becoming clogged (Huzel \& Huang, 1992). This disadvantage can be mitigated through design and or altering the propellant choices to a non-carbon based fuel.

Although revolutionary the Titan I vehicle had a short operational life span from 1962 to 1965 before it was deactivated. This was due to the fact that the vehicle required 
15 minutes minimum to load its propellants before a launch which greatly impeded its use as a quick strike weapon (Hunley, 2007). The second iteration of the Titan model known as the Titan II was done as an attempt to solve this problem by switching to hypergolic fuels for quicker fueling and turnaround time for launch (Hunley, 2007). The Titan I was deployed in various locations around the United States in three-missile battery complexes.

\section{Problem Description}

This study mainly focused on modeling the Titan I propulsion systems. This study analyzed the flow of different combusting reactant mixtures from the combustor face to the end of the nozzle of the propulsion systems of both stage one and two of the Titan I. This study also focused on attempting to accurately represent the original Titan I propulsion system from limited sources in a 3D CAD environment. Due to the severe limitation on published design parameters a second model of the systems was generated through the implementation of various engineering calculations. The modeling consisted of generating a geometric layout of both engines on the Titan I from the values found in Figure 3 and displayed for convenience in Table 1 below. Two sets of models were generated and compared by finding the percent difference between them. 


\section{Table 1 Stage I and II Engine Parameters}

\begin{tabular}{|c|c|c|}
\hline \multicolumn{2}{|c|}{ Stage I Engine } & \multicolumn{2}{c|}{ Stage II Engine } \\
\hline Value & Parameter & Value \\
\hline 300,000 & Sea Level Thrust (lb) & -- \\
\hline 344,400 & Altitude Thrust (lb) & 80,000 \\
\hline 2 & Number of Engines & 1 \\
\hline 2.25 & Mixture Ratio (O/F) & 2.25 \\
\hline 587 & Chamber Pressure, psia & 682 \\
\hline 181.9 & Area Throat, in & 66.73 \\
\hline 8 & Area Ratio, Ae/At & 25 \\
\hline 251.9 & Sea Level Isp (sec) & -- \\
\hline 289.1 & Altitude Isp (sec) & 312.5 \\
\hline
\end{tabular}

The purpose of creating two models for the propulsion systems was to determine

the difference between the two in order to see how close the calculated values compare to the published values. Creating the benchmark helped determine how accurate the original drawing was to the performance it can generate. This was determined by comparing the generated geometric values of the Titan I system to the traced blueprint design that was found.

Once the dimensioning of the original engine is completed CFD runs were conducted on the propulsion systems to determine what the flow field results would be based on the dimensions generated through engineering calculations. The CFD program used was the ESI CFD Suite and was used to generate the required mesh as well as the grid that was needed for the application of the test. A basic grid and geometry was input into the FASTRAN code at this point the initial set of runs was begun. The initial set of data to be input into FASTRAN will initially focus on the original bi-propellant mixture to generate a benchmark with published data. This information will then be used in combination with the determined geometric results to setup a benchmark for the original 
propulsion systems. This benchmark will then be used towards determining viable performance improvements that will include modifying parameters such as pressure and propellant mixtures.

The generated geometric values were determined by applying well known engineering equations for the design of bi-propellant rocket engines found in various texts. It will also allow the geometry to be easily changed in order to increase or decrease performance to within an acceptable range to the published data. The procedure that will handle this process will take into consideration modifying the chamber pressures, mixture ratio, area of the throat, and area ratio.

The data derived through the completion of this problem will focus on factors such as sea level thrust as well as specific impulse (ISP). Then once this set of data is established the engines was tested keeping all else the same, but modifying the bipropellant mixtures. The aim is to generate a table to compare and contrast the positive and negative aspects of the different bi-propellants used with the geometry of the Titan I propulsion system. Furthermore, an improvement in terms of increasing the chamber pressures of both the first and second stage engines will also be considered. The different bi-propellant combinations to be explored are displayed in Table 2 below.

Table 2 Bi-Propellant Mixtures

\begin{tabular}{|l|l|}
\hline \multicolumn{1}{|c|}{ Oxidizer } & \multicolumn{1}{c|}{ Fuel } \\
\hline Liquid Oxygen & Kerosene \\
\hline Liquid Oxygen & Hydrogen \\
\hline Fluorine & Hydrogen \\
\hline
\end{tabular}


The limitation of this work can be divided into three categories: time, resources, and technology. These three factors are tied directly to the computational method used in this work. The CFD process implemented is time consuming due to the relatively high level of accuracy set for the solutions in the solver. The resources available also limit the selection of other comparable software, but due to the fact that university ESI Suite software was used a positive outcome in technology was realized. This outcome is related to the fact that the CFD lab at SJSU has six usable terminals to run simulations. Therefore, although the system was not clustered multiple runs can be run simultaneously on different machines to generate more data in the time required. Another added benefit is that different runs can be rerun as needed to improve the data and correct any mistakes. 


\section{Chapter 2 Computational Method Description}

The first step of solving the given problem involves addressing the geometry, flow conditions, and the requirements of the simulation in order to design a computational method. The order in which this thesis began to solve the specified problem is to identify the governing equations both for CFD and engine design, benchmark to verify the program, setup a compatible geometry, generate a mesh for computations, and finally address the boundary conditions desired. Once these things are accomplished then the full solution can begin to coalesce from the derived results.

\section{ESI Background}

ESI Group created the simulation software implemented in this study. The software available at SJSU consists of the ESI CFD Suite, which is essentially all of the main solvers created by ESI Group. This study made use of the CFD FASTRAN portion of the suite. The CFD FASTRAN software consists of a package of software components that need to be used in conjunction in order to generate a solution. The components of the software are as follows: CFD-GEOM, CFD-FASTRAN-GUI, CFDFASTRAN-SOLVER, and CFD-VIEW. CFD-GEOM is used to generation the geometry and the mesh required for the solver. It allows for either the importation of previously developed geometry or the creation of the software within its environment. The mesh can either be user generated or automatically generated as an unstructured grid. This 
study used a user made structured grid as it allows greater control and emphasis on key areas of the flow.

CFD-FASTRAN-GUI is a user friendly graphic user interface that allows the application of boundary conditions to a model that has been imported from CFD-GEOM. The model imported displays the grid and geometry generated in CFD-GEOM. The user can then setup the input parameters for the simulated run and launch the solution. At this point the whole setup and model can be saved for use again. CFD-FASTRAN-SOLVER can be viewed as the "back end" of the software that does all of the heavy lifting in terms of calculations and essentially solves the simulation. The results are then saved to either stop, start, or complete the simulation. CFD-VIEW allows the data generated from CFDFASTRAN-SOLVER to be displayed graphically. The data are displayed graphically for ease of interpretation and allows for the examination of the mesh and or solution results at different levels of depth. The data can then be saved and viewed later to compare with other runs.

\section{Governing Equations}

This study made use of two sets of governing equations to determine results of determining the contour of the nozzles. The first set will consist of the engineering calculations used to design a bi-propellant rocket found in the text by Huzel and Huang (1992). These calculations will create the foundation of the benchmark for the Titan I propulsion systems that will eventually be used in the second set of governing equations. The second set of governing equations will consist of the equations used by the different 
methods that can be selected in the CFD code used. This second set of equations determined the flow of the generated geometry and inputs determined from the first set of equations. Therefore, a description of each set was detailed in this work. 
Determining Titan I Engine Geometry. The Titan I engine geometry is not readily found in published literature; however, there does exist one very low quality image of what appears to be a contour with very faded numbers. This can be seen in the reference The Development of Propulsion Technology for US Space Launch Vehicles, 1926-1991 by J.M. Murphy. This is the origin of how the first 2D and 3D model was created in the popular computer aided design (CAD) tool known as CATIA by Dassault Systèmes.

Other images of the propulsion systems were found that showed a detailed isometric view, however these have no dimensions. Therefore, there use is limited to being used as a visual reference for understanding where the various components that make up the first and second stage propulsion units are found. They do display the different parts and components of the engines in clear detail and also provide detail as to how the stages were controlled. Figure 3 displays the first stage isometric view while the Murphy reference mentioned earlier displays a cutaway with a numbered style parts list the subsequent figures display information for the second stage propulsion system. 


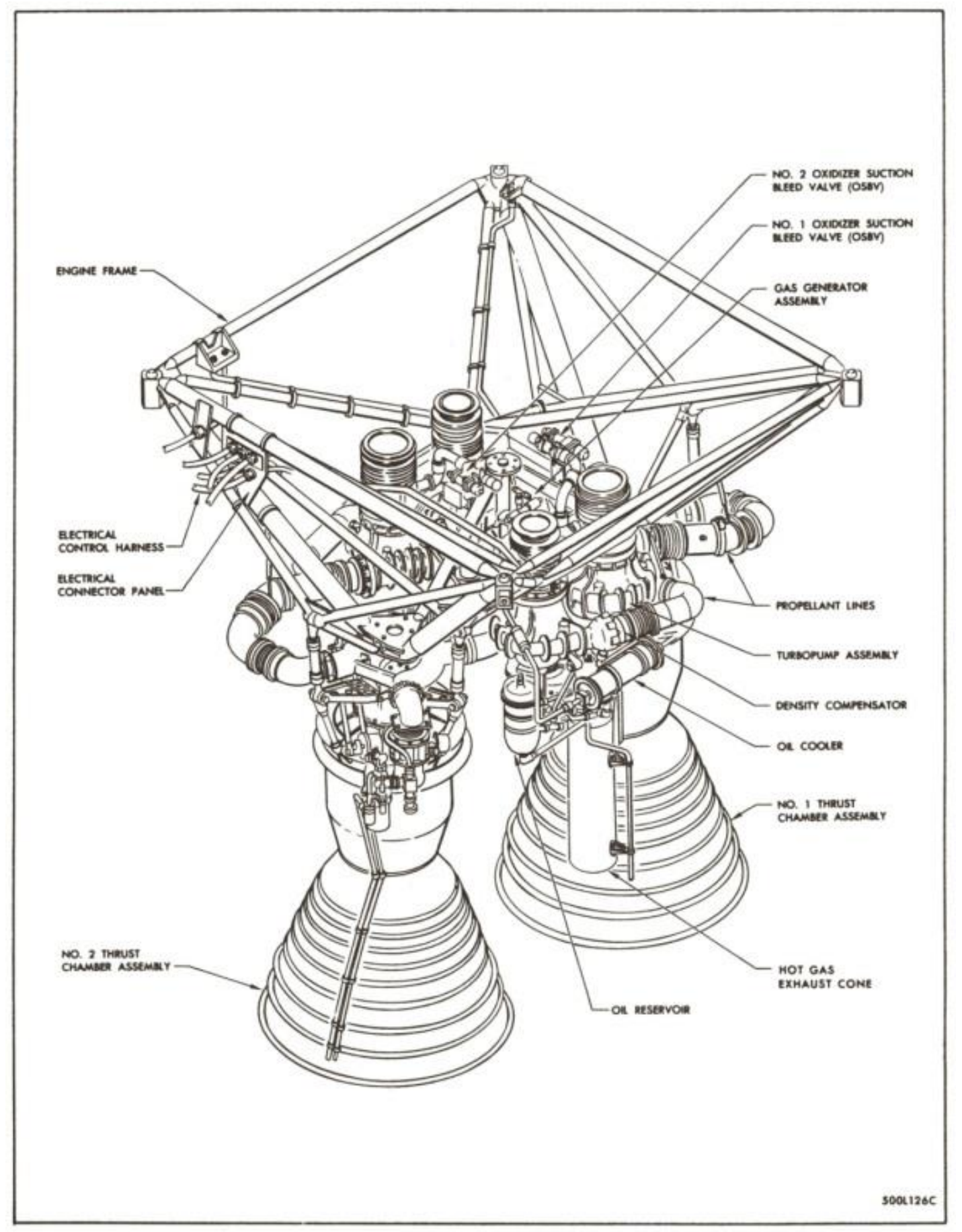

Figure 1-58. Stage I Engine

Figure 5 Stage I Isometric View 


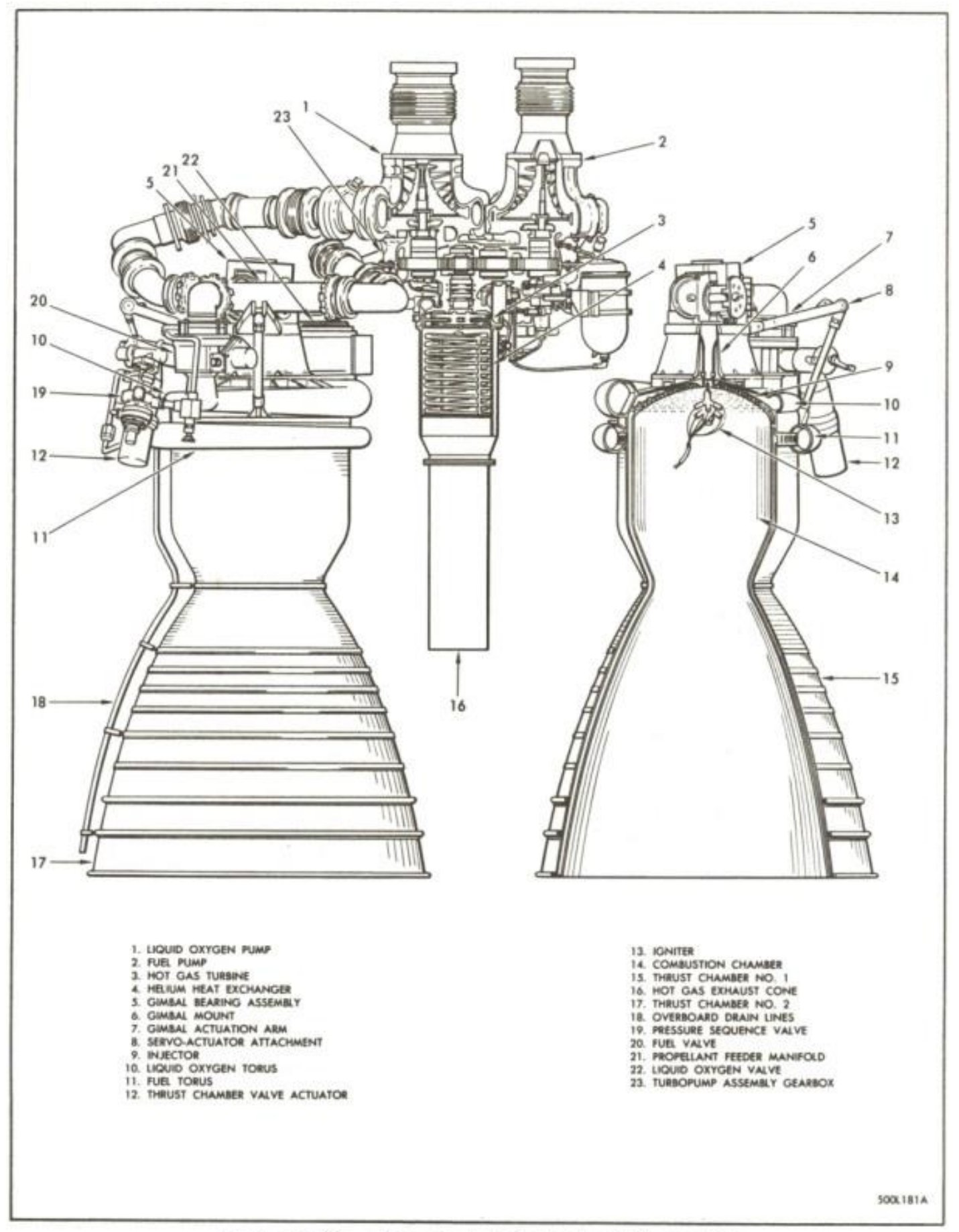

Figure 1-60. Stage I Rocket Engine Subassembly

Figure 6 Stage I Subassembly 


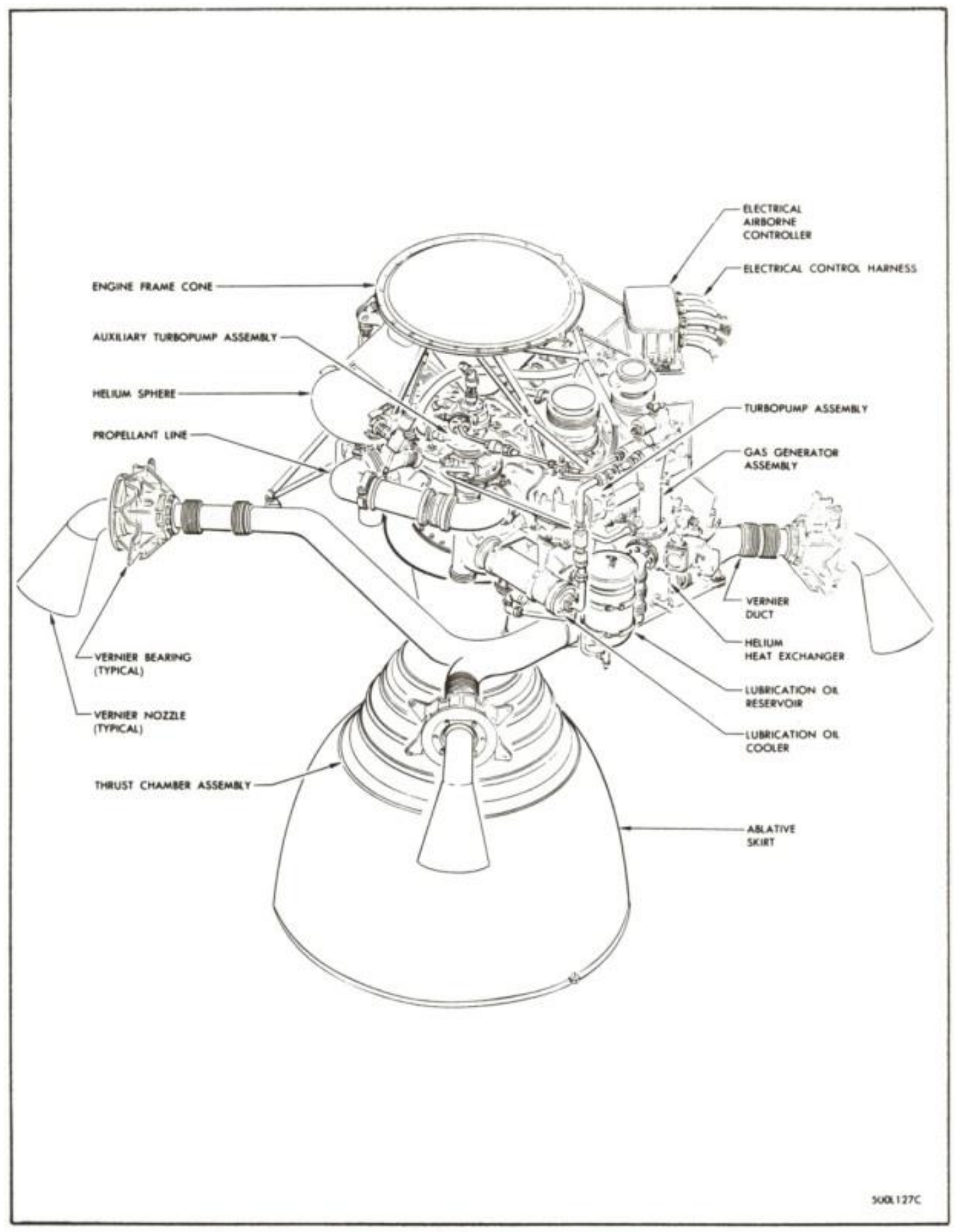

Figure 1-59. Stage II Engine

Figure 7 Stage II Isometric View 


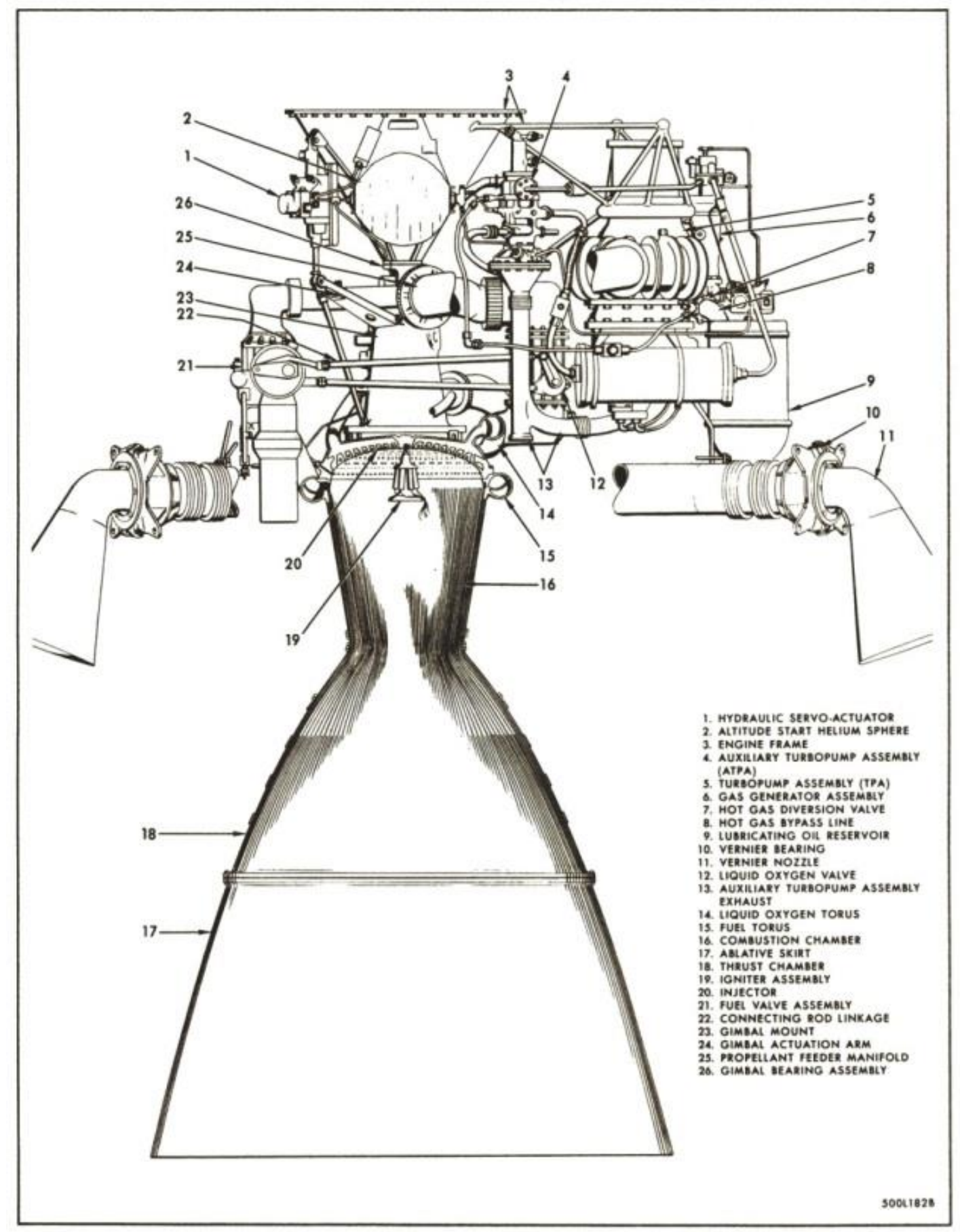

Figure 1-61. Stage II Rocket Engine Subassembly

Figure 8 Stage II Subassembly 
From the schematic in the Murphy reference mentioned earlier a CAD model was reverse engineered by importing the image directly into one of the planes in the CATIA program and tracing the outline of the engines. This was done because of the lack of literature containing the required dimensions of the Titan I propulsion system. The resulting 3D CAD models that were generated are displayed below in Figure 9.

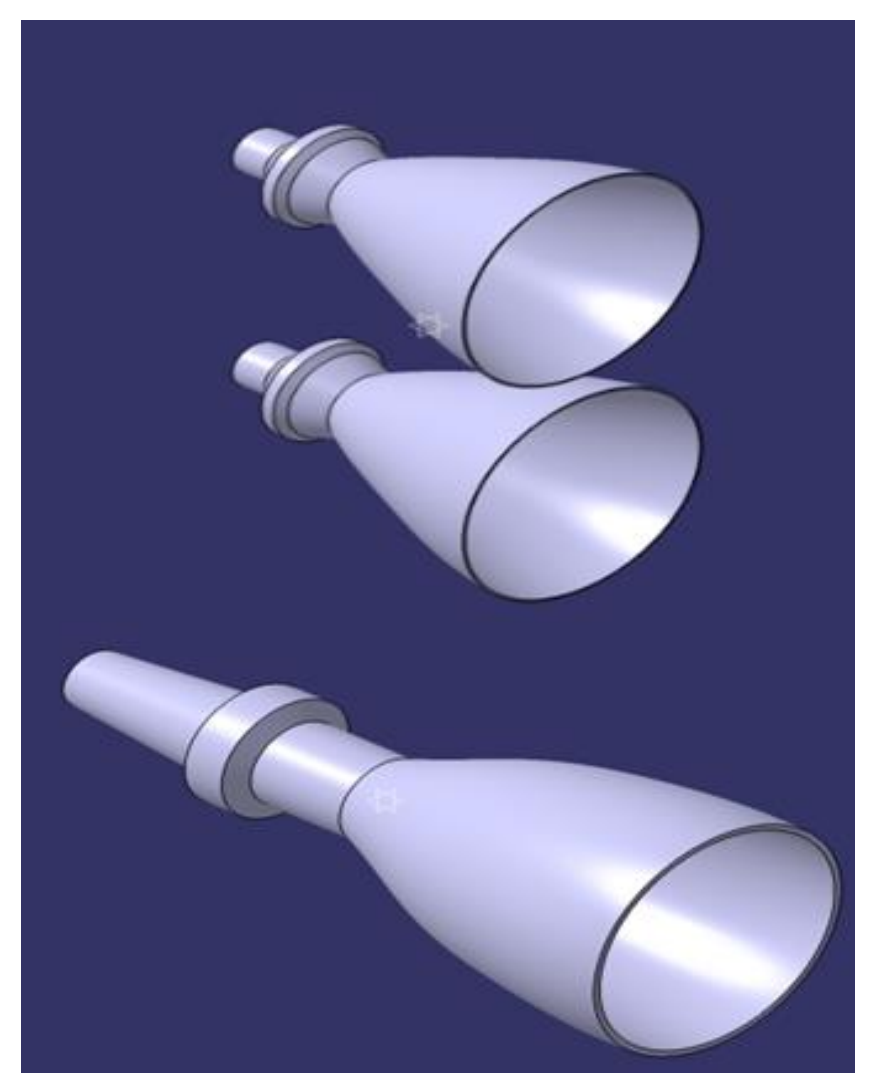

\section{Figure 9 3D CAD Models (Not to Scale) derived from Figure 2 Schematic}

The second set of geometry is generated from the published information about the Titan I propulsion systems displayed in Table 1 Stage I and II Engine Parameters above. From these values the equations for the various inputs and outputs were found and linked together in EXCEL in order to facilitate quick changes. The reason for the use of EXCEL is because a user can see the interdependencies of the equations through a 
selection of options, which allows a quicker method of checking the work. A portion of the EXCEL sheet is displayed in Figure 10 below and shows a portion of the initial sheet with input variables already inserted and highlighted in green. The yellow gold color grid boxes are outputs required for design of the geometry and the green color represents user inputs.

\section{ONLY ENTER THESE VALUES}

\begin{tabular}{|c|c|c|c|c|c|c|}
\hline \multicolumn{2}{|c|}{ Nozzle area expansion ratio: } & & Values & Units & Values & Units \\
\hline 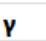 & \multicolumn{2}{|l|}{ Specific heat ratio } & 1.1485 & N/A & N/A & N/A \\
\hline Po & \multicolumn{2}{|l|}{ Chamber pressure } & 587 & psi & 4047223 & $\mathrm{~Pa}$ \\
\hline $\mathrm{Pe}$ & \multicolumn{2}{|l|}{ Exit pressure } & 14.7 & psi & 101353 & $\mathrm{~Pa}$ \\
\hline$\epsilon$ & \multicolumn{2}{|c|}{ Theoretical Nozzle area expansion ratio (AIAA p. 9) } & 6.53384 & & & \\
\hline \multicolumn{7}{|c|}{ Coefficient of thrust: } \\
\hline $\boldsymbol{v}$ & \multicolumn{2}{|l|}{ Specific heat ratio } & 1.1485 & & & \\
\hline $\mathrm{Pe}$ & \multicolumn{2}{|l|}{ Exit pressure } & 14.7 & psi & 101353 & $\mathrm{~Pa}$ \\
\hline Po & \multicolumn{2}{|l|}{ Chamber pressure } & 587 & psi & 4047223 & $\mathrm{~Pa}$ \\
\hline $\mathrm{Pa}$ & \multicolumn{2}{|l|}{ Ambient pressure } & 14.7 & psi & 101353 & $\mathrm{~Pa}$ \\
\hline$\epsilon$ & \multicolumn{2}{|c|}{ Nozzle area expansion ratio } & 6.53384 & & & \\
\hline Cf & \multicolumn{2}{|c|}{ Coefficient of thrust (AIAA p. 13) } & 1.54597 & & & \\
\hline \multicolumn{7}{|c|}{ Throat area: } \\
\hline Cf & \multicolumn{2}{|l|}{ Coefficient of thrust } & 1.54597 & & \multirow{2}{*}{\multicolumn{2}{|c|}{$667233 \mathrm{~N}$}} \\
\hline $\mathbf{F}$ & \begin{tabular}{l|l} 
Thrust & \\
\end{tabular} & & 150000 & lbf & & \\
\hline Po & \multicolumn{2}{|c|}{ Nozzle stagnation pressure } & 587 & psi & \multicolumn{2}{|c|}{$4047223 \mathrm{~Pa}$} \\
\hline At & \multicolumn{2}{|c|}{ Throat area (AIAA p. 13) } & 165.292 & in 2 & \multirow{2}{*}{\multicolumn{2}{|c|}{$\begin{aligned} & 1066.4 \mathrm{~cm}^{\wedge} 2 \\
& 368481 \mathrm{~cm}\end{aligned}$}} \\
\hline Dt & \multicolumn{2}{|c|}{ Throat diameter } & 14.5071 & in & & \\
\hline Rt & \multicolumn{2}{|l|}{ Throat radius } & 7.25356 & in & \multicolumn{2}{|c|}{$18.424 \mathrm{~cm}$} \\
\hline \multicolumn{7}{|c|}{ Exit diameter: } \\
\hline$\varepsilon$ & \multicolumn{2}{|c|}{ Contraction area ratio (AIAA p. 73) } & 8 & 3 & \multirow{2}{*}{\multicolumn{2}{|c|}{$368481 \mathrm{~cm}$}} \\
\hline Dt & \multirow{2}{*}{\multicolumn{2}{|c|}{$\begin{array}{l}\text { Throat diameter } \\
\text { Fxit diameter (AIAA } 82)\end{array}$}} & 14.5071 & in & & \\
\hline $\mathrm{De}$ & \multirow{2}{*}{\multicolumn{2}{|c|}{ Exit diameter (AIAA p. 82) }} & 41.0323 & 3 in & \multicolumn{2}{|c|}{$104.222 \mathrm{~cm}$} \\
\hline $\operatorname{Re}$ & & & 20.5162 & 2 in & \multirow{2}{*}{\multicolumn{2}{|c|}{$\begin{array}{c}52.111 \mathrm{~cm} \\
853118 \mathrm{~cm}^{\wedge} 2\end{array}$}} \\
\hline $\mathrm{Ae}$ & \multicolumn{2}{|c|}{ Exit radius } & 1322.34 & $\operatorname{in}^{\wedge} 2$ & & \\
\hline
\end{tabular}

Figure 10 EXCEL of Stage I Geometry Values

The calculator solves the following equations: specific impulse, characteristic velocity, thrust coefficient, throat diameter, exit diameter, chamber diameter, convergent cone length, nozzle length, mass flow rate, exit velocity, and others found in the text Modern Engineering for Design of Liquid-Propellant Rocket Engines. The final sets of 
values were compared to the published information and a percent difference was calculated. These differences were maintained below 10\%. The equations used as the major inputs for the EXCEL file that was created are listed below.

Theoretical nozzle area expansion ratio:

$$
\varepsilon=\frac{\left(\frac{2}{\gamma+1}\right)^{\frac{1}{\gamma-1}}\left[\frac{\left(p_{c}\right)_{n s}}{p_{e}}\right]^{\frac{1}{\gamma}}}{\sqrt{\frac{\gamma+1}{\gamma-1}\left[1-\left(\frac{p_{e}}{\left(p_{c}\right)_{n s}}\right)^{\frac{\gamma-1}{\gamma}}\right]}}
$$

Equation 1

Coefficient of thrust:

$$
C_{f}=\sqrt{\frac{2 \gamma^{2}}{\gamma-1}\left(\frac{2}{\gamma+1}\right)^{\frac{\gamma+1}{\gamma-1}}\left[1-\left(\frac{p_{e}}{\left(p_{c}\right)_{n s}}\right)^{\frac{\gamma-1}{\gamma}}\right]}+\frac{A_{e}}{A^{*}}\left[\frac{p_{e}-p_{a}}{\left(p_{c}\right)_{n s}}\right]
$$

Equation 2

Area of the throat:

$$
A_{t}=\frac{F}{\left(p_{c}\right)_{n s} C_{f}}
$$

Equation 3

Diameter of the exit:

$$
D_{e}=\sqrt{\varepsilon} \times D_{t}
$$


Length of the nozzle:

$$
L_{n}=\frac{R_{t}(\sqrt{\varepsilon}-1)+R(\sec \alpha-1)}{\tan \alpha}
$$

Equation 5

Characteristic velocity:

$$
c^{*}=\frac{\sqrt{g \gamma R\left(T_{c}\right)_{n s}}}{\gamma \sqrt{\left[\frac{2}{\gamma+1}\right]^{\frac{\gamma+1}{\gamma-1}}}}
$$

Equation 6

Weight flow rate:

$$
\dot{W}=A_{t}\left(p_{c}\right)_{n s} \sqrt{\frac{g \gamma\left[\frac{2}{\gamma+1}\right]^{\frac{\gamma+1}{\gamma-1}}}{R\left(T_{c}\right)_{n s}}}
$$

Equation 7

Specific impulse:

$$
I s p=\frac{F}{\dot{W}}
$$

Equation 8 
Velocity at the exit:

$$
V_{e}=\sqrt{\frac{2 g \gamma}{\gamma-1} R\left(T_{c}\right)_{n s}\left[1-\left(\frac{p_{e}}{\left(p_{c}\right)_{n s}}\right)^{\frac{\gamma-1}{\gamma}}\right]}
$$

Equation 9

Pressure at the throat:

$$
P_{t}=\left(p_{c}\right)_{n s}\left[\frac{2}{\gamma+1}\right]^{\frac{\gamma}{\gamma-1}}
$$

Equation 10

The method chosen to design part of the contour was the Rao Method because of the nature in which it idealizes most of the characteristics of the rocket chamber liner and still provides a $90 \%$ or greater efficiency (Huzel \& Huang, 1992). The Rao Method uses a set of ratios that essentially creates a parameterized nozzle design, which is displayed in Figure 11 below.

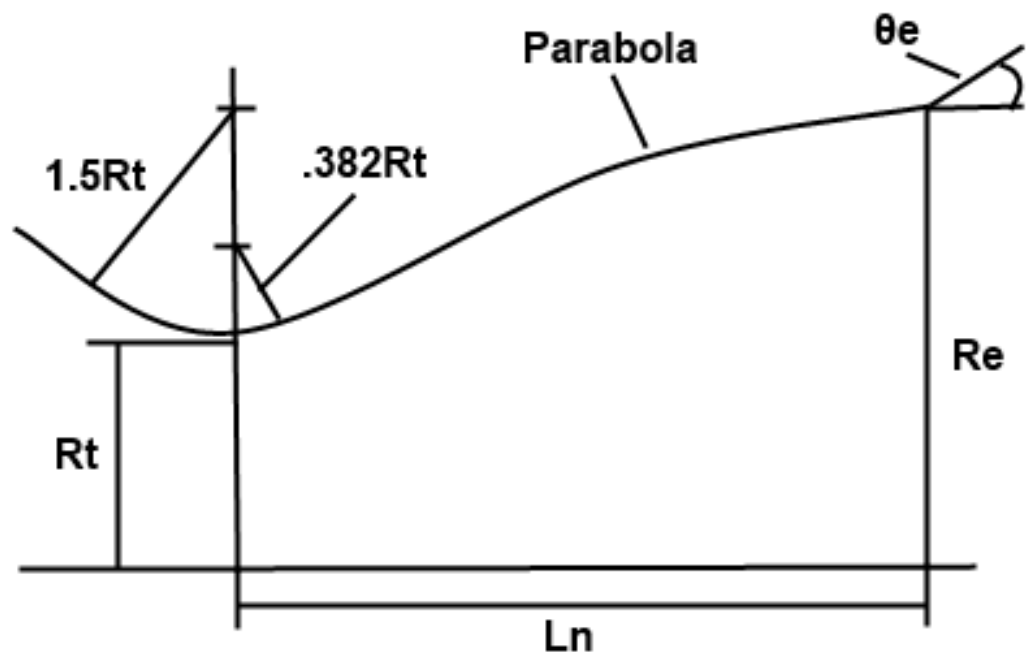

Figure 11 Parameterized Nozzle Design (Rao Method) 
What is clearly evident from this figure is the fact that the method clearly labels the main inputs of the design and largely helps to organize the efforts of the design into what needs to be solved first. The parameters required to fill out the template include the radius of the throat, length of the nozzle, the radius of the exit, the angle of the nozzle and the angle of the exit. The angles refer to the "initial" and "final" angles of the parabola be used to idealize the bell nozzle shape. This can be tricky to implement in a computer aided design $(\mathrm{CAD})$ program especially because the constraints in the program tend to want to "best fit" the parabola of the none-curved section. The figure below is generated from experimental data of actual rocket engines that have been developed. These angles are a sort of "best guess" through trial and error over the years of development for the bell nozzles. From this figure the initial and final angles of the parabola were used. 


\section{Computational Fluid Dynamics equations used in ESI FASTRAN}

SOLVER. The FASTRAN solver simulation methodology used a combination of models and approaches to develop an answer to the input problem with the set parameters. This allows the code to handle calorically perfect gases, mixtures of gases and/or moving bodies, inviscid, viscous or turbulent flows. There are four main methods and approaches used in solving the different flow options include Time-Marching, Conservative Density-Based Formulation, Finite Volume Discretization, and Upwind Approximations.

The Time-Marching approach is applied to either time-dependent or steady-state and will always have a set of initial conditions that is marched for a user specific set time. This approach allows the user to monitor residuals for steady-state solutions and can set an appropriate set of iterations until convergence is met.

The Conservative Density-Based Formulation method makes use of conservative laws using a density-based formulation. Essentially, this is very applicable to high-speed compressible flows. This method also works especially well when the flow being analyzed has shock waves, expansion waves, and other discontinuities.

The Finite Volume Discretization method discretizes and numerically integrates the governing equations based on a finite-volume approach. It allows the flow domain to be divided into discrete points where control volumes and be constructed. This method allows for the use of either unstructured or structured 
grids and the information of the flow is stored in the center of the cell. The advantage of this method over others is that it is internally conservative and the governing equations do not require any transformation before being implemented. Essentially, this method allows very large and difficult problems to be solved more easily than if a finite-element discretization was to be employed. Therefore, larger more detailed grids can be input into this method allowing for more accurate results.

The Upwind Approximations implemented by FASTRAN currently are the Roe's flux difference splitting and Van Leer's flux vector splitting schemes. What the upwind approximation methods do is connect the flow from one cell to the next by evaluating the flux across the common face using only the information from the upwind direction. Therefore, for supersonic flows in which information propagates only in one direction this method of solution is preferred for modeling a flow pattern.

The governing equations ultimately used by the FASTRAN CFD code are dependent on the problem being solved. In this case the flow field temperatures for the applied problem will require the conserving of mixture fractions or mass of each of the chemical species. Furthermore, due to the fact that the flow speed is fast and relatively low density additional energy equations will need to be solved in the complete set of partial differential equations. The governing equations will also be determined based on whether the flow is viscous and/or turbulent. However, because the flow being analyzed has been determined to be a 
viscous flow the set of equations used becomes the Navier-Stokes equations for laminar flows. Additional terms are added to the flow due to the inclusion of turbulence which includes terms for momentum and energy.

Finally, the assumption that the flow is based on a continuum model affects the flow field equations. The Navier-Stokes Equations describe the motion of a viscous, heat conducting, compressible fluid (Liepmann, 1957). These equations provide a conventional mathematical model of a gas as a continuum. Within these equations the macroscopic properties are dependent variables, while the independent variables are the spatial coordinates and time (Bird, 1994). Generally, according to Bird, the traditional requirement for using the Navier-Stokes equations is that the Knudsen number should be less than 0.1 . The Knudsen number is a dimensionless number, which is defined as the ratio of the molecular mean free path to a specific representative physical length scale. The length that is used could be the radius of the body in a fluid. The equation to determine the value of the Knudsen number is shown in Equation 11. Essentially, the Knudsen numbers help to determine whether based on the problem being analyzed the Navier-Stokes equations are valid to use and in this case they are due to the continuum model that was applied.

Knudsen Number:

$$
(K n)=\frac{\lambda}{L}
$$


$\mathrm{L}$ as the length of the macroscopic gradients:

$$
L=\frac{\rho}{\left(\frac{d \rho}{d x}\right)}
$$

Equation 12

The Navier-Stokes set of equations is composed of three equations. The primary equation is the momentum equation, which is a vector equation that has had Newton's Law of Motion applied to a fluid element. The other equations of continuity, also known as mass conservation and energy make up the other set of equations that supplement the momentum equation to form the Navier-Stokes equations. All of these equations are displayed below. Furthermore, this study will take advantage through the use of FASTRAN code the possible use of simplified governing equations which include the potential-flow equations, the Euler equations, and the thin-layer Navier-Stokes equations. The boundary conditions selected will also have an effect on, which version of the equations was used in order to speed the process of solving the problem. This was taken care of mostly through the code use, but user input will still be needed to guide the solutions.

The equations shown below describe the flow field conservation equations mentioned earlier. These equations need to be taken into account because a nonmoving control volume is being analyzed. Equation 13 is the general continuity equation used. Rho is the mixture density and $\mathrm{u}_{\mathrm{i}}$ is the mass averaged velocity in 
the $\mathrm{x}_{\mathrm{i}}$ direction. This is the only mass conservation equation that is required for a calorically perfect gas.

Continuity Equation:

$$
\frac{\partial \rho}{\partial t}+\frac{\partial \rho u_{i}}{\partial x_{i}}=0
$$

Equation 13

The conservation of momentum is shown in Equation 14. $\mathrm{P}$ is the pressure, $\mathrm{k}$ is the turbulent kinetic energy, $\mathrm{d}_{\mathrm{ij}}$ is the Kronecker delta, and $\mathrm{t}_{\mathrm{ij}}$ is the shear stress tensor. The right hand side is removed when analyzing inviscid flows and the $\mathrm{k}$ term disappears for laminar viscous flows or algebraic turbulence models. This form is valid for multi-species and multi-mixture gases as well as calorically perfect gases.

Momentum:

$$
\frac{\partial \rho u_{i}}{\partial t}+\frac{\partial \rho u_{i} u_{j}}{\partial x_{j}}=-\frac{\partial}{\partial x_{i}}\left[-\left(p+\frac{2}{3} \rho k\right) \delta_{i j}+\tau_{i j}\right]
$$

Equation 14

The conservation of mass expression displayed in Equation 15 is changed due to the fact that mixing and chemical equations have been enabled. The mixture density for chemistry cases such as the one being studied is obtained by the summation of the densities of the species. Where $E_{\text {int }}$ is the molecular international energy per volume, $\mathrm{e}_{\mathrm{int}, \mathrm{s}}$ is the molecular energy per mass for the species " $s$," qint,j is the heat flux of the internal energy in the $\mathrm{j}^{\text {th }}$ direction, and $\omega_{\text {int }}$ 
is the source term associated with the potential difference between the internal energy and the equilibrium energy of the mixture. The internal energy equation is based on the assumption that all the molecular species can be represented by one internal temperature (Cfd-fastran overview, 2010). This assumption decreases the time required for solution.

Mass:

$$
\frac{\partial E_{\text {int }}}{\partial t}+\frac{\partial}{\partial x_{j}}\left(E_{\mathrm{int}} u_{j}\right)=\frac{\partial}{\partial x_{j}}\left(\sum_{s} e_{\mathrm{int}}, s^{J} s, j-q_{\mathrm{int}}, j\right)+\omega_{\mathrm{int}}
$$

Equation 15

\section{Geometry and CAD Generation}

The geometry that was used in this study was both in the form of a 2D

axisymmetric and 3D representation of the propulsion systems presented. The geometry and subsequent $\mathrm{CAD}$ generation was devised from the most original version of the Titan I propulsion system schematics and published data. This study used the CAD program known as CATIA. The reason for using this software was because of the previous familiarity and experience of the user with the software and because of the different software options the program provides. The schematics used for the generation of the first set of models were found in an IAF text (Murphy, 1976). The CAD models were designed from this schematic using a tool in CATIA called "paint gallery," which allows the importation of 2D figures directly into the planes of operation. Some preparation is required before the image is imported into the program. First, the original schematic was 
edited to display only the areas of interest this was done in order to reduce unnecessary white space in the drawing.

Once these images were edited they were imported into CATIA using the tool described and the only requirement for this operation to function was the selection of the "work plane." This step is imaged below in Figure 12. In the center of the image there is a prominent red square. This square denotes an exaggerated highlighted plane where the image now coexists.

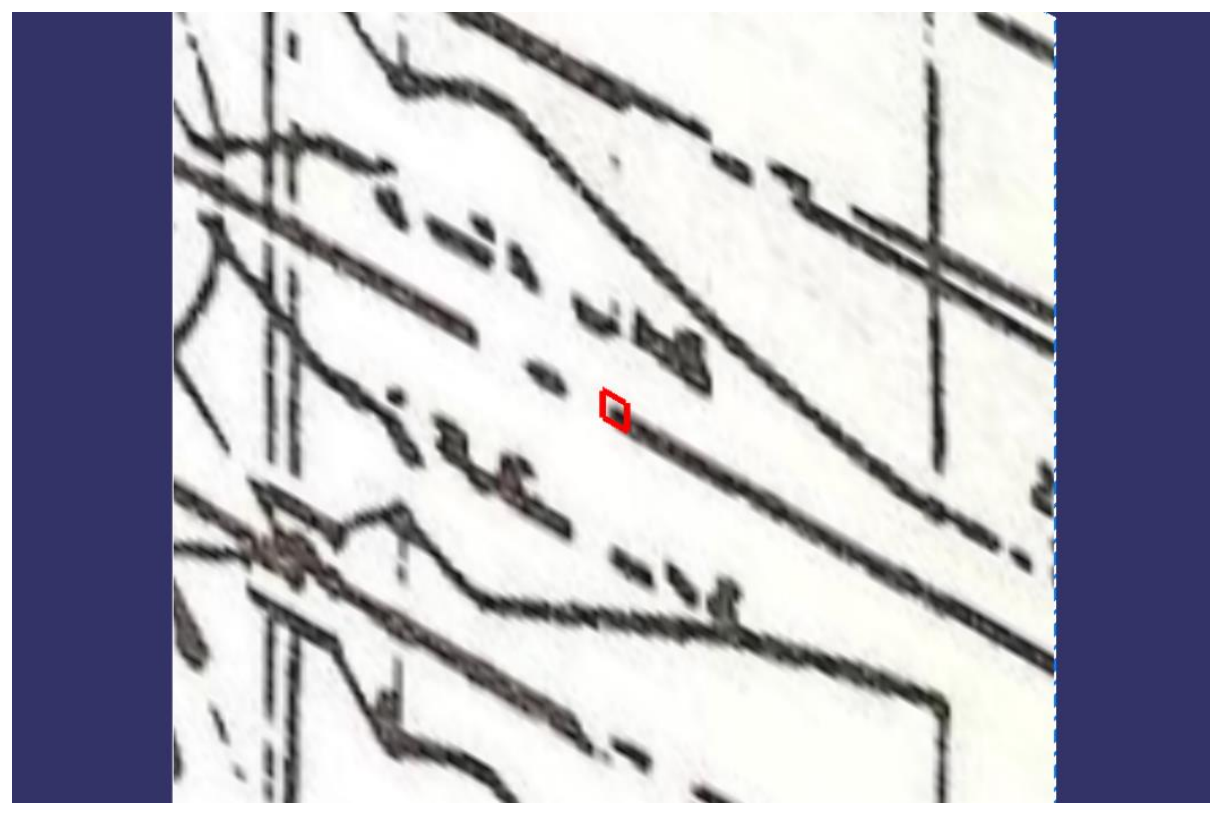

Figure 12 Close-up of image in work plane

Essentially, a user is able to trace the dimensions from any schematic into the program and from there be able to apply it in a more 3D friendly environment for visual and practical purposes. Figure 13 Outline of CAD Model on Schematic exhibits the display a user sees when performing the tracing actions. The tracing is as precise as the 
user's inputs when creating the upper outline that will eventually be rotated about a central axis due to symmetry. This study created some sample points on the original curve in order to better form the line with a modeling tool to create lines called the "spline" function. Normally, this function is used to generate curves from one point to the next, however if a user places points and then traces them with the "spline" function a more natural curve is generated. The shape of the curve is controlled with the placement of the last point on the model. This method was used exclusively for the nozzle. The standard method of using the "profile" tool to create lines of varying lengths was implemented to create the other portions of the shape.

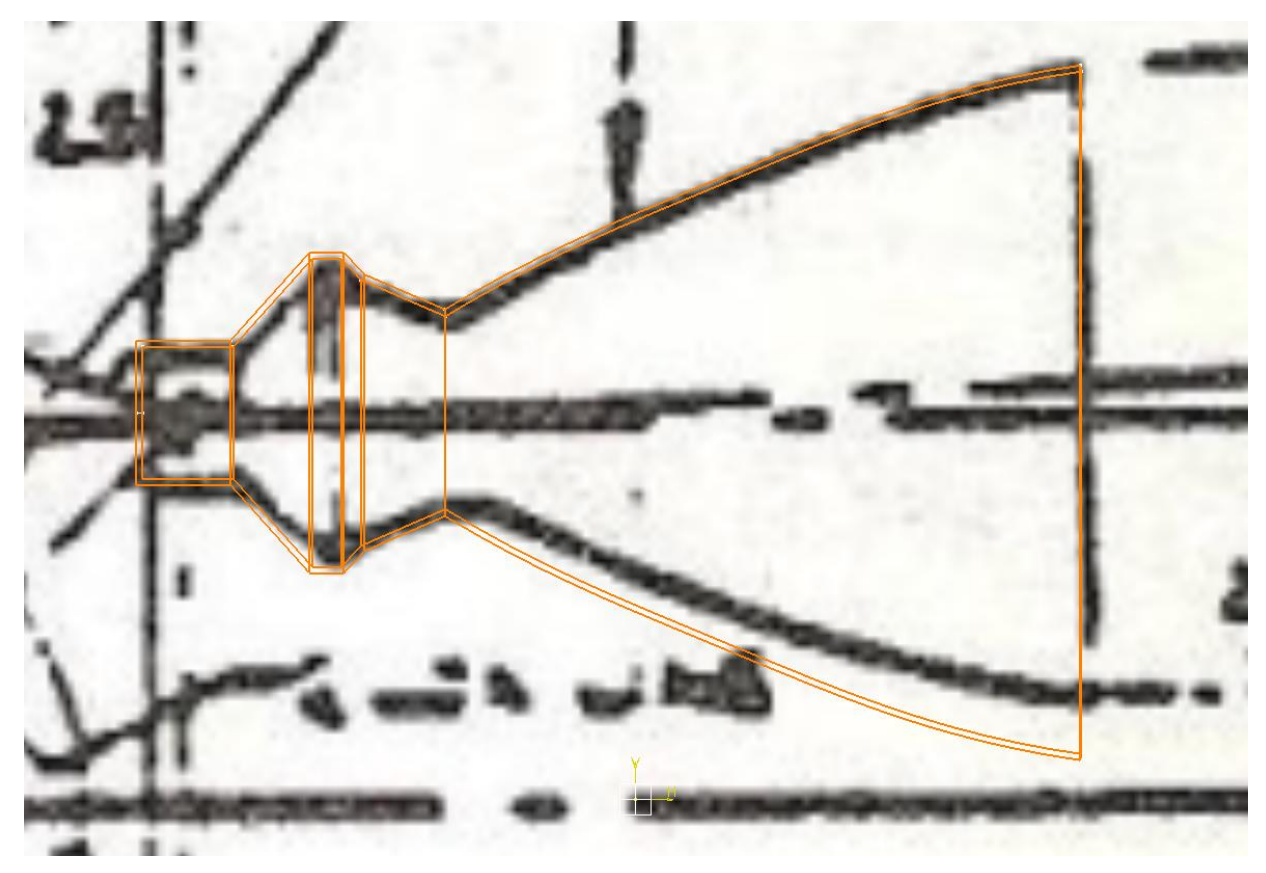

Figure 13 Outline of CAD Model on Schematic

Figure 13 Outline of CAD Model on Schematic displays the outline of the CAD model in orange for contrast on the original schematic. Essentially, a user can take any 
sized schematic and modify the scale to create a true 1:1 model of the image. This ability to create a 1:1 model is done through the implementation of one major dimension such as length, which generally drives the generation of any design. The dimensions if known on the original schematic can then be applied to the model in order to understand and then be gridded for solution. Once the final outline is created it can be "shafted," which means in CATIA terms revolving the outline about a central axis that user inputs and then selects. When this is correctly done the final result is displayed in Figure 14 below. The original image that was inserted as a guide can then be deleted and or hidden depending on the user's choice.

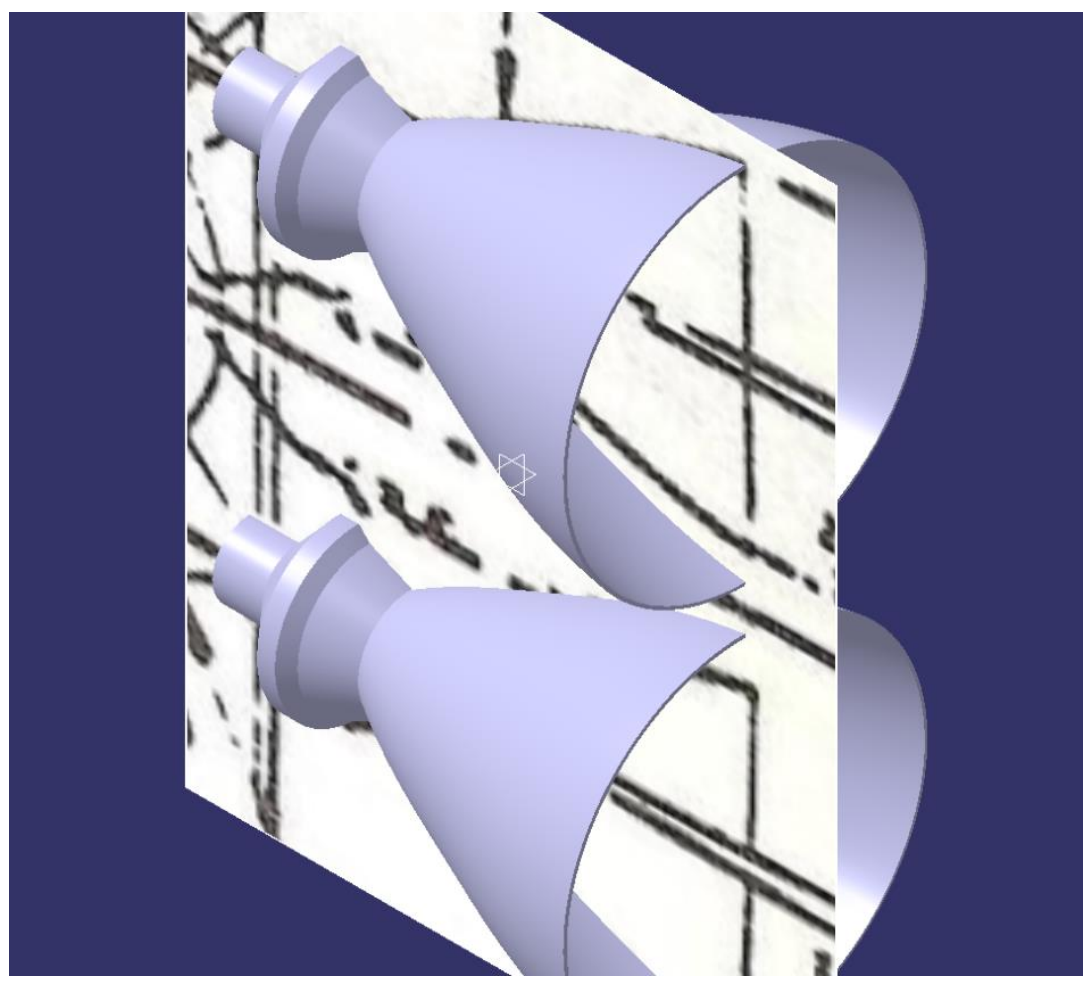

Figure 14 Final step in the model creation procedure 
The second set of geometry was developed through the use of the inputs found in Table 1 Stage I and II Engine Parameters. Following the procedure of creating an EXCEL calculator and resolving other values from the earlier sections a final set of values was created. These final values were input into the Rao Method template found in Figure 11 Parameterized Nozzle Design (Rao Method). This template was created in CATIA and can be seen in Figure 15.

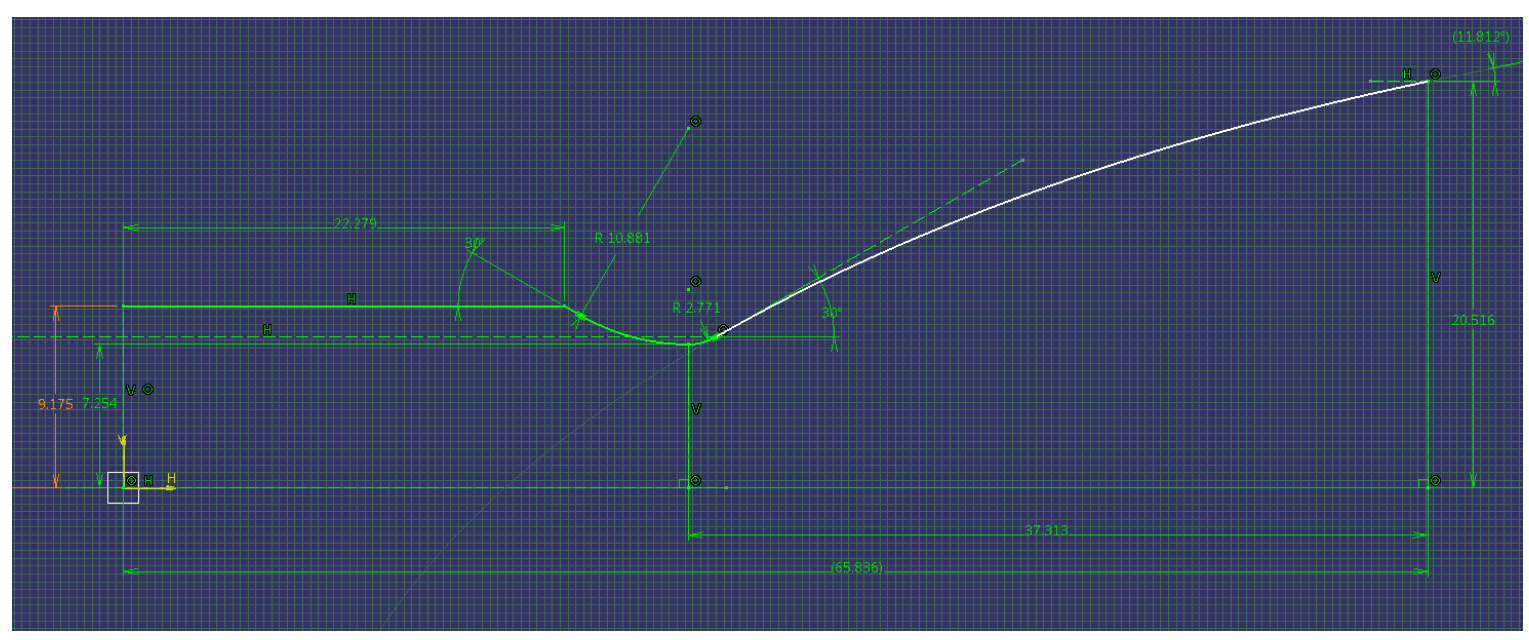

Figure 15 Rao Method Template for Stage 1

Essentially, the 2D and 3D models were able to be systematically generated with this method and thus the results of this can be seen below in the following figures. Figure 16 displays the first stage engine by itself and Figure 17 displays the second stage engine. Both models have an arbitrarily small thickness to give the model shape for visual purposes. It is important to note that these models are as accurate as possible based on the output parameters of these engines. These calculated models was the ones that was implemented into the FASTRAN CFD code in order to calculate the sought after flow solutions of the original Titan I propulsion system setup. 


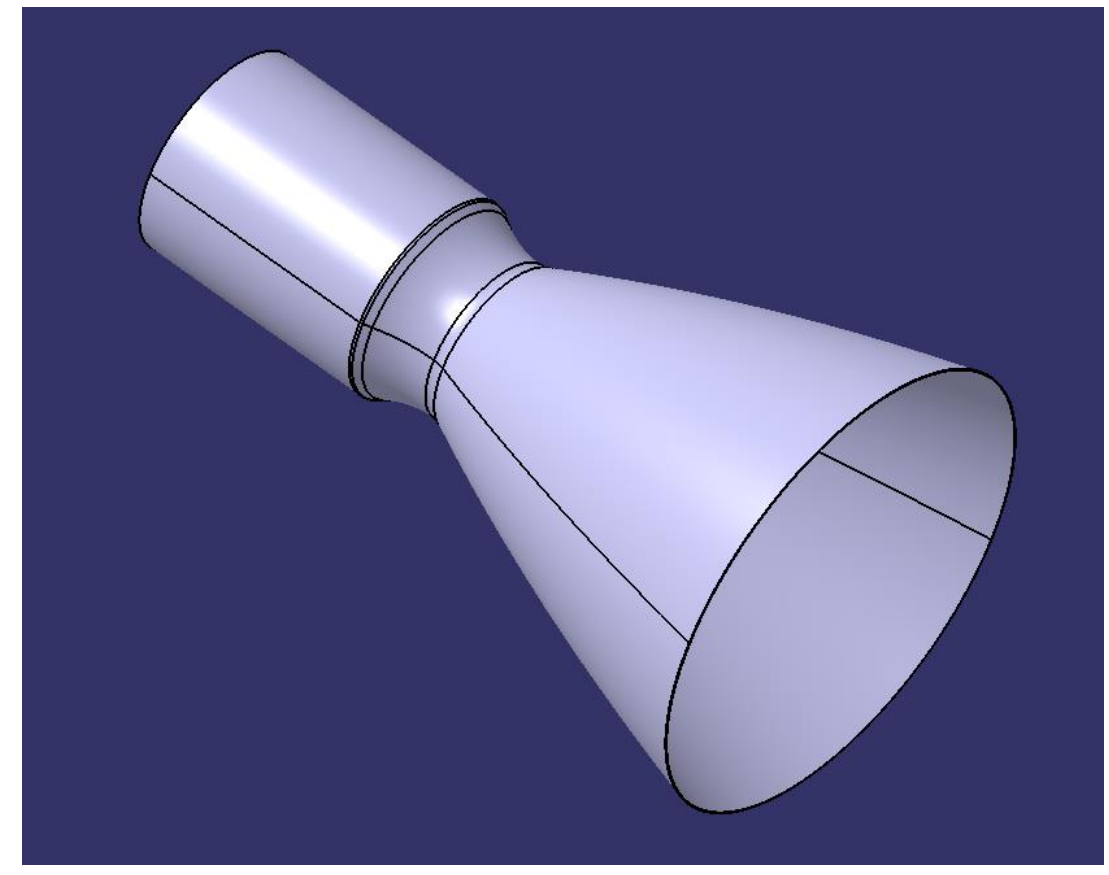

Figure 16 Stage I CAD Model from Geometric Calculations

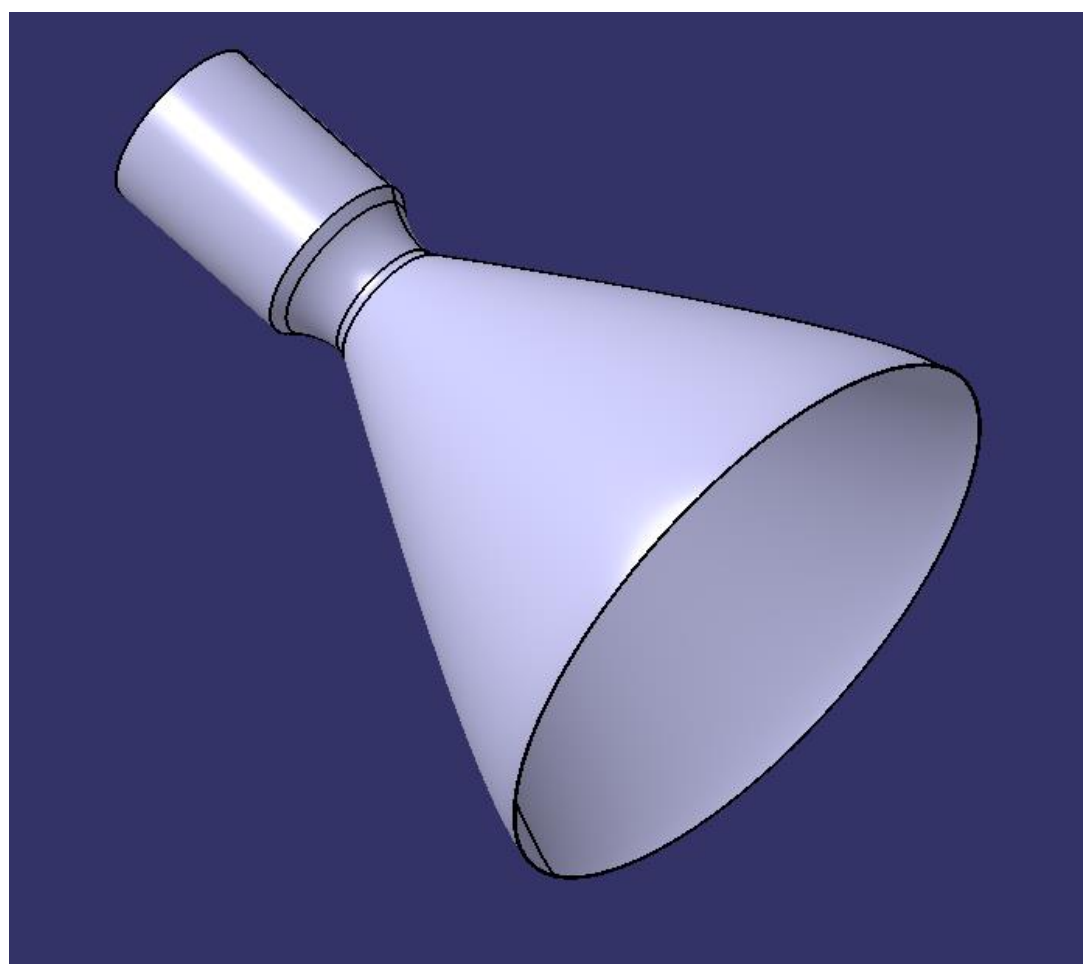

Figure 17 Stage I CAD Model from Geometric Calculations 


\section{Grid Setup}

The Titan I propulsion systems considered in this study was modeled in grid form in $2 \mathrm{D}$ axisymmetric domains. The software used for grid generation in this thesis was the built in grid program found in FASTRAN. The built in grid generation module allows for the user to create structured and or unstructured grids. The built in program accepts any arbitrary structure or unstructured grid generation by an external program. The structured grid generation program is based on transfinite interpolation (TFI) methodology (Cfd-fastran overview). This program supports uniform, exponential, geometric, and hyperbolic tangent grid point distribution along the edges. This means that the user can create a variety of concentrations of cells in order to capture the appropriate boundary layer interactions with the fixed geometries input. The user is also able to quickly determine how many cells will make up the grid by manually setting the points that will anchor the grid around the geometry. The creation of the grid is governed primarily by the topology that is input into the program.

The first step to create a run in ESI FASTRAN is setting up a grid in CFDGEOM. The grid setup follows four main steps: (1) first the geometry is created or imported; (2) then, a structured mesh is generated through user input and selection of options; (3) after that, boundary and/or volume conditions are set (in this case, only the boundary conditions are set); (4) finally, the entire setup is saved in a .DTF format to make the work compatible with the FASTRAN solver. 
CFD-GEOM allows users to hand-generate contours (or other shapes) with the built-in tools of the software. The program uses a streamlined method of generating both grid and geometry, making the process less complicated. The internal method uses a coordinate system, points, lines, and curves as the main components of geometry generation. However, the user is also permitted to import user-generated CAD models into the program. Various formats are supported, and in this study, the format IGES was used in Solidworks. For this study, the geometry was created outside of CFD-GEOM in Solidworks in order to expedite geometry generation. Figure 18 displays the imported view of the geometry from Solidworks. The figure displays only half of the generated contour. This is because the solver will make use of the fact that the geometry is symmetric about the $\mathrm{x}$-axis. This symmetry allows for reducing the complexity of the grid thus enabling faster results.

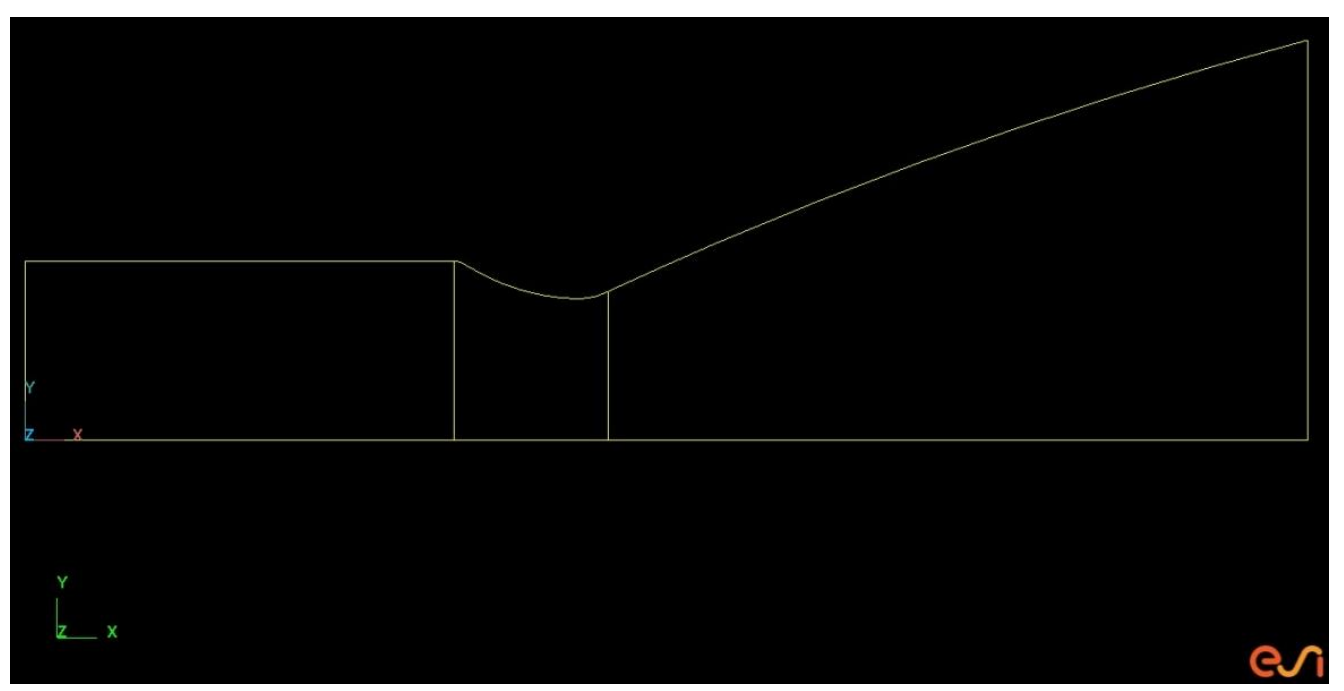

Figure 18 Imported View of the Geometry from Solidworks 
This program is capable of generating and supporting both 2D and $3 \mathrm{D}$ topologies and grids, and can be automatically updated to enforce the orthogonality by enabling smoothing algorithms. Next, Figure 19 displays a close-up of the structured grid that has undergone the process of smoothing to enforce orthogonality near the boundary layer. This figure is only a section of the larger implemented grid, and how this was made was discussed later. For this study, the focus has been on 2D topologies due to the nature of the symmetric shape being studied. Grids can be generated automatically or under userdefined parameters. Automatic grids come in either tetrahedral, pyramid, or prismatic meshes. However, a user-defined grid was used to adequately account for the boundary layer of the internal contour.

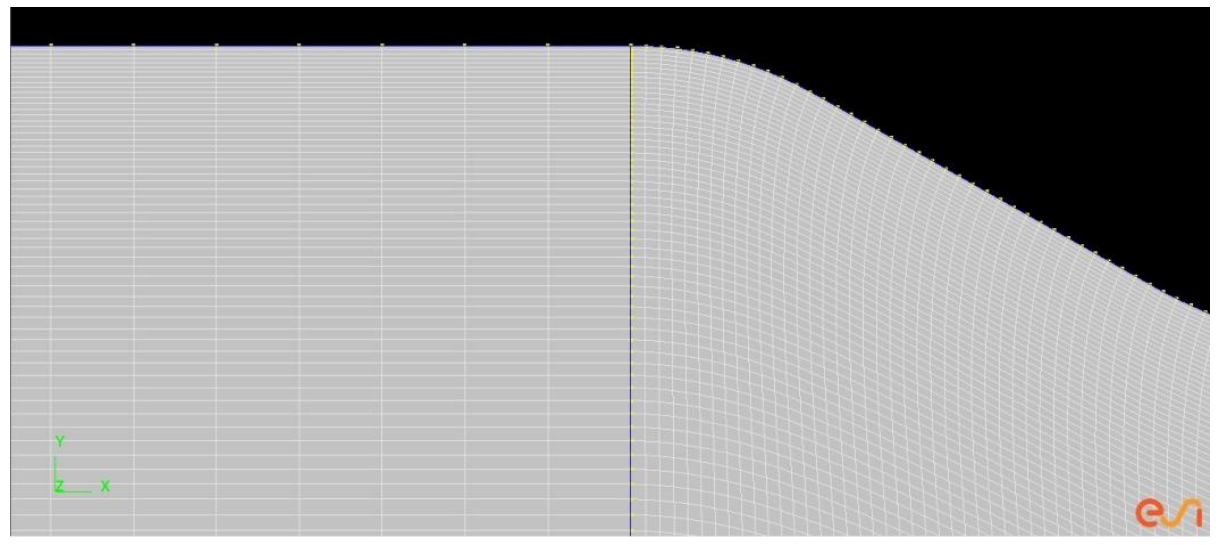

Figure 19 Close-up of Boundary Layer of Stage One Grid

User-generated grids are created from opposing edges. Procedurally, grids in the structured method use a "bottoms up" approach and are applied to the edge, the face, and finally a "grid" block is generated. The edge is the basic element for surface grid generation. An edge is an element connected in sequence with another, using points as 
the contact reference. These edges have at least one line segment connecting grid points, typically following the $\mathrm{n}$ grid points and $\mathrm{n}-1$ function for the line or edge element.

Figure 20 displays the edges of the imported geometry with grid points that were selected by the user. The image represents one step prior to grid generation.

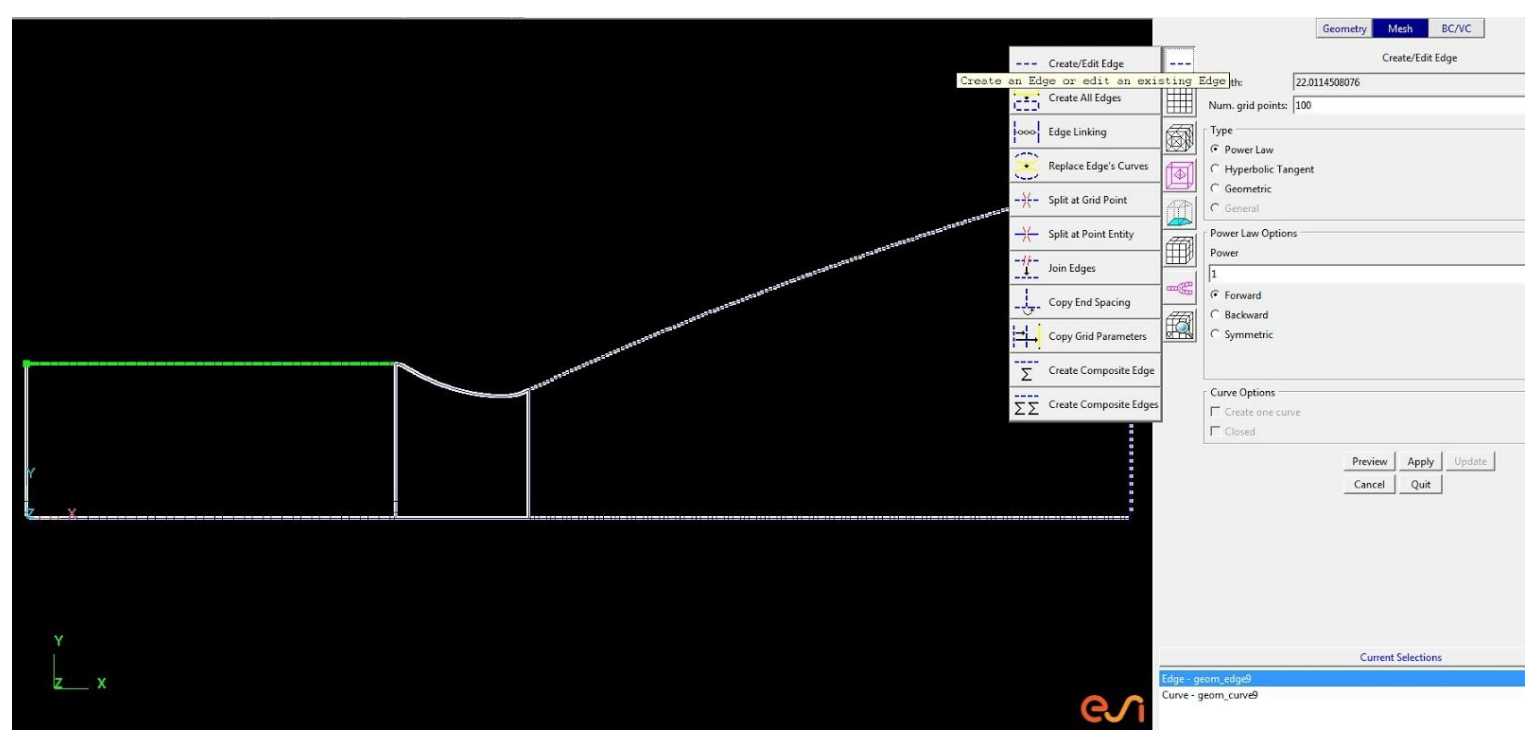

Figure 20 Imported Geometry Edge with Grid Points

Once all edges have been made the grid can start to be generated. Figure 21 displays the application process of the grid to nozzle: each of the edges have been selected along with the points to generate the grids, which can then be referred to as "surface faces." The surface face is a set of four edges that form the basic construction of the grid. These can be created by the user as needed and usually look like zones of grids on the geometry. 


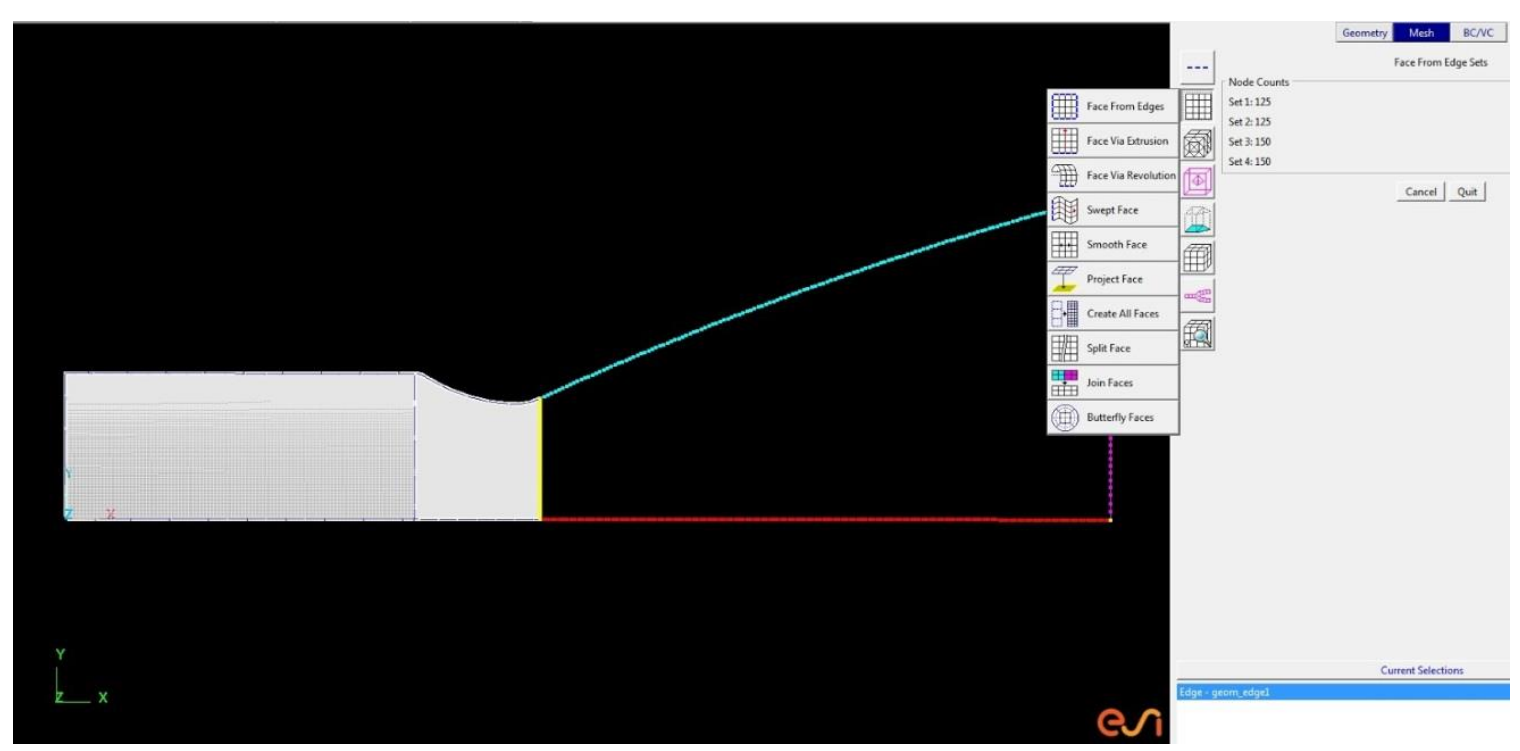

Figure 21 Grid Application to Nozzle Zone

Figure 19 above displays a portion of the grid and a close-up of the "boundary layer," cells near the contour wall. This is chosen by user manipulation of many grid points "bunched up" near the outer edge. This method of bunching points near the edge needs to be carried through in the same fashion throughout the zones in order to reduce errors during the solution process. Figure 22 displays the resulting complete grid after all the settings and smoothing algorithms have been applied by the user. From this point, the grids are joined into 2D Blocks. 


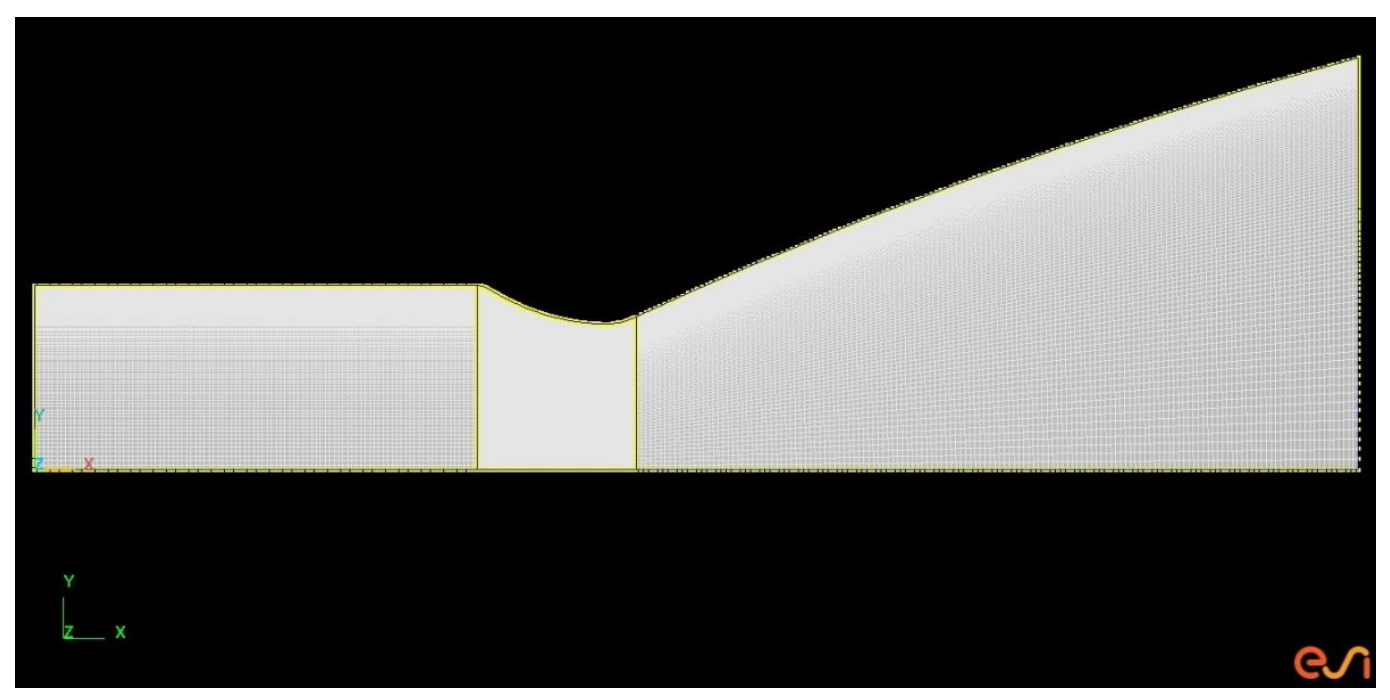

Figure 22 Whole View of Boundary Layer of Stage 1

2D Block generation is how grid zones are joined together to form a recognizable unit. A solver will use this $2 \mathrm{D}$ block to apply the necessary equations and initial conditions to the problem to generate a solution. Thus, it is critical to keep each zone uniform with the next in order to reduce opportunities for error. Figure 23 displays the $2 \mathrm{D}$ blocks being generated. The program changes the color of the zones from clear white to purple in order to indicate to the user that the block has been generated. 


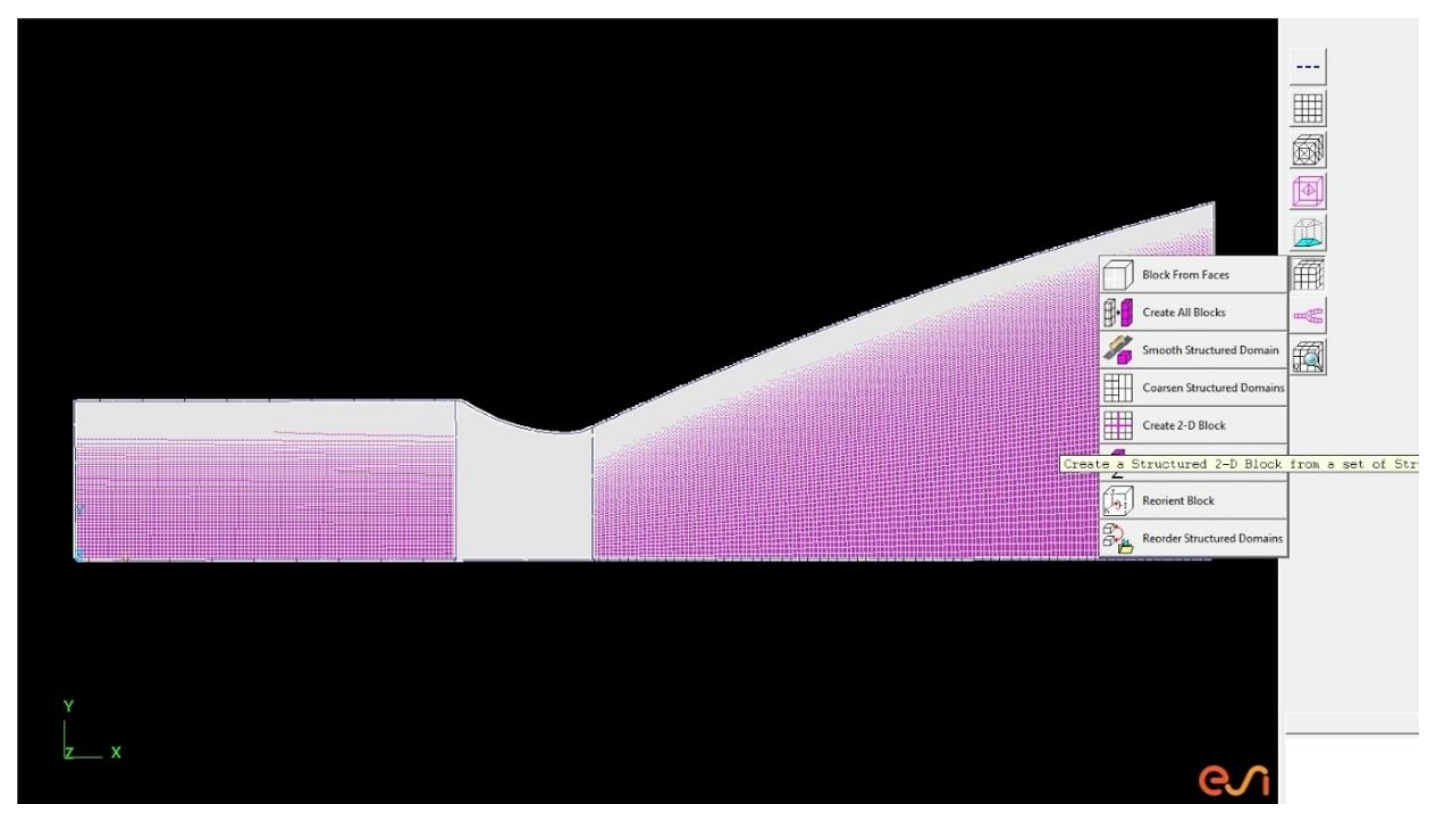

Figure 23 2D Block Generation on Grids

Finally, once the grid blocks have been created, boundary conditions must be set. In CFD-GEOM, this means that the boundaries need to be defined. The most important things for the user to specify are: (1) the wall, (2) the line of symmetry, and (3) the inlet and outlet. This step is crucial in order for the program to identify which zone to be treated in which particular way. Correct application of the line symmetry is also important, since it is an option in the solver. If the line of symmetry is not selected in the model, solver errors and/or an incorrect flow field was occur. Figure 24 displays how each "key" or edge location is chosen and its type options viewable through the drop down menu. Once this is done, the model can be saved in the .DTF format which allows the problem to commence solution. 


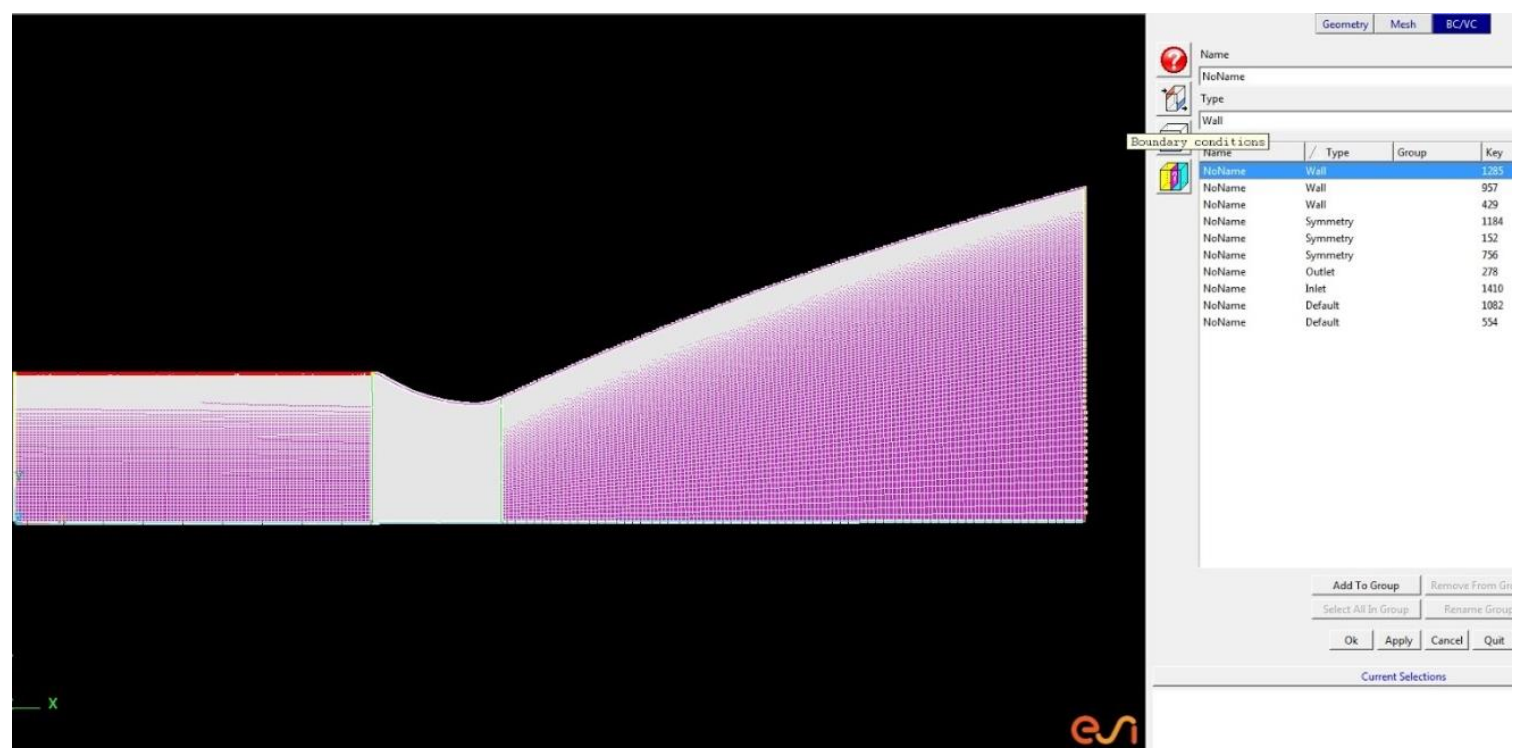

Figure 24 Selecting Boundary Condition Section Types

The final setup of the grid consists of 56250 cells for both grids, stage one and two. These cells are divided in three sections: section one, the chamber portion, is composed of 125 by 100 cells; the second throat section is composed of 125 by 200 cells; finally, the third nozzle section is composed of 125 by 150 cells. Each section contains evenly spaced cells along the $\mathrm{x}$ axis, but along the $\mathrm{y}$ axis, the cells are intentionally "bunched" closer to the upper edge of the geometry by a transition factor of 1.025 forward. The throat section has been smoothed orthogonally via 300 iterations; this is how the curve of the cells is able to continue the boundary layer shape selected by the user. Figure 25 aids in displaying the location of the cell amount locations. 


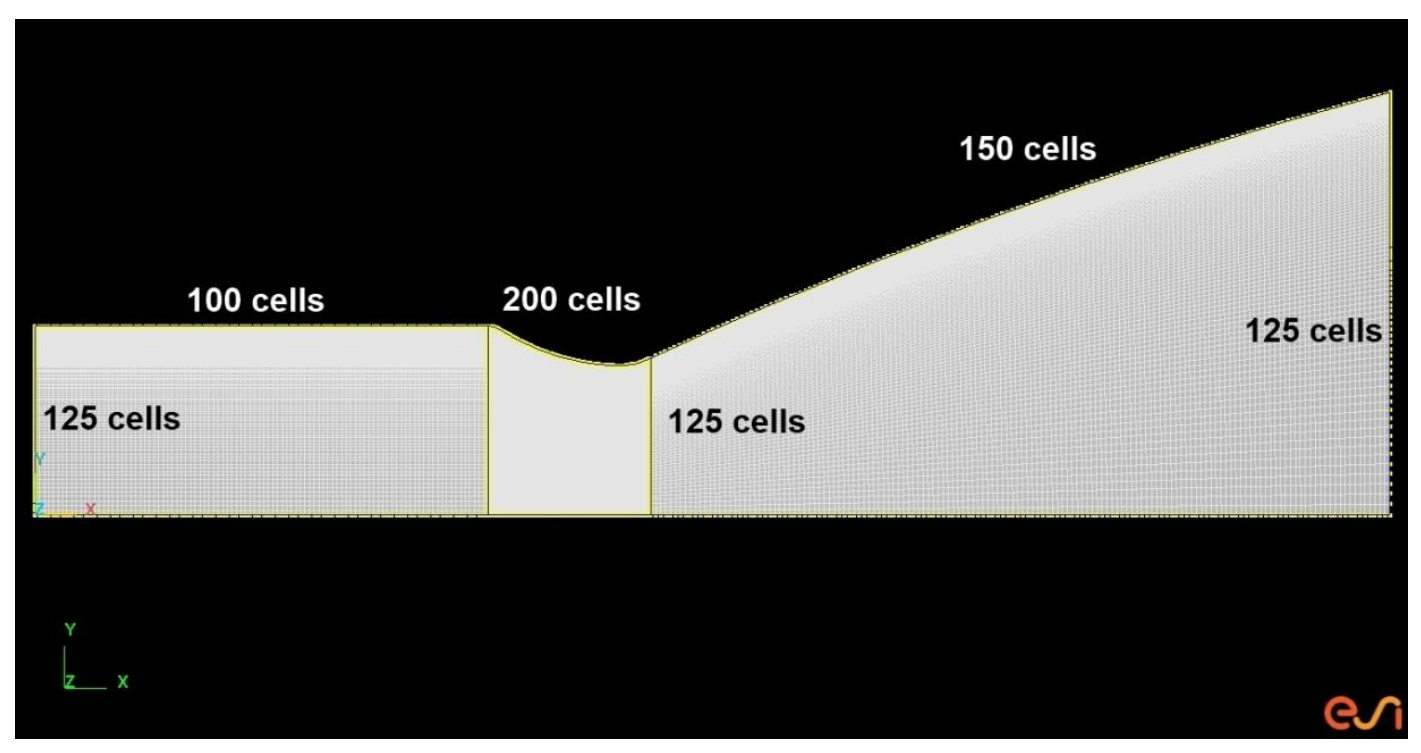

Figure 25 Number of Cells by Zone

\section{Solver Setup}

Once the grid has been finalized in CFD-GEOM, it is imported into the solver.

Once the model is imported, the user is presented with a graphical representation of the grid geometry and then given the choice of solution modules via tabs. The different modules have various purposes, but two of particular interest to this study is: (1) compressible flow, and (2) reacting/mixing fluids. The first option allows the user to study the flow going through or over geometry and allows for the input of flow-related parameters. The second option allows the user to choose variables that APPLY the fluid's chemistry TO either mixing or reacting flows. Because of how this study's parameters were determined, the compressible flow module is more appropriate for our purposes here. 
The parameters determined for the compressible flow module take into account the chemistry of the reacting flow by solving for the individual specific heat ratio values. The compressible flow module allows the user to select different parameters, such as flow temperature, fluid velocity, and mass flow to name just a few. Some of these values can be found by using the NASA Combustion Equilibrium with Applications program, available online. This program allows users to calculate the chemical equilibrium production concentrations from any set of reactants (Zehe, 2010). It also helps determine the thermodynamic and transport properties for the product mixture, which, in this case, is the fuel and oxidizer combination used in the flow. The results from the use of the NASA CEA program online can be found in the appendix of this document.

The NASA CEA program provides a very important parameter: combined specific heat ratio per reactant. This value considers the chemical reaction and allows for quick changes across the different combinations in order to simplify the problem. Since the specific heat ratio term is fixed, compressible flow option is justifiable; it is assumed that the combustion in the chamber is in equilibrium, therefore the flow in the nozzle can be considered as a "frozen flow."

Once the flow has been selected, the following tab the Modeling Specifications (MO) can be selected and its specifications filled in. Figure 26 below shows the first vertical tab on the left hand side of the figure which is called the "Global" option followed by the flow option. The global tab allows for name change, and below it, the polar option allows the user to make the geometry either axisymmetric or nonaxisymmetric. In this case, the geometries have all been designed to be axisymmetric and 
the name has been changed to Stage1_LOX_RP1. The axisymmetric option makes use of the simplified contour and has the added benefit of reducing the complexity of the computation that was done for the particular grid. This is especially beneficial for machines that are not clustered, which is the case in this study. The next tab, called "Flow," allows the user to select the gas and viscous models to be used in the calculations. For these cases, an ideal gas model was chosen due to the fact that the flow being modeled is assumed to be in equilibrium and frozen. Within the architecture of CFD-FASTRAN-SOLVER, the selection of ideal gas implies that gas was of a single species. Thus, the assumption that the flow is in equilibrium must be enabled. The cases are therefore run with the mixing/reacting combustion flow as a single species gas. 


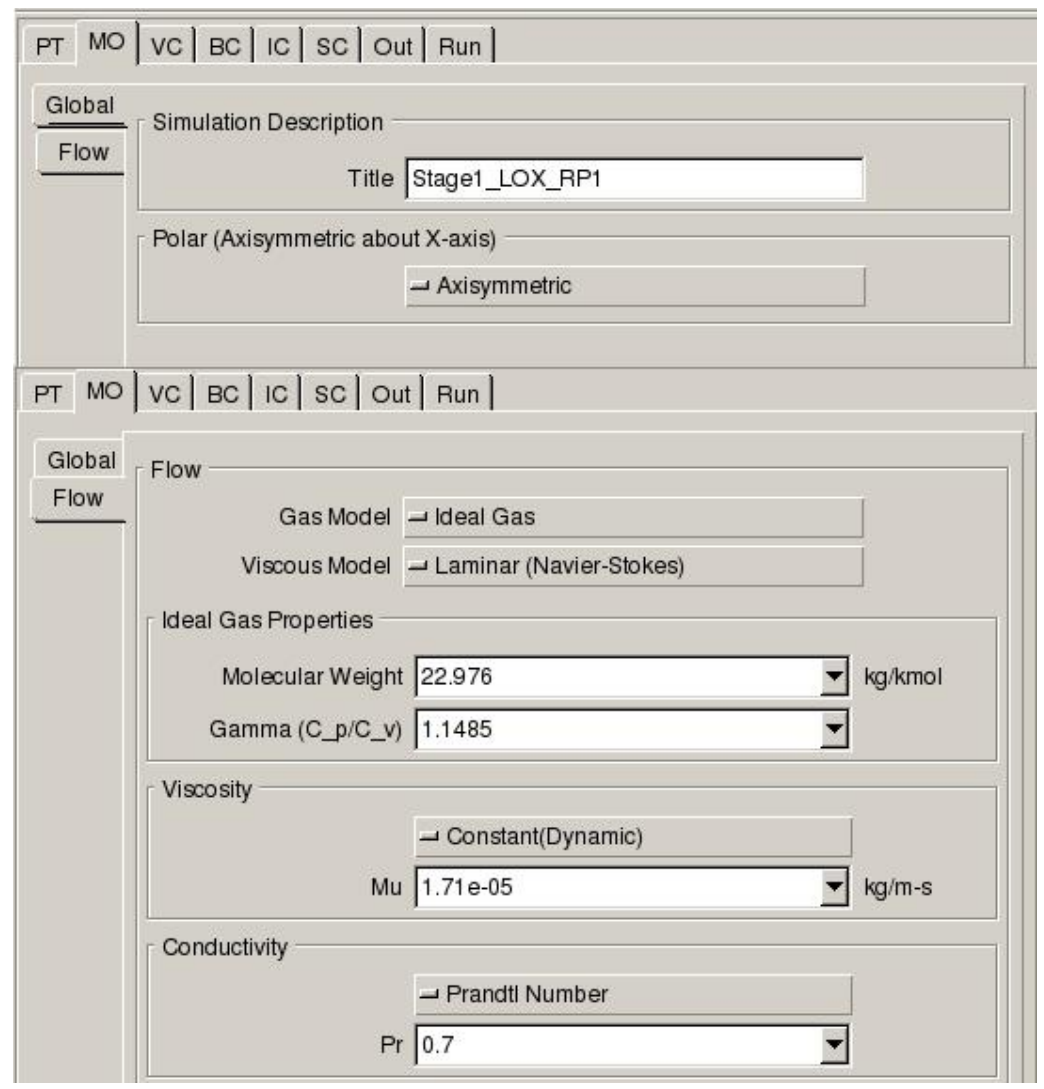

\section{Figure 26 MO tab display both the Global Options and Flow Selections}

Figure 26 above displays the values inserted for one of the cases and the naming of that case. The ideal gas properties of the molecular weight and specific heat ratio term were calculated in the NASA CEA program found online. This is how the assumption of a one species gas can be used with the ideal gas option enabled. The viscous model was chosen to be laminar Navier-Stokes because of the previously explained equilibrium flow assumption. The selection of the viscous model opens a new set of options for the ideal gas properties, viscosity, and flow conductivity settings. By selecting the laminar flow option, the solver is able to model the momentum and heat transport of the flow. The 
laminar flow option allows the solver to output the u direction velocity, $\mathrm{v}$ direction velocity, w direction velocity, pressure, and temperature. These parameters are necessary for comparing different cases. Furthermore, the solver will also output the calculated laminar viscosity and thermal conductivity. The viscosity value is left as default because of the previously stated assumptions regarding flow. The conductivity value is left at default because the Prandtl Number of 0.7 fits for most gases and is a good because the reaction in question is taking place in the atmosphere. The Prandtl number reflects the ability of a fluid to conduct heat in the thermal boundary layer versus its ability to transport momentum in the velocity boundary layer. This assumption implies that the gas is considered to be calorically perfect, or ideal, and that the gas is in thermal equilibrium. Figure 27 below features the Volume Conditions (VC) tab selection. The VC tab in this case is left as default because the properties being analyzed for all of the geometry zones are fluids. Selecting the fluid option allows the solver to apply flow equations to the selected volume, and in this case, all three separate zones. 


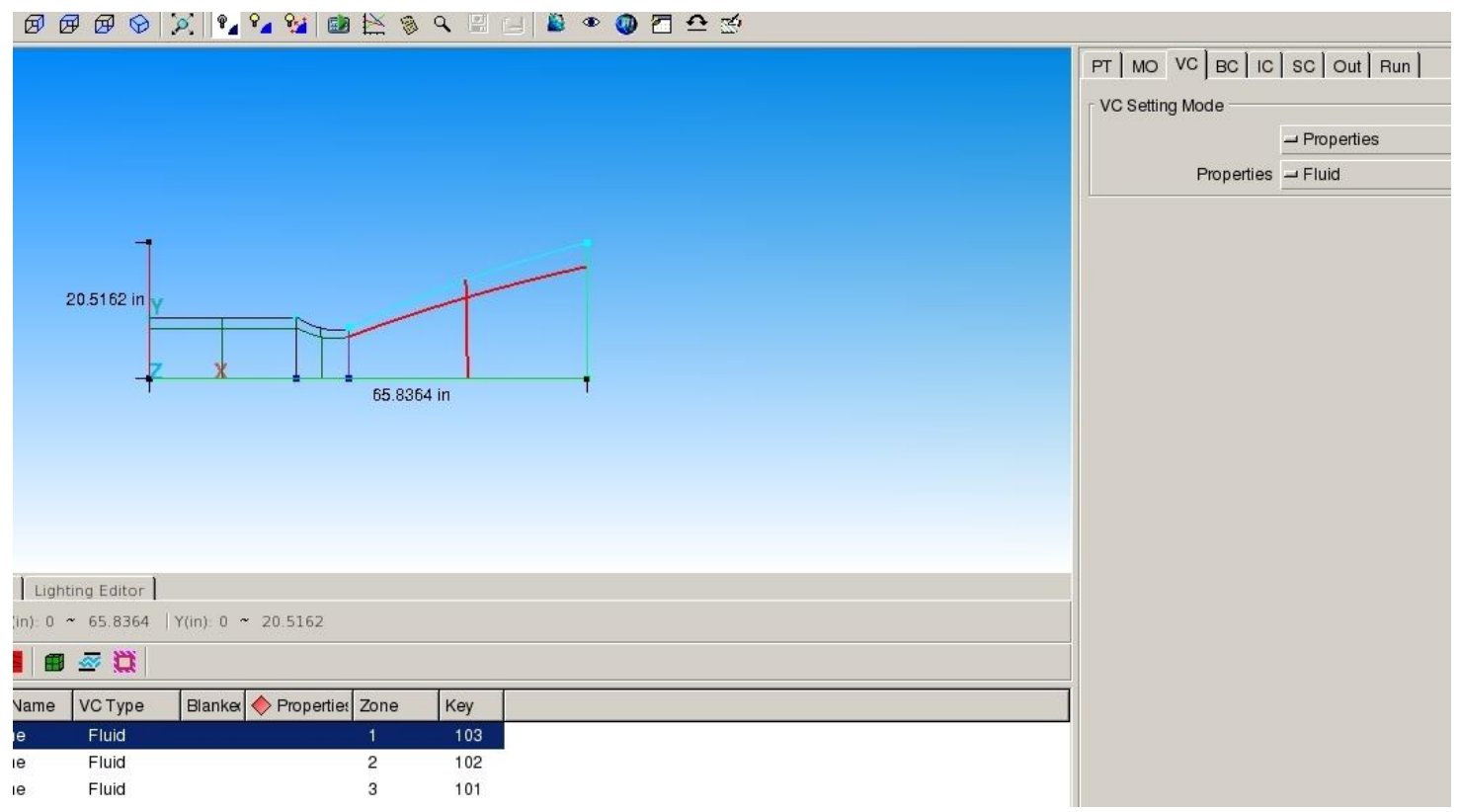

Figure 27 VC Module with Fluid Properties Selection

Next, the Boundary Conditions (BC) tab, which, in combination with the initial conditions, specifies the problem that needs to be solved, is discussed. The boundary conditions (part of the problem) help specify partial specifications for the model and the simulated environment. These conditions prescribe the fluid and the flow state at the boundaries of the imported model during the entire simulation time period. Figure 28 displays the $\mathrm{BC}$ tab and how the different geometry sections allow for different parameters of the boundary conditions. 


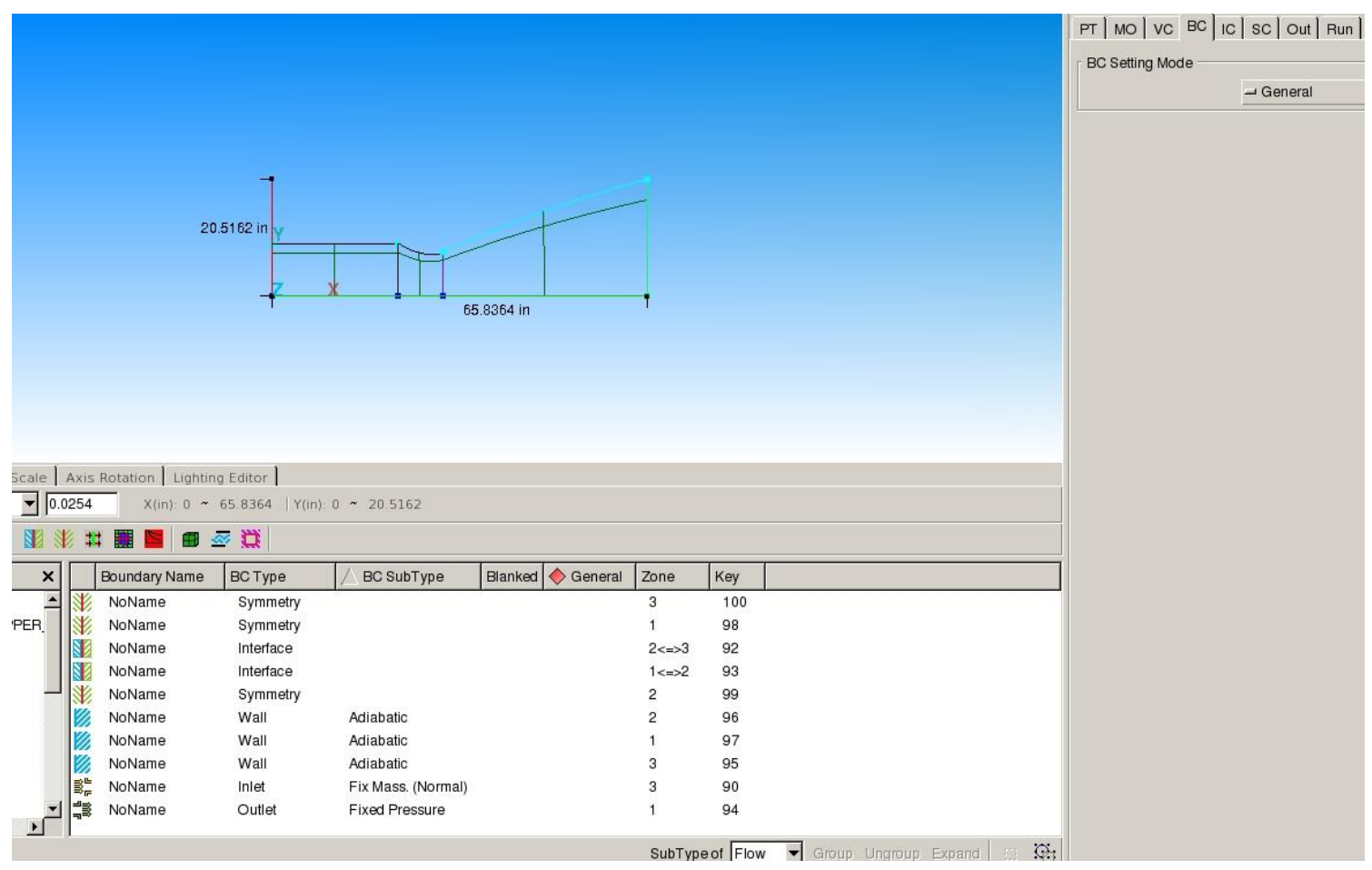

Figure 28 Boundary Conditions of the model

The lower center window displays a variety of geometry elements. Each part that comprises the geometry is listed here and allows for the selection of various boundary condition types and subtypes. The components of the model are generally determined in the CFD-GEOM stage of model development where all of the walls, lines of symmetry, interfaces, inlet, and boundary are specified. However, the components of the model can be changed within the solver GUI. The boundary condition subtypes help define what type of boundary condition is occurring at that particular location. For example, at the top of the chamber, inlet conditions that have a fixed mass flow are normal to the plane.

The boundary condition subtype selected for the inlet was a fixed mass flow rate, due to the previously stated assumptions. In addition, the boundary condition at the inlet 
is assumed to be a subsonic boundary due to the fact that the flow coming from the injectors was in the subsonic range. Furthermore, all of the information for the fixed mass option were all calculated in NASA CEA, or were known from literature research Figure 29 below displays the setting of the values that are known in the inlet subtype boundary condition window.

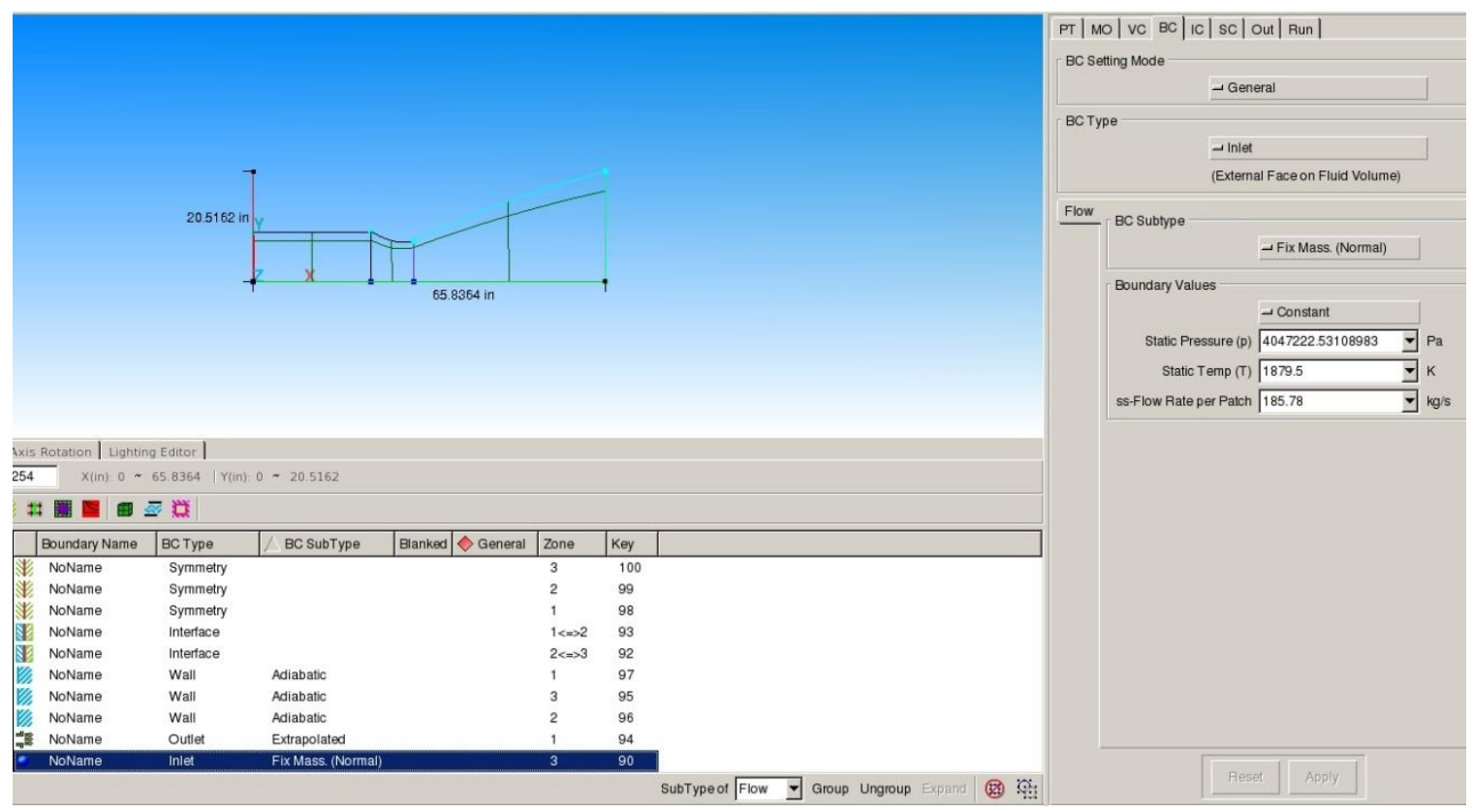

Figure 29 Inputs for the Inlet Boundary Condition Subtype

The outlet condition has two options: fixed pressure and the extrapolated condition. The extrapolated condition was chosen for this study because of the supersonic nature of the flow. Selecting this option results in variables that are extrapolated from the interior of the domain to the exit of the boundary. The wall boundary conditions are set at the default adiabatic setting because this option sets the surface heat flux to zero. This also assumes that the structural and flow temperatures 
eventually equilibrate, which means that that there is no flow in either direction of the boundary. For these cases, this is an adequate assumption because the cases are compared to each other when the flows are in steady engine operation.

The next tab to set up for the solver is the Initial Conditions (IC) tab. It is especially important that the user input physically possible and accurate values so that the time-accurate simulation can properly initialize. There are two main options for setting this section up: "Volume-by-Volume" or "For All Volumes." These two options refer to the setting of the initial conditions for either all of the zones or each of them individually. In these cases, the setting was selected to be "For All Volumes" and in the subsequent tab below the option the "Initial Condition From" was selected to be "Constant." Figure 30 displays the initial condition drop down selections for one of the cases in this study. The figure also shows the subsequent information that was added the empty fields that appeared.
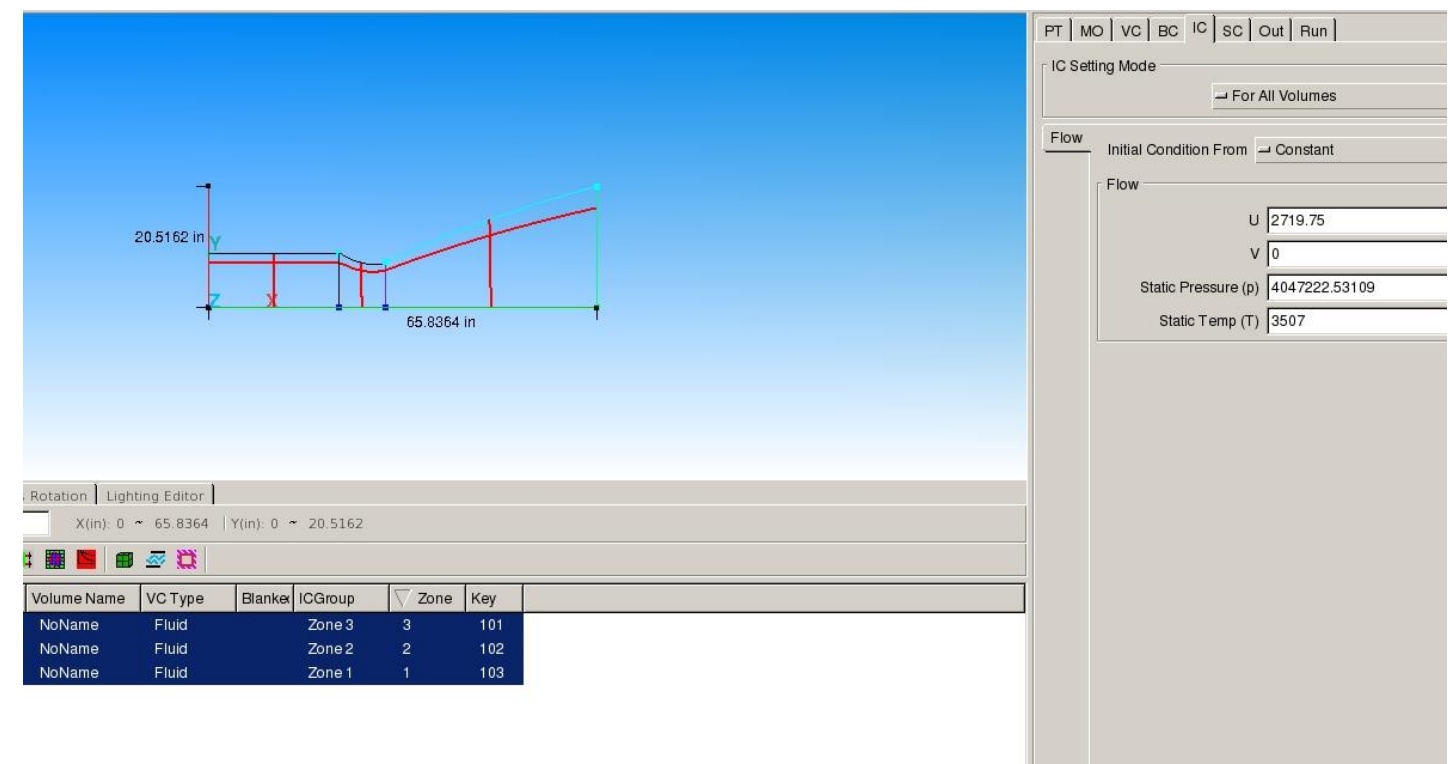

Figure 30 Example for setting of the Initial Boundary Conditions 
The next tab to set up is the Simulation Controls (SC) tab. This tab allows the user to modify settings that are related to the numerical time-integration and spatial discretization. It also allows the user to change other settings, such as how many cycles to run and what outputs to print. Figure 31 below displays the inputs that were modified in the sub-tabs in the main SC tab. The maximum number of cycles was set to 75000, as it was found through earlier setup tests on general cases for this study. The chosen number signifies the point at which the problem reaches steady state. Steady state in this reference is defined as the point at which solutions become steady in the residual results window of the solver. Essentially, reaching the steady state ensures that the flow is fully developed; therefore, the calculated result is as complete as possible for the inputs used. The other options that are shown for the later sub-tabs are left unchanged. 


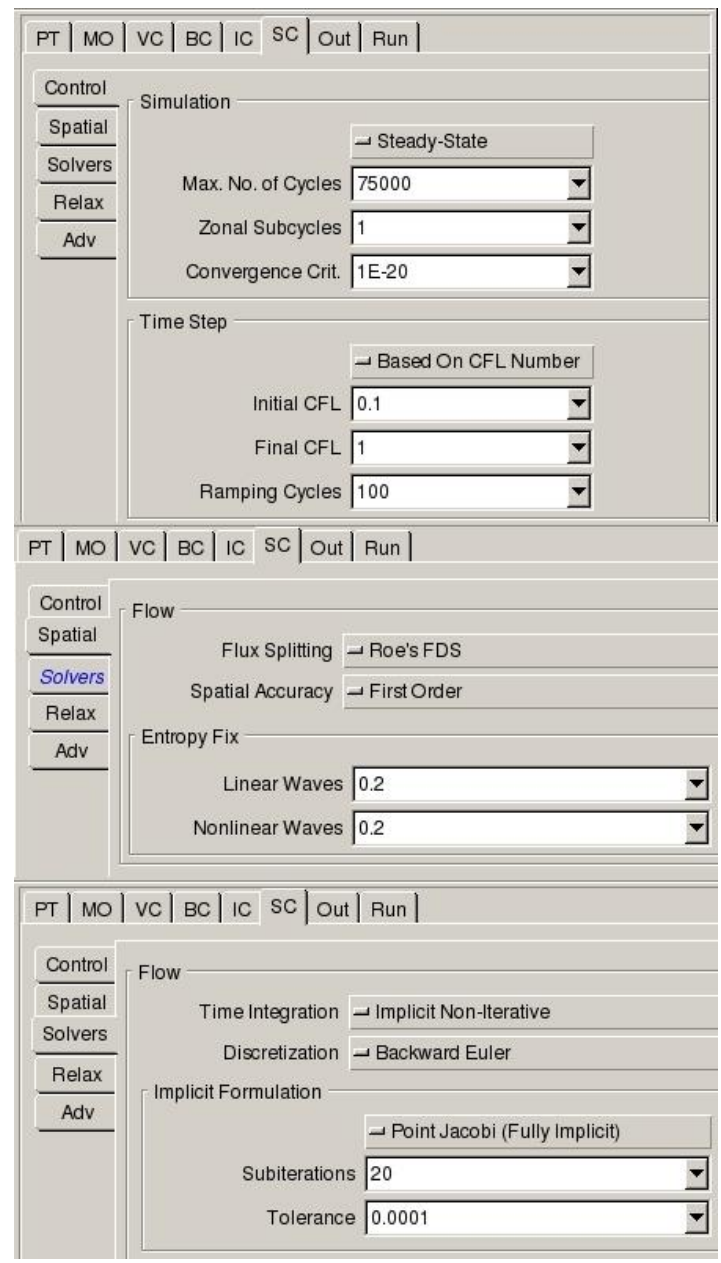

Figure 31 The sub-tabs under the SC tab that were modified

Figure 31 above displays the spatial sub-tab options near the top of the figure.

The options for flux splitting and spatial accuracy are, respectively, Roe's Flux Difference Scheme (FDS) and "First Order." Roe's FDS was left as the default option for problem solving flexibility by allowing some of the erroneous extrema into the flow solution. Selecting this option enables the "Entropy Fix" fields to be modified for the scheme. These values permit the increase in numerical dissipation of the scheme, thereby allowing the solution to converge more easily for difficult problems (Cfd-fastran 
overview). The values typically range from .1 to .3 , however, .2 is deemed as sufficient for most cases (Cfd-fastran overview). The "First Order" option was chosen when the flow conditions and behavior were unknown. The "First Order" option allows the user to run a particular geometry and still arrive at a solution. The other options in Figure 31 were left as default because the flow was able to reach convergence more easily. The main differences between the implicit and explicit options are related to the hardware limitations--specifically, memory limits--of the computer. Therefore, because this study utilized a single computer with limited memory, the default implicit non-iterative choice is attractive for generating solutions. This is the driving factor for the following selected options: Backward Euler method, Point Jacobi, and such following sub-tabs as "Relax."

The second to last tab that needs to be modified is the Output Panel (Out) tab. This tab allows the user to modify the output data from selecting the number of cycles to be printed in the output files. Among the sub tabs, the most important is the Print subtab. This tab allows the user to set up the "Aero Force Summary," which generates the forces that are calculated by the solver in a separate file. Then, in this area, the user can select the zones that were recorded. In these cases, all of the zones were recorded for completeness. This is important because the user will pull the data for various parameters from these generated files.

Finally, the last tab to be modified is the Run Panel (Run) tab. This tab grants the user control over the progress of both, the solution and the solver. Furthermore, here a user can start and/or stop a solution and save it. This tab allows a user to view the output file to help troubleshoot any errors that may occur during initial setups. Finally, the 
"View Residual" option in the Monitor section of the tab is important because it allows the user to view the progress of the cycles that are being conducted. Figure 32 below displays a typical sample residual output.

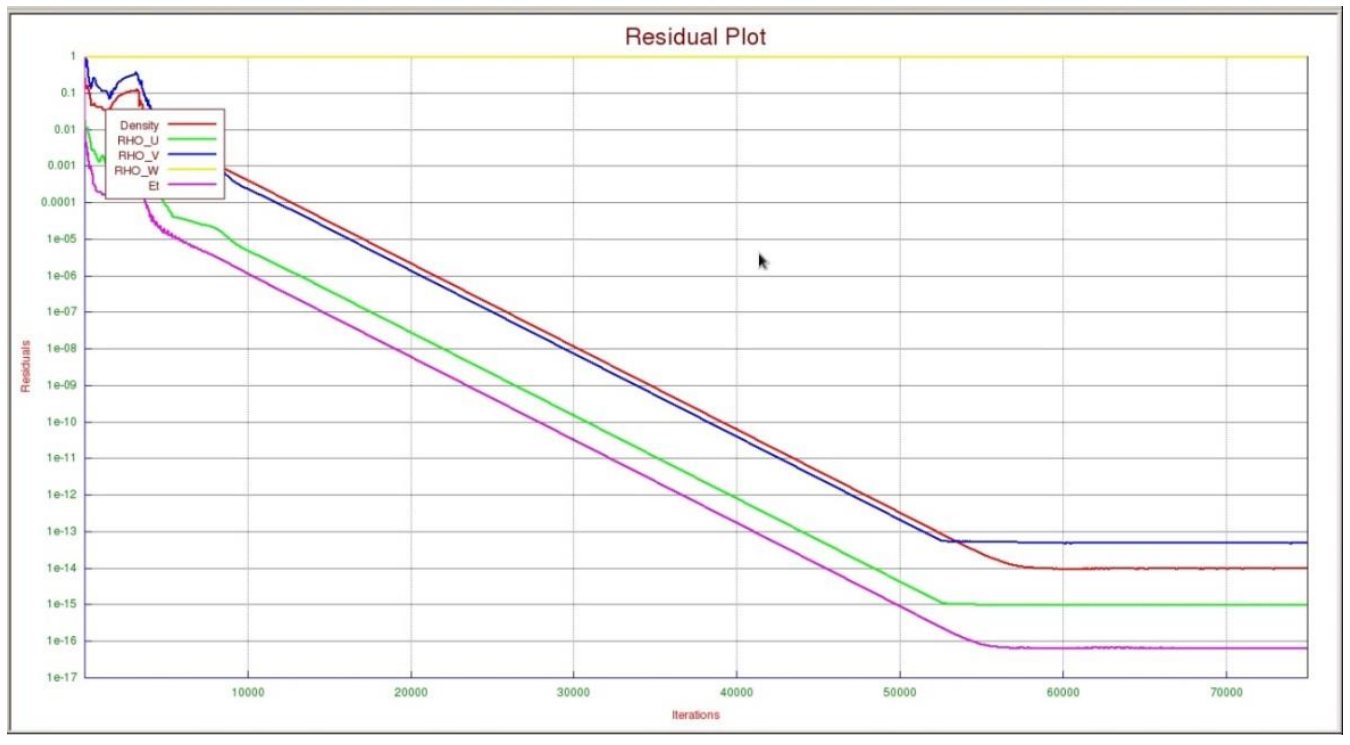

Figure 32 Sample Residual Output

\section{Experimental Procedure}

The experimental runs for this study were split into two main iterations. The first iteration focused on changing the reactant combinations, whereas the second iteration focused on the same parameters as the first, but increased the pressure for all of the engines. The intent was to determine which changes created the most desirable results in terms of thrust and ISP. Essentially, this study developed a clear line of analysis to demonstrate from where conclusions may be derived. The sets of iterations were split up according to Table 3 and Table 4 displayed below. This table is a roadmap for the analysis in this study. 
Table 3 Table of Inputs and Parameters for Iteration One

\begin{tabular}{|c|c|c|c|c|c|c|c|c|}
\hline \multirow{2}{*}{\multicolumn{3}{|c|}{$\frac{\text { Stage }}{\text { Reactants }}$}} & \multicolumn{3}{|c|}{ I } & \multicolumn{3}{|c|}{ II } \\
\hline & & & LOX/RP1 & LOX/LH2 & LF2/LH2 & LOX/RP1 & LOX/LH2 & LF2/LH2 \\
\hline \multicolumn{3}{|c|}{ Flow Option } & \multicolumn{6}{|c|}{ Compressible Flow } \\
\hline \multicolumn{3}{|c|}{ Molecular Weight } & 23 & 6.55 & 5.86 & 23 & 6.55 & 5.86 \\
\hline \multicolumn{3}{|c|}{ Gamma } & 1.15 & 1.27 & 1.32 & 1.15 & 1.27 & 1.32 \\
\hline \multicolumn{3}{|c|}{ Gas Model } & \multicolumn{6}{|c|}{ Ideal Gas } \\
\hline \multicolumn{3}{|c|}{ Viscous Model } & \multicolumn{6}{|c|}{ Laminar (Navier-Stokes) } \\
\hline \multicolumn{3}{|c|}{ Polar Option } & \multicolumn{6}{|c|}{ Axisymmetric } \\
\hline \multicolumn{3}{|c|}{ Volume Conditions } & \multicolumn{6}{|c|}{ Fluid } \\
\hline \multirow{7}{*}{ 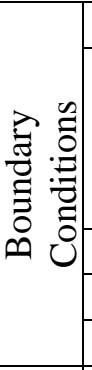 } & \multicolumn{2}{|c|}{ Wall } & \multicolumn{6}{|c|}{ Adiabatic } \\
\hline & \multirow{3}{*}{$\frac{\vec{\omega}}{\Xi}$} & Pressure & \multicolumn{3}{|c|}{$587 \mathrm{psi}$} & \multicolumn{3}{|c|}{$682 \mathrm{psi}$} \\
\hline & & Temp. & $3507 \mathrm{~K}$ & $1974.8 \mathrm{~K}$ & $1879.5 \mathrm{~K}$ & $3502.3 \mathrm{~K}$ & $1974.5 \mathrm{~K}$ & $1879 \mathrm{~K}$ \\
\hline & & $\begin{array}{l}\text { Mass } \\
\text { Flow }\end{array}$ & $\begin{array}{c}244.6 \\
\mathrm{~kg} / \mathrm{m} 3\end{array}$ & $\begin{array}{r}186.3 \\
\mathrm{~kg} / \mathrm{m} 3\end{array}$ & $\begin{array}{r}185.3 \\
\mathrm{~kg} / \mathrm{m} 3\end{array}$ & $\begin{array}{r}116.9 \\
\mathrm{~kg} / \mathrm{m} 3\end{array}$ & $\begin{array}{c}90.5 \\
\mathrm{~kg} / \mathrm{m} 3\end{array}$ & $\begin{array}{c}90.6 \\
\mathrm{~kg} / \mathrm{m} 3\end{array}$ \\
\hline & \multicolumn{2}{|c|}{ Outlet } & \multicolumn{6}{|c|}{ Extrapolated } \\
\hline & \multicolumn{2}{|c|}{ Symmetry } & \multicolumn{6}{|c|}{ Default } \\
\hline & \multicolumn{2}{|c|}{ Interface } & \multicolumn{6}{|c|}{ Default } \\
\hline \multirow{3}{*}{ 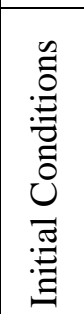 } & \multicolumn{2}{|c|}{ U Velocity } & $\begin{array}{c}2728.4 \\
\mathrm{~m} / \mathrm{s}\end{array}$ & $\begin{array}{c}3582.4 \\
\mathrm{~m} / \mathrm{s}\end{array}$ & $\begin{array}{c}3600.7 \\
\mathrm{~m} / \mathrm{s}\end{array}$ & $\begin{array}{c}2765.9 \\
\mathrm{~m} / \mathrm{s}\end{array}$ & $\begin{array}{c}3625.7 \\
\mathrm{~m} / \mathrm{s}\end{array}$ & $\begin{array}{c}3642.6 \\
\mathrm{~m} / \mathrm{s}\end{array}$ \\
\hline & \multicolumn{2}{|c|}{$\begin{array}{c}\text { Static } \\
\text { Pressure }\end{array}$} & \multicolumn{3}{|c|}{$587 \mathrm{psi}$} & \multicolumn{3}{|c|}{$682 \mathrm{psi}$} \\
\hline & \multicolumn{2}{|c|}{ Static Temp. } & $3507 \mathrm{~K}$ & $1974.8 \mathrm{~K}$ & $1879.5 \mathrm{~K}$ & $3502.3 \mathrm{~K}$ & $1974.5 \mathrm{~K}$ & $1879 \mathrm{~K}$ \\
\hline \multicolumn{3}{|c|}{$\begin{array}{l}\text { Solver } \\
\text { Control }\end{array}$} & \multicolumn{6}{|c|}{75000} \\
\hline
\end{tabular}


Table 4 Table of Inputs and Parameters for Iteration Two

\begin{tabular}{|c|c|c|c|c|c|c|c|c|}
\hline \multicolumn{3}{|c|}{ Stage } & \multicolumn{3}{|c|}{$\mathrm{I}$} & \multicolumn{3}{|c|}{ II } \\
\hline \multicolumn{3}{|c|}{ Reactants } & LOX/RP1 & LOX/LH2 & LF2/LH2 & LOX/RP1 & LOX/LH2 & LF2/LH2 \\
\hline \multicolumn{3}{|c|}{ Flow Option } & \multicolumn{6}{|c|}{ Compressible Flow } \\
\hline \multicolumn{3}{|c|}{ Molecular Weight } & 23 & 6.55 & 5.86 & 23 & 6.55 & 5.86 \\
\hline \multicolumn{3}{|c|}{ Gamma } & 1.15 & 1.27 & 1.32 & 1.15 & 1.27 & 1.32 \\
\hline \multicolumn{3}{|c|}{ Gas Model } & \multicolumn{6}{|c|}{ Ideal Gas } \\
\hline \multicolumn{3}{|c|}{ Viscous Model } & \multicolumn{6}{|c|}{ Laminar (Navier-Stokes) } \\
\hline \multicolumn{3}{|c|}{ Polar Option } & \multicolumn{6}{|c|}{ Axisymmetric } \\
\hline \multicolumn{3}{|c|}{ Volume Conditions } & \multicolumn{6}{|c|}{ Fluid } \\
\hline \multirow{7}{*}{ 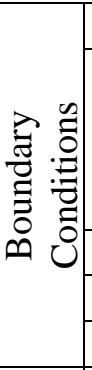 } & \multicolumn{2}{|c|}{ Wall } & \multicolumn{6}{|c|}{ Adiabatic } \\
\hline & \multirow{3}{*}{$\stackrel{\vec{\omega}}{\Xi}$} & Pressure & \multicolumn{6}{|c|}{$1000 \mathrm{psi}$} \\
\hline & & Temp. & $3545 \mathrm{~K}$ & $1975 \mathrm{~K}$ & $1879.5 \mathrm{~K}$ & $3545 \mathrm{~K}$ & $1975 \mathrm{~K}$ & $1879 \mathrm{~K}$ \\
\hline & & Mass & $\begin{array}{c}231.4 \\
\mathrm{~kg} / \mathrm{m} 3\end{array}$ & $\begin{array}{c}178.4 \\
\mathrm{~kg} / \mathrm{m} 3\end{array}$ & $\begin{array}{c}185.3 \\
\mathrm{~kg} / \mathrm{m} 3\end{array}$ & $\begin{array}{c}113.3 \\
\mathrm{~kg} / \mathrm{m} 3\end{array}$ & $\begin{array}{c}88.6 \\
\mathrm{~kg} / \mathrm{m} 3\end{array}$ & $\begin{array}{c}88.98 \\
\mathrm{~kg} / \mathrm{m} 3\end{array}$ \\
\hline & \multicolumn{2}{|c|}{ Outlet } & \multicolumn{6}{|c|}{ Extrapolated } \\
\hline & \multicolumn{2}{|c|}{ Symmetry } & \multicolumn{6}{|c|}{ Default } \\
\hline & \multicolumn{2}{|c|}{ Interface } & \multicolumn{6}{|c|}{ Default } \\
\hline \multirow{3}{*}{ 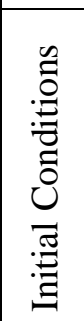 } & \multicolumn{2}{|c|}{ U Velocity } & $\begin{array}{c}2883.9 \\
\mathrm{~m} / \mathrm{s}\end{array}$ & $\begin{array}{c}3737.3 \\
\mathrm{~m} / \mathrm{s}\end{array}$ & $\begin{array}{c}3600.7 \\
\mathrm{~m} / \mathrm{s}\end{array}$ & $\begin{array}{c}2884.9 \\
\mathrm{~m} / \mathrm{s}\end{array}$ & $\begin{array}{c}3737.3 \\
\mathrm{~m} / \mathrm{s}\end{array}$ & $\begin{array}{c}3745.7 \\
\mathrm{~m} / \mathrm{s}\end{array}$ \\
\hline & \multicolumn{2}{|c|}{$\begin{array}{c}\text { Static } \\
\text { Pressure }\end{array}$} & \multicolumn{6}{|c|}{$1000 \mathrm{psi}$} \\
\hline & \multicolumn{2}{|c|}{ Static Temp. } & $3545 \mathrm{~K}$ & $1975 \mathrm{~K}$ & $1879.5 \mathrm{~K}$ & $3545 \mathrm{~K}$ & $1975 \mathrm{~K}$ & $1879.5 \mathrm{~K}$ \\
\hline \multicolumn{3}{|c|}{$\begin{array}{c}\text { Solver } \\
\text { Control }\end{array}$} & \multicolumn{6}{|c|}{75000} \\
\hline
\end{tabular}


Table 3 and Table 4 display all of the inputs and parameters that will need to be altered for each of the stages, as well as the individual reactant mixtures for the study. This comprehensive table helps to keep track of which parameters affect the performance parameters of the engines. Most of these values have been determined beforehand through side calculations completed in either the EXCEL calculator (mentioned in previous sections) or the NASA CEA online program. The NASA CEA outputs for the temperature, molecular weight, and gamma terms can be found in the appendix.

All of these runs were completed in the Aerospace Engineering CFD Lab provided by San José State University. There are a total of six ESI CFD Suite configured computers that are available for use between regularly scheduled courses that occupy the classroom. Each of these computers was set up accordingly to run one case in one of the iterations listed above. Then the computer will run a second case. Previous experience using the computer systems has revealed that runs of this complexity take approximately 18 to 20 hours each. The advantage of using multiple computers is that it greatly reduces the time spent waiting for runs to be finished; thus, more data are generated in a shorter amount of time. The spreading of the computations is also advantageous in that it allows for a quick turnaround in correcting any mishaps that may arise. Therefore, this plan is ideal for the time constraints per case.

Table 5 displays data at normal conditions for the different propellant choices found in the reference by Huzel and Huang (1992). This table serves to display the general liquid characteristics of the propellants at normal conditions. These normal conditions are defined as the standard handling conditions of the propellants in regards to 
propulsion system usage. These data are particularly useful in understanding how the system is affected upstream of the feed system. This shows the relationship between the system and how the turbo pump would need to be redesigned or modified in order to operate with these propellants.

Table 5 Propellant Data at Normal Conditions (Huzel \& Huang, 1992)

\begin{tabular}{|c|c|c|c|c|}
\hline Liquid & Temperature, $\mathrm{F}$ & Vapor Pressure, $\mathrm{psia}$ & Density, $\mathrm{lb} / \mathrm{ft}^{3}$ & Viscosity, $\mathrm{lb}^{-} / \mathrm{in}^{2}$ \\
\hline $\mathrm{LO}_{2}$ & -297.6 & 14.7 & 71.17 & $0.28 \times 10^{-7}$ \\
\hline $\mathrm{LF}_{2}$ & -307 & 14.7 & 94.21 & $0.35 \times 10^{-7}$ \\
\hline $\begin{array}{c}\mathrm{RP}-1 \\
\text { Kerosene })\end{array}$ & 60 & 14.7 & $49.8-50.8$ & $3.22 \times 10^{-7}$ \\
\hline $\mathrm{LH}_{2}$ & -422.9 & .031 & 4.43 & $0.02 \times 10^{-7}$ \\
\hline
\end{tabular}

Finally, determining which is the "best" option or alteration to the rocket engines was conducted through a combination of maximizing the ISP and thrust. Furthermore, analyzing the flow was important as it will give insight to any possible instabilities such as non-choked flow, possibilities for vibration, and so on. These are all factors that are not necessarily taken into account by the CFD solver, but must be conceptualized and noted. 
Oxidizer Options. The main two options being considered in this study for an oxidizer in the Titan I propulsion system include liquid oxygen and fluorine. These two options are considered because of their ability to generate high ISP in a rocket combustion system, as well as their history in other high thrust systems. Furthermore, because of the nature of this study, improvements to the system are driven by ISP performance and overall possible increases in either range or mass-to-orbit results through higher thrust.

Liquid Oxygen. Liquid oxygen is used commonly in rocket systems as an oxidizer because of its ability to create a high specific impulse when reacted with other fuels (Huzel \& Huang, pp. 20-22). Its legacy with high thrust systems, such as the SSME, is a prominent upside. The main downside of this oxidizer is the fact that it requires a rather complex infrastructure for storage, transference, and use due to its very low cryogenic temperatures. It is for this main reason that the Titan II iteration of the Titan family moved away from the use of liquid oxygen. Furthermore, when implemented in a rocket propulsion system, extra mass is usually required to be able to properly utilize this oxidizer. In the long run, if a system is not properly designed, the mass-to-weight ratio can be affected adversely. 
Liquid Fluorine. Liquid fluorine is infrequently used in rocket systems as an oxidizer because of the difficulty of handling this chemical, its toxicity, and its volatile reactions with almost anything except lighter noble gases (Huzel \& Huang, pp. 18-22). However, its performance when used in combination with other fuel options in this study is impressive. Liquid fluorine's low molecular weight allows it to generate impressively high potential exit velocities. This oxidizer can also be used in a cryogenic system. Essentially, if its use is determined to positively outweigh the negatives, only small alterations would need to be made to the propulsion system in order for this oxidizer to be implemented. Compared to liquid oxygen, this oxidizer type will very likely increase the overall efficiency of the propulsion system.

Fuel Options. The two main fuel options in this study to be used in the Titan I propulsion system include kerosene and hydrogen. These options are widely available and have been used in such regeneratively-cooled systems as the Titan I. This cooling system uses these particular fuel types to transfer heat from the chamber to the coolant because of their differences in heat transfer coefficients and thermal conductivity. Contemporarily, kerosene, which was used originally in the Titan I, and hydrogen are resultantly more widely used. 
Kerosene. Kerosene is a chemical mixture of different hydrocarbons; its chemical makeup depends on its source, which is most similar to jet fuel (Hill \& Peterson, 1992). The military uses a special type of kerosene known as Refined Petroleum 1, or more commonly RP-1, which differs in molecular weight values. For this study, the standard set molecular weight value used in NASA CEA was 23. When mixed with liquid oxygen, reasonable ISP values are generated, which can be seen in the published data of the Titan I. An advantage of using kerosene is that it does not require significant equipment for handling and use in a propulsion system. It can also be used with varying effect in regeneratively cooled engines such as the Titan I.

Liquid Hydrogen. Liquid Hydrogen is commonly used in such high performance engines such as the Space Shuttle Main Engine (SSME), or the J-2X, due to its ability to deliver significantly higher specific impulse than other rocket fuels. Although liquid hydrogen is strongly desired as a fuel, it does have a variety of drawbacks. The drawbacks mainly stem from the fact that it does not store well over long periods of time; hence, it is not used in the military for ballistic systems. Furthermore, this fuel requires an extensive infrastructure to store, transfer, and use in terms of plumbing because of its chemical nature, such a small molecule to contain as it is. However, this study is analyzing performance enhancements of the Titan I with applications, and thus, it is still a strong contender as a replacement for kerosene. 


\section{Boundary Conditions}

The number of zones, size of grid, and complexity are all determined prior to setting the boundary conditions, which, for this study, have been centered on the required inputs of the ESI FASTRAN solver. These boundary conditions stem from the initial geometry and grid model setups. This is evident in the creation of three zones, one for each of the major components of the rocket engine. These main components are: the chamber, throat, and nozzle regions. The boundary conditions will each be applied to these separate zones.

Initially, the study considers no-slip conditions within a compressible laminar flow. The no-slip condition applies to viscous fluids and interactions with a solid boundary (Cfd-fastran overview, 2011). Essentially, when a viscous fluid interacts with a surface, the fluid will have zero velocity relative to the boundary. Laminar flow can be described as "parallel flow," meaning that the flow behaves like orderly streamlines, without perpendicular cross currents to the flow, or any other type of disruption (Bird, 1994). Although this condition drastically reduces solution time, it is an inaccurate representation of realistic flow within the systems being analyzed. It does, however, help provide a good academic starting point for understanding the processes within the studied rocket engines.

The main boundary conditions modified in each study are found at the inlet, outlet, and walls of the geometry. The regions within each of the dedicated rocket engine zones are modified with all of the available information on hand from the literature 
review as well as from side calculations conducted in the EXCEL calculator, a sample of which can be found in the appendix. The following two tables display each run's different inlet, outlet, and wall conditions that were applied throughout the study. 
Table 6 Iteration 1 Boundary Conditions

\begin{tabular}{|c|c|c|c|c|c|c|c|c|c|c|}
\hline \multirow{2}{*}{\multicolumn{3}{|c|}{$\frac{\text { Stage }}{\text { Reactants }}$}} & \multicolumn{4}{|c|}{ I } & \multicolumn{4}{|c|}{ II } \\
\hline & & & LOX/RP1 & LOX & & LF2/LH2 & LOX/RP1 & LOX & $\mathrm{H} 2$ & LF2/LH2 \\
\hline \multirow{7}{*}{ 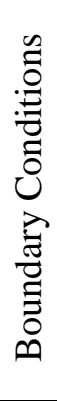 } & \multicolumn{2}{|c|}{ Wall } & \multicolumn{8}{|c|}{ Adiabatic } \\
\hline & \multirow{3}{*}{$\frac{\overrightarrow{0}}{\Xi}$} & Pressure & \multicolumn{4}{|c|}{$587 \mathrm{psi}$} & \multicolumn{4}{|c|}{$682 \mathrm{psi}$} \\
\hline & & Temp. & $3507 \mathrm{~K}$ & $\begin{array}{c}1974.8 \\
\mathrm{~K} \\
\end{array}$ & \multicolumn{2}{|c|}{$1879.5 \mathrm{~K}$} & $3502.3 \mathrm{~K}$ & $\begin{array}{c}1974.5 \\
\mathrm{~K} \\
\end{array}$ & \multicolumn{2}{|c|}{$1879 \mathrm{~K}$} \\
\hline & & $\begin{array}{l}\text { Mass } \\
\text { Flow }\end{array}$ & $\begin{array}{r}244.6 \\
\mathrm{~kg} / \mathrm{m} 3\end{array}$ & $\begin{array}{c}186.3 \\
\mathrm{~kg} / \mathrm{m} 3\end{array}$ & \multicolumn{2}{|c|}{$185.3 \mathrm{~kg} / \mathrm{m} 3$} & $\begin{array}{r}116.9 \\
\mathrm{~kg} / \mathrm{m} 3\end{array}$ & $\begin{array}{c}90.5 \\
\mathrm{~kg} / \mathrm{m} 3\end{array}$ & \multicolumn{2}{|c|}{$90.6 \mathrm{~kg} / \mathrm{m} 3$} \\
\hline & \multicolumn{2}{|c|}{ Outlet } & \multicolumn{8}{|c|}{ Extrapolated } \\
\hline & \multicolumn{2}{|c|}{ Symmetry } & \multicolumn{8}{|c|}{ Default } \\
\hline & \multicolumn{2}{|c|}{ Interface } & \multicolumn{8}{|c|}{ Default } \\
\hline
\end{tabular}

Table 7 Iteration 2 Boundary Conditions

\begin{tabular}{|c|c|c|c|c|c|c|c|c|}
\hline \multirow{2}{*}{\multicolumn{3}{|c|}{$\begin{array}{c}\text { Stage } \\
\text { Reactants }\end{array}$}} & \multicolumn{3}{|c|}{ I } & \multicolumn{3}{|c|}{ II } \\
\hline & & & LOX/RP1 & LOX/LH2 & LF2/LH2 & LOX/RP1 & LOX/LH2 & LF2/LH2 \\
\hline \multirow{8}{*}{ 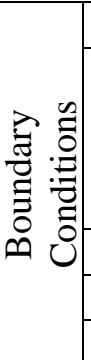 } & \multicolumn{2}{|c|}{ Wall } & \multicolumn{6}{|c|}{ Adiabatic } \\
\hline & \multirow{4}{*}{$\stackrel{\overrightarrow{0}}{\Xi}$} & Pressure & \multicolumn{6}{|c|}{$1000 \mathrm{psi}$} \\
\hline & & Temp. & $3545 \mathrm{~K}$ & $1975 \mathrm{~K}$ & $1879.5 \mathrm{~K}$ & $3545 \mathrm{~K}$ & $1975 \mathrm{~K}$ & $1879 \mathrm{~K}$ \\
\hline & & Mass & 231.4 & 178.4 & 185.3 & 113.3 & 88.6 & 88.98 \\
\hline & & Flow & $\mathrm{kg} / \mathrm{m} 3$ & $\mathrm{~kg} / \mathrm{m} 3$ & $\mathrm{~kg} / \mathrm{m} 3$ & $\mathrm{~kg} / \mathrm{m} 3$ & $\mathrm{~kg} / \mathrm{m} 3$ & $\mathrm{~kg} / \mathrm{m} 3$ \\
\hline & \multicolumn{2}{|c|}{ Outlet } & \multicolumn{6}{|c|}{ Extrapolated } \\
\hline & \multicolumn{2}{|c|}{ Symmetry } & \multirow{2}{*}{\multicolumn{6}{|c|}{ Default }} \\
\hline & \multicolumn{2}{|c|}{ Interface } & & & & \multicolumn{3}{|c|}{ Default } \\
\hline
\end{tabular}




\section{Chapter 3 Results}

The results presented in this section are all of the data generated by the ESI solver in truncated form. Samples of the output files for the forces present on the considered geometries are in the appendix. Other outputs, such as the flow field properties, are also displayed in graphical form for each of the individual runs in each iteration. The other data presented includes manipulated and transformed results from the analysis of the output data. These were presented in tables and graphs to demonstrate trends and display pertinent findings from the study.

Due to the importance of the geometry in this study, careful attention was given to the generation of the geometry. This, in turn, led to the generation of a calculated contour from known data and calculated data by using a variety of sources, such as NASA CEA, to find some of the required unknowns. Although two sets of geometries were created, only the one from calculated data was used. All efforts were made to make the calculated contours as close as possible to the originally published data contours that were traced from a blueprint found in the History of Rocketry and Astronautics by Murphy reference. This is relevant because the nearer to the original contours the higher the probability that the flows generated was correctly replicate those from the Titan I engines. Figure 33 and Figure 34 both show the superimposed 3D representation of the calculated geometry to the original published contours. The blue color represents the original contour from the published data that was traced. The red color represents the 
calculated nozzle from the published data. The values in the figures that correspond for each nozzle exit diameter are color coded and follow the convention mentioned earlier. Furthermore, these values are posted as a reference.

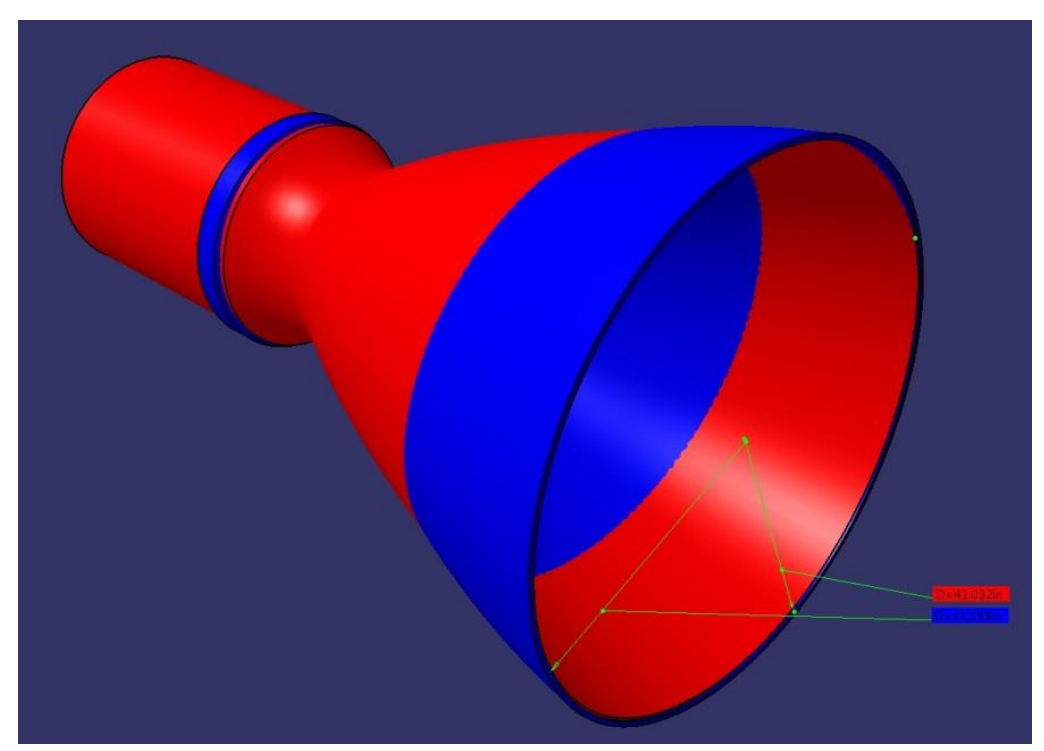

Figure 33 Stage One Differences in the Contours

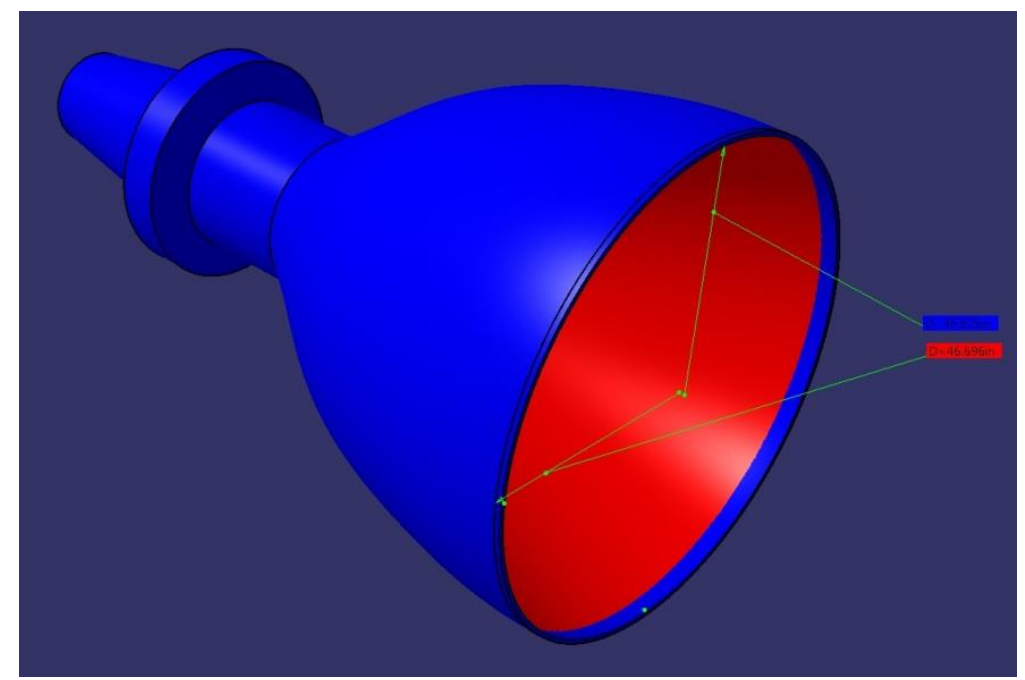

Figure 34 Stage Two Differences in the Contours 
From a visual inspection, it seems from Figure 33 that the contour of the nozzle portion is slightly off with very minimal differences in the curvature from the top to the bottom. Only the top part is larger while the bottom is smaller than the original. It is apparent that the chambers vary, as shown in Figure 34. This difference was caused by the fact that the contour provided represented the outside of the engine, and the contour that was calculated represents the interior. More critically, sections of the throat and nozzle vary somewhat, due to the similar reasons. Table 8 displays the parameters that were published alongside those that were calculated. It is important to note that the sea level thrust for the calculated portion of the table were found after analyzing the CFD results and were placed in the table for reference. A discussion of how these values were found will follow shortly. Table 9 displays the percent difference found for each of the parameters in the previous table; this is presented here in order to demonstrate the closeness of the contours.

\section{Table 8 Comparison Between Published and Calculated Data}

\begin{tabular}{|c|c|c|c|c|}
\hline Parameter & \multicolumn{2}{|c|}{ Published } & \multicolumn{2}{|c|}{ Calculated } \\
\hline Stage & I & II & I & II \\
\hline Area Throat, in ${ }^{2}$ & 181.9 & 66.73 & 165.24 & 67.93 \\
\hline Nozzle Diameter, in & 41.3 & 46.1 & 41.03 & 46.49 \\
\hline Nozzle Length, in & 36.5 & 46 & 37.3 & 46 \\
\hline Sea Level ISP, $\mathrm{s}$ & 251.9 & $283 *$ & 277 & 310.42 \\
\hline Sea Level Thrust, lbf & 300000 & $72896^{*}$ & 306328 & 67448 \\
\hline
\end{tabular}

*Note: Sea Level ISP and Thrust for Stage II were calculated from published data (Stumpf, 2000) and used only one calculated variable (Velocity at the Exit) to determine these values for completeness 
Table 9 Percent Difference Between Published and Calculated Data

\begin{tabular}{|c|c|c|}
\hline Parameter & Published & Calculated \\
\hline Stage & I & II \\
\hline Area Throat, in2 & $9.59 \%$ & $1.78 \%$ \\
\hline Nozzle Diameter, in & $0.66 \%$ & $1.06 \%$ \\
\hline Nozzle Length, in & $2.17 \%$ & $0.00 \%$ \\
\hline Sea Level ISP, $\mathrm{s}$ & $9.49 \%$ & $9.24 \%$ \\
\hline Sea Level Thrust, lbf & $2.09 \%$ & $7.76 \%$ \\
\hline
\end{tabular}

Table 9 above displays the percent difference for each of the parameters. As it can be seen, the differences are all below 10\%. The higher percentages can be attributed to the fact that the original contours were of the outside of the engines rather than the inside, and coupled with the varying wall thickness, this would generate large differences. The $0 \%$ difference in nozzle length for stage two for the calculated contour is considered an anomaly, and is attributed to calculator modifications during contour matching. The difference in sea level ISPs may be due to the fact that the calculated inputs may not have replicated the same atmospheric conditions as the original's during launch. Furthermore, the equations used to find the calculations are ideal in nature and have little grounding in reality. An engineering equation can only predict a real world result to a certain point. The sea level ISP and thrust for the first stage engine needed to be back-calculated for comparison, because these factors were not originally published. This was done in this study for the sake of completeness and comparison.

To find the sea level ISP for Stage II, the ISP seen in Equation 8 was used to plot points at different altitudes with diminishing gravity. After solving for the change in gravity, and researching the engine's mass flow rate of the as well as the trajectory peak found in the Stumpf reference, calculating the ISP at sea level became a simple problem. 
Figure 35 below exemplifies the graph that was used with the equation of the line used for back-calculating the of sea level ISP value.

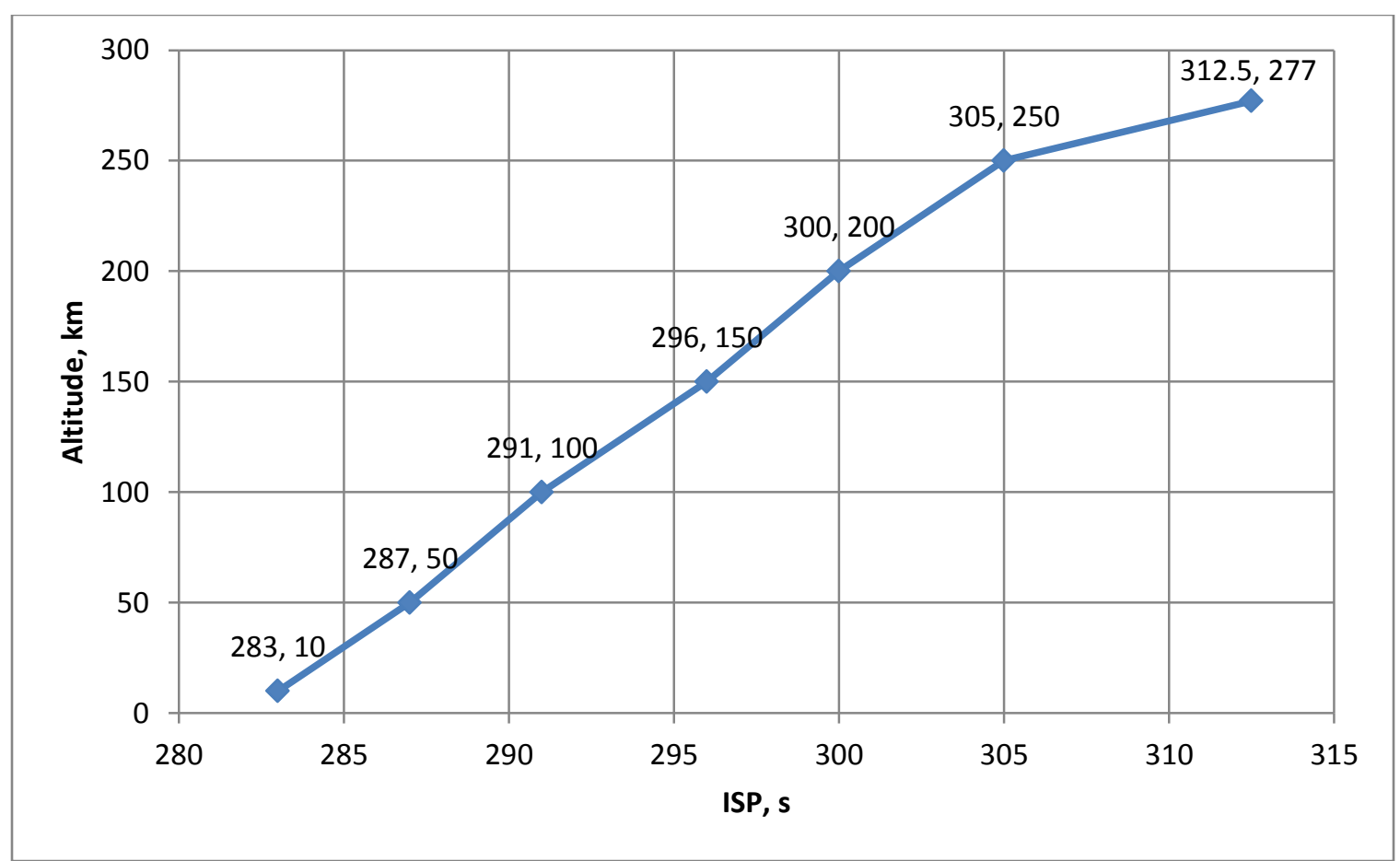

Figure 35 ISP related to the Change in Altitude

The percent differences displayed above will clarify where some of the errors from the geometry creation that will propagate through to the reported solutions. The percent differences calculated were used as error bars for the calculated runs below. Even though this method may not be optimal, it still yielded acceptable results within the scope of this study. More optimal results may be pursued in a future study. 


\section{Force Data for Iteration One}

Table 10 Total Forces and Moments on Each Patch of Wall BC for Iteration One

\begin{tabular}{|c|c|c|c|c|c|c|}
\hline \multicolumn{7}{|c|}{ Stage 1 LOX RP1 Output } \\
\hline $\mathrm{Fx}(\mathrm{N})$ & $\mathrm{Fy}(\mathrm{N})$ & $\mathrm{Fz}(\mathrm{N})$ & $\mathrm{Mx}(\mathrm{N}-\mathrm{m})$ & $\mathrm{My}(\mathrm{N}-\mathrm{m})$ & $\mathrm{Mz}(\mathrm{N}-\mathrm{m})$ & Zone \\
\hline$-1.9254 \mathrm{E}+05$ & $5.1205 \mathrm{E}+05$ & 0 & 0 & 0 & $6.4441 \mathrm{E}+05$ & 1 \\
\hline $2.0823 \mathrm{E}+05$ & $7.0542 \mathrm{E}+05$ & 0 & 0 & 0 & $4.0482 \mathrm{E}+05$ & 2 \\
\hline $5.1742 \mathrm{E}-01$ & $3.0668 \mathrm{E}+06$ & 0 & 0 & 0 & $8.5968 \mathrm{E}+05$ & 3 \\
\hline \multicolumn{7}{|c|}{ Stage 1 LOX LH2 Output } \\
\hline $\mathrm{Fx}(\mathrm{N})$ & $\mathrm{Fy}(\mathrm{N})$ & $\mathrm{Fz}(\mathrm{N})$ & $\mathrm{Mx}(\mathrm{N}-\mathrm{m})$ & $\mathrm{My}(\mathrm{N}-\mathrm{m})$ & $\mathrm{Mz}(\mathrm{N}-\mathrm{m})$ & Zone \\
\hline$-1.6540 \mathrm{E}+05$ & $4.3894 \mathrm{E}+05$ & 0 & 0 & 0 & $5.5064 \mathrm{E}+05$ & 1 \\
\hline $2.1447 \mathrm{E}+05$ & $7.1393 \mathrm{E}+05$ & 0 & 0 & 0 & $4.0801 \mathrm{E}+05$ & 2 \\
\hline $7.5466 \mathrm{E}-01$ & $3.1606 \mathrm{E}+06$ & 0 & 0 & 0 & $8.8615 \mathrm{E}+05$ & 3 \\
\hline \multicolumn{7}{|c|}{ Stage 1 LH2 LF2 Output } \\
\hline $\mathrm{Fx}(\mathrm{N})$ & $\mathrm{Fy}(\mathrm{N})$ & $\mathrm{Fz}(\mathrm{N})$ & $\mathrm{Mx}(\mathrm{N}-\mathrm{m})$ & $\mathrm{My}(\mathrm{N}-\mathrm{m})$ & $\mathrm{Mz}(\mathrm{N}-\mathrm{m})$ & Zone \\
\hline$-1.5487 \mathrm{E}+05$ & $4.1071 \mathrm{E}+05$ & 0 & 0 & 0 & $5.1470 \mathrm{E}+05$ & 1 \\
\hline $2.1689 \mathrm{E}+05$ & $7.1687 \mathrm{E}+05$ & 0 & 0 & 0 & $4.0899 \mathrm{E}+05$ & 2 \\
\hline 7.9038E-01 & $3.1974 \mathrm{E}+06$ & 0 & 0 & 0 & $8.9654 \mathrm{E}+05$ & 3 \\
\hline \multicolumn{7}{|c|}{ Stage 2 LOX RP1 Output } \\
\hline $\mathrm{Fx}(\mathrm{N})$ & $\mathrm{Fy}(\mathrm{N})$ & $\mathrm{Fz}(\mathrm{N})$ & $\mathrm{Mx}(\mathrm{N}-\mathrm{m})$ & $\mathrm{My}(\mathrm{N}-\mathrm{m})$ & $\mathrm{Mz}(\mathrm{N}-\mathrm{m})$ & Zone \\
\hline$-1.1085 \mathrm{E}+05$ & $2.1129 \mathrm{E}+05$ & 0 & 0 & 0 & $2.2199 \mathrm{E}+05$ & 1 \\
\hline $2.4338 \mathrm{E}-01$ & $1.9036 \mathrm{E}+06$ & 0 & 0 & 0 & $3.8343 \mathrm{E}+05$ & 2 \\
\hline $1.7670 \mathrm{E}+05$ & $4.2320 \mathrm{E}+05$ & 0 & 0 & 0 & $1.6833 \mathrm{E}+05$ & 3 \\
\hline \multicolumn{7}{|c|}{ Stage 2 LOX LH2 Output } \\
\hline $\mathrm{Fx}(\mathrm{N})$ & $\mathrm{Fy}(\mathrm{N})$ & $\mathrm{Fz}(\mathrm{N})$ & $\mathrm{Mx}(\mathrm{N}-\mathrm{m})$ & $\mathrm{My}(\mathrm{N}-\mathrm{m})$ & $\mathrm{Mz}(\mathrm{N}-\mathrm{m})$ & Zone \\
\hline$-4.1158 \mathrm{E}+04$ & $7.8420 \mathrm{E}+04$ & 0 & 0 & 0 & $8.1802 \mathrm{E}+04$ & 1 \\
\hline $3.9070 \mathrm{E}-01$ & $9.0663 \mathrm{E}+05$ & 0 & 0 & 0 & $1.8265 \mathrm{E}+05$ & 2 \\
\hline $8.4029 \mathrm{E}+04$ & $1.9803 \mathrm{E}+05$ & 0 & 0 & 0 & $7.8391 \mathrm{E}+04$ & 3 \\
\hline \multicolumn{7}{|c|}{ Stage 2 LH2 LF2 Output } \\
\hline $\mathrm{Fx}(\mathrm{N})$ & $\mathrm{Fy}(\mathrm{N})$ & $\mathrm{Fz}(\mathrm{N})$ & $\mathrm{Mx}(\mathrm{N}-\mathrm{m})$ & $\mathrm{My}(\mathrm{N}-\mathrm{m})$ & $\mathrm{Mz}(\mathrm{N}-\mathrm{m})$ & Zone \\
\hline$-8.3464 \mathrm{E}+04$ & $1.5904 \mathrm{E}+05$ & 0 & 0 & 0 & $1.6555 \mathrm{E}+05$ & 1 \\
\hline $3.8497 \mathrm{E}-01$ & $2.0358 \mathrm{E}+06$ & 0 & 0 & 0 & $4.1017 \mathrm{E}+05$ & 2 \\
\hline $1.8855 \mathrm{E}+05$ & $4.4156 \mathrm{E}+05$ & 0 & 0 & 0 & $1.7446 \mathrm{E}+05$ & 3 \\
\hline
\end{tabular}


Table 11 below displays the derived information from the previous section's summarized output data from the various runs. The sea level thrust was calculated by approximating the curvature of the nozzle wall with a chord. This chord was taken to be the resultant force that the nozzle wall experienced. The resultant force was found and calculated using the $\mathrm{x}$ and $\mathrm{y}$ direction forces as vectors. The values were then converted to a more mainstream unit of measurement for thrust. The ISP values were calculated using the EXCEL calculator that was originally created to generate the contours. The calculated resultant force on the nozzle wall was used as the input to generate the ISP.

Table 11 Calculated Thrust and ISP from CFD Data using Iteration One Inputs

\begin{tabular}{|c|c|c|c|c|c|c|}
\hline Stage & \multicolumn{3}{|c|}{ I } & \multicolumn{3}{c|}{ II } \\
\hline Reactants & LOX/RP1 & LOX/LH2 & LF2/LH2 & LOX/RP1 & LOX/LH2 & LF2/LH2 \\
\hline $\begin{array}{c}\text { Sea Level } \\
\text { Thrust, lbf }\end{array}$ & 153163.96 & 131295.36 & 122851.23 & 67447.71 & 63214.87 & 50768.54 \\
\hline $\begin{array}{c}\text { Sea Level ISP, } \\
\text { s }\end{array}$ & 277.57 & 364.72 & 367.37 & 309.98 & 400.62 & 401.19 \\
\hline
\end{tabular}

Figure 36 and Figure 40 below show the graphs of the thrust to the ISP from iteration one inputs for stage I and II. Both graphs display an inverse relationship between thrust and ISP: for the inputs used, this shows that as the thrust increases, the ISP decreases and vice versa. This is important to note because it is consisted with other known trends regarding ISP and thrust relationships (Huzel \& Huang, p. 12).

Furthermore, the points that represent LOX/H2 and LH2/LF2 are closer together in performance because of their similarity in molecular weights. This indicates the strong influence of molecular weight in both ISP and thrust characteristics. Table 12 displays 
the percent change for comparison with the chart. These percentages were calculated against the published data for the propulsion systems.

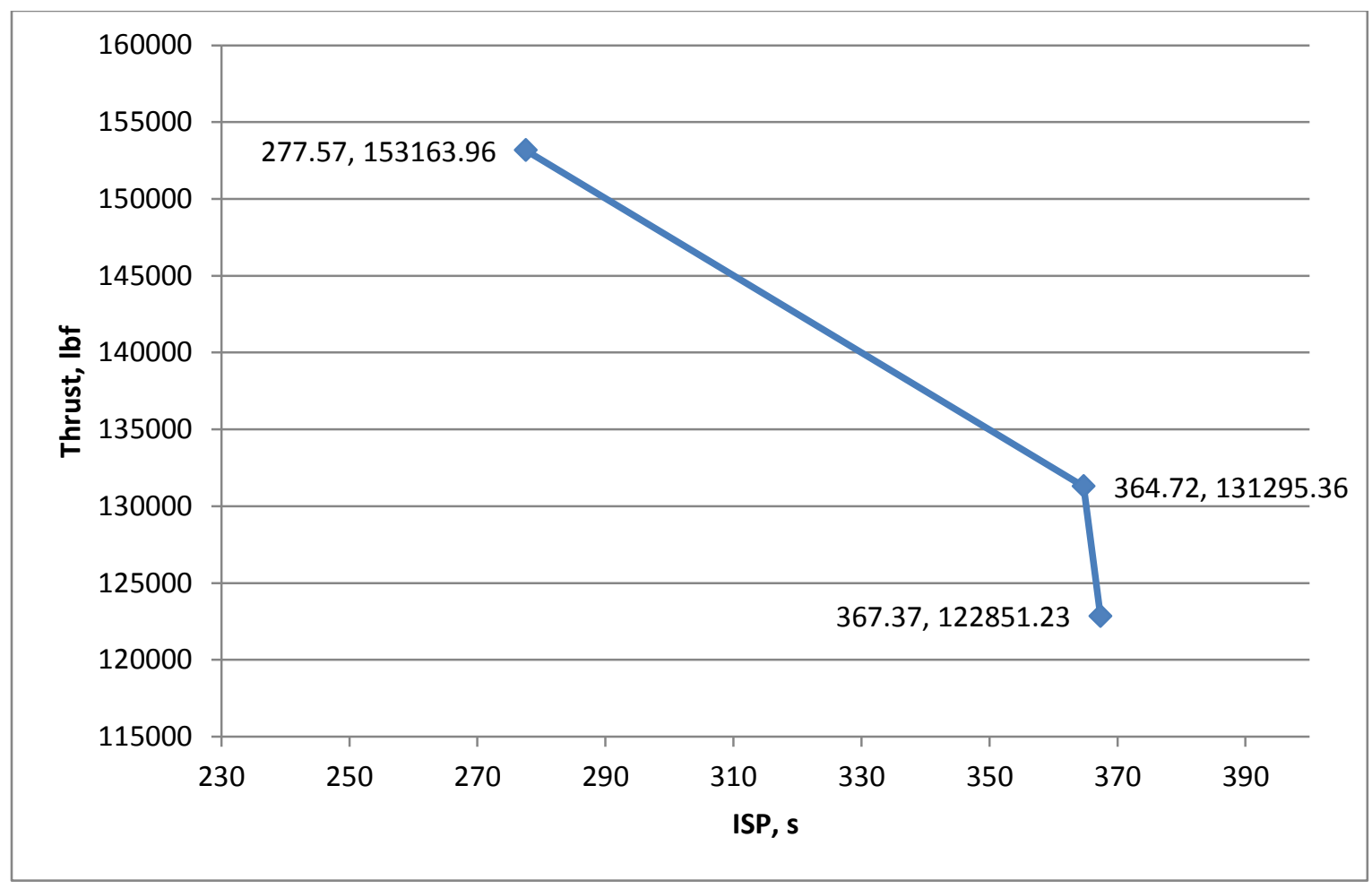

Figure 36 ISP compared to Thrust for Stage I for Iteration One 


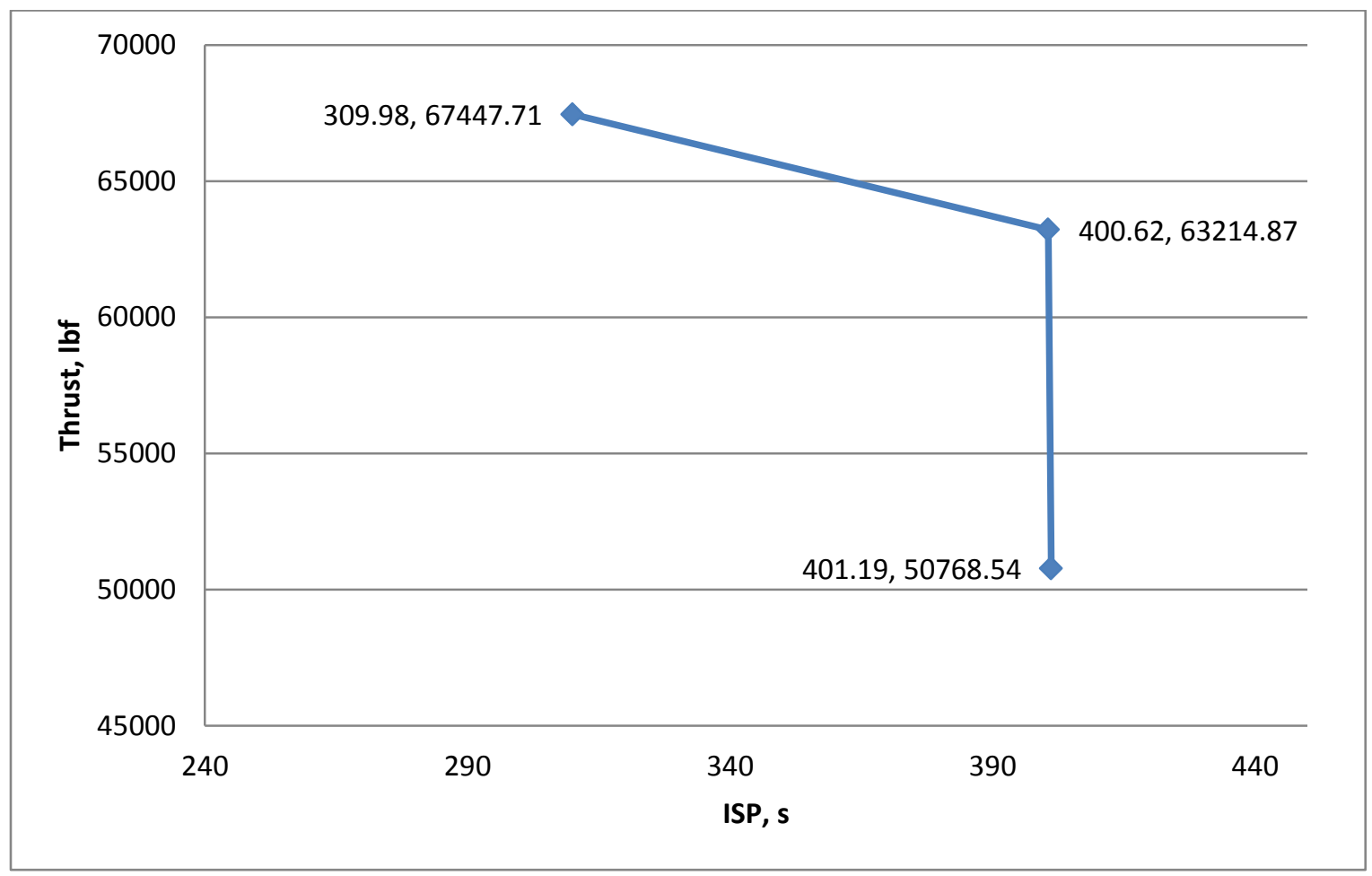

Figure 37 ISP Compared to Thrust for Stage II for Iteration One

Table 12 Percent Difference Comparison with Published Data for Table Above

\begin{tabular}{|c|c|c|c|c|c|c|}
\hline Stage & \multicolumn{3}{|c|}{ I } & \multicolumn{3}{c|}{ II } \\
\hline Reactants & LOX/RP1 & LOX/LH2 & LF2/LH2 & LOX/RP1 & LOX/LH2 & LF2/LH2 \\
\hline $\begin{array}{c}\text { Sea Level Thrust, } \\
\text { lbf }\end{array}$ & $2.11 \%$ & $-12.47 \%$ & $18.09 \%$ & $-15.69 \%$ & $-20.98 \%$ & -36.54 \\
\hline Sea Level ISP, s & $10.19 \%$ & $44.79 \%$ & $45.84 \%$ & $9.53 \%$ & $41.56 \%$ & $41.76 \%$ \\
\hline
\end{tabular}




\section{Force Data for Iteration Two}

Table 13 Total Forces and Moments on Each Patch of Wall BC for Iteration Two

\begin{tabular}{|c|c|c|c|c|c|c|}
\hline \multicolumn{7}{|c|}{ Stage 1 LOX RP1 Output } \\
\hline $\mathrm{Fx}(\mathrm{N})$ & $\mathrm{Fy}(\mathrm{N})$ & $\mathrm{Fz}(\mathrm{N})$ & $\mathrm{Mx}(\mathrm{N}-\mathrm{m})$ & $\mathrm{My}(\mathrm{N}-\mathrm{m})$ & $\mathrm{Mz}(\mathrm{N}-\mathrm{m})$ & Zone \\
\hline$-1.1466 \mathrm{E}+05$ & $3.4734 \mathrm{E}+05$ & 0 & 0 & 0 & $5.1584 \mathrm{E}+05$ & 1 \\
\hline $1.1045 \mathrm{E}+05$ & $3.0385 \mathrm{E}+05$ & 0 & 0 & 0 & $1.6662 \mathrm{E}+05$ & 2 \\
\hline $4.3464 \mathrm{E}+00$ & $3.5649 \mathrm{E}+05$ & 0 & 0 & 0 & $9.9649 \mathrm{E}+04$ & 3 \\
\hline \multicolumn{7}{|c|}{ Stage 1 LOX LH2 Output } \\
\hline $\mathrm{Fx}(\mathrm{N})$ & $\mathrm{Fy}(\mathrm{N})$ & $\mathrm{Fz}(\mathrm{N})$ & $\mathrm{Mx}(\mathrm{N}-\mathrm{m})$ & $\mathrm{My}(\mathrm{N}-\mathrm{m})$ & $\mathrm{Mz}(\mathrm{N}-\mathrm{m})$ & Zone \\
\hline$-1.5843 \mathrm{E}+05$ & $4.2046 \mathrm{E}+05$ & 0 & 0 & 0 & $5.2745 \mathrm{E}+05$ & 1 \\
\hline $2.0544 \mathrm{E}+05$ & $6.8387 \mathrm{E}+05$ & 0 & 0 & 0 & $3.9083 \mathrm{E}+05$ & 2 \\
\hline 7.5469E-01 & $3.0275 \mathrm{E}+06$ & 0 & 0 & 0 & $8.4884 \mathrm{E}+05$ & 3 \\
\hline \multicolumn{7}{|c|}{ Stage 1 LH2 LF2 Output } \\
\hline $\mathrm{Fx}(\mathrm{N})$ & $\mathrm{Fy}(\mathrm{N})$ & $\mathrm{Fz}(\mathrm{N})$ & $\mathrm{Mx}(\mathrm{N}-\mathrm{m})$ & $\mathrm{My}(\mathrm{N}-\mathrm{m})$ & $\mathrm{Mz}(\mathrm{N}-\mathrm{m})$ & Zone \\
\hline$-2.1139 \mathrm{E}+05$ & $5.6218 \mathrm{E}+05$ & 0 & 0 & 0 & $7.0750 \mathrm{E}+05$ & 1 \\
\hline $2.2861 \mathrm{E}+05$ & $7.7447 \mathrm{E}+05$ & 0 & 0 & 0 & $4.4445 \mathrm{E}+05$ & 2 \\
\hline 7.5034E-01 & $3.3671 \mathrm{E}+06$ & 0 & 0 & 0 & $9.4384 \mathrm{E}+05$ & 3 \\
\hline \multicolumn{7}{|c|}{ Stage 2 LOX RP1 Output } \\
\hline $\mathrm{Fx}(\mathrm{N})$ & $\mathrm{Fy}(\mathrm{N})$ & $\mathrm{Fz}(\mathrm{N})$ & $\mathrm{Mx}(\mathrm{N}-\mathrm{m})$ & $\mathrm{My}(\mathrm{N}-\mathrm{m})$ & $\mathrm{Mz}(\mathrm{N}-\mathrm{m})$ & Zone \\
\hline$-1.0808 \mathrm{E}+05$ & $2.0601 \mathrm{E}+05$ & 0 & 0 & 0 & $2.1645 \mathrm{E}+05$ & 1 \\
\hline $2.4602 \mathrm{E}-01$ & $1.8560 \mathrm{E}+06$ & 0 & 0 & 0 & $3.7385 \mathrm{E}+05$ & 2 \\
\hline $1.7228 \mathrm{E}+05$ & $4.1263 \mathrm{E}+05$ & 0 & 0 & 0 & $1.6413 \mathrm{E}+05$ & 3 \\
\hline \multicolumn{7}{|c|}{ Stage 2 LOX LH2 Output } \\
\hline $\mathrm{Fx}(\mathrm{N})$ & $\mathrm{Fy}(\mathrm{N})$ & $\mathrm{Fz}(\mathrm{N})$ & $\mathrm{Mx}(\mathrm{N}-\mathrm{m})$ & $\mathrm{My}(\mathrm{N}-\mathrm{m})$ & $\mathrm{Mz}(\mathrm{N}-\mathrm{m})$ & Zone \\
\hline$-8.8849 \mathrm{E}+04$ & $1.6927 \mathrm{E}+05$ & 0 & 0 & 0 & $1.7657 \mathrm{E}+05$ & 1 \\
\hline $3.6880 \mathrm{E}-01$ & $1.9571 \mathrm{E}+06$ & 0 & 0 & 0 & $3.9428 \mathrm{E}+05$ & 2 \\
\hline $1.8139 \mathrm{E}+05$ & $4.2746 \mathrm{E}+05$ & 0 & 0 & 0 & $1.6921 \mathrm{E}+05$ & 3 \\
\hline \multicolumn{7}{|c|}{ Stage 2 LH2 LF2 Output } \\
\hline $\mathrm{Fx}(\mathrm{N})$ & $\mathrm{Fy}(\mathrm{N})$ & $\mathrm{Fz}(\mathrm{N})$ & $\mathrm{Mx}(\mathrm{N}-\mathrm{m})$ & $\mathrm{My}(\mathrm{N}-\mathrm{m})$ & $\mathrm{Mz}(\mathrm{N}-\mathrm{m})$ & Zone \\
\hline$-8.1961 \mathrm{E}+04$ & $1.5618 \mathrm{E}+05$ & 0 & 0 & 0 & $1.6257 \mathrm{E}+05$ & 1 \\
\hline $3.8570 \mathrm{E}-01$ & $1.9992 \mathrm{E}+06$ & 0 & 0 & 0 & $4.0278 \mathrm{E}+05$ & 2 \\
\hline $1.8516 \mathrm{E}+05$ & $4.3361 \mathrm{E}+05$ & 0 & 0 & 0 & $1.7132 \mathrm{E}+05$ & 3 \\
\hline
\end{tabular}


Table 14 below displays the derived information from the previous section in similar fashion as iteration one, above. First glances reveal the vast differences in the force data, indicating right away the effect of increasing the pressure on the systems compared to iteration one. Another observation includes the way in which some reactants actually perform better under higher pressure than the others in the same iteration, and also in comparison to the previous iteration. Specifically, LF2/LH2 have increased ISP values and thrust in the second iteration.

\section{Table 14 Calculated Thrust and ISP from CFD Data using Iteration Two Inputs}

\begin{tabular}{|c|c|c|c|c|c|c|}
\hline Stage & \multicolumn{3}{|c|}{ I } & \multicolumn{3}{c|}{ II } \\
\hline Reactants & LOX/RP1 & LOX/LH2 & LF2/LH2 & LOX/RP1 & LOX/LH2 & LF2/LH2 \\
\hline $\begin{array}{c}\text { Sea Level } \\
\text { Thrust, lbf }\end{array}$ & 103896 & 125767.64 & 168158.80 & 65762.24 & 54034.14 & 59855.57 \\
\hline Sea Level ISP, s & 292.26 & 380.77 & 382.46 & 318.02 & 408.61 & 408.43 \\
\hline
\end{tabular}

Figure 38 and Figure 39 below show the graphs of the thrust to the ISP from iteration two inputs for stage I and II. The stage I graph displays a trend that goes upward with increasing ISP and thrust moving from one reactant to the next. For the inputs used, this shows that as the thrust increases, the ISP also increases. This is an important discovery as it shows a direct benefit of increasing the pressure in the chamber. The Stage II graph displays a trend opposite to stage I, which is interesting because it suggests that for this geometry, the increased pressure is more beneficial for the original reactant mixture than for any of the new ones suggested. This indicates that pressure increase still plays a large role in the improvement of the propulsion system, despite the 
geometrical variation; however, the extent of the improvement is only optimal for the original mixture. The table below was created as a convenient way to quickly see the percent change from the original published data on the stages.

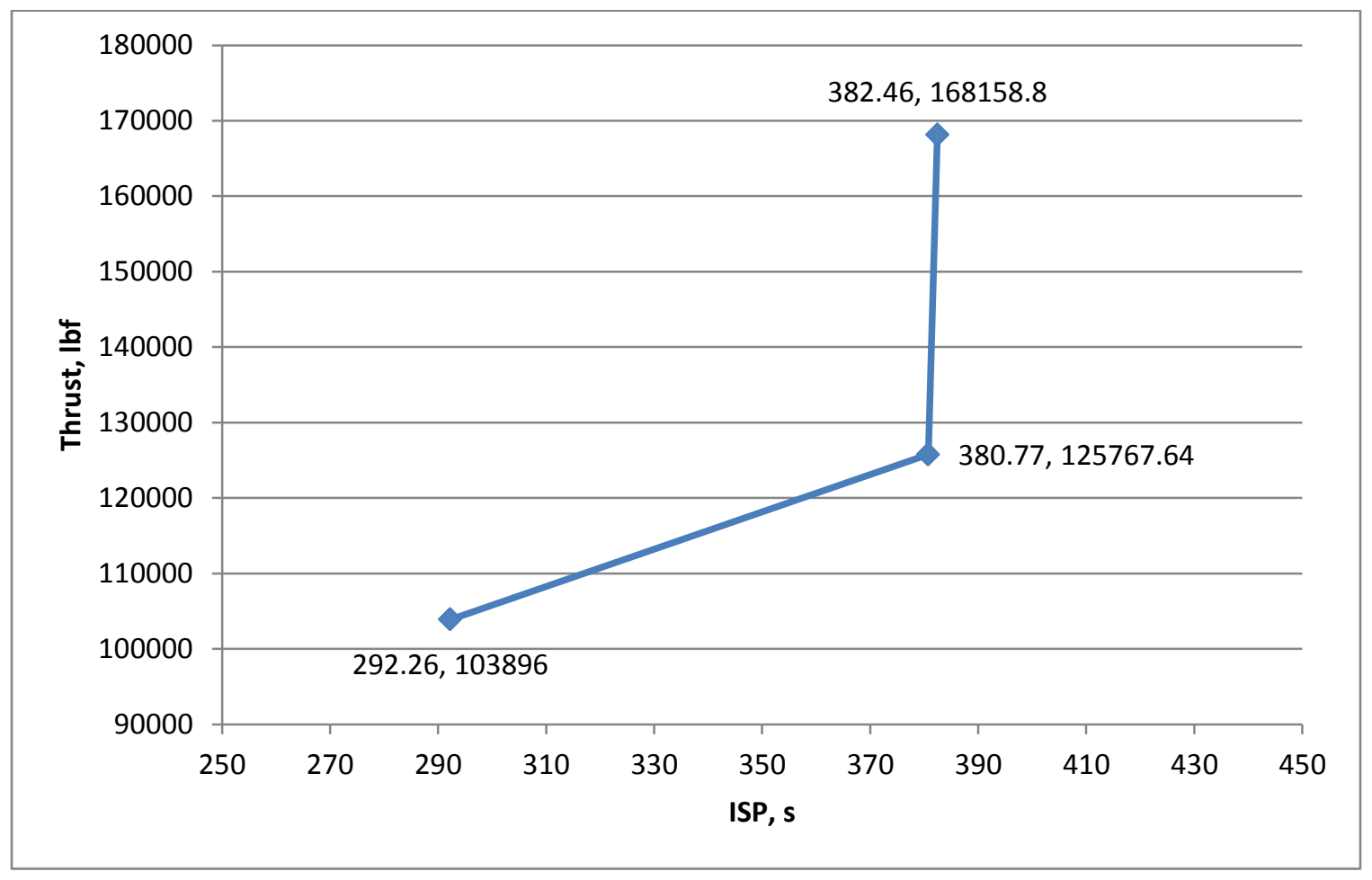

Figure 38 ISP Compared to Thrust for Stage I Iteration Two 


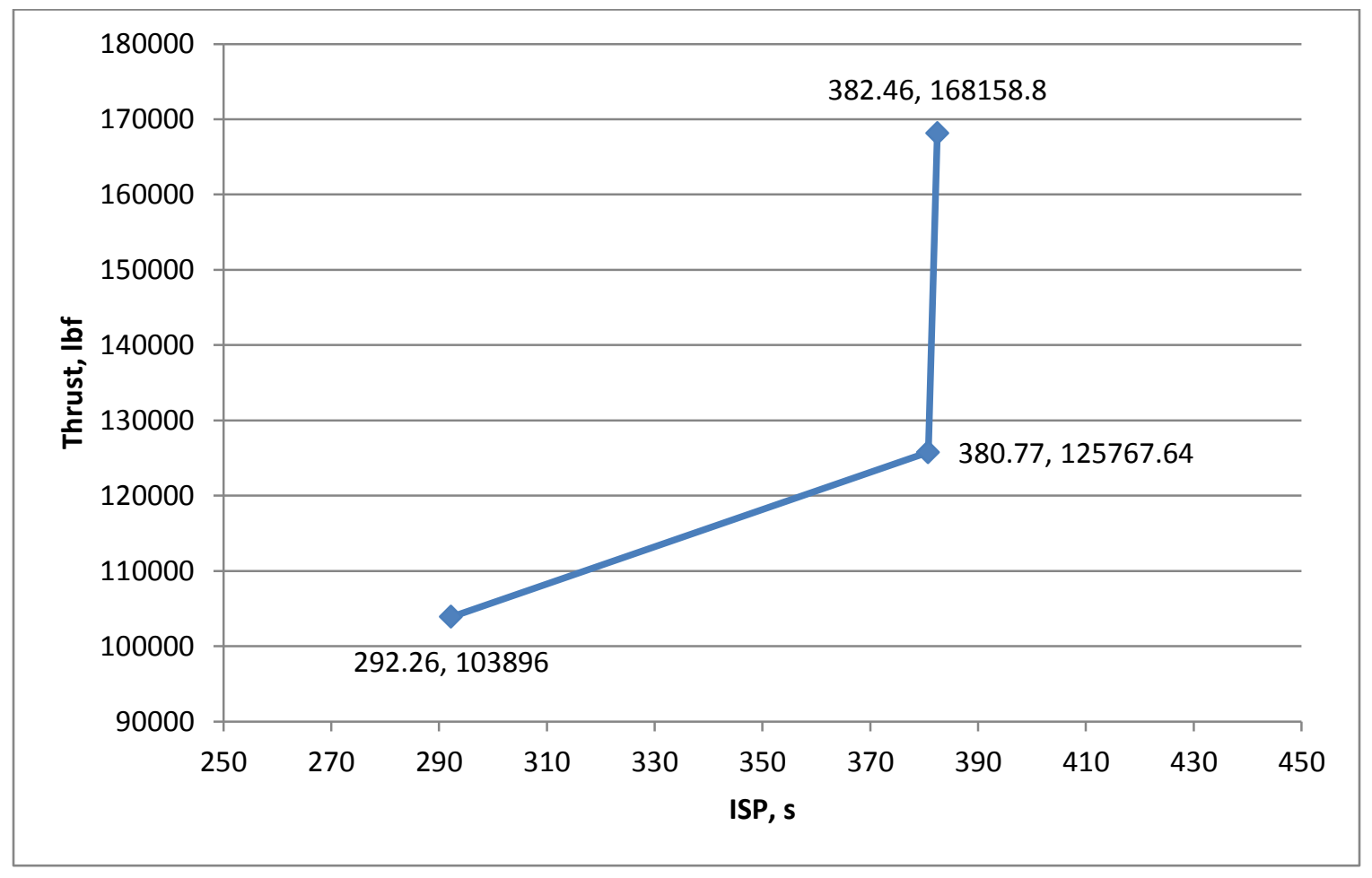

Figure 39 ISP Compared to Thrust for Stage I Iteration Two

Table 15Percent Difference Comparison with Published Data for Table above

\begin{tabular}{|c|c|c|c|c|c|c|}
\hline Stage & \multicolumn{3}{|c|}{ I } & \multicolumn{3}{c|}{ II } \\
\hline Reactants & LOX/RP1 & LOX/LH2 & LF2/LH2 & LOX/RP1 & LOX/LH2 & LF2/LH2 \\
\hline $\begin{array}{c}\text { Sea Level Thrust, } \\
\text { lbf }\end{array}$ & $-30.74 \%$ & $-16.15 \%$ & $12.11 \%$ & $-17.79 \%$ & $-32.46 \%$ & $-25.18 \%$ \\
\hline Sea Level ISP, s & $16.02 \%$ & $51.16 \%$ & $51.83 \%$ & $12.37 \%$ & $44.39 \%$ & $62.14 \%$ \\
\hline
\end{tabular}




\section{Data from CFD - VIEW Iteration One}

Figure 43 displays the Mach zones output for this run. The red line displays the point at which the Mach value equals one for this run and all subsequent runs. The location of the Mach one line is inside of the throat region, meaning that the flow is choked, increasing the likelihood that the contour accurately represents the original. The Mach values of one for all the remaining Stage I outputs are also located in the throat region. As for the Stage II Mach zones, the Mach one line creeps forward. This may be due to the fact that the contour is not optimized for the propellant combination being used, proof of which is outside the scope of this study.

The velocity field figures below have small points near the connection point between the throat and the chamber. This point has a near-zero velocity. This is not

normal and is due to the curvature at that point. It is possible that the geometry may have not been as fine as ideal for this zone; this may be further explored in a future study. None of the other figures show anything out of the ordinary. 
Stage 1 LOX RP1 Output.

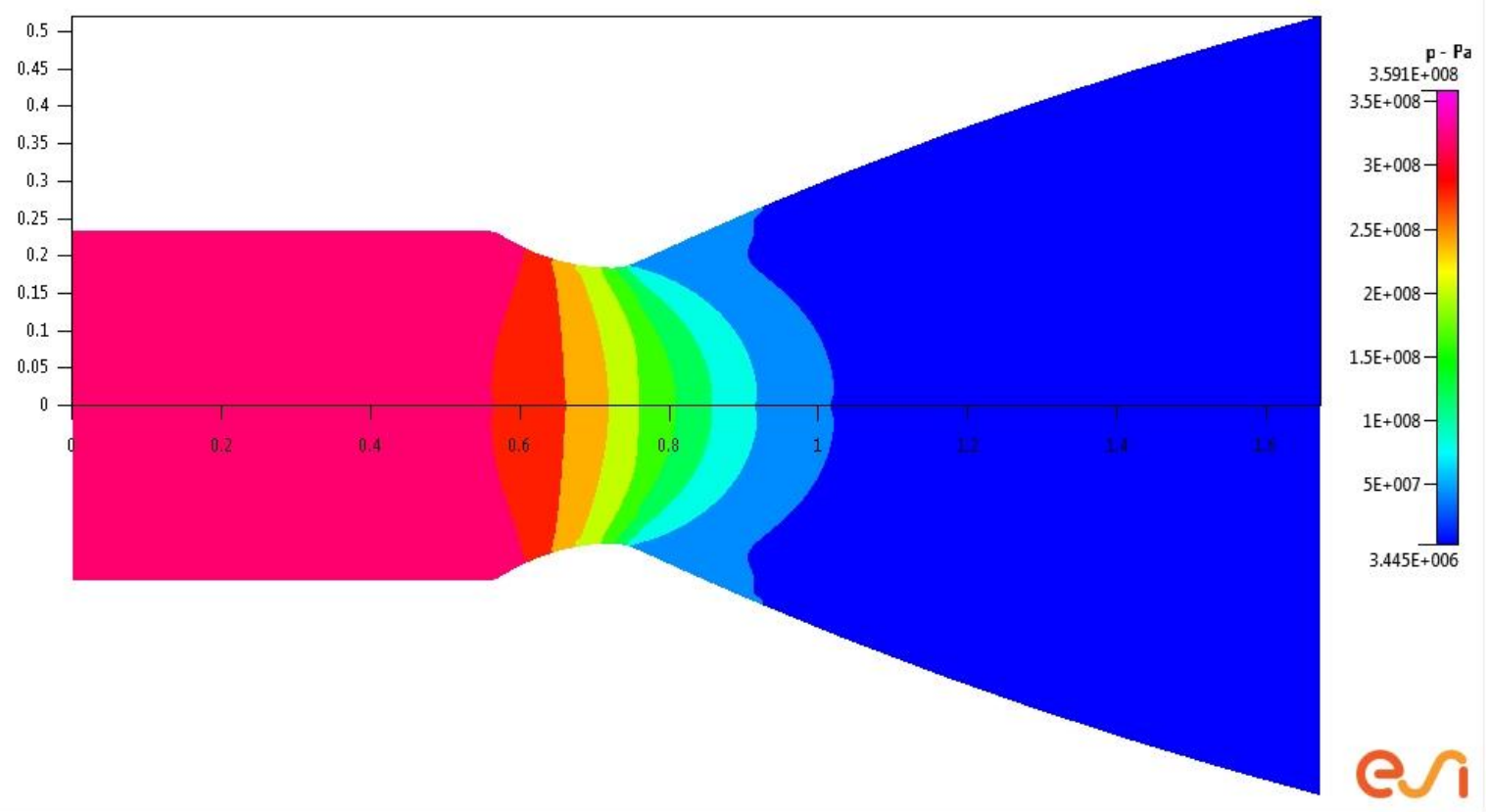

Figure 40 Pressure Map out for Stage 1 LOX/RP1

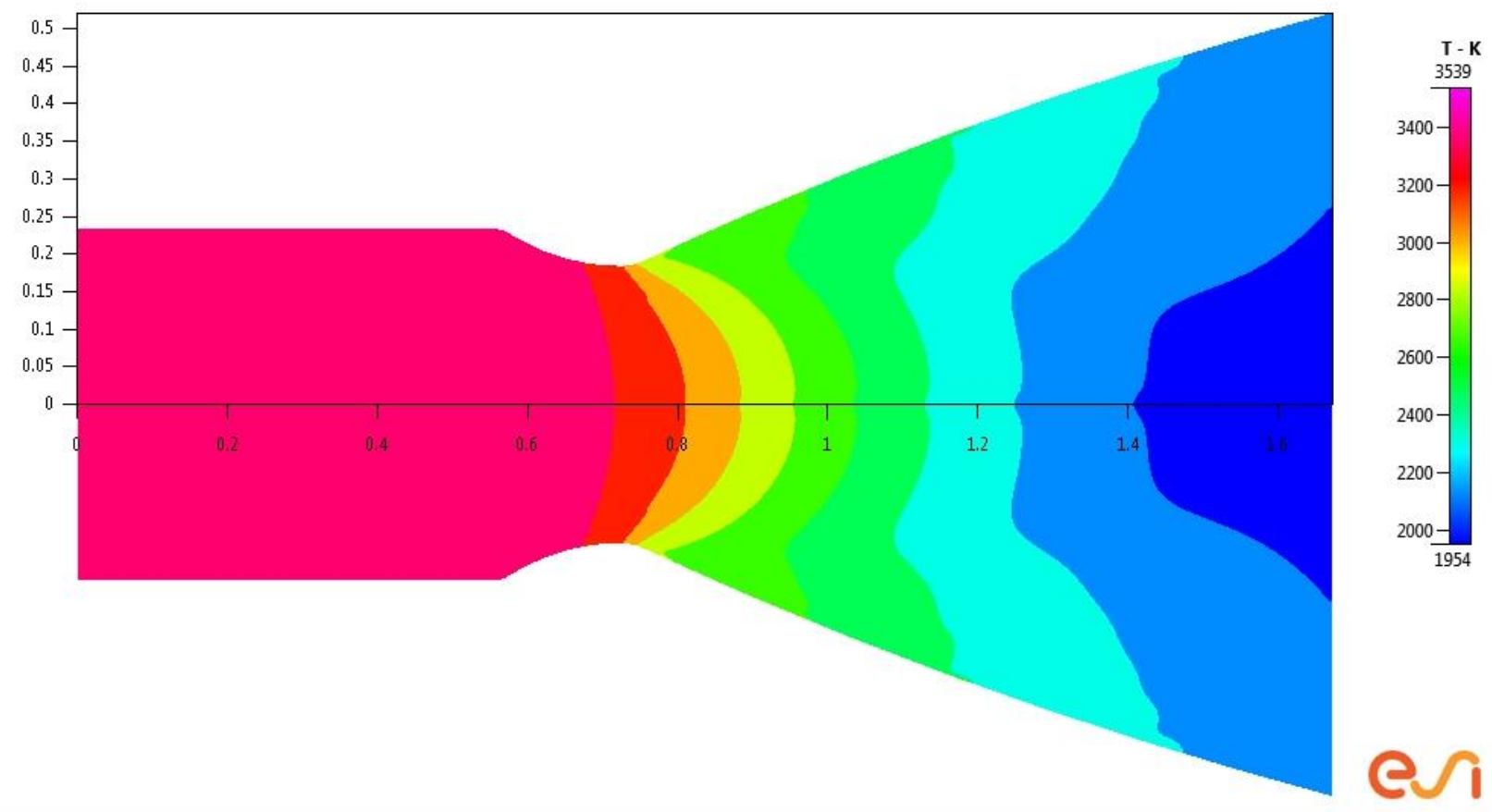

Figure 41 Temperature Map Output for Stage 1 LOX/RP1 


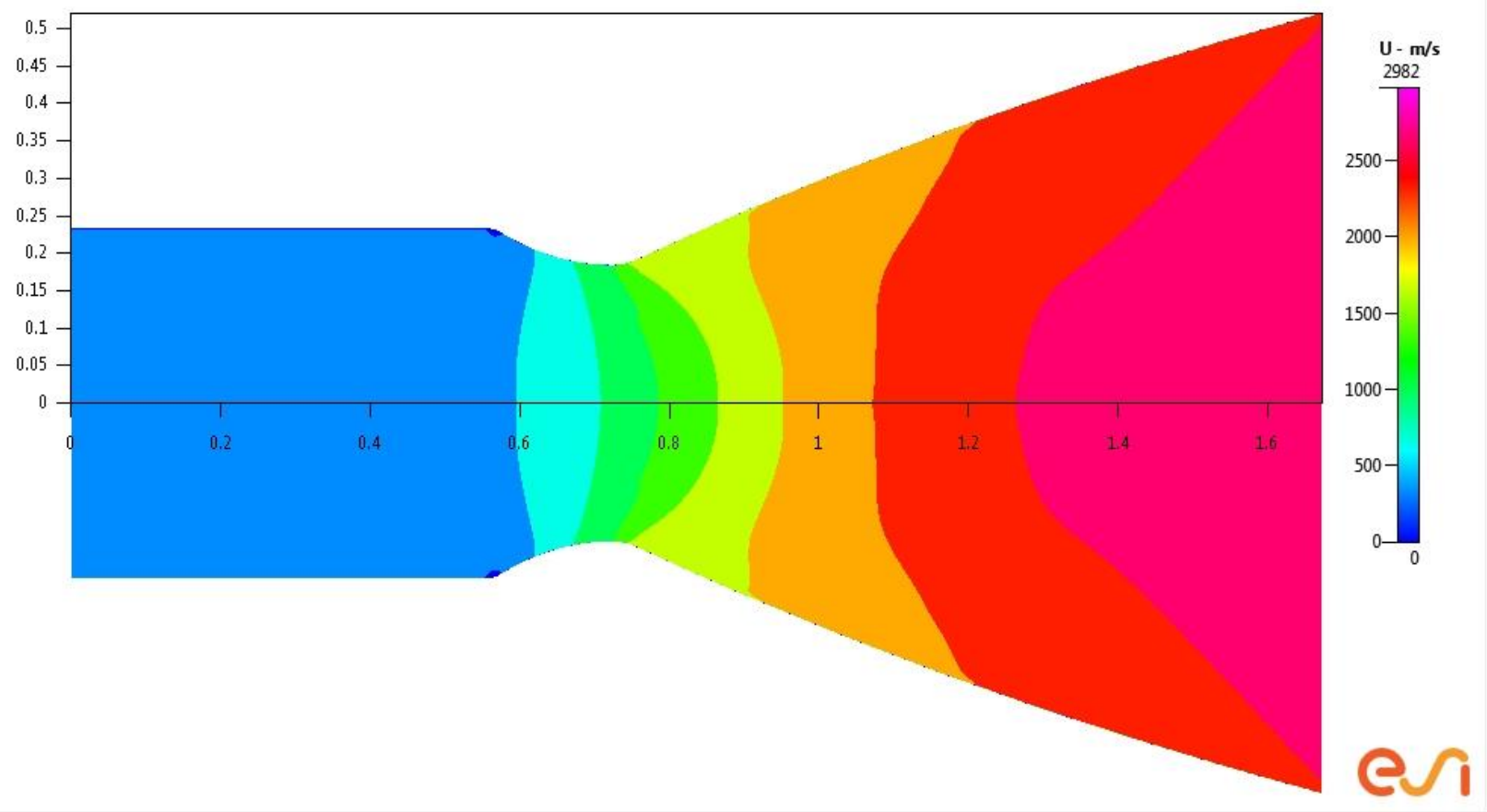

Figure 42 X - Velocity Field for Stage 1 LOX/RP1

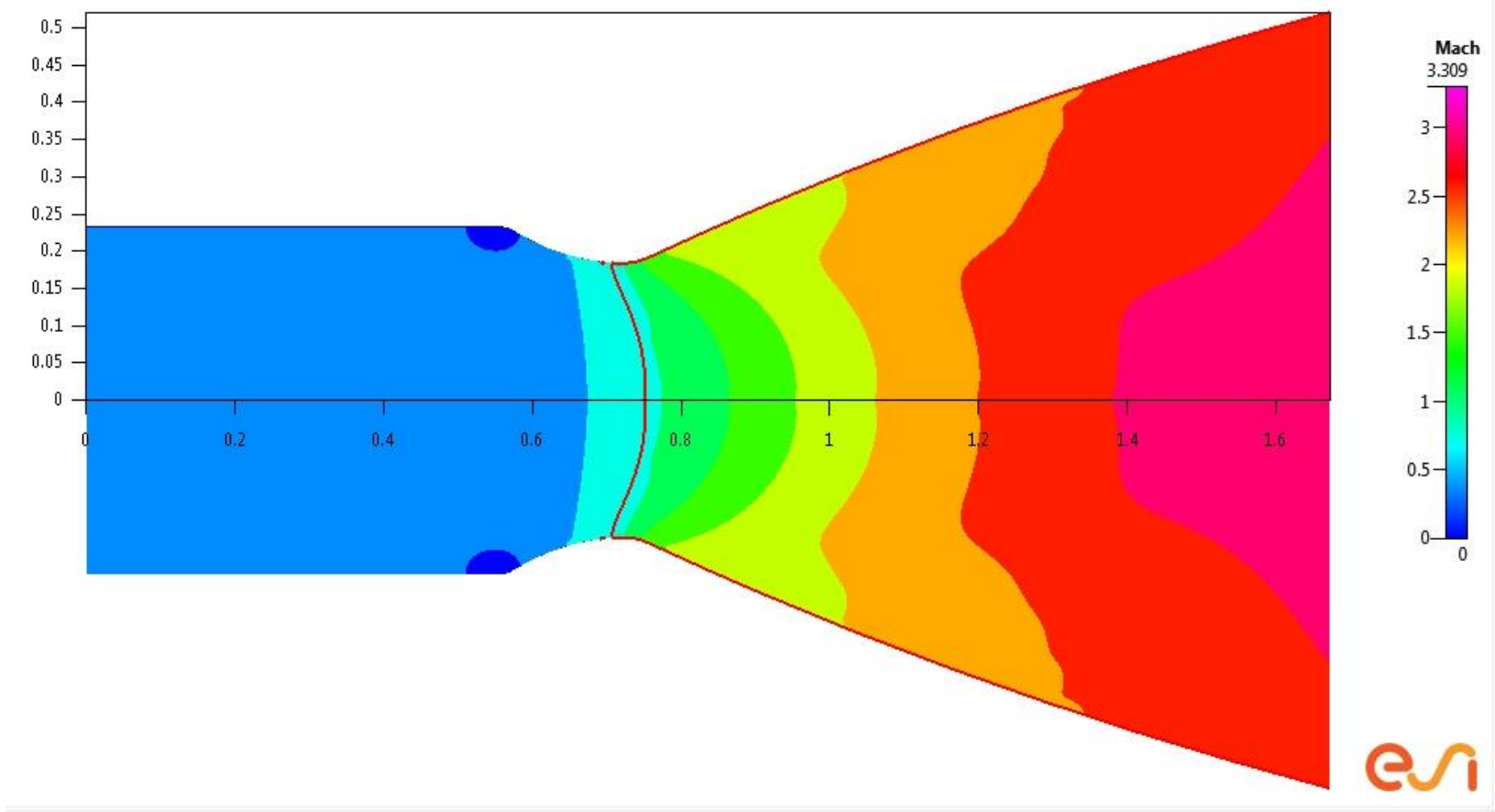

Figure 43 Mach Zones Output for Stage 1 LOX/RP1 
Stage 1 LOX LH2 Output.

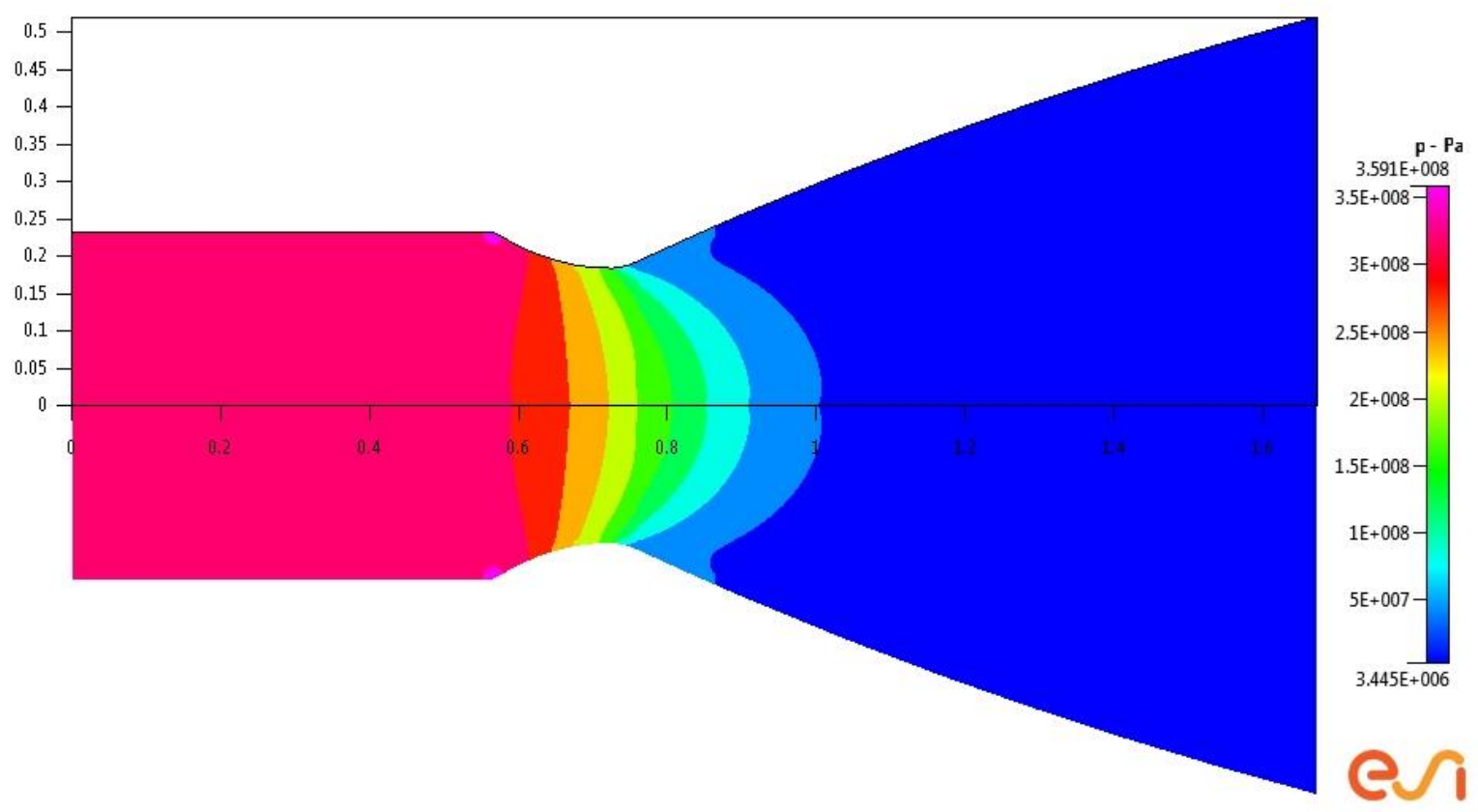

Figure 44 Pressure Map Output for Stage 1 LOX/LH2

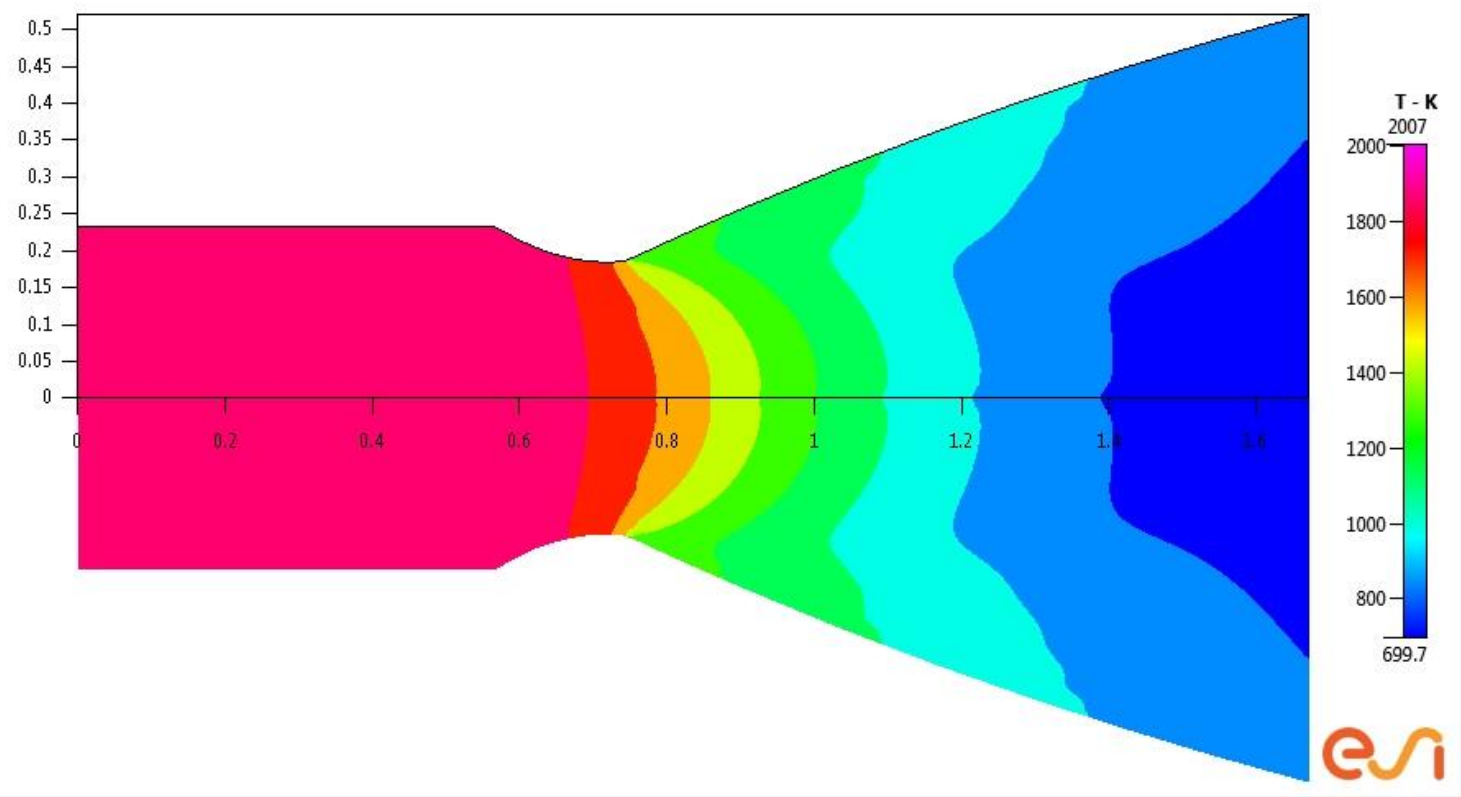

Figure 45 Temperature Map Output for Stage 1 LOX/LH2 


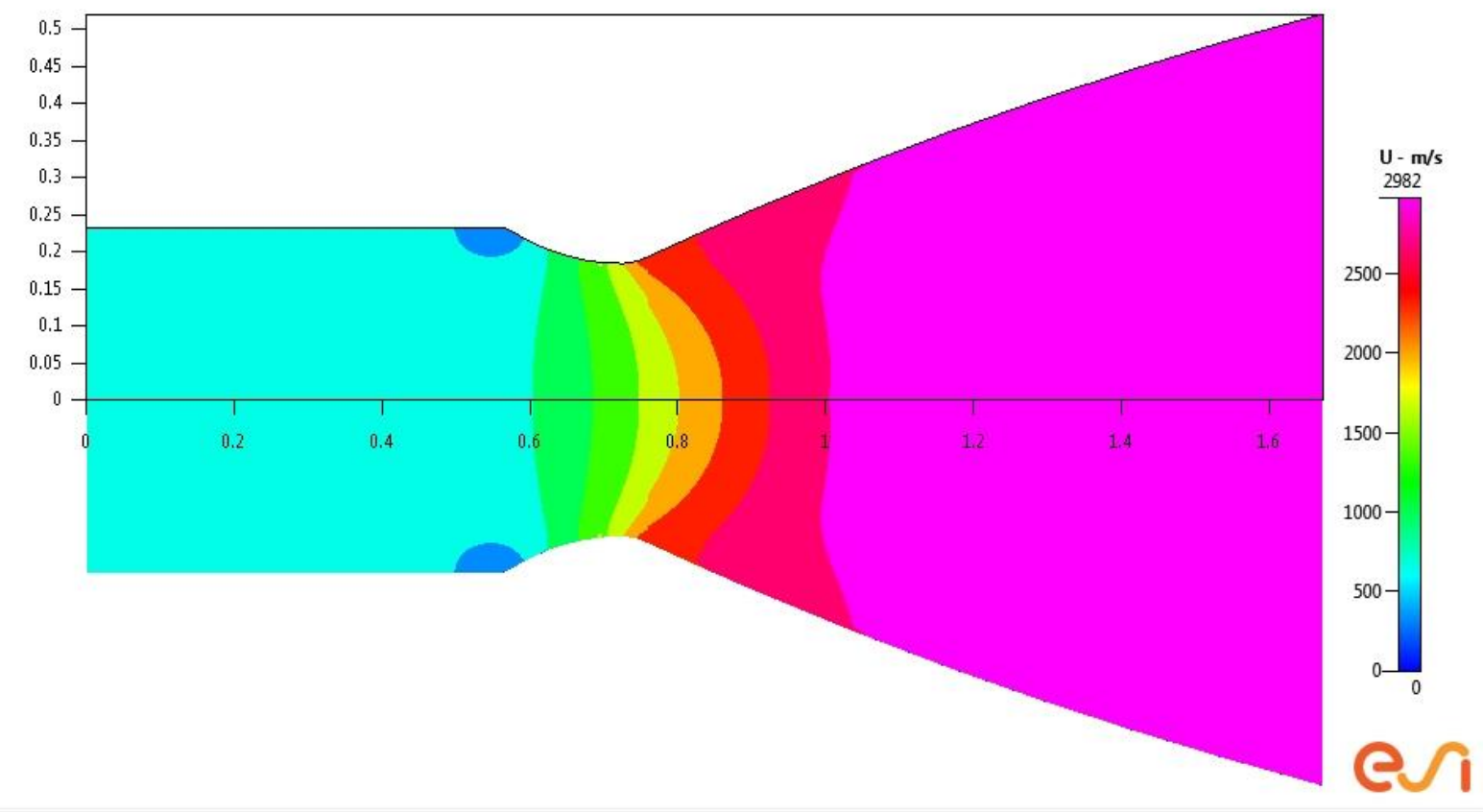

Figure 46 X - Velocity Field for Stage 1 LOX/LH2

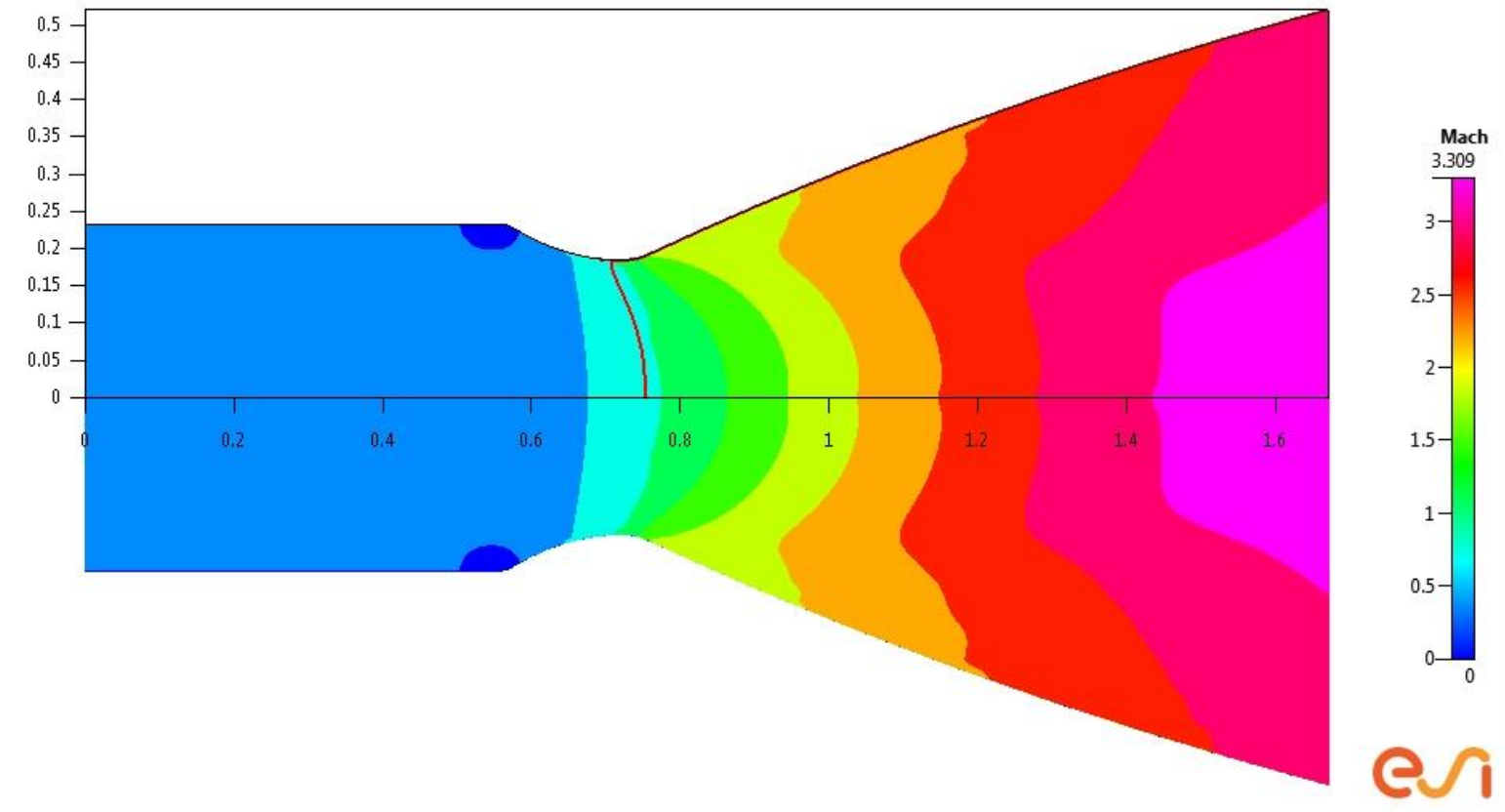

Figure 47 Mach Zone Output for Stage 1 LOX/LH2 
Stage 1 LH2 LF2 Output.

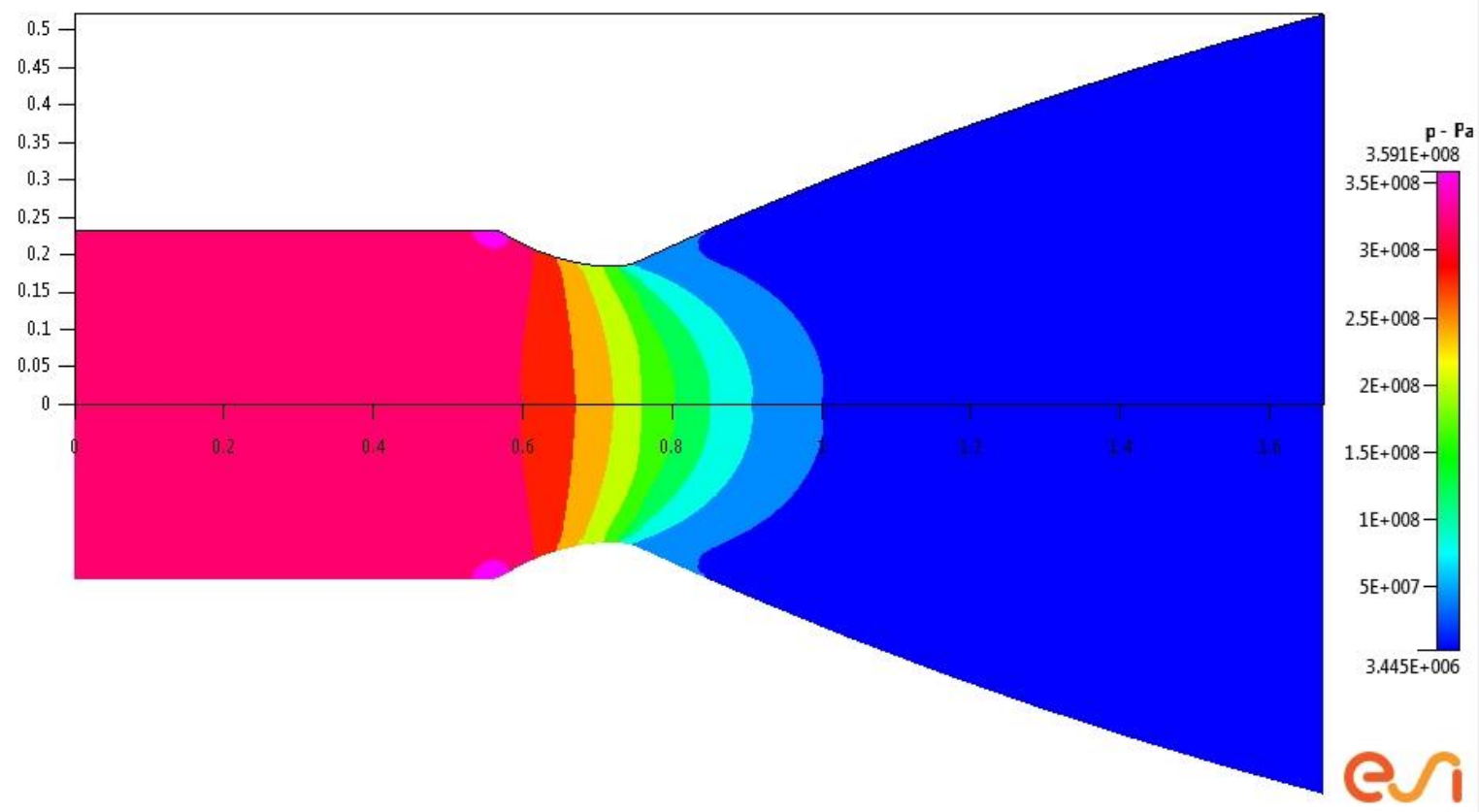

Figure 48 Pressure Map for Stage 1 LH2/LF2

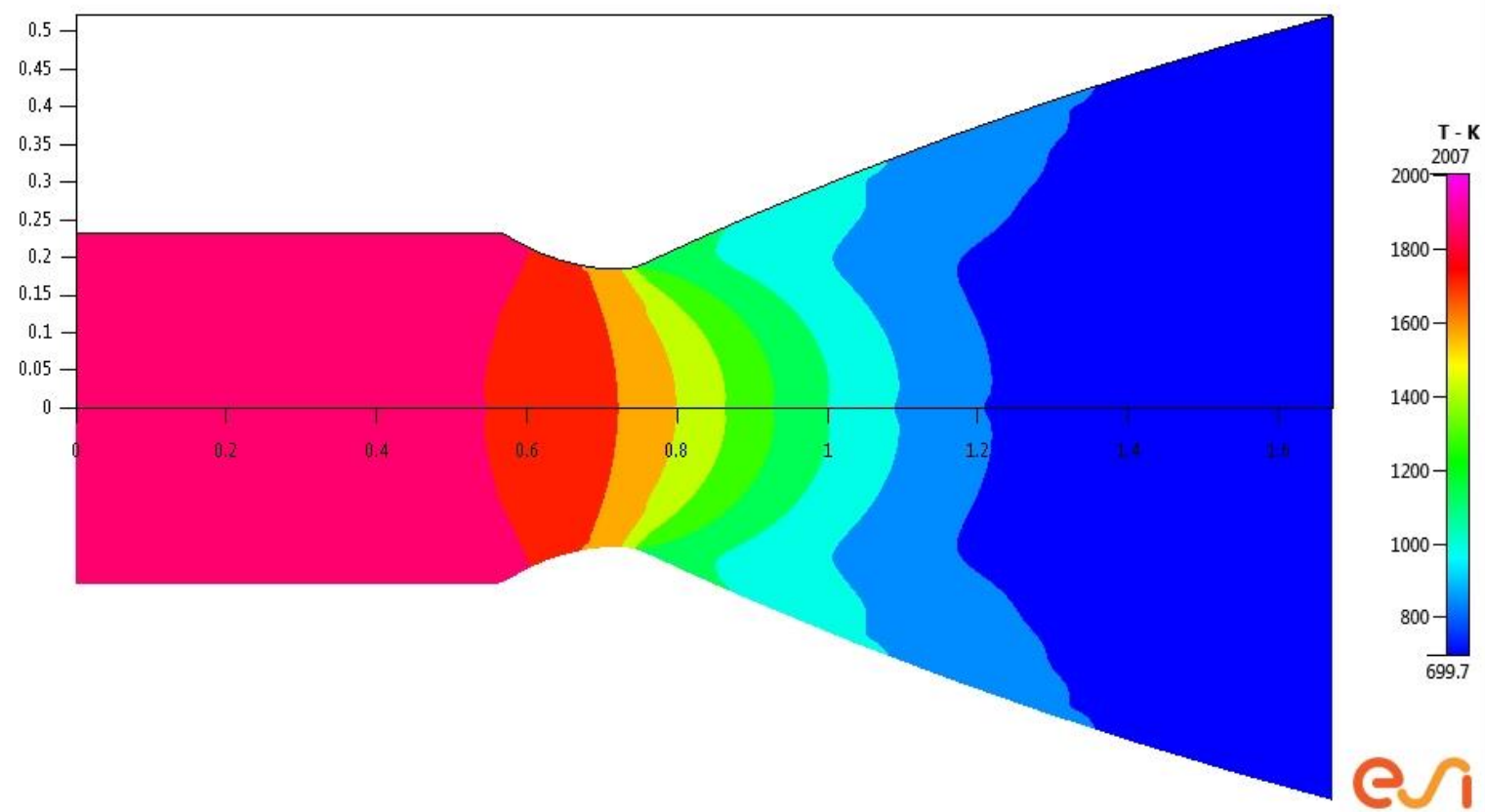

Figure 49 Temperature Map for Stage 1 LH2/LF2 


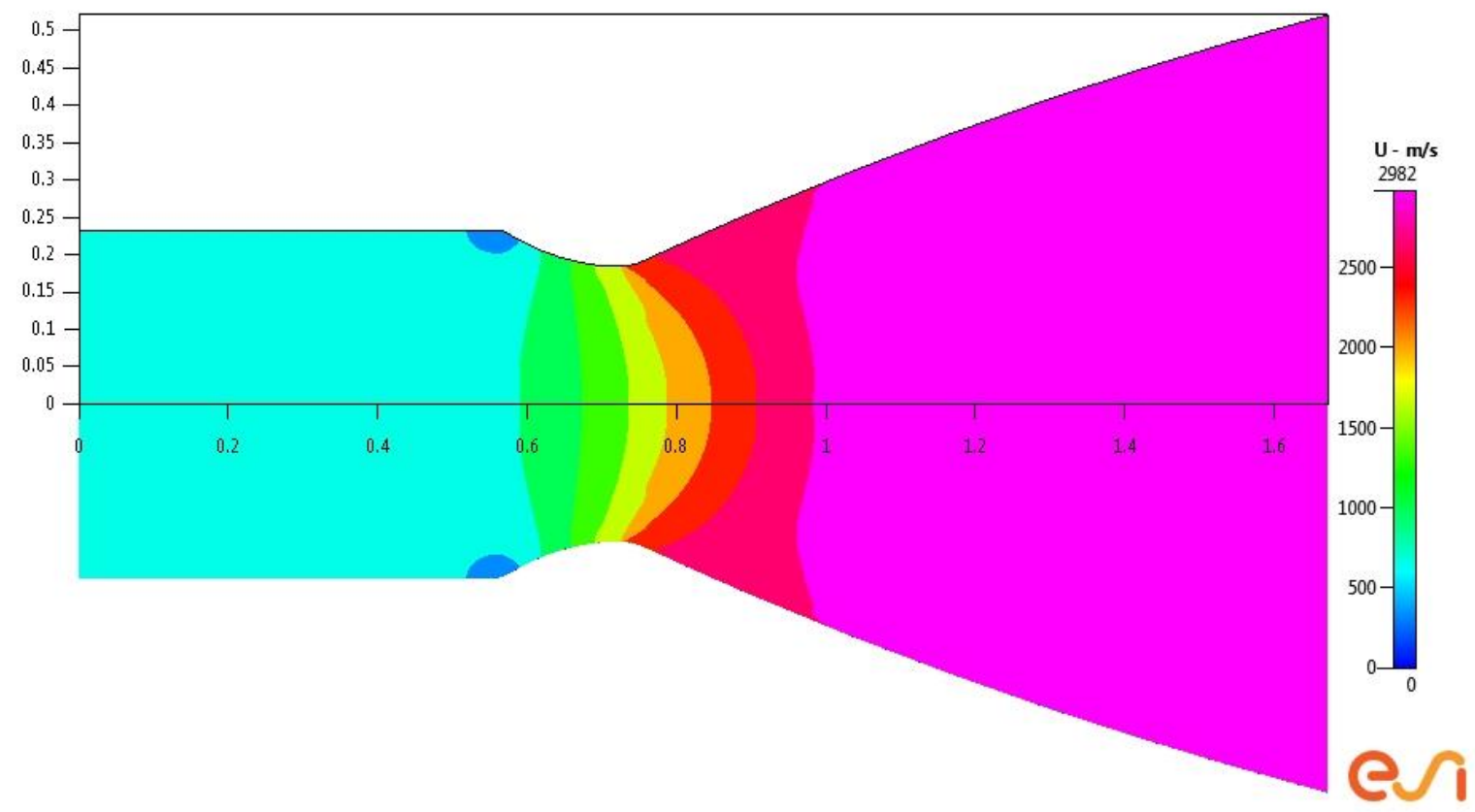

Figure 50 X - Velocity Field for Stage 1 LH2/LF2

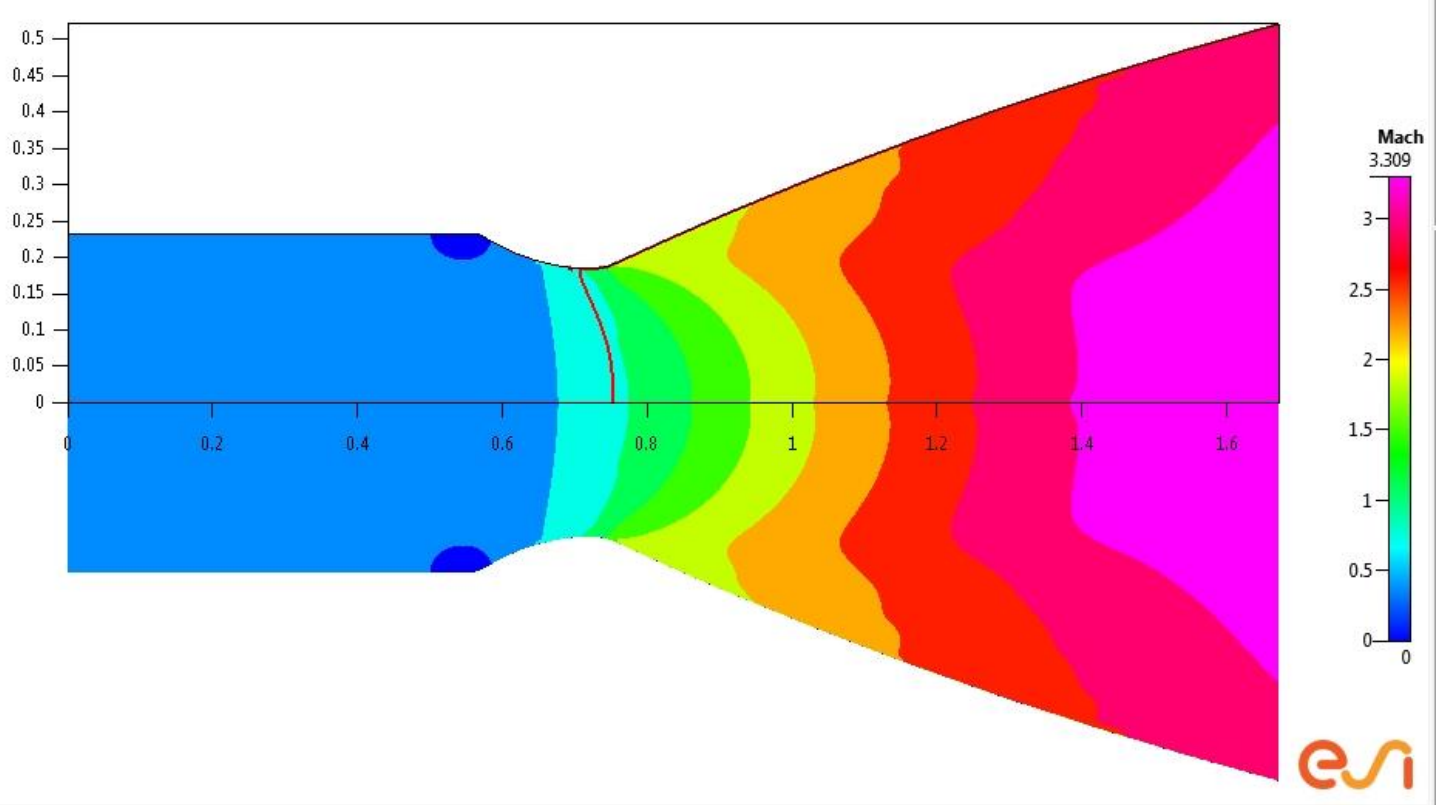

Figure 51 Mach Zones Output for Stage 1 LH2/LF2 
Stage 2 LOX RP1 Outputs.

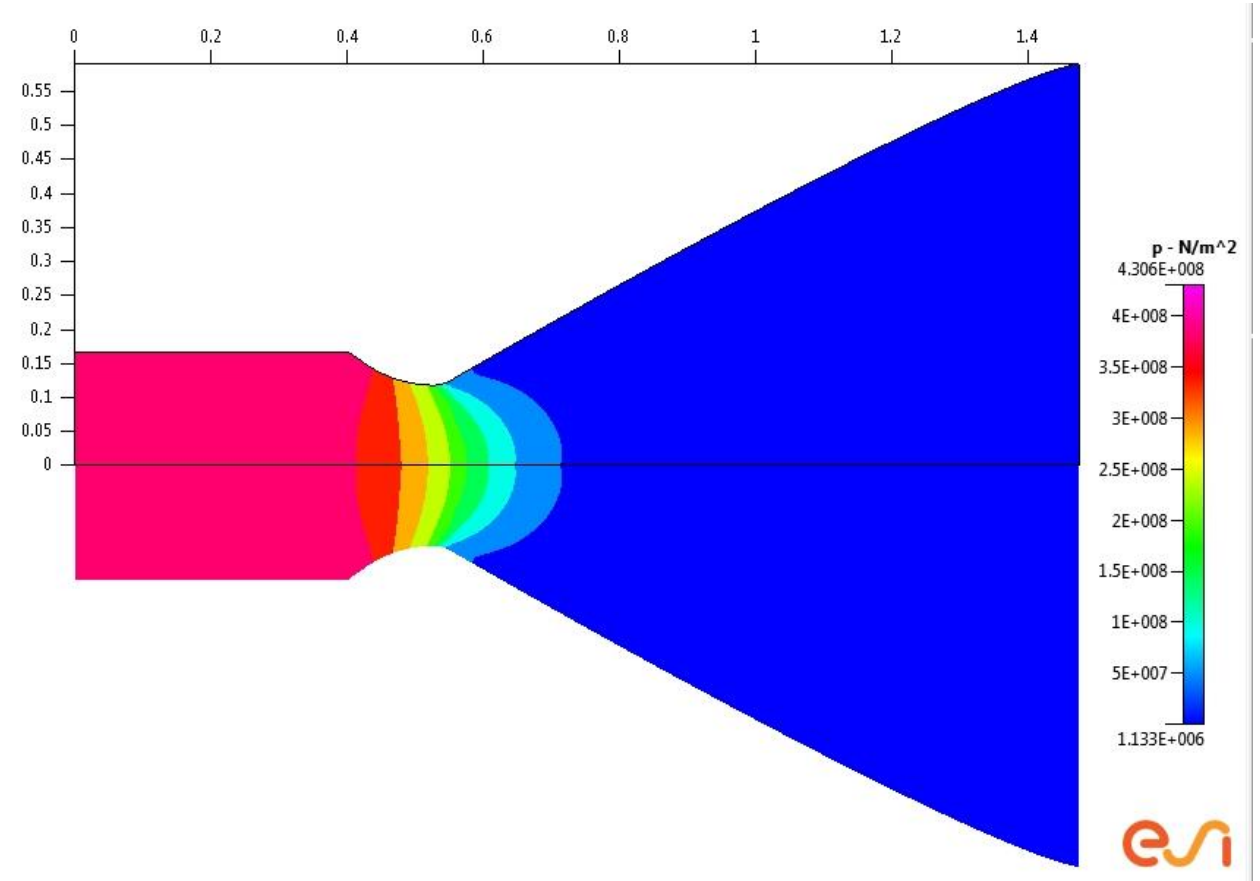

Figure 52 Pressure Map Output Stage 1 LOX/RP1

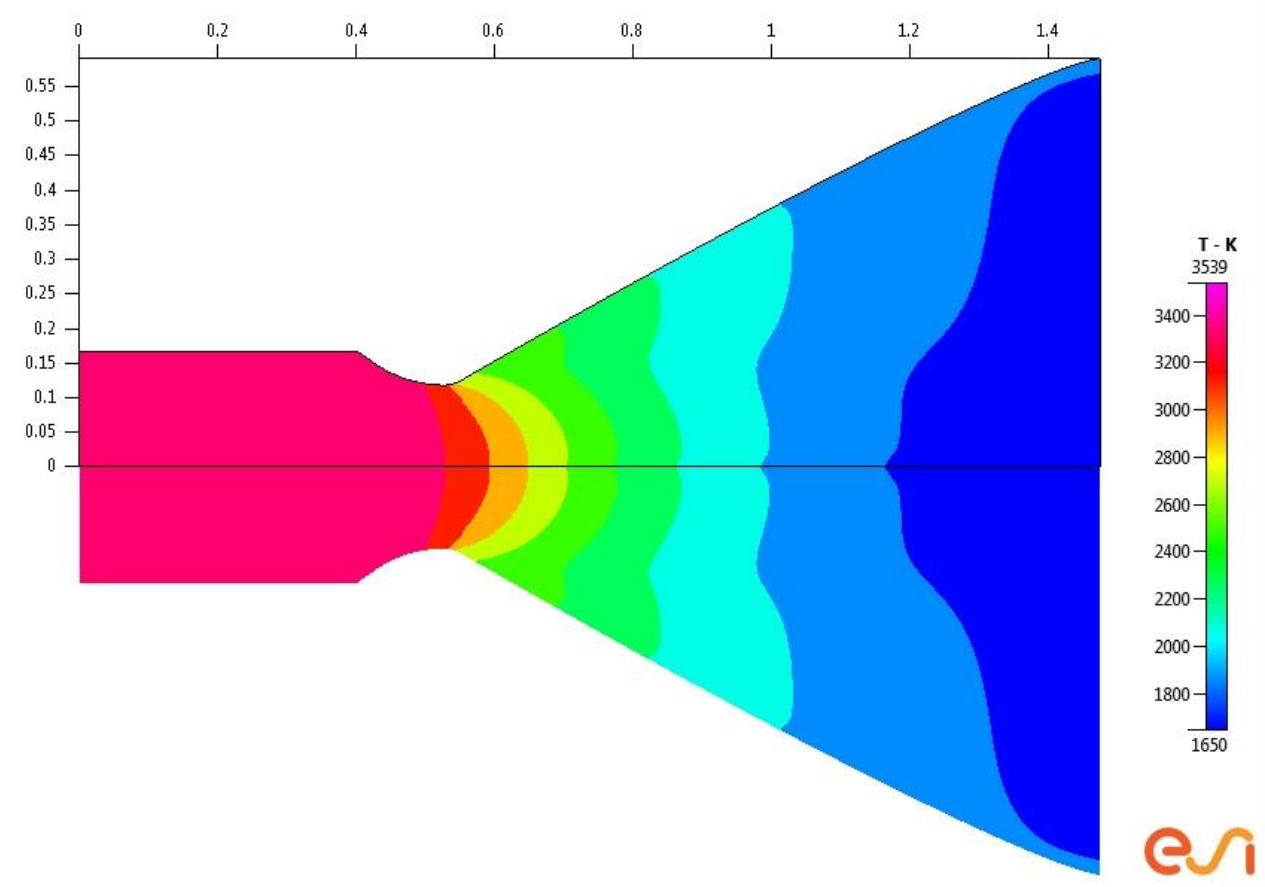

Figure 53 Temperature Map Output for Stage 1 LOX/RP1 


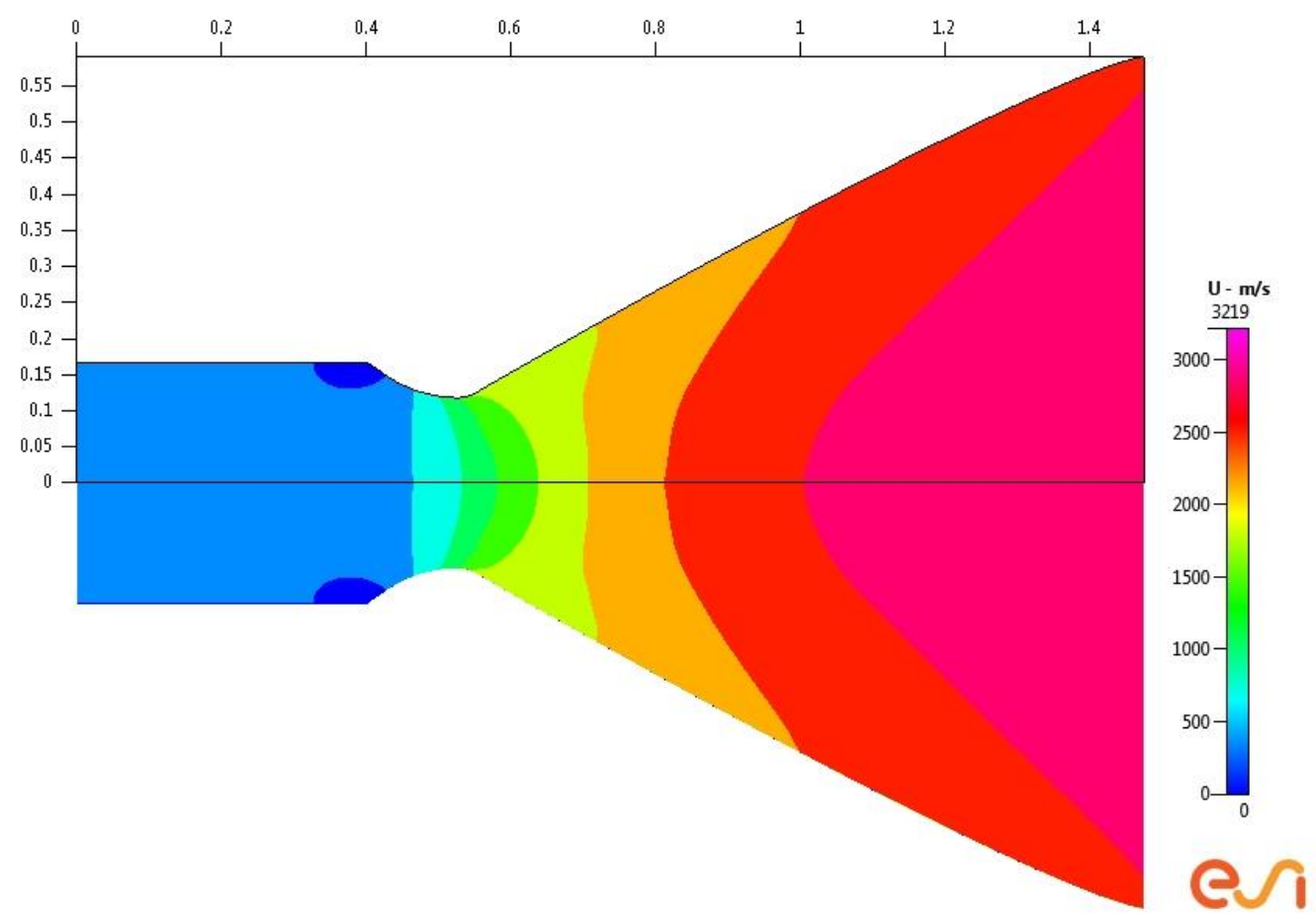

Figure 54 X - Direction Velocity Field for Stage 1 LOX/RP1

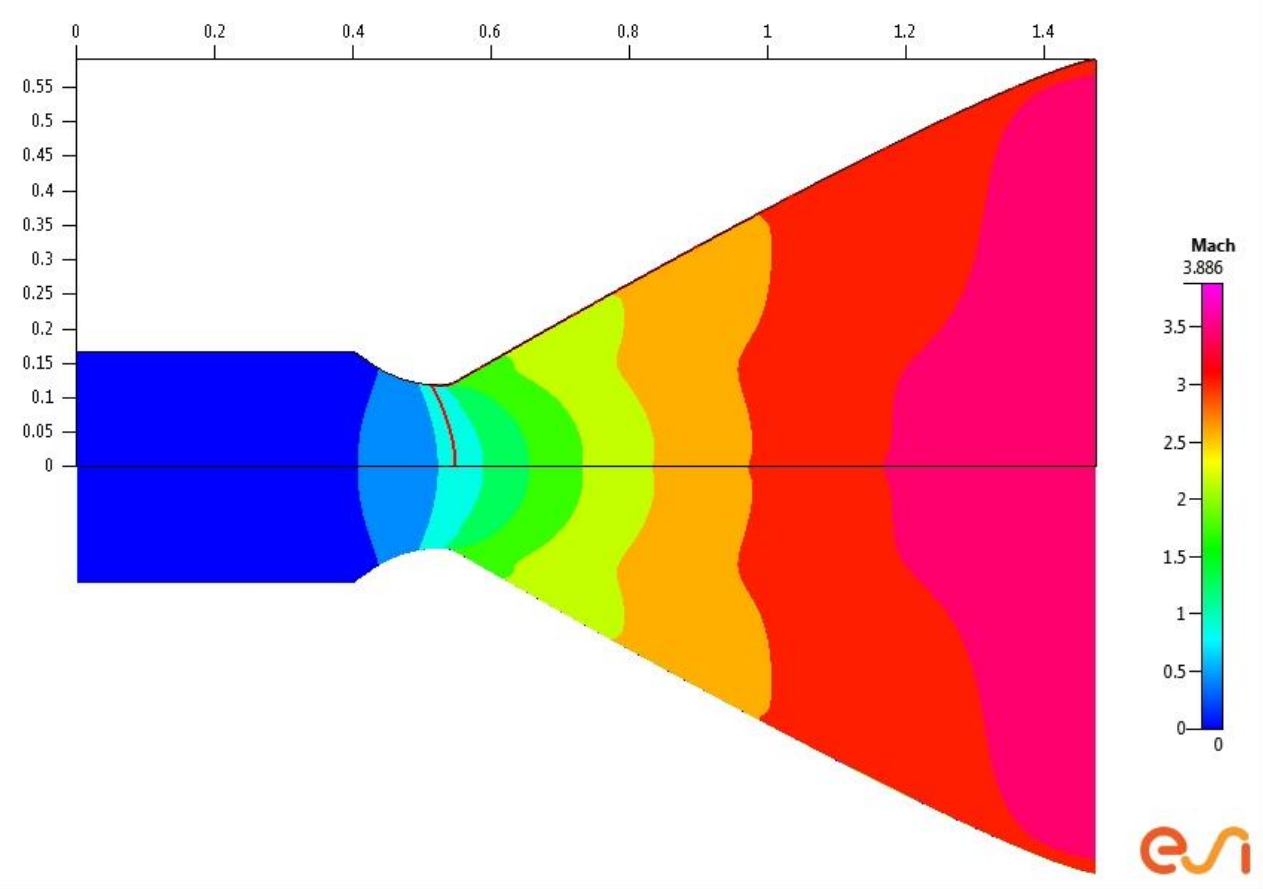

Figure 55 Mach Zones Output for Stage 1 LOX/RP1 
Stage 2 LOX LH2 Output.

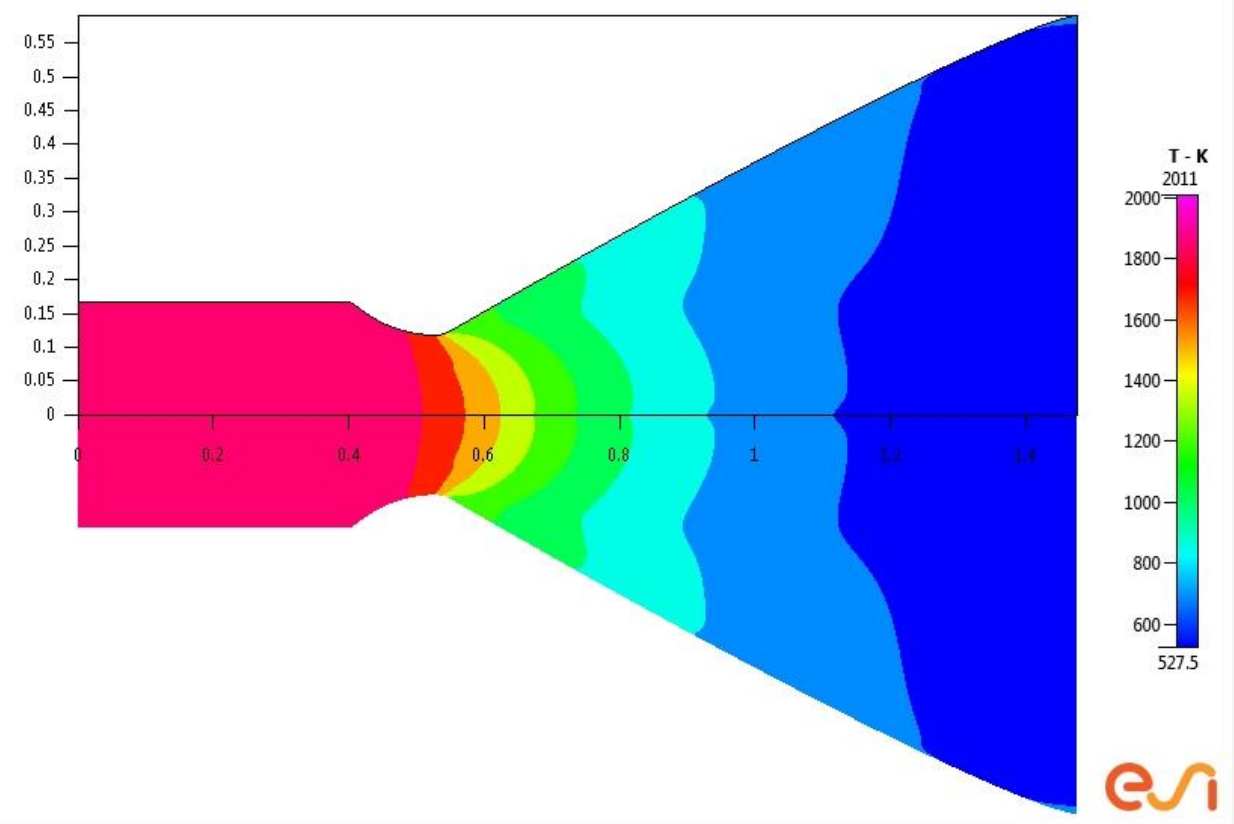

Figure 56 Pressure Map Output for Stage 2 LOX/LH2

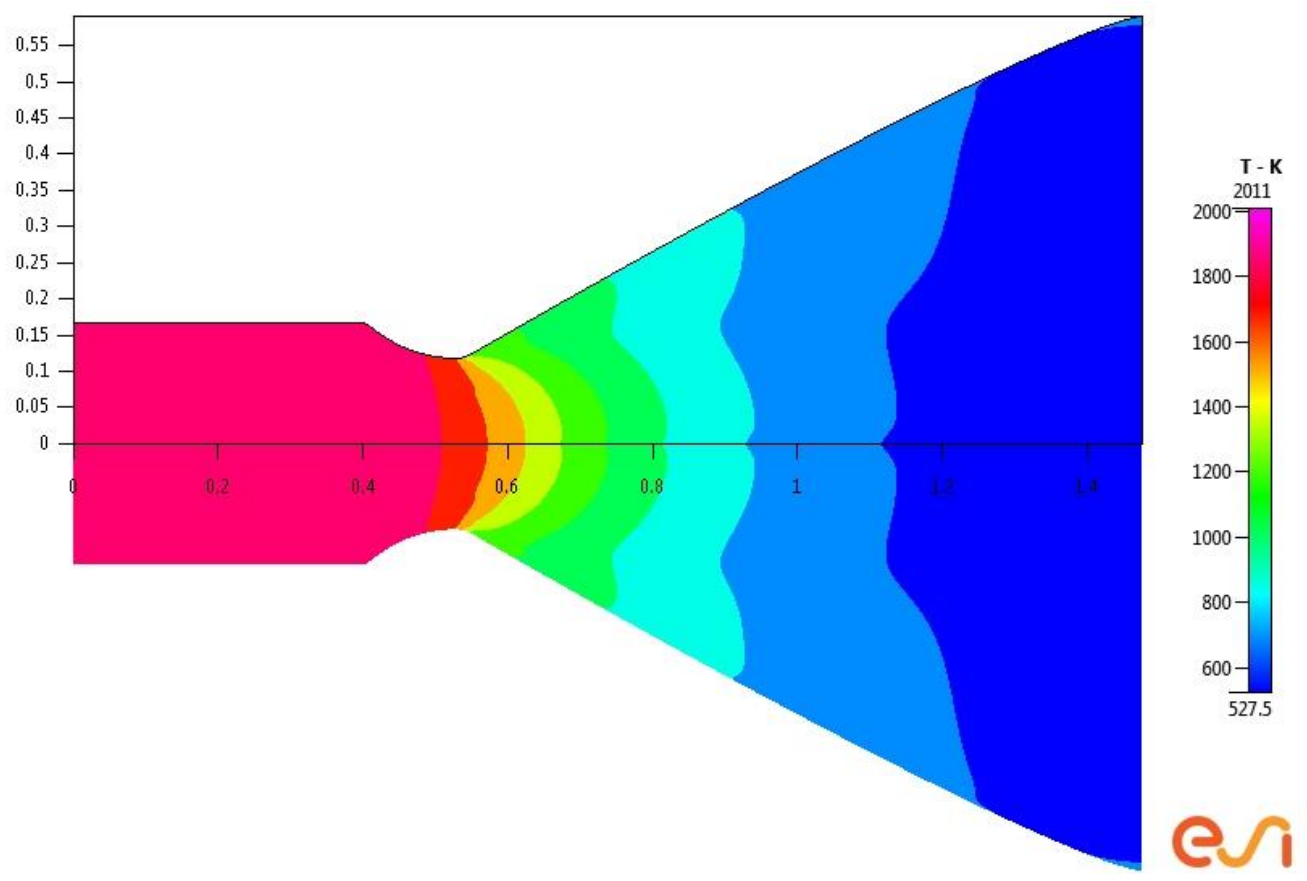

Figure 57 Temperature Map Output for Stage 2 LOX/LH2 


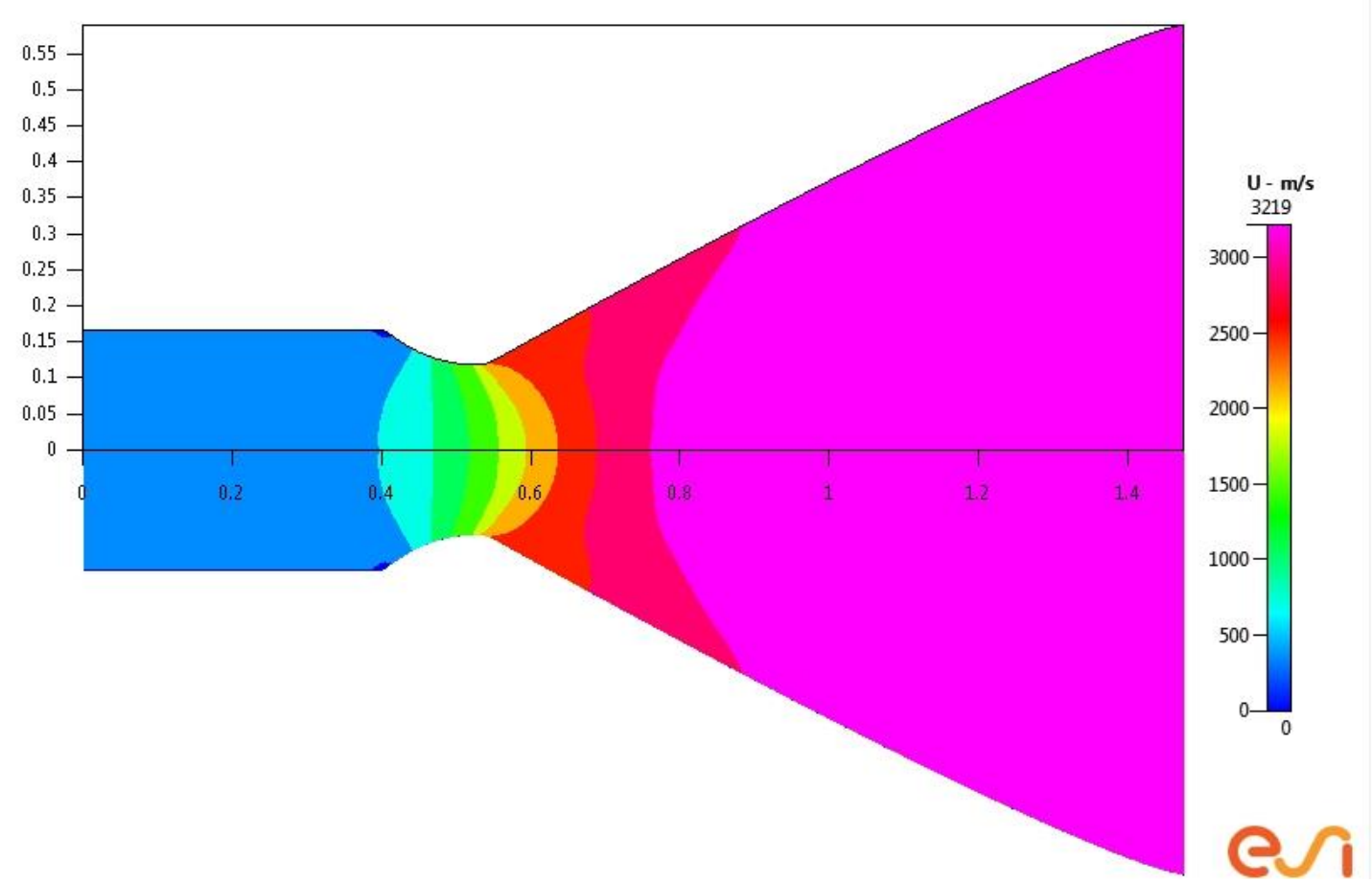

Figure 58 X - Velocity Field Output for Stage 2 LOX/LH2

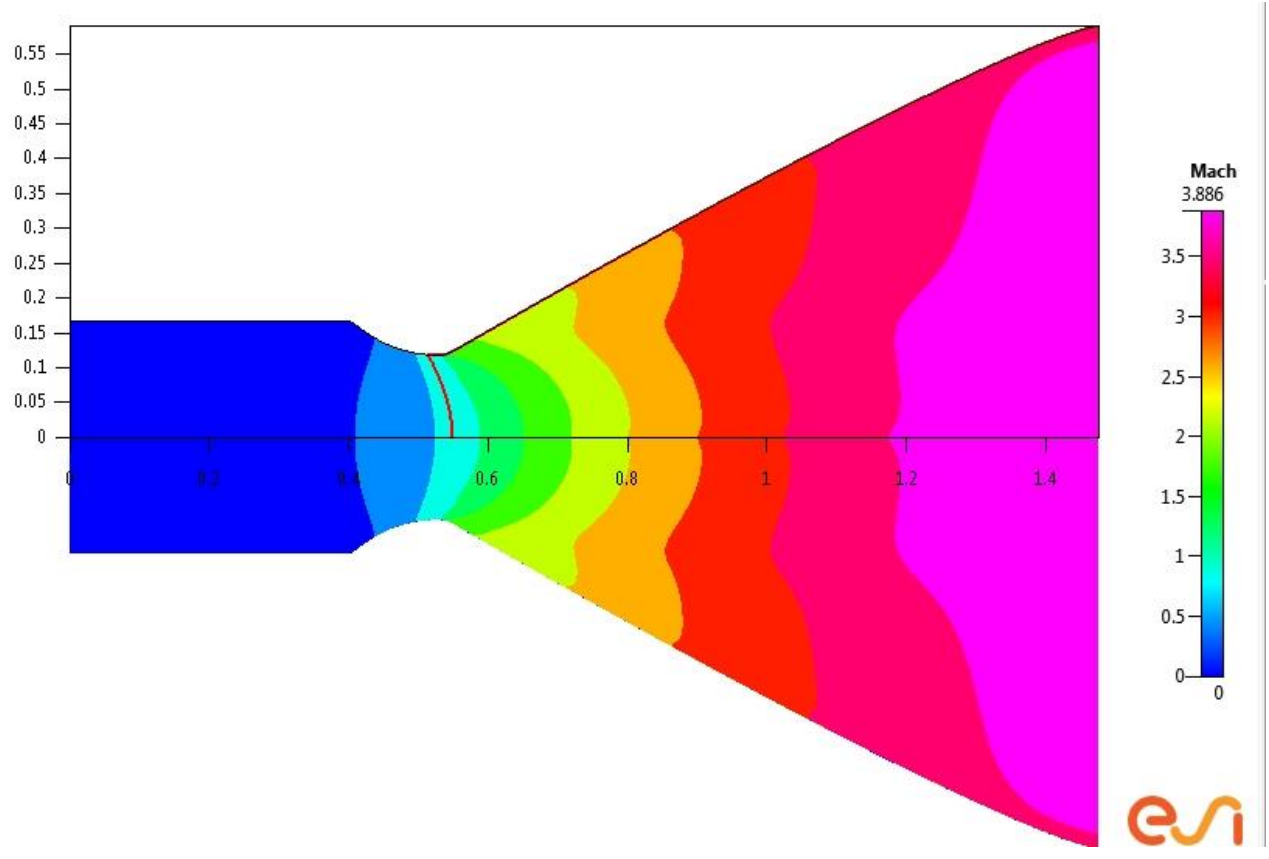

Figure 59 Mach Zones Output for Stage 2 LOX/LH2 
Stage 2 LH2 LF2 Output.

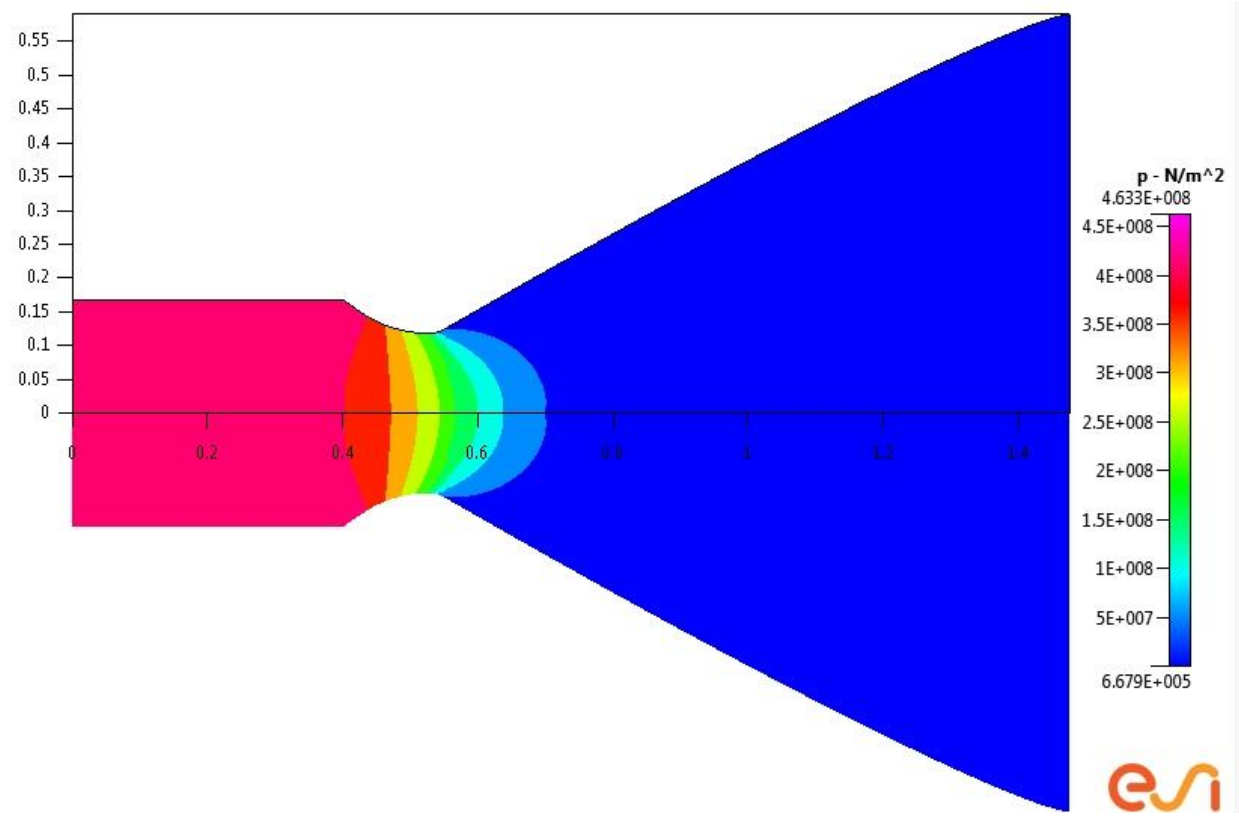

Figure 60 Pressure Map Output for Stage 2 LF2/LH2

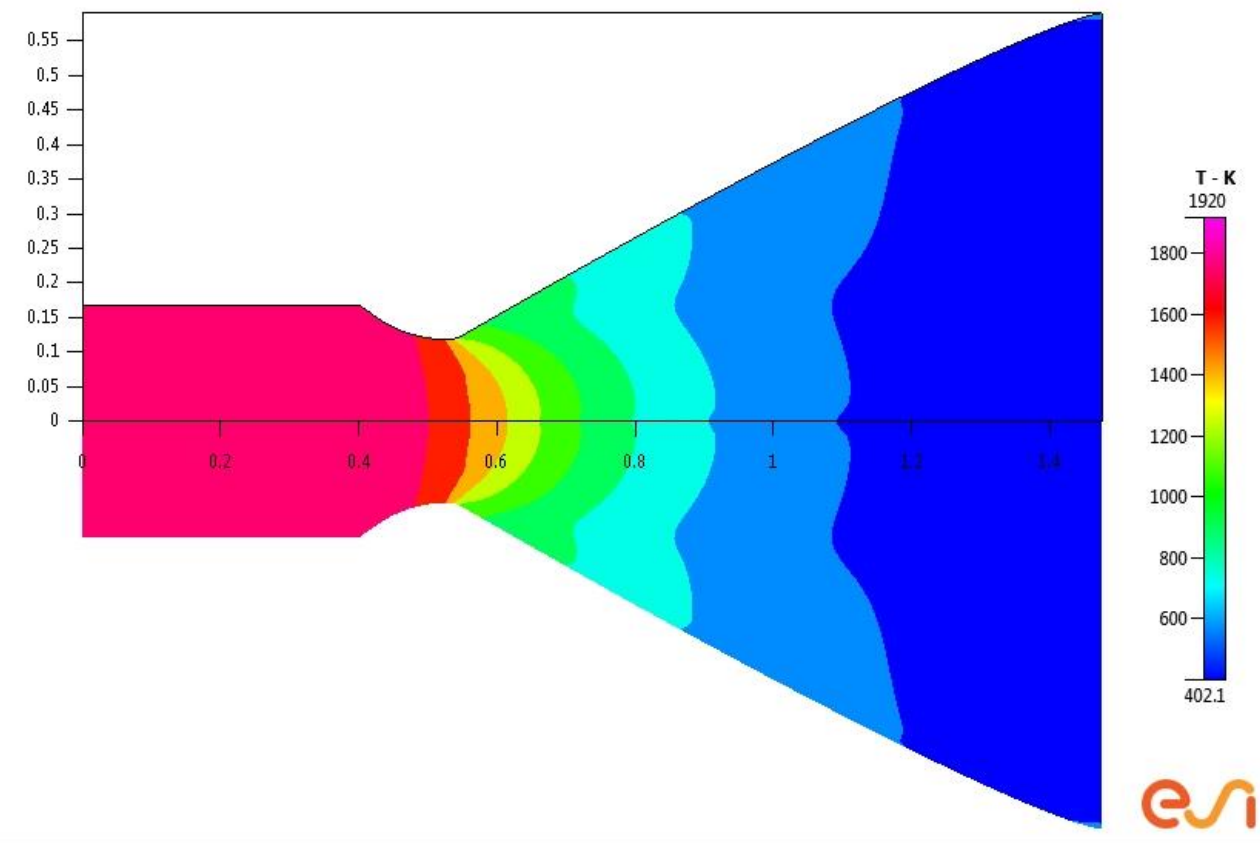

Figure 61 Temperature Map Output for Stage 2 LF2/LH2 


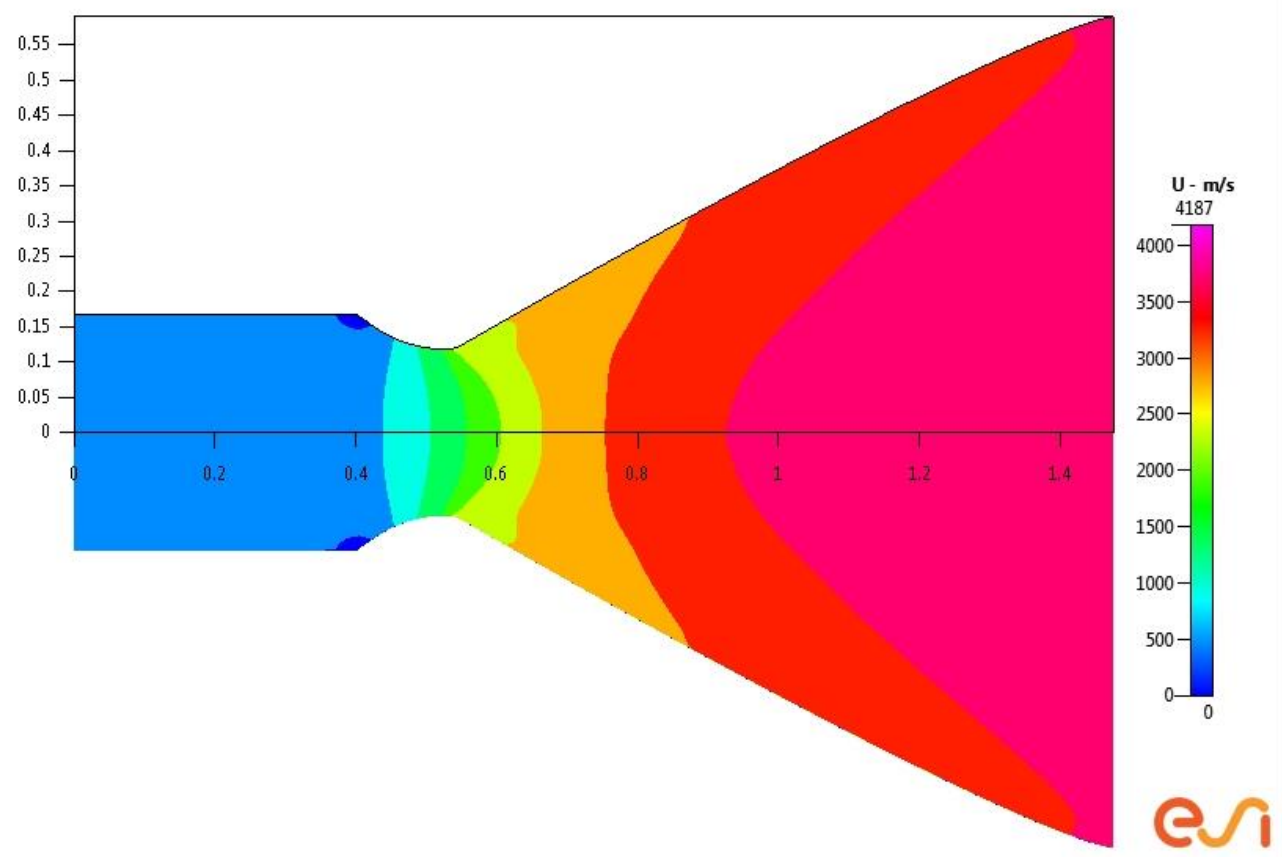

Figure 62 X - Velocity Field for Stage 2 LF2/LH2

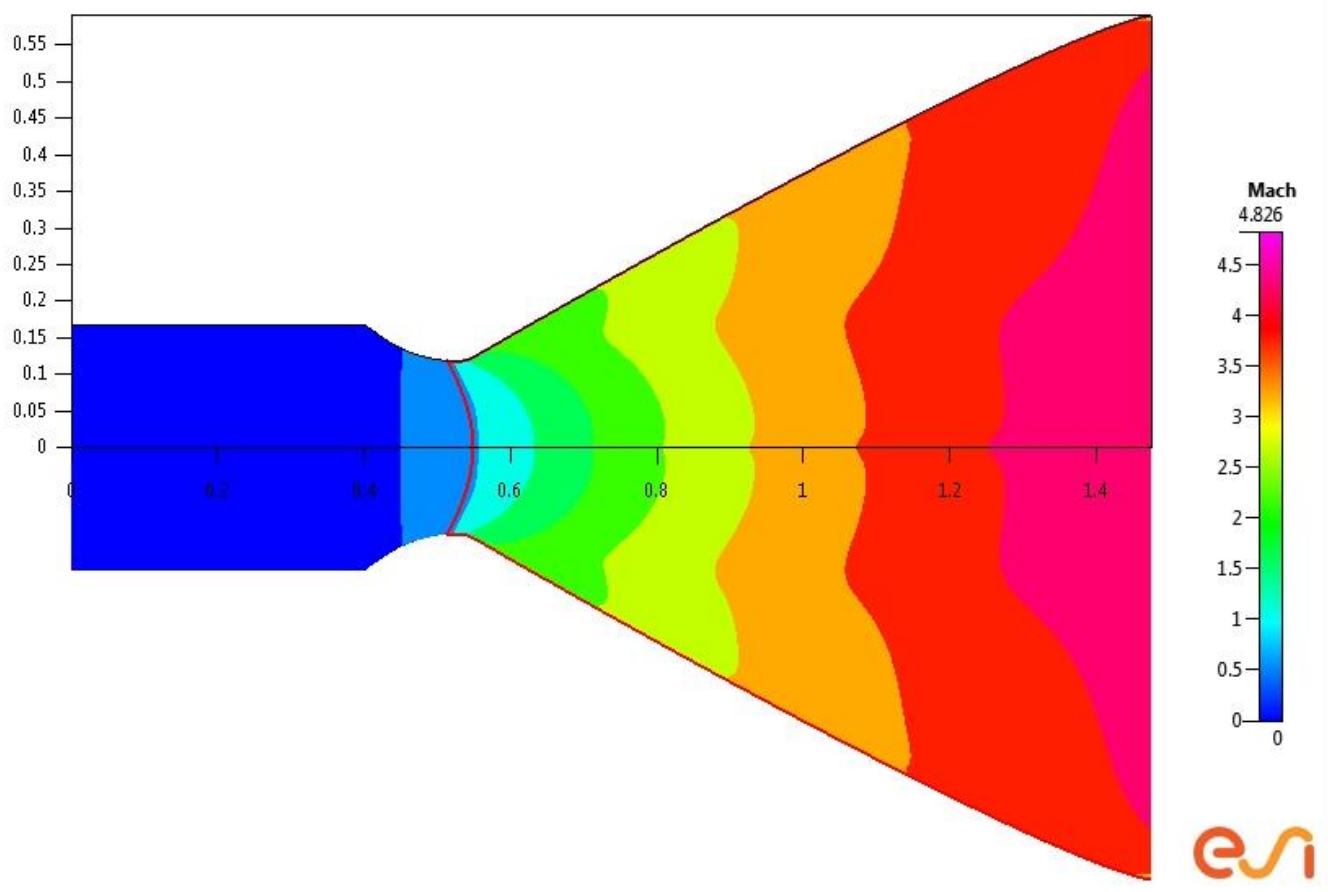

Figure 63 Mach Zones Output for Stage 2 LF2/LH2 


\section{Data from CFD - VIEW Iteration Two}

Observing where the Mach one line is for each of the outputs reveals that an increased pressure is favorable. The Mach line seems to be pushed forward for most cases, but tends to remain in the throat region. There are no glaring Mach one lines moving drastically downstream.

In regards to the other outputs, the pressure, $\mathrm{x}$-velocity fields, and temperature maps all seem to be pushed toward the exit. The higher pressure effects can be best seen in the Mach zones and x-velocity field outputs. There is also a marked increase in the xvelocity field values, which is expected.

Finally, it is important to note that, as in the previous set of outputs, there are small regions in the $\mathrm{x}$-velocity fields that exhibit a sudden drop in velocity, clearly visible in the chambers. This happens when the same contours were used, as in iteration one, so the explanation remains the same as above for the Mach lines. 
Stage 1 LOX RP1 Output.

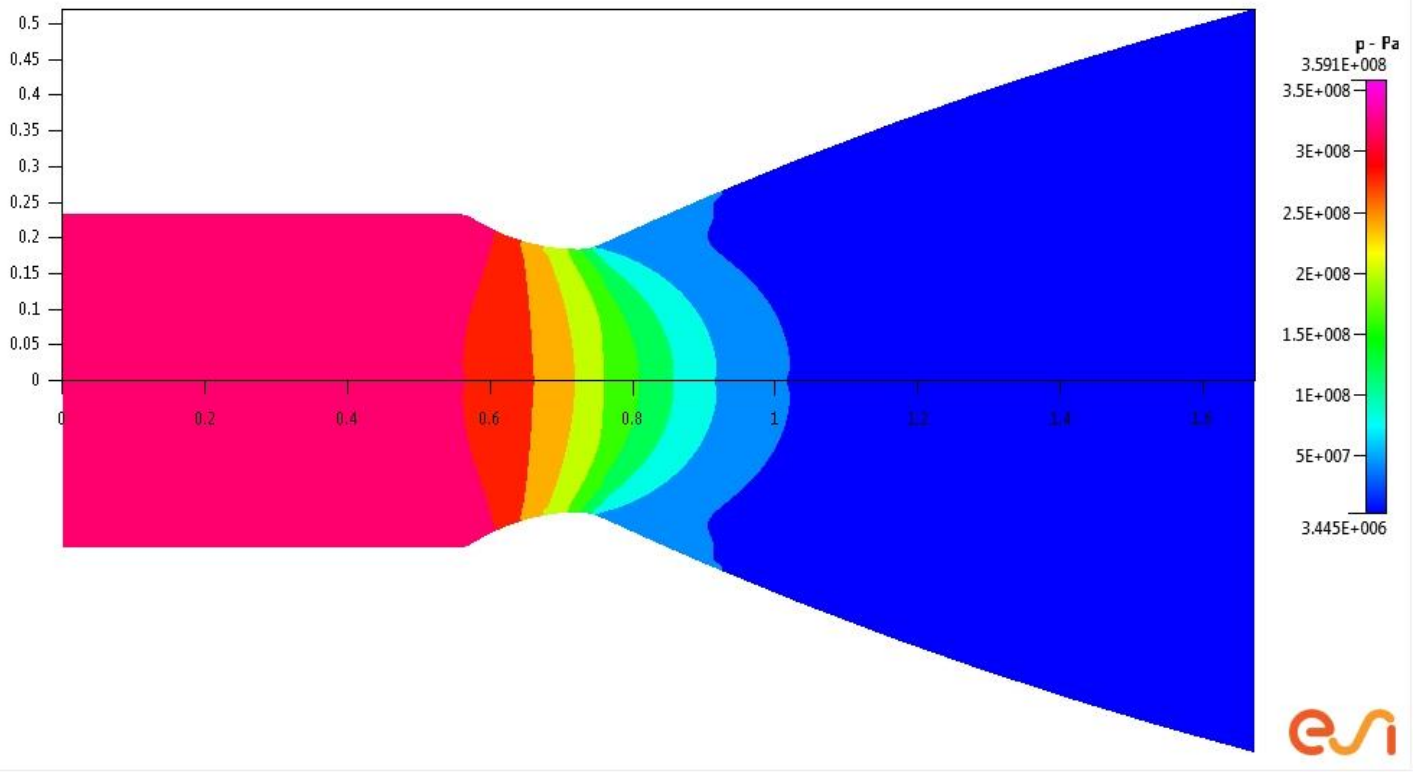

Figure 64 Pressure Map out for Stage 1 LOX/RP1

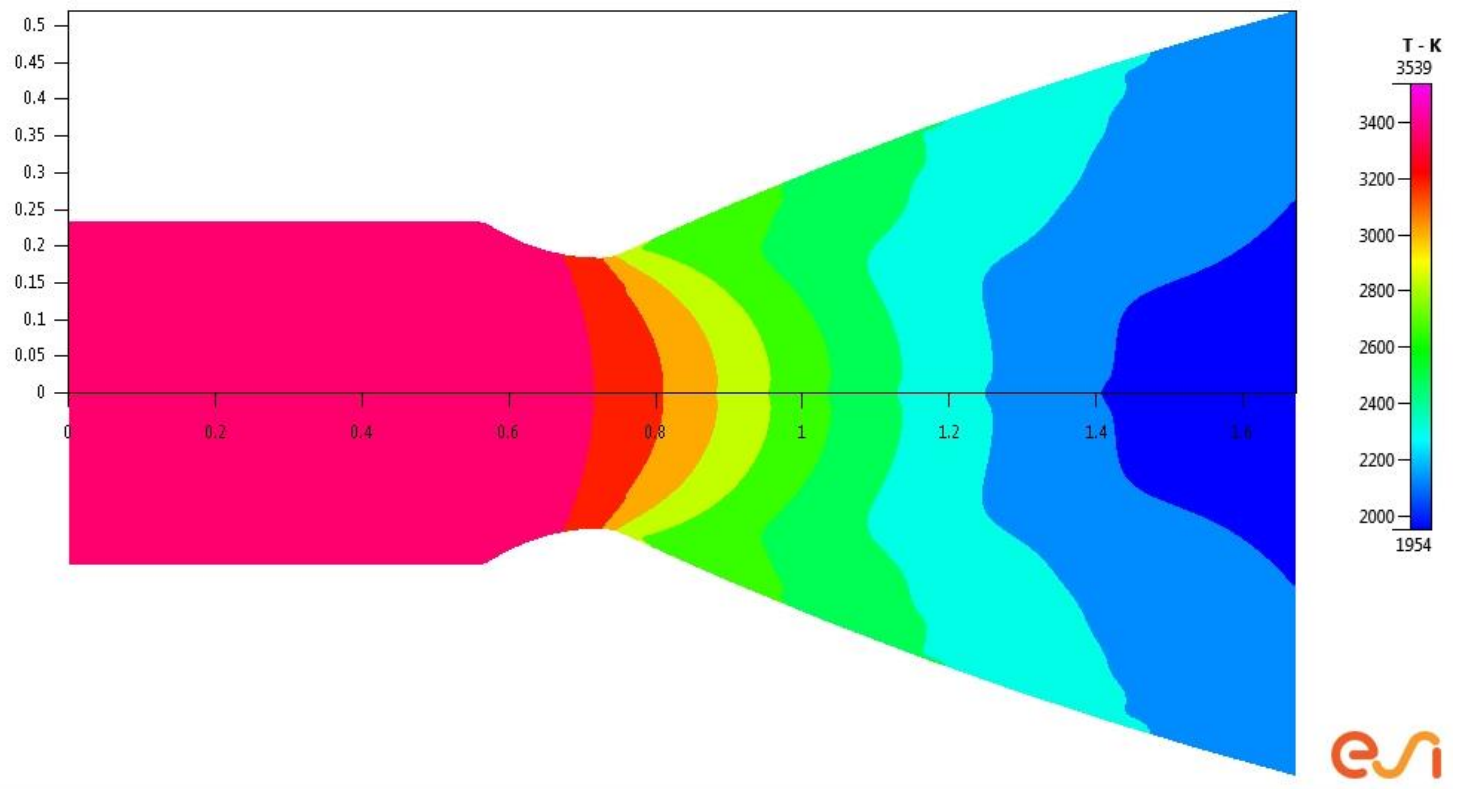

Figure 65 Temperature Map Output for Stage 1 LOX/RP1 


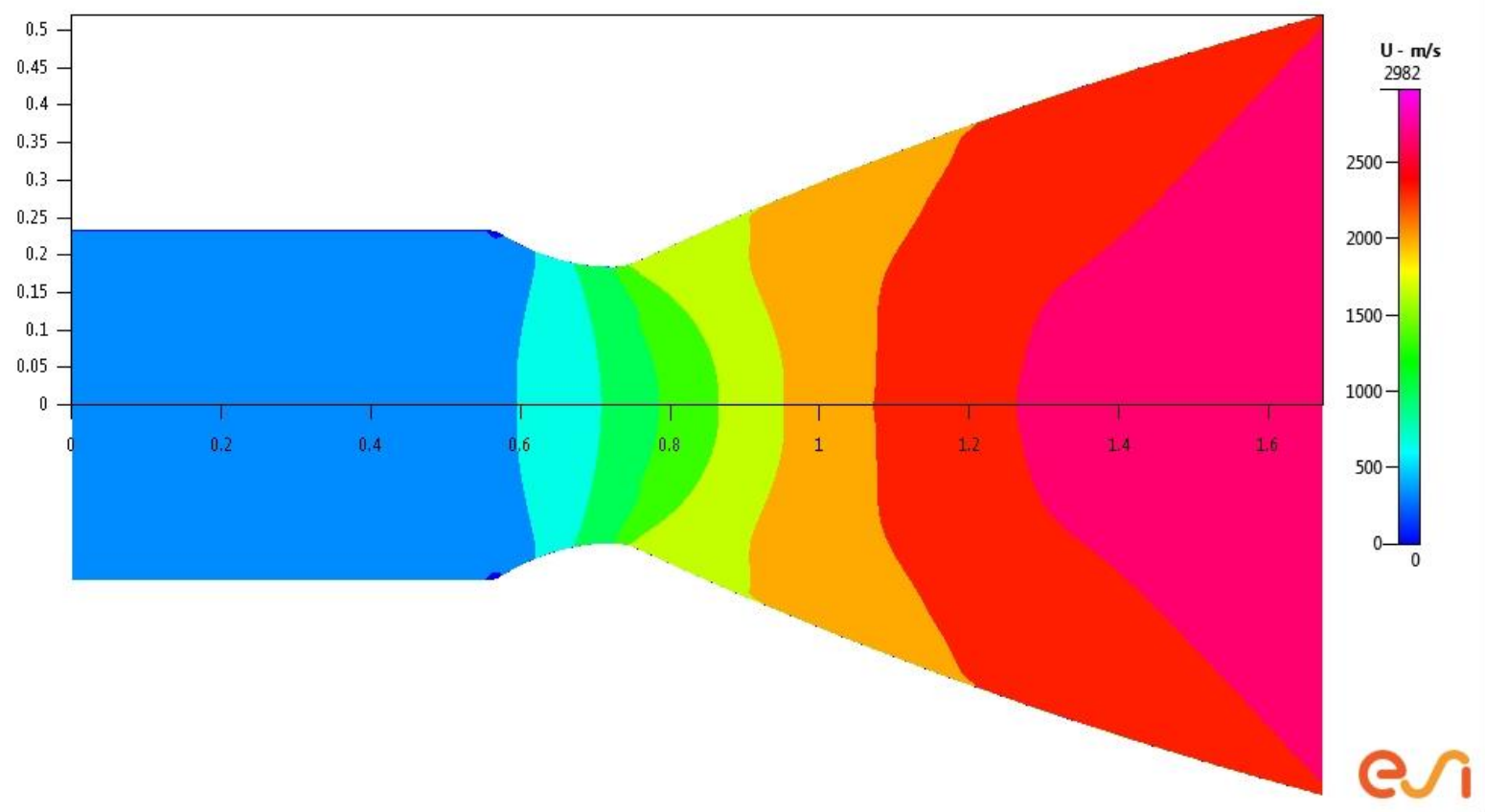

Figure 66 X - Velocity Field for Stage 1 LOX/RP1

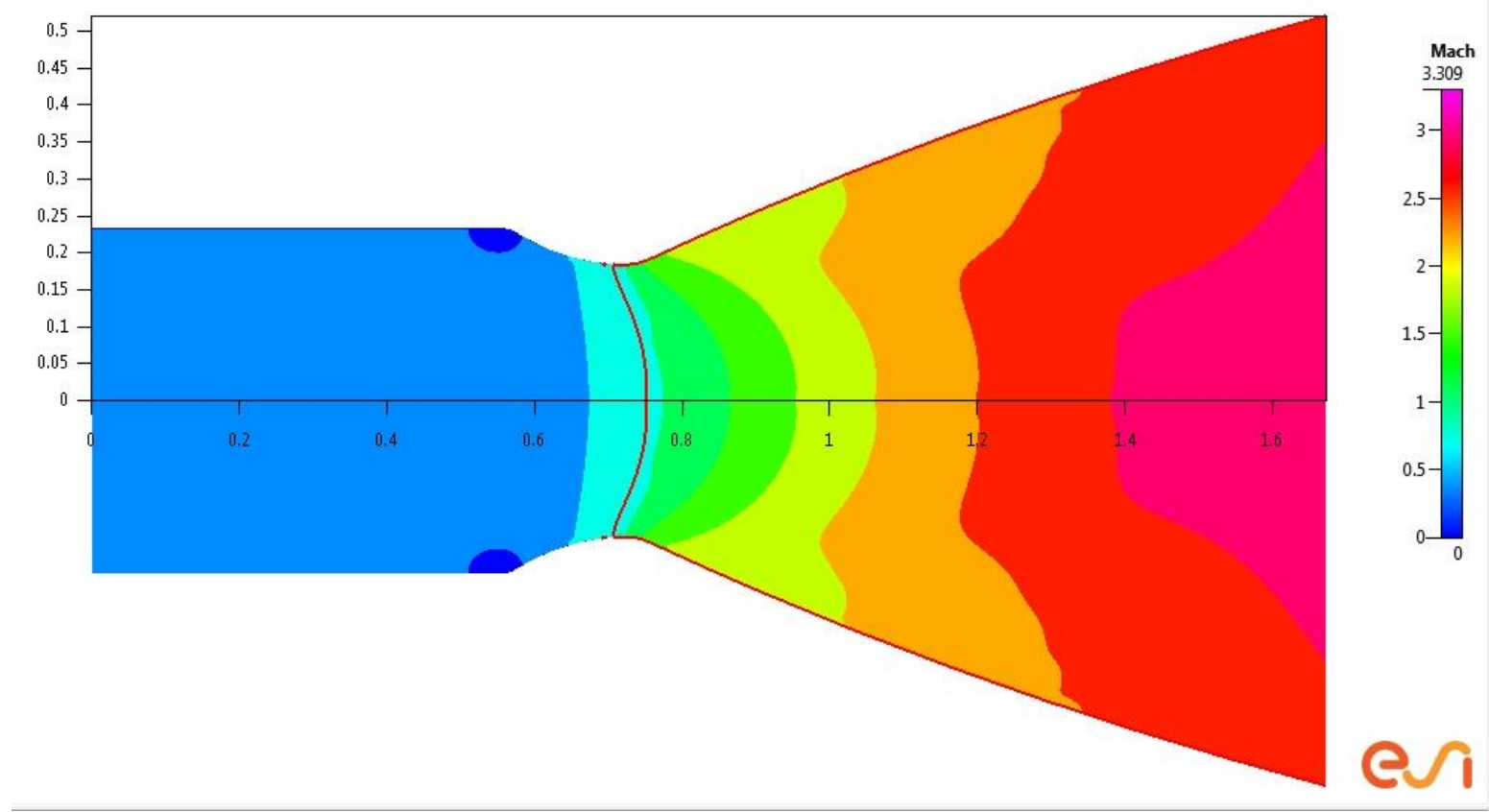

Figure 67 Mach Zones Output for Stage 1 LOX/RP1 
Stage 1 LOX LH2 Output.

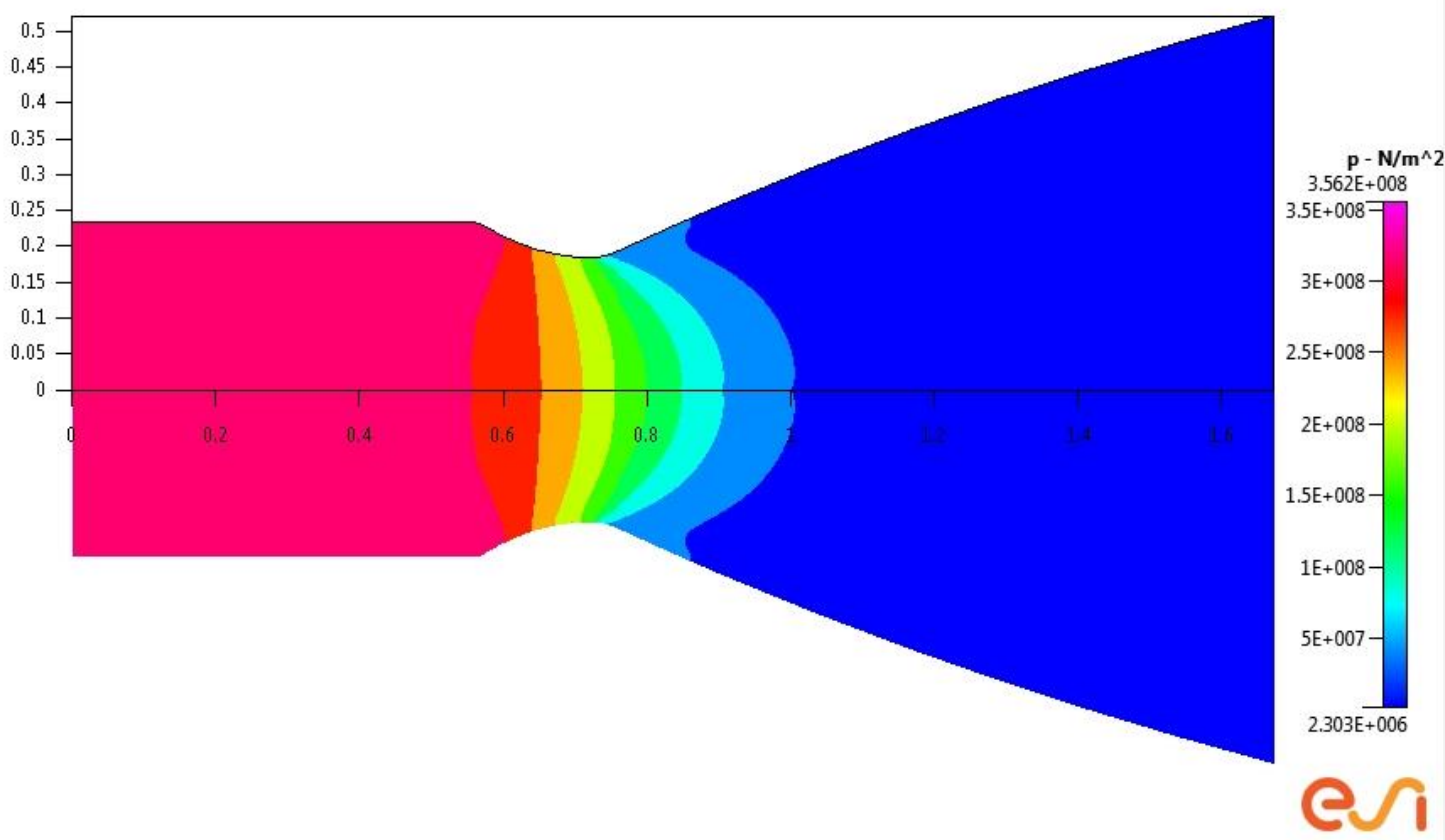

Figure 68 Pressure Map Output for Stage 1 LOX/LH2

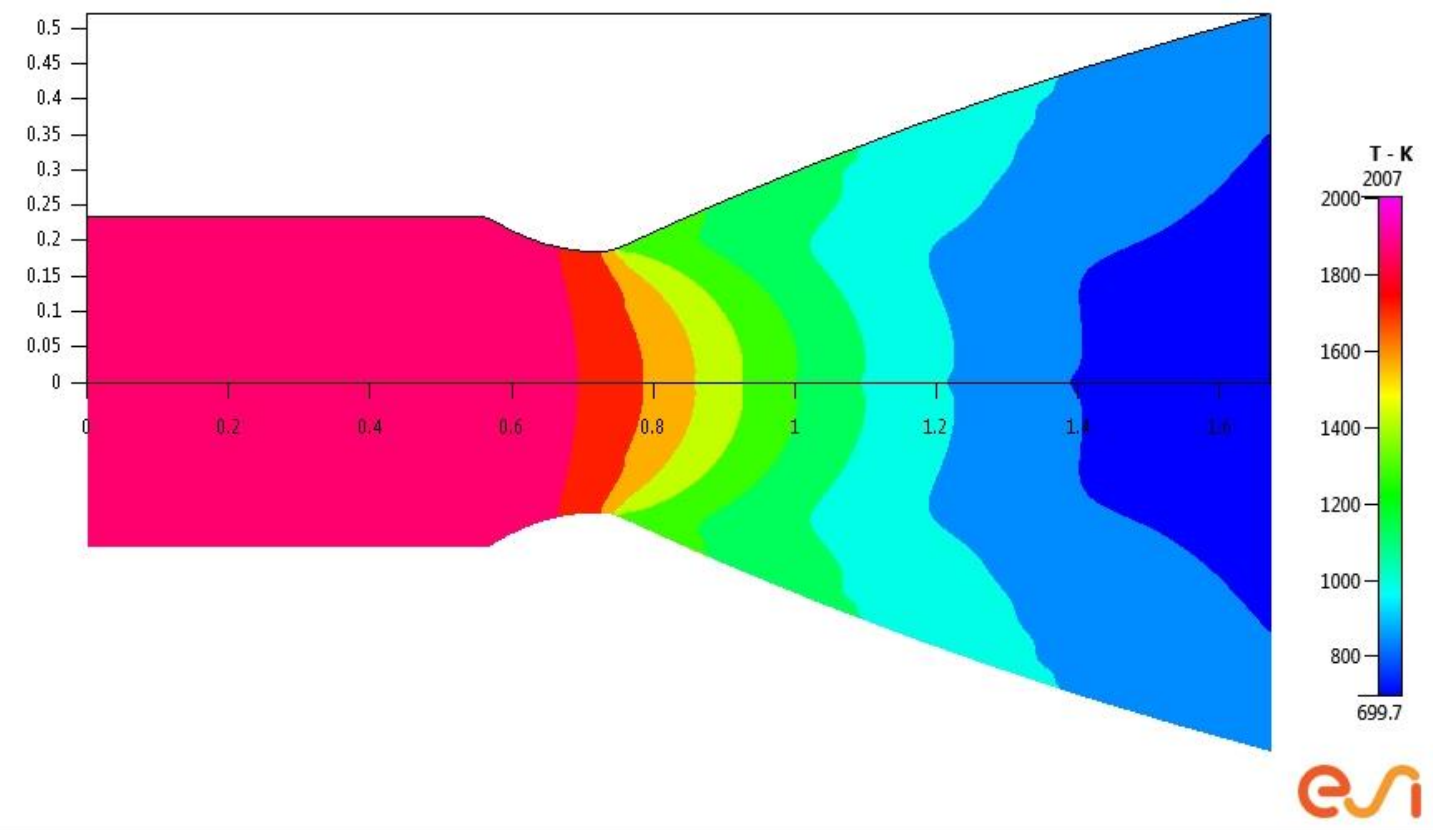

Figure 69 Temperature Map Output for Stage 1 LOX/LH2 


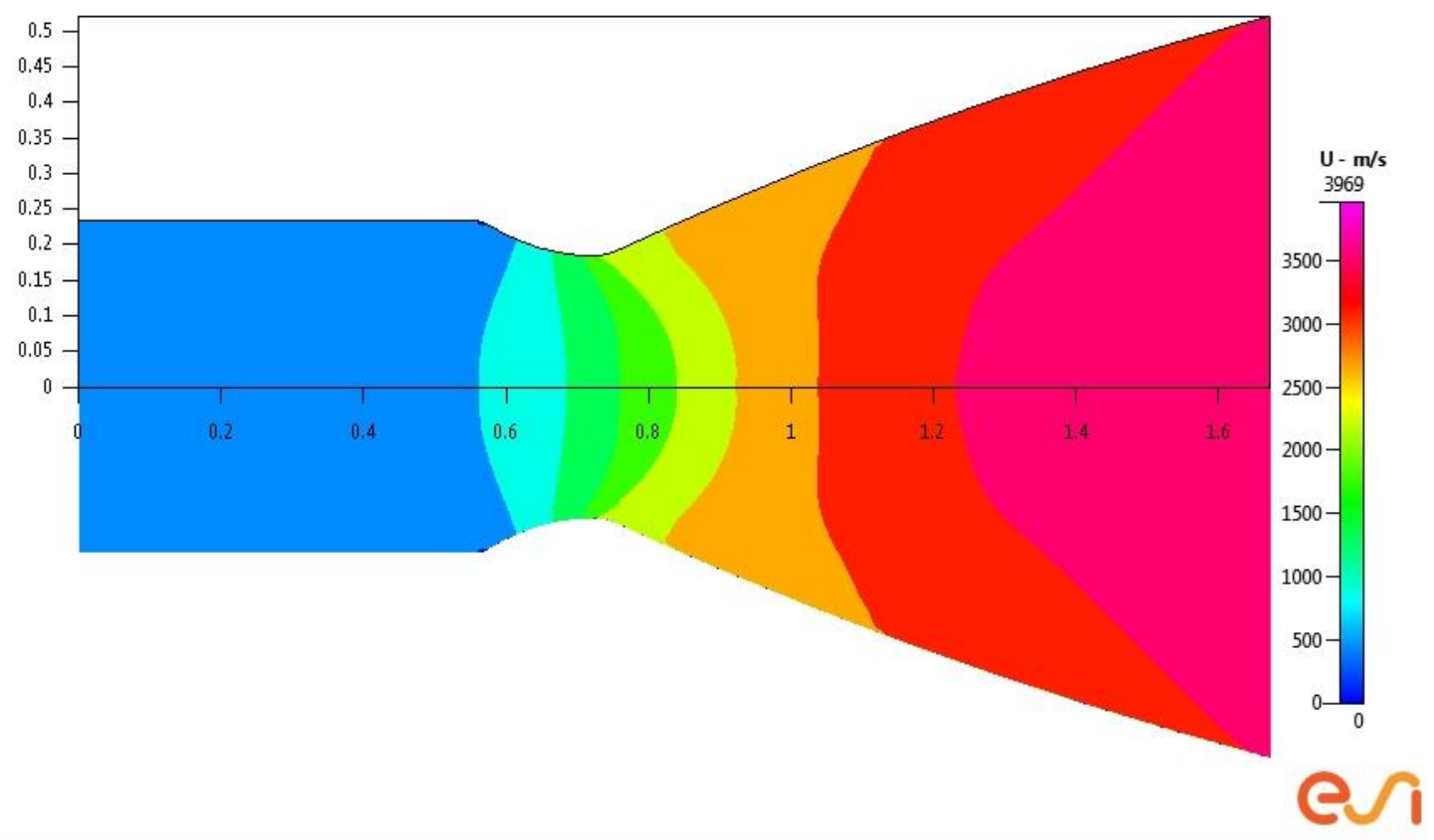

Figure $70 \mathrm{X}$ - Velocity Field for Stage 1 LOX/LH2

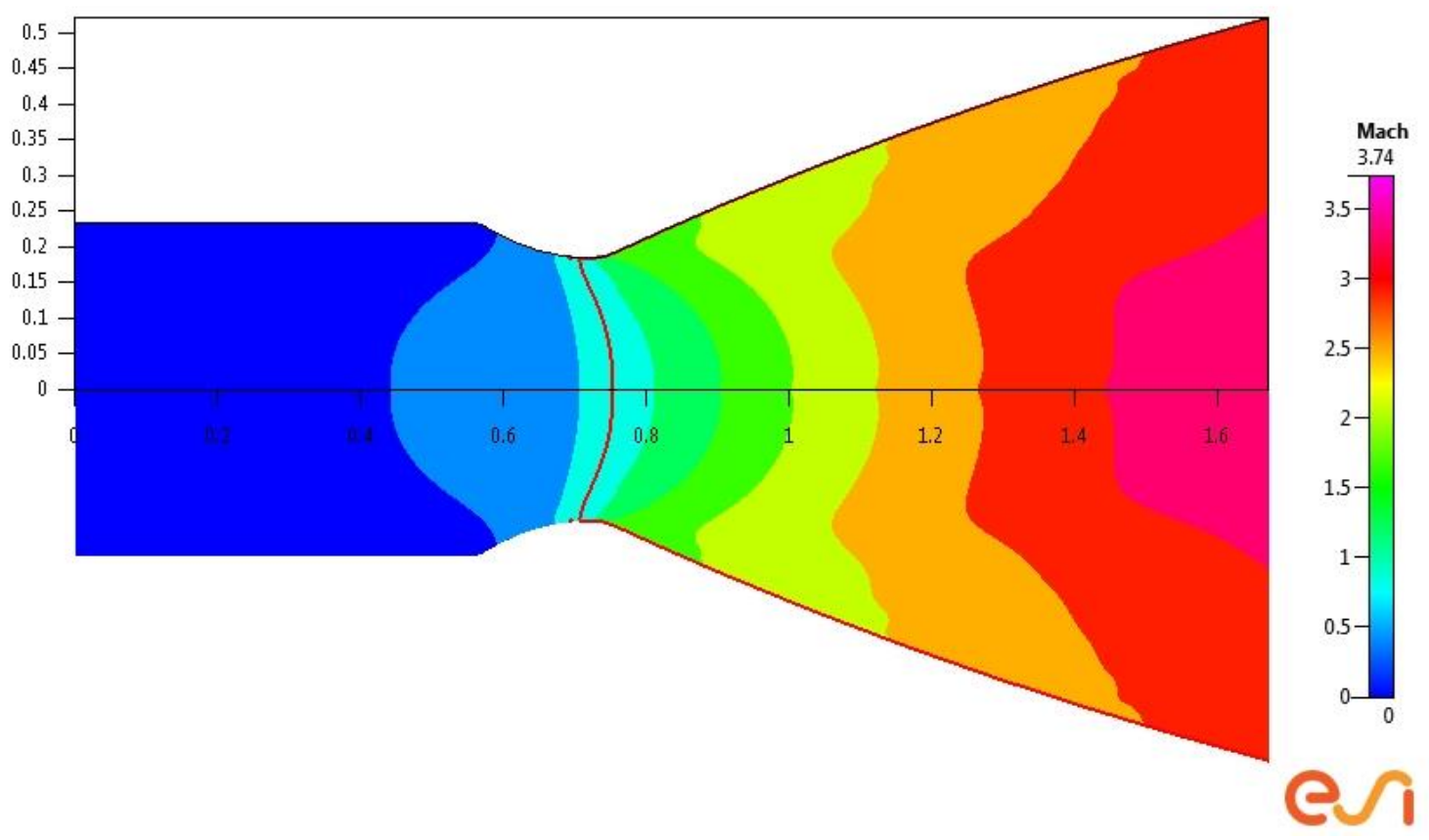

Figure 71 Mach Zones Output for Stage 1 LOX/LH2 
Stage 1 LH2 LF2 Output.

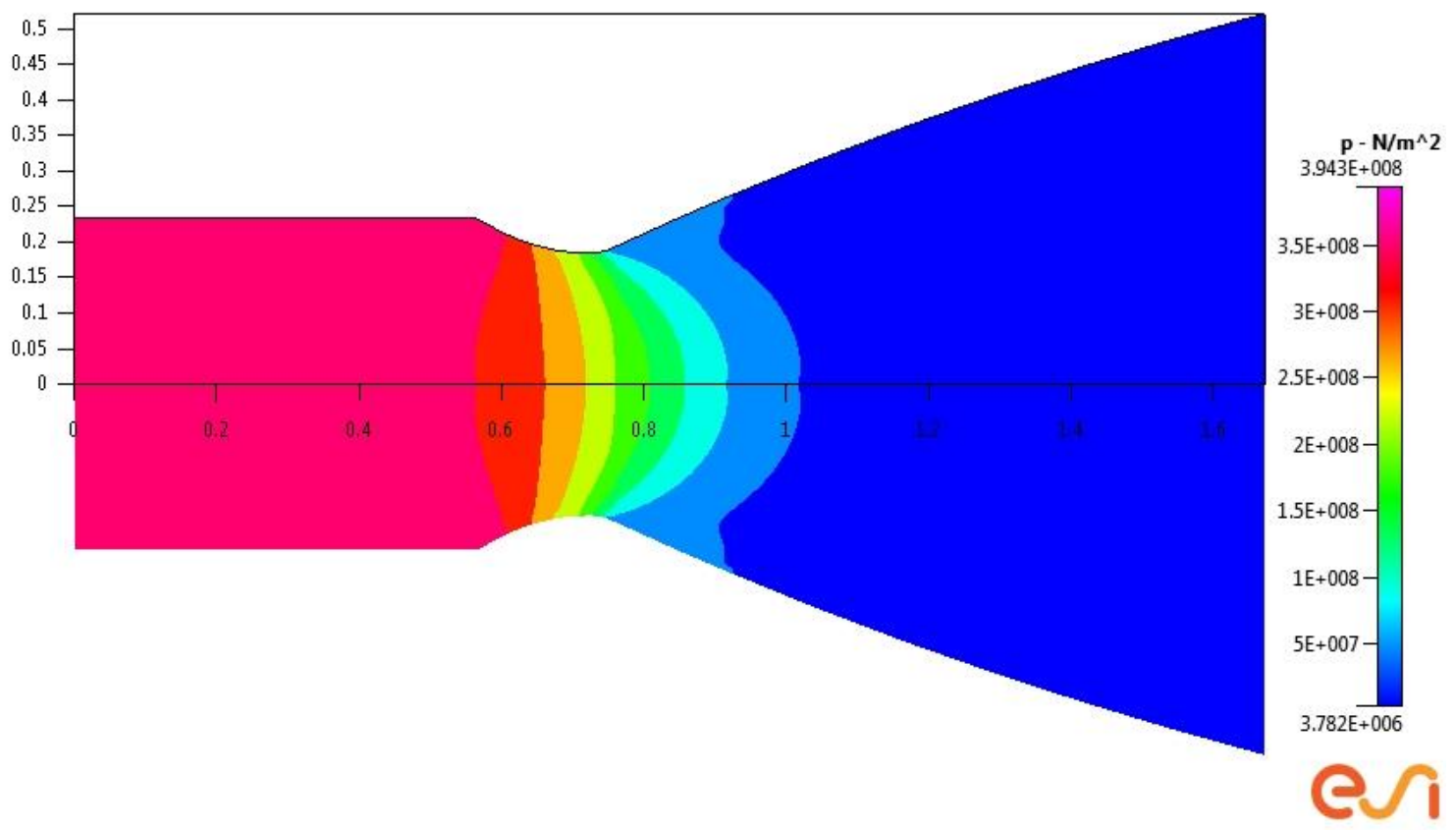

Figure 72 Pressure Map Output for Stage 1 LH2/LF2

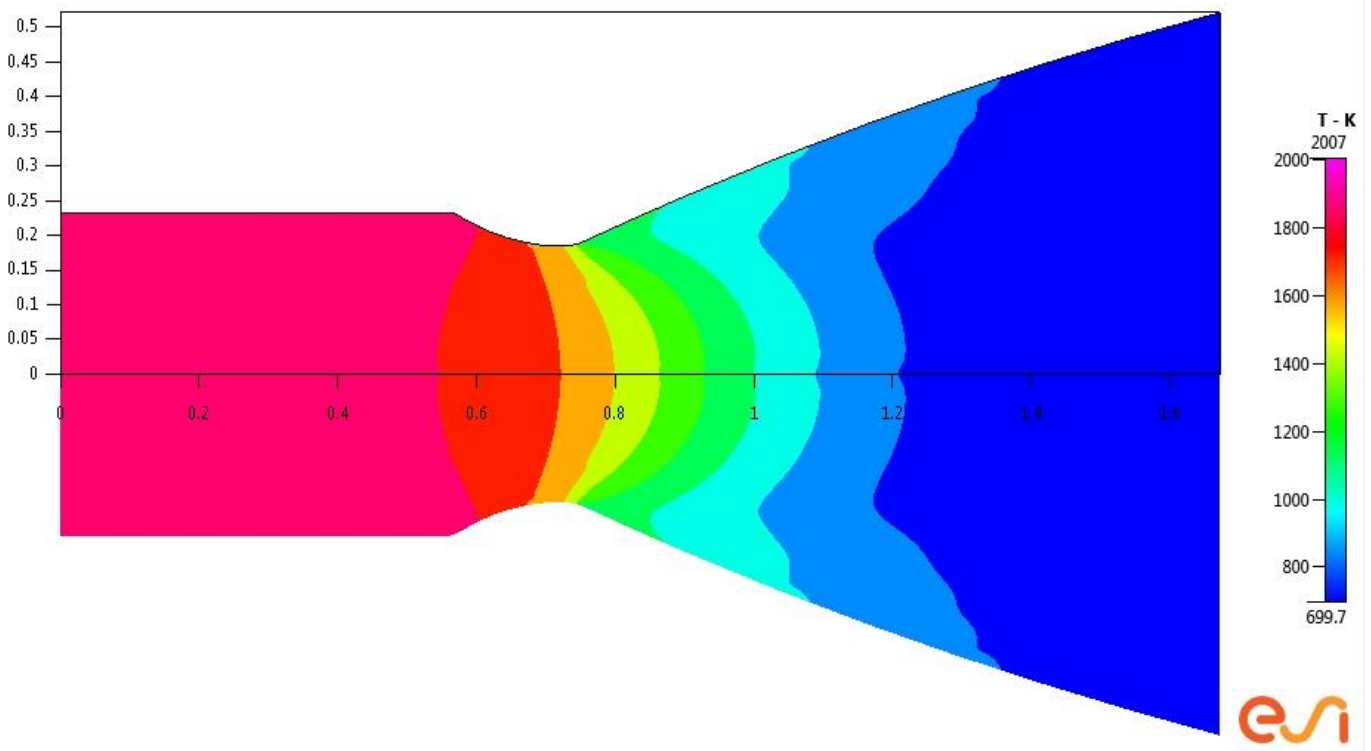

Figure 73 Temperature Map Output for Stage 1 LH2/LF2 

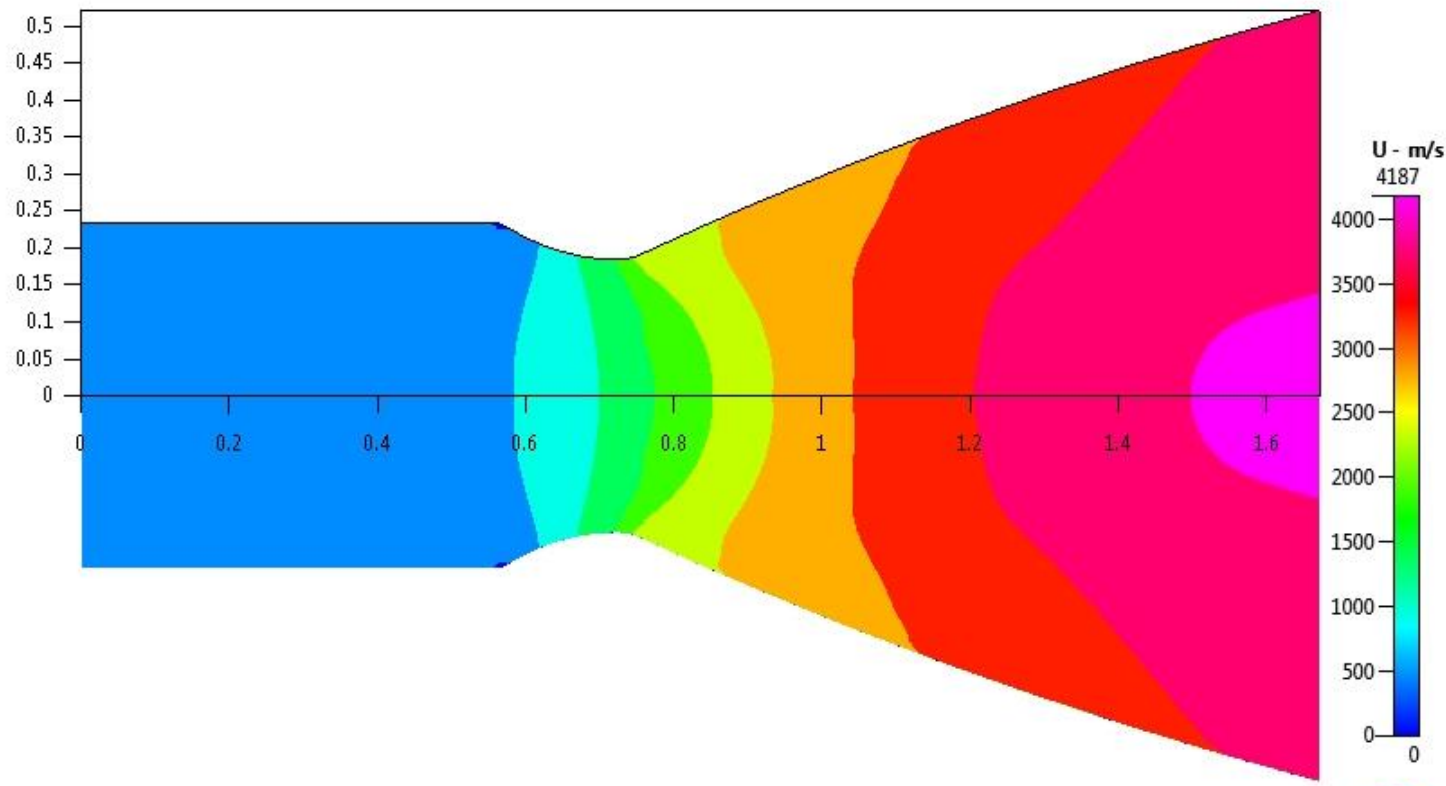

Figure 74 X - Velocity Field for Stage 1 LH2/LF2
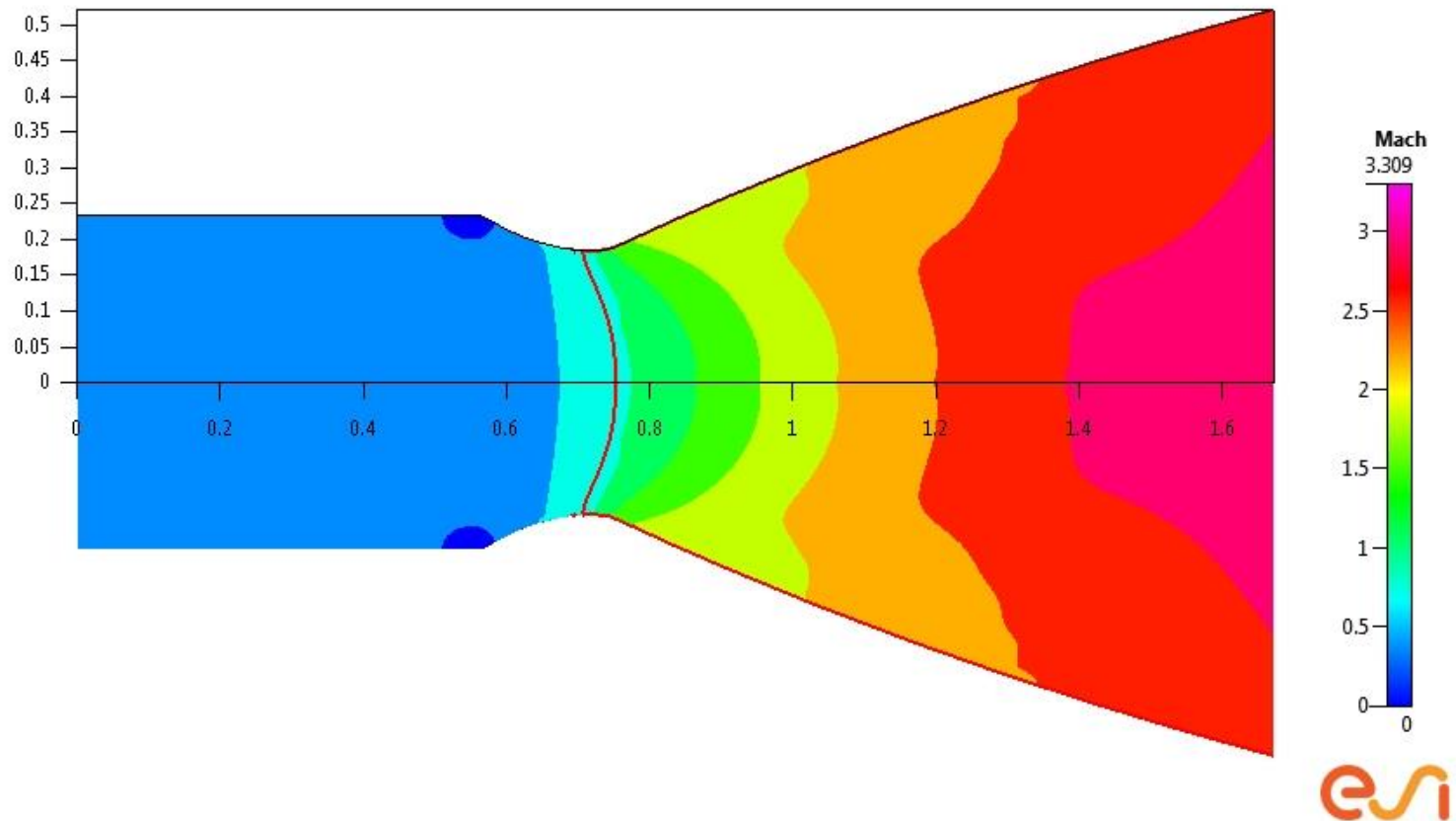

Figure 75 Mach Zones Output for Stage 1 LH2/LF2 
Stage 2 LOX RP1 Output.

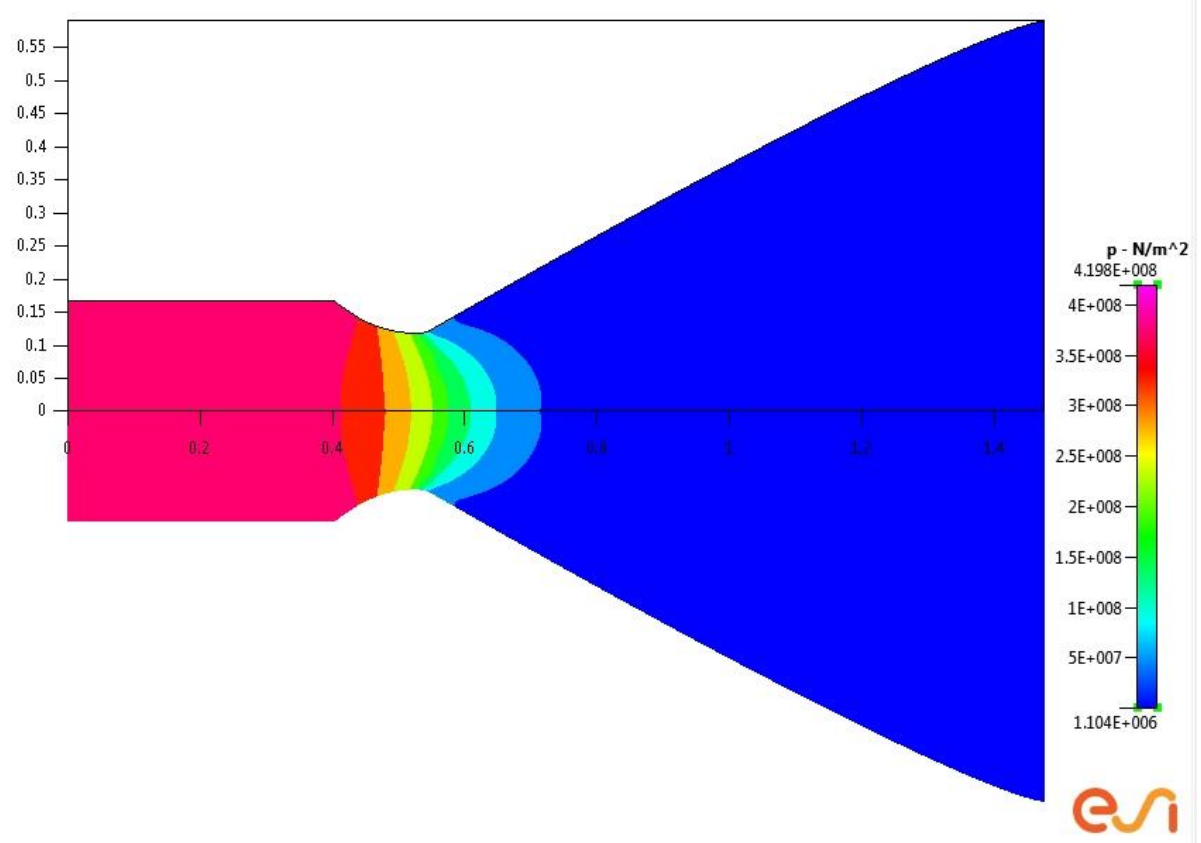

Figure 76 Pressure Map Output for Stage 2 LOX/RP1

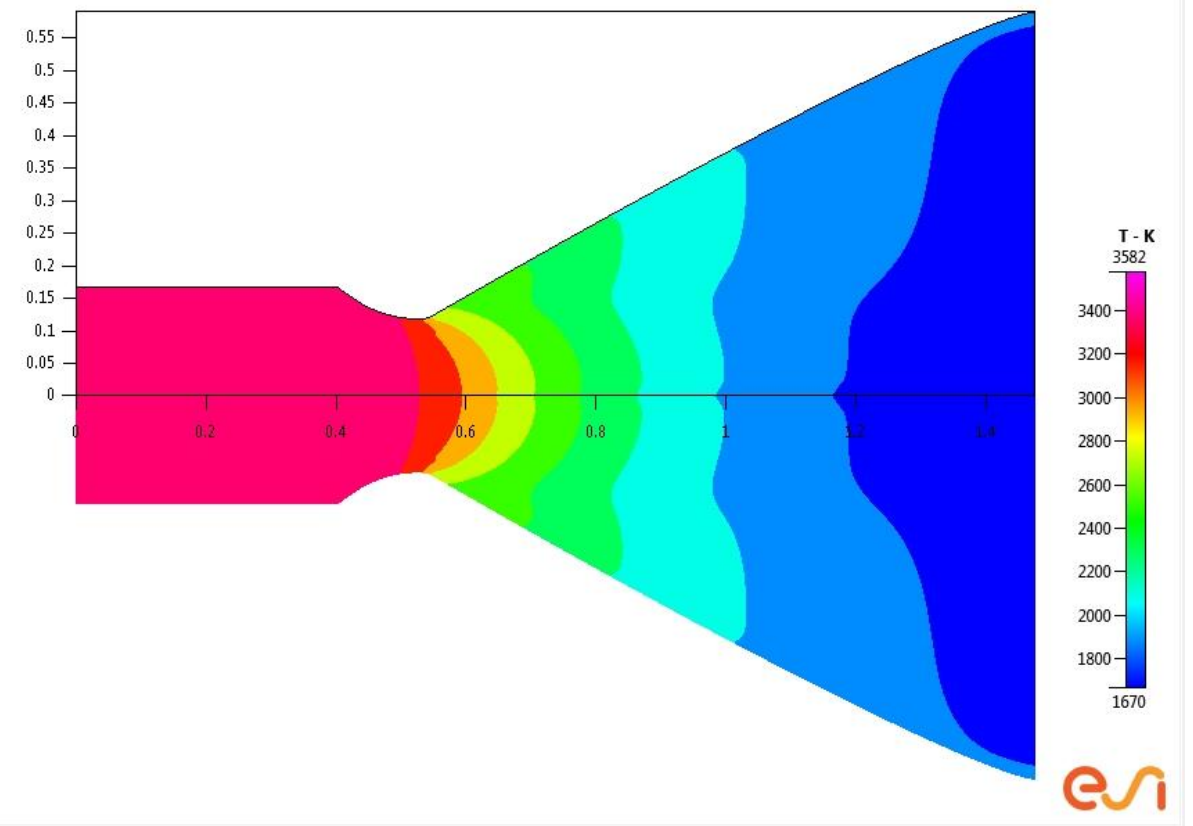

Figure 77 Temperature Map Output for Stage 2 LOX/RP1 


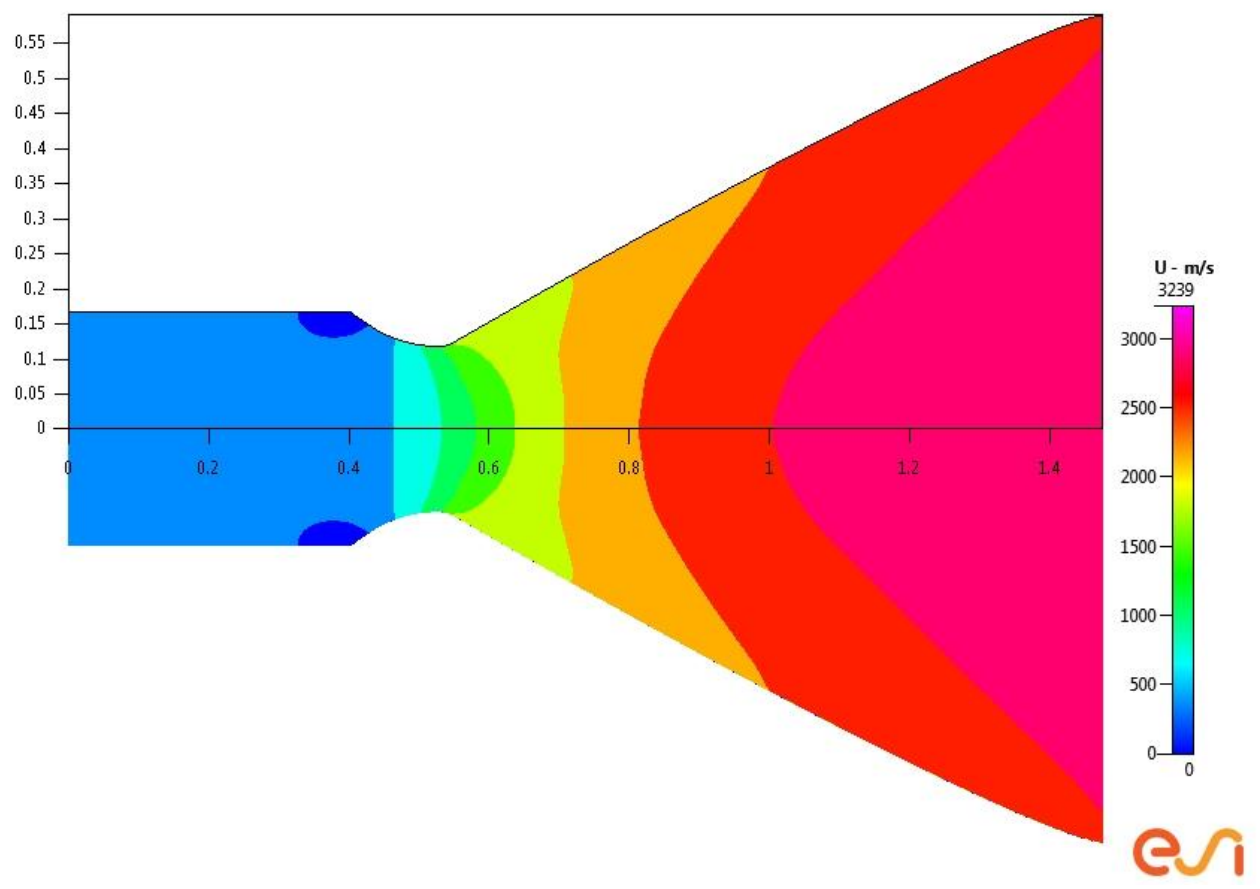

Figure 78 X - Velocity Field Output for Stage 2 LOX/RP1

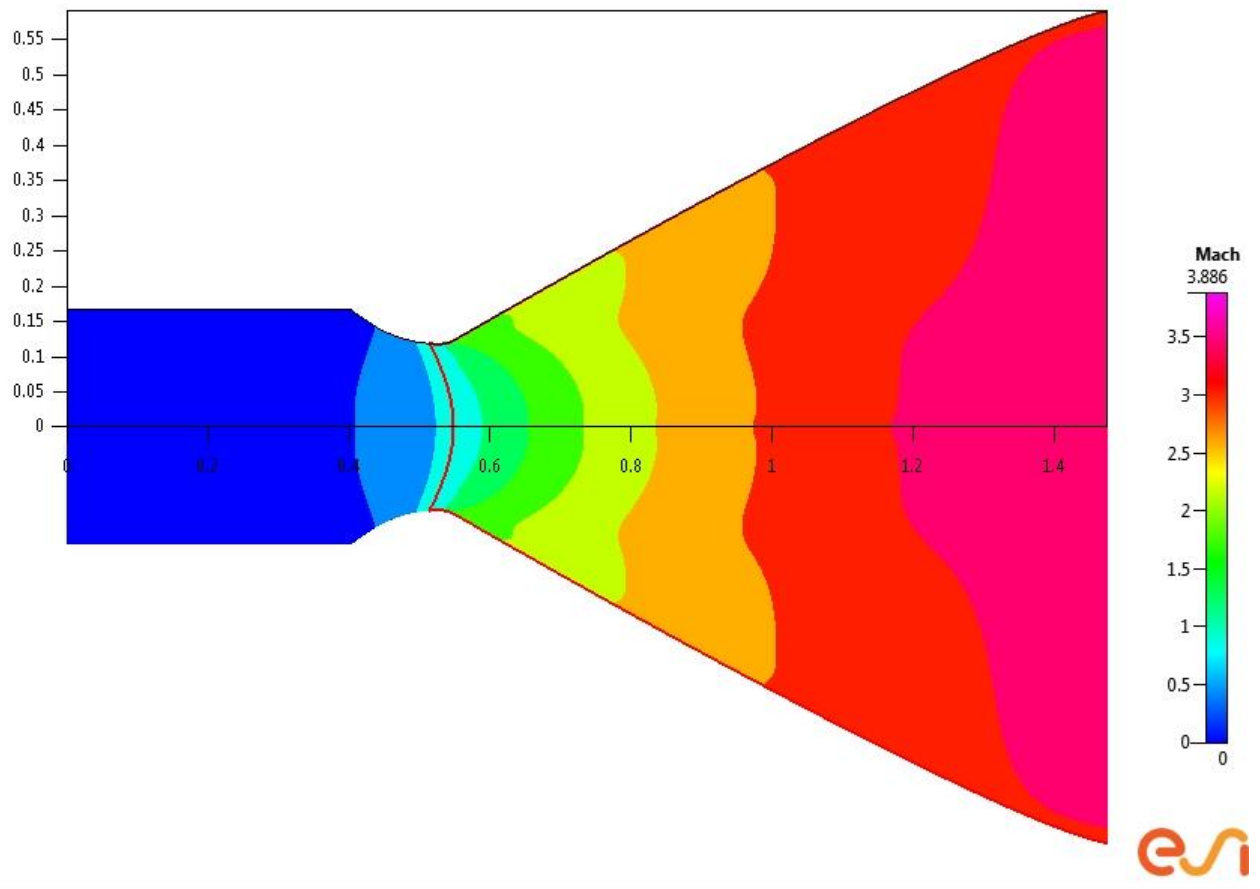

Figure 79 Mach Zones Output for Stage 2 LOX/RP1 
Stage 2 LOX LH2 Output.

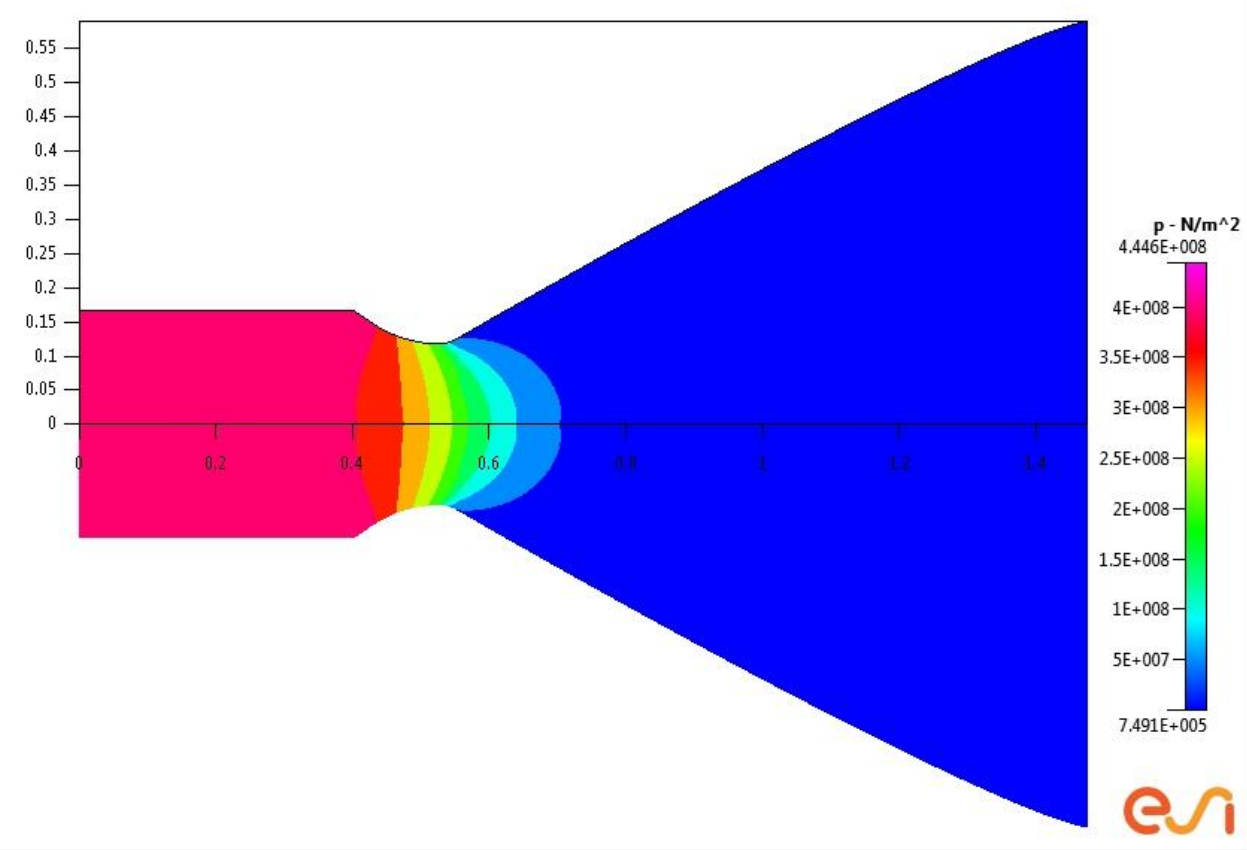

Figure 80 Pressure Map Output for Stage 2 LOX/LH2

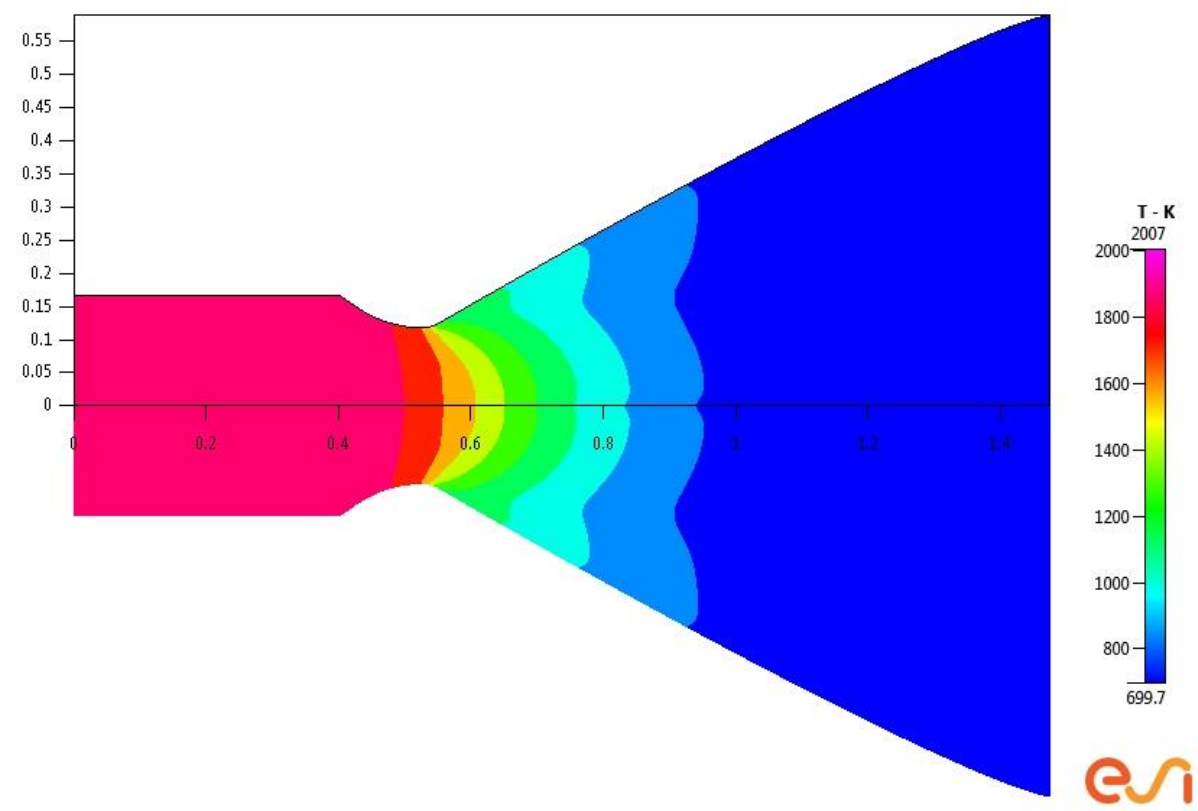

Figure 81 Temperature Map Output for Stage 2 LOX/LH2 106 


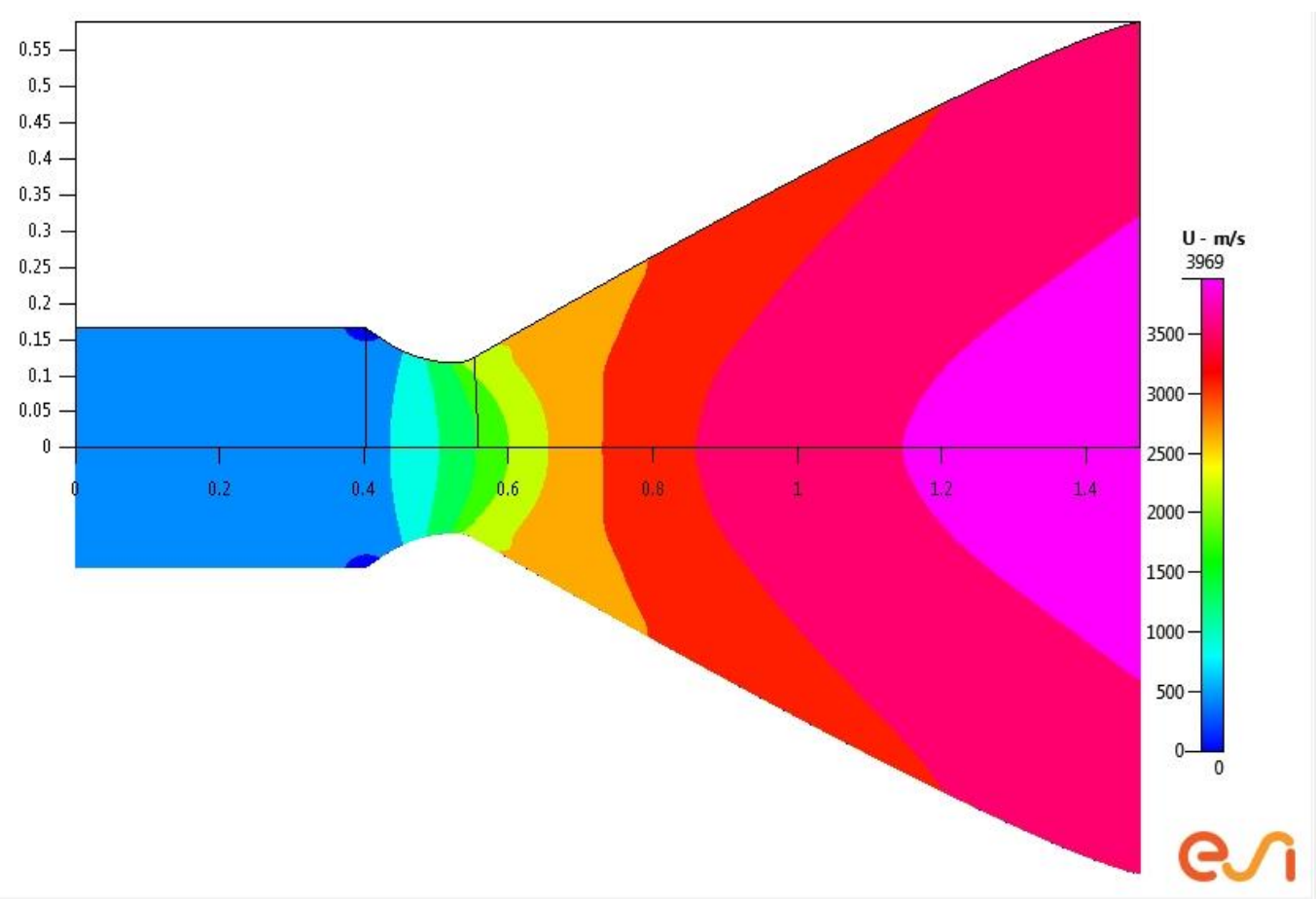

Figure 82 X - Direction Velocity Field Output for Stage 2 LOX/LH2

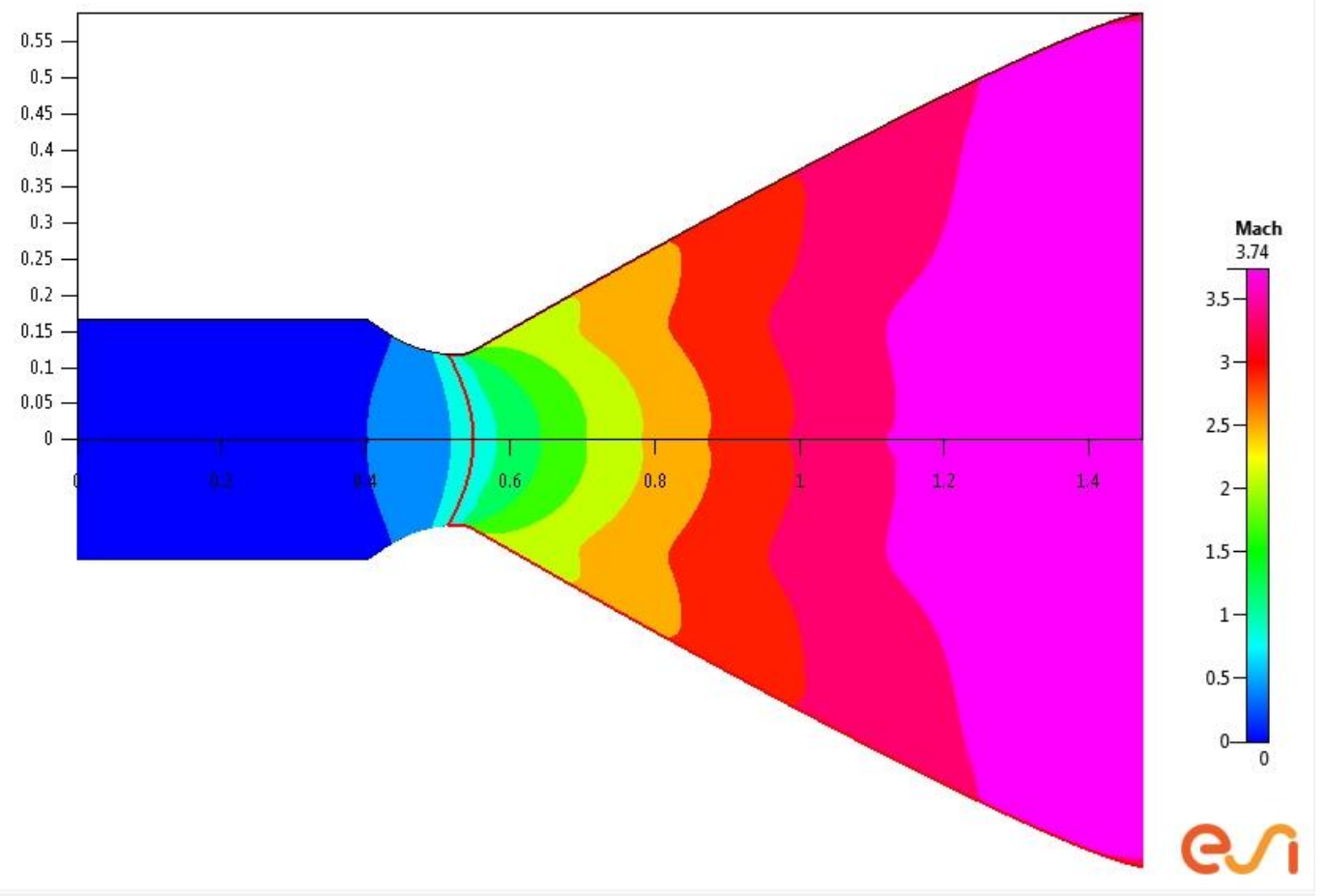

Figure 83 Mach Zones Output for Stage 2 LOX/LH2 
Stage 2 LH2 LF2 Output.

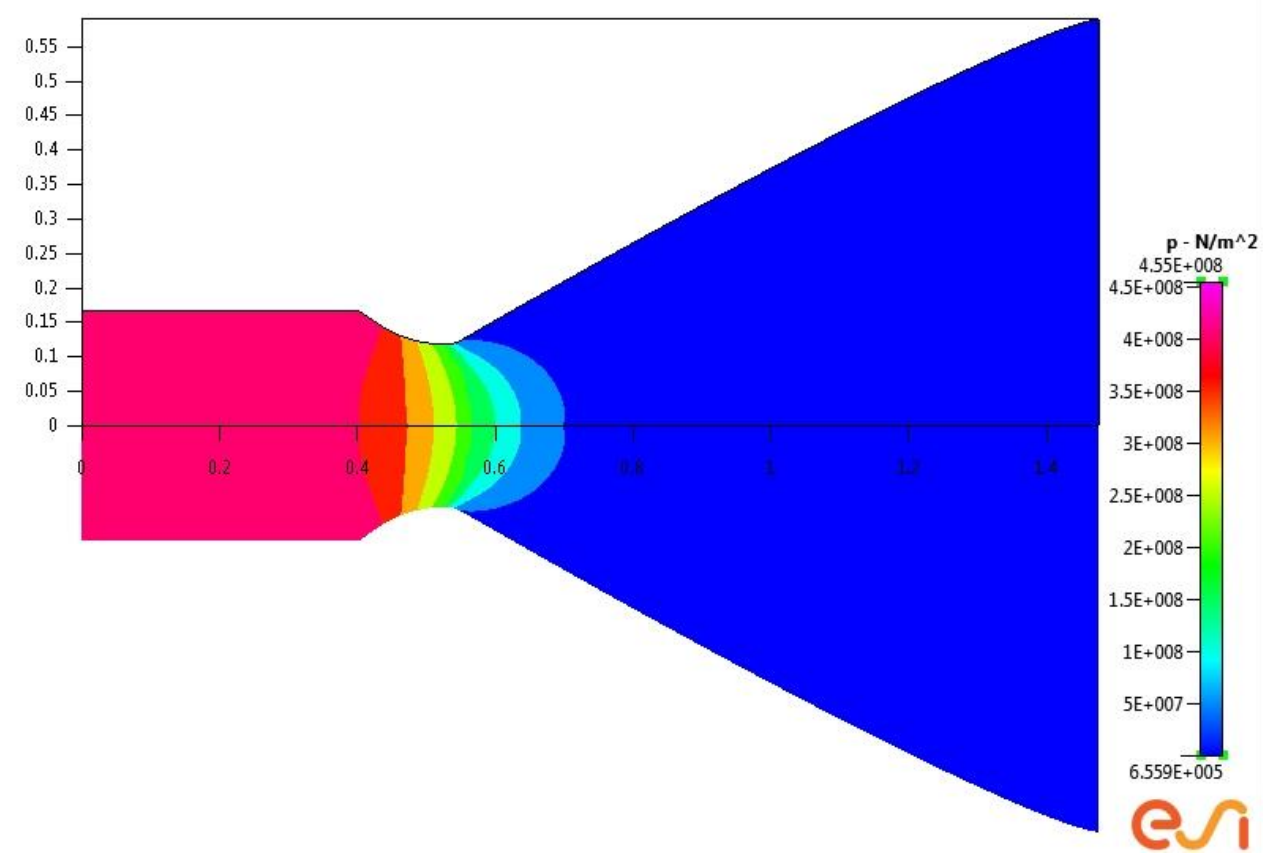

Figure 84 Pressure Map Output for Stage 2 LH2/LF2

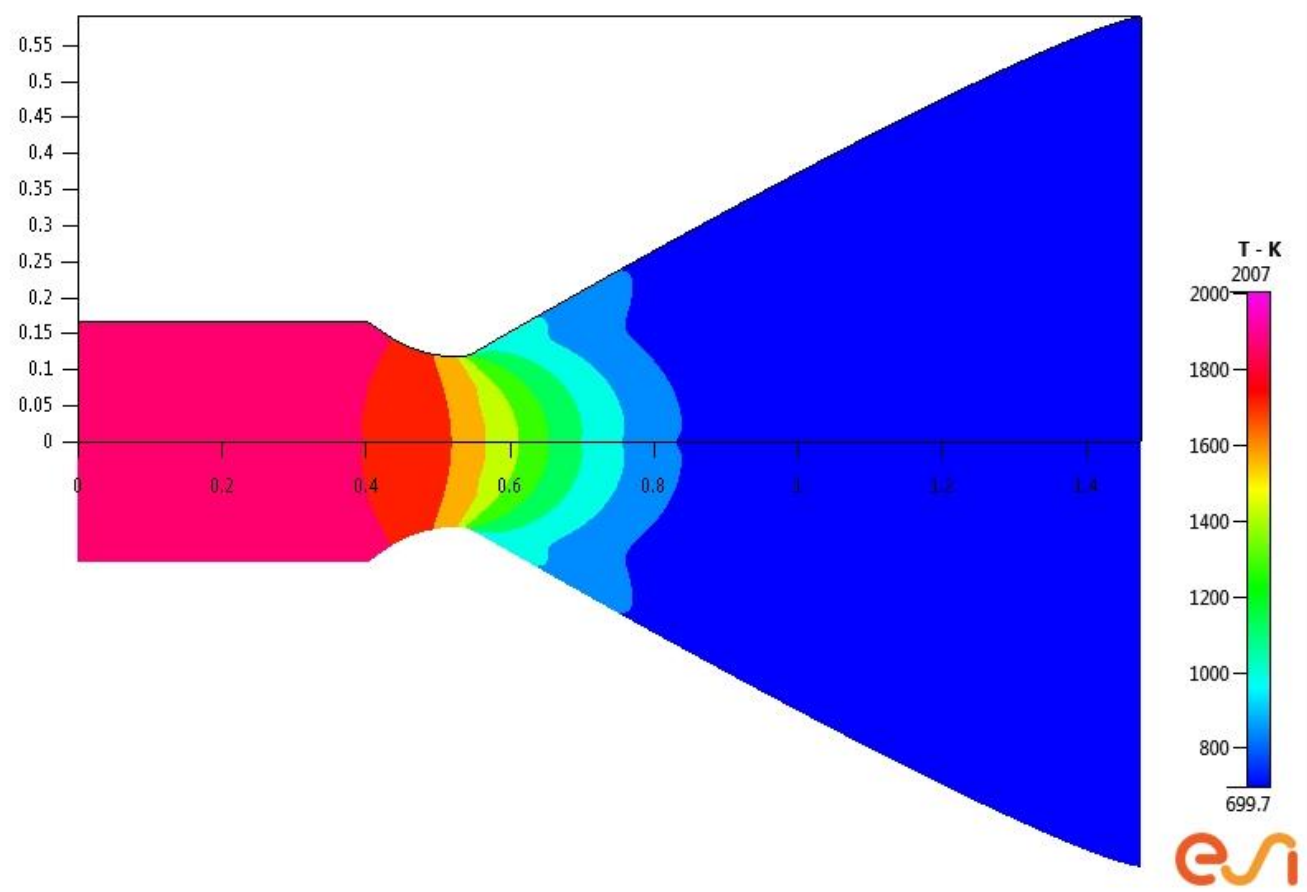

Figure 85 Temperature Map Output for Stage 2 LH2/LF2 


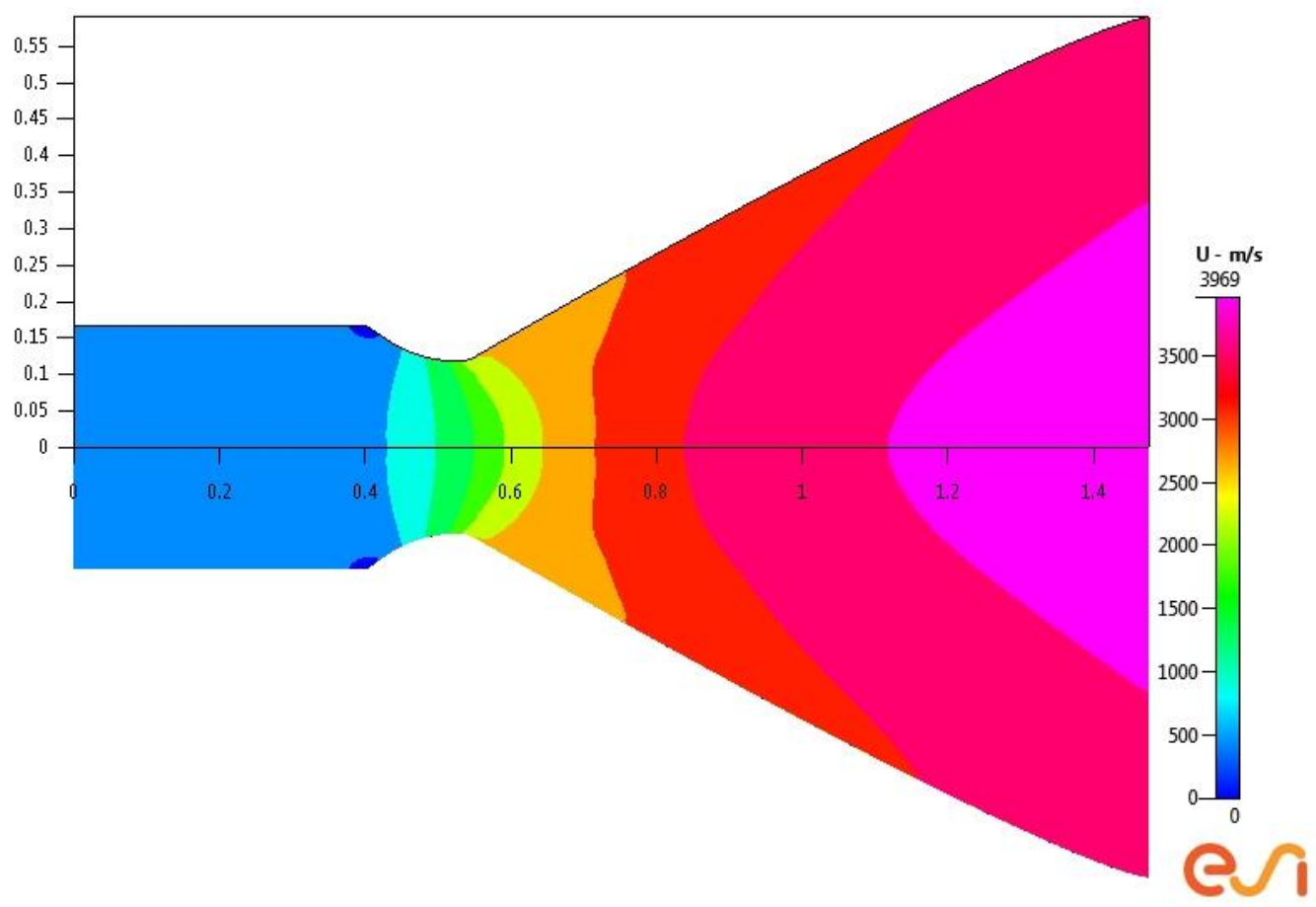

Figure 86 X - Direction Flow Field Output for Stage 2 LH2/LF2

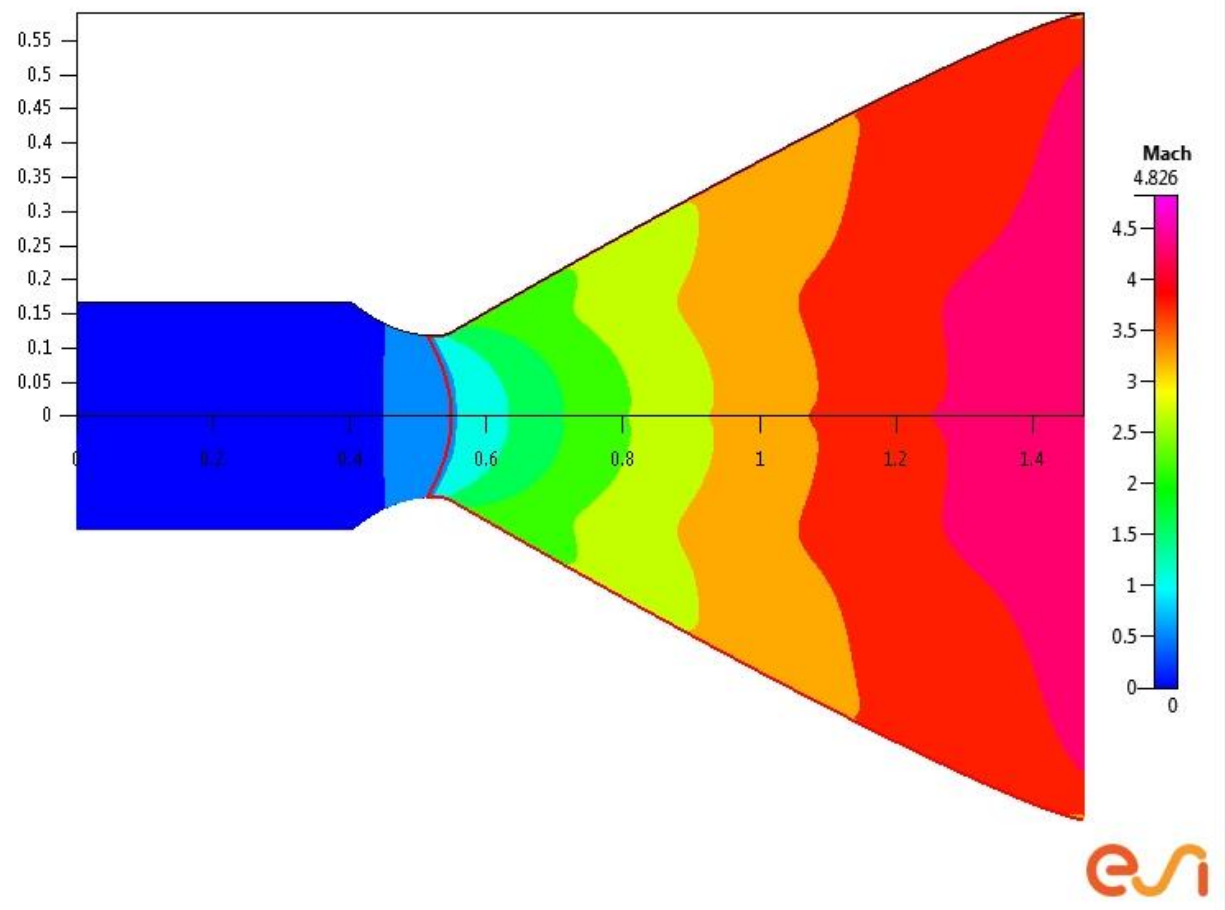

Figure 87 Mach Zones Output for Stage 2 LH2/LF2 


\section{Chapter 4 Conclusion}

This study developed results for modeling the propulsion systems of the Titan I and identifying methods of improvement. The geometry was generated from two sources and compared. The geometry of the original contour was, indeed, accurately detailed through engineering equations mentioned earlier to within a small percent error range. Then, using this geometry, the general flow field parameters--such as temperature, pressure, $\mathrm{x}$-velocity direction, and Mach zones--were modeled. This effort effectively created a benchmark of data that was not previously found in literature.

Once the original engine's combination and inputs were modeled and benchmarked, more inputs were run. Various runs and iterations were conducted using the original benchmarked geometry to suggest possible design improvements to the propulsion systems. The main inputs that drove the suggested improvements were the reactant combination and the pressure. The study ultimately shows that, depending on the required need of the vehicle, improvements for this propulsion system definitely exist. This implies that, if the engines need to have a higher thrust for the first stage system, then possible improvements include: (1) an increase the chamber pressure, and (2) a change in the propellant combination to liquid fluorine or liquid hydrogen. In order to have higher thrust in the second stage, keeping the original combination and inputs would be best according to the recorded data. Conversely, if the need was to have a more efficient vehicle, then the ISP would need to be raised. In order to have increased ISP in 
both the first and second stages, the propellants would need to be changed to liquid fluorine and liquid hydrogen, and then the pressure would need to be increased as well. In these cases, the pressure was increased from 587 psi and 682 psi for Stage 1 and 2 to 1000 psi.

The best upgrades would take into account any structural changes that would need to be done, which would affect overall vehicle performance. The flow results could help determine any anomalous operations such as increased vibration. Anomalous operations would need to be taken into account and would affect the overall structure of the engine assembly. However, this was outside of the scope of this study, as the focus was on solely on the propulsion systems. For the scope of this work, the best upgrade would be to increase the thrust as much as possible in order to complete the mission that this vehicle was originally intended for. In order to increase the thrust, the propellants for Stage 1 should be switched to liquid fluorine and liquid hydrogen, while for the second stage, the vehicle should remain as is because no advantage was observed in the study. Table 16 below displays the modified and improved engine parameters observed in this study. 


\section{Table 16 Titan I Proposed Improvement Parameters}

\begin{tabular}{|c|c|c|}
\hline \multicolumn{2}{|c|}{ Stage I Engine } & \multicolumn{2}{c|}{ Stage II Engine } \\
\hline Value & Parameter & Value \\
\hline 336318 & Sea Level Thrust (lb) & 72896 \\
\hline 2 & Number of Engines & 1 \\
\hline 2.25 & Mixture Ratio (O/F) & 2.25 \\
\hline 1000 & Chamber Pressure, psia & 682 \\
\hline 181.9 & Area Throat, in ${ }^{2}$ & 66.73 \\
\hline 8 & Area Ratio, Ae/At & 25 \\
\hline 382.46 & Sea Level Isp (sec) & 283 \\
\hline
\end{tabular}

Future improvements to this study could be made possible by scanning an existing Titan I propulsion system and to make a 3D model. This would greatly increase the quality of any CFD analysis, since the geometry would be identical to the actual system. Further improvements would require expanded research to include more propellant combinations, using even more inputs. An additional point of improvement could be to better-optimize the reactants to the geometry. Finally, further studies should eventually take into account the flow past the plane of the nozzle exit and analyze the plume impingement for further thrust or ISP propulsion improvements. 


\section{References}

Adams, L. C. (1990). Chapter 9 the evolution of the titan rocket - titan I to titan II. History of Rocketry and Astronautics: Proceedings of the Twenty-Fourth History Symposium of the International Academy of Astronautics, 19, 201-223.

Bird, G. A. (1994). Molecular gas dynamics and the direct simulation of gas flows (oxford engineering science). (2nd ed.). Oxford: Oxford University Press.

Brown, C. (1996). Spacecraft propulsion. (Education Series ed.). AIAA.

Cfd-fastran overview. (2011). ESI-Group.

Falcon 1 overview. (2012). Retrieved from http://www.spacex.com/falcon1.php

Gas generator rocket cycle. (2008). [Web Graphic]. Retrieved from http://en.wikipedia.org/wiki/File:Gas_generator_rocket_cycle.png

Hill, P. G., \& Peterson, C. R. (1992). Mechanics and thermodynamics of propulsion. (2nd ed.). New York, NY: Prentice Hall.

Houchin, R. F. (2006). US hypersonic research and development: The rise and fall of Dyna-Soar, 1944-1963. London: Routledge

Hunley, J. D. (2007). The development of propulsion technology for U.S. space-launch vehicles, 1926-1991. College Station: Texas A \& M University Press.

Huzel, D. K., Huang, D. H (1992). Modern engineering for design of liquid-propellant rocket engines. AIAA.

Launius, R. D. (2002). To reach the high frontier - a history of us launch vehicles. (1ed.). The University Press of Kentucky. 
Lange, O. H. (1963). Space carrier vehicles-design, development, and testing of launching rockets. New York: Academic Press.

Liepmann, H. W., Rothko, A., \& Lindsay, R. B. (1957). Elements of Gasdynamics. Physics Today. John Wiley \& Sons, Inc.

Lomax, H., Pulliam, T., Zingg, D., \& Kowalewski, T. (2002). Fundamentals of Computational Fluid Dynamics. Applied Mechanics Reviews, 55(4), B61. Springer.

Meland, L. C. (1989). Aiaa-89-2389 history of the titan liquid rocket engines. AIAA/ASME/SAE/ASEE 25th Joint Propulsion Conference

Murphy, J. M. (1980). The development of propulsion technology for us space launch vehicles, 1926-1991. History of rocketry and astronautics: proceedings of the twelfth, thirteenth, and fourteenth History Symposia of the International Academy of Astronautics, 10

NASA. (Artist). (1961). Dyna-Soar on Titan booster [Print Drawing]. Retrieved from http://nix.ksc.nasa.gov/info?id=MSFC-9902548\&orgid=11

Reber, L. (1986). Aiaa-86-1631 titan liquid engine propulsion past, present, and future.AIAA/ASME/ASEE 22nd Joint Propulsion Conference

Stumpf, D. K. (2000). Titan II: A History of a Cold War Missile Program. University of ARKANSAS Press. Retrieved from http://books.google.com/books?id=0ZjeIfgG2AoC

Zehe, M. (2010, February 03). Chemical equilibrium with applications. Retrieved from http://www.grc.nasa.gov/WWW/CEAWeb/ 


\section{Appendix}

\section{Sample CEA Output}

11118624.html

http://cearun.grc.nasa.gov/OFILES/11118624.html

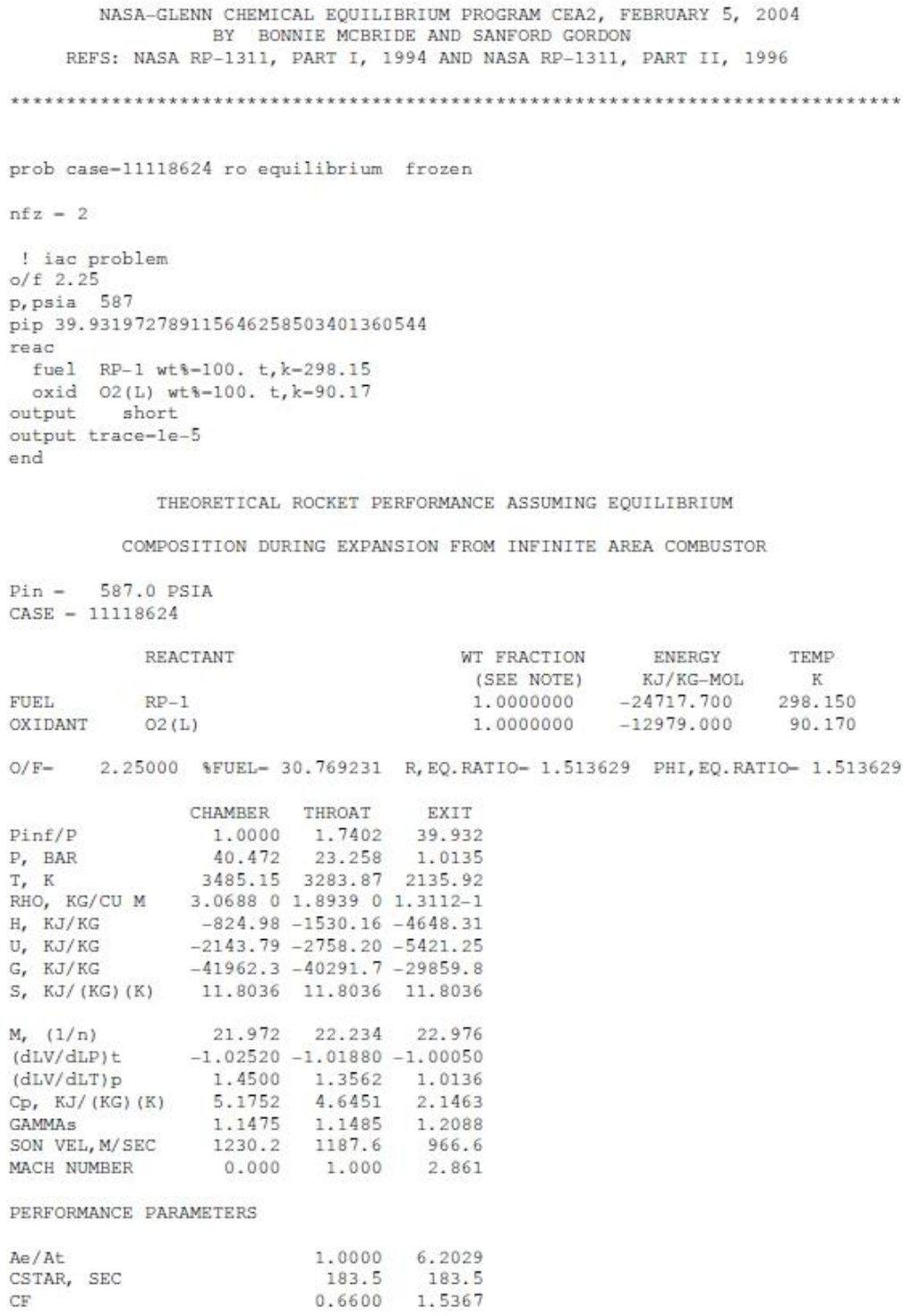

PERFORMANCE PARAMETERS

$\begin{array}{lrr}\text { Ae/At } & 1.0000 & 6.2029 \\ \text { CSTAR, SEC } & 183.5 & 183.5\end{array}$

$\begin{array}{lll}\text { CF } & 0.6600 & 1.5367\end{array}$




\section{Truncated Example Output of Forces}

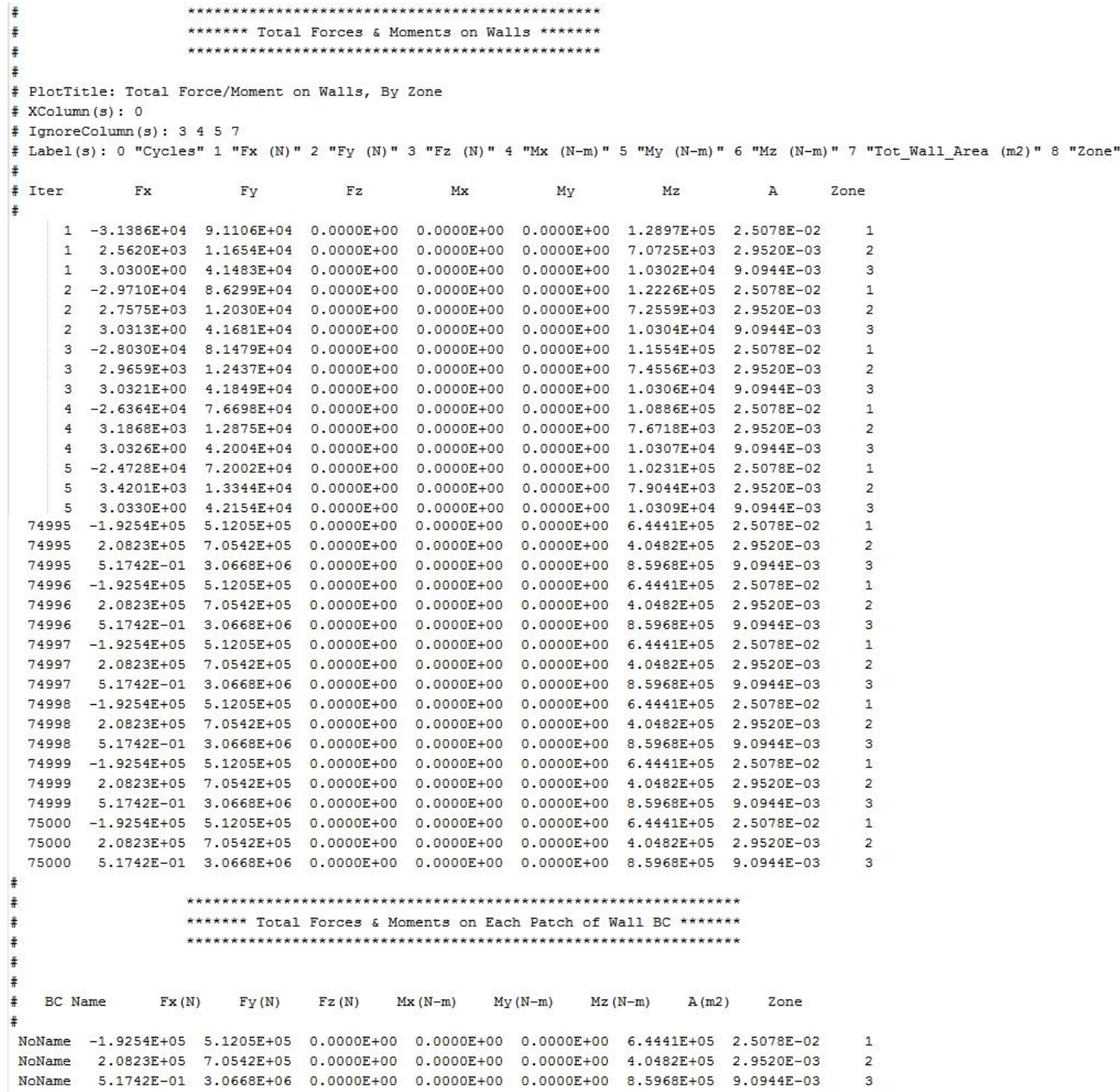




\section{EXCEL Spreadsheet Calculator Example for a Stage One Setup}

AIAA Modern Engineering for Design of Liquid-Propellant Rocket Engines (Huzel and Huang) Mechanics and Thermodynamics of Propulsion (Hill and Peterson)

\section{ONLY ENTER THESE VALUES}

Nozzle area expansion ratio:

Y Specific heat ratio

Pe

Chamber pressure

Exit pressure

€ Theoretical Nozzle area expansion ratio (AlAA p. 9)

Values Units
1.1485 N/A
587 psi
14.7 psi
6.53384

$\begin{array}{ll}\text { Values } & \text { Units } \\ \text { N/A } & \text { N/A }\end{array}$

$4047223 \mathrm{~Pa}$

$101353 \mathrm{~Pa}$

Coefficient of thrust:

Y Specific heat ratio

$\mathrm{Pe} \quad$ Exit pressure

Po Chamber pressure

Pa Ambient pressure

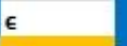

Nozzle area expansion ratio

Coefficient of thrust (AIAA p. 13)

Throat area:

Cf Coefficient of thrust

F Thrust

Po

At

Dt

Rt

Nozzle stagnation pressure

Throat area (AIAA p. 13)

Throat diameter

Throat radius

Exit diameter:

$\varepsilon$

Dt

De

De

$\mathrm{Ae}$

Contraction area ratio (AIAA p. 73)

Throat diameter

Exit diameter (AIAA p. 82)

Exit radius

Exit area

Chamber diameter:

ec Contraction area ratio

Dt Throat diameter

At $\quad$ Cross Sectional Area of the Throat

$\mathrm{L}^{*} \quad$ Characteristic Length (AIAA p.72)

Vc Volume of the Chamber (AIAA p.82)

DC Chamber diameter

Rc Chamber radius

Ac Cross Sectional Area of the Chamber

\section{Approximating Convergent Cone Length:}

Rt Throat radius

Contraction area ratio

R Radius of circular arc immediately upstream of throat (1.5Rt)

a Half-angle of conical section (range from 20-45 dea)

\begin{tabular}{|r|}
\hline 1.1485 \\
\hline $14.7 \mathrm{psi}$ \\
\hline $587 \mathrm{psi}$ \\
\hline $14.7 \mathrm{psi}$ \\
\hline 6.53384 \\
\hline 1.54597 \\
\hline
\end{tabular}

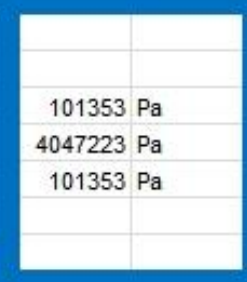

\begin{tabular}{|r|}
\hline 1.54597 \\
$150000 \mathrm{lbf}$ \\
587 psi \\
$165.292 \mathrm{in}^{\wedge} 2$ \\
14.5071 in \\
7.25356 in \\
\hline
\end{tabular}
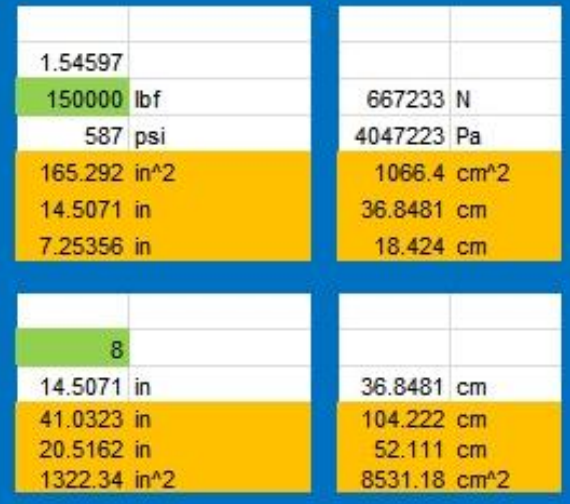

\begin{tabular}{|r|}
\hline 1.6 \\
14.5071 in \\
165.292 in \\
40 in \\
6611.68 in 3 \\
18.3502 in \\
9.1751 in \\
264.467 in \\
\hline
\end{tabular}

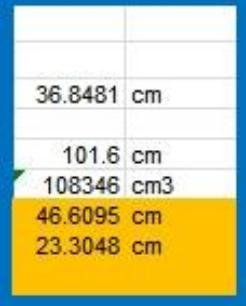

7.25356 in

6.53384

10.8803 in

$18.424 \mathrm{~cm}$

$27.636 \mathrm{~cm}$

$0.5236 \mathrm{rad}$ 


\begin{tabular}{|c|c|c|c|c|c|c|}
\hline \multicolumn{3}{|c|}{ x\% Bell Nozzle Configuration using Parabolic Approximation Procedure: } & & & & \\
\hline$\%$ & \multicolumn{2}{|c|}{ Percent of Bell Nozzle } & 80 & $\%$ & & \\
\hline Rt & \multicolumn{2}{|c|}{ Throat radius } & 7.25356 & in & 18.424 & $\mathrm{~cm}$ \\
\hline$\epsilon$ & \multicolumn{2}{|c|}{ Nozzle area expansion ratio } & 8 & & & \\
\hline$R$ & \multicolumn{2}{|c|}{ Divergent nozzle circular entrance section radius ( $0.382 R t)$} & 2.77086 & in & 7.03798 & $\mathrm{~cm}$ \\
\hline$\alpha$ & \multicolumn{2}{|c|}{ Half-angle of conical section (range from 12-18 deg) } & 0.27925 & $\mathrm{rad}$ & 16 & deg \\
\hline $\operatorname{Ln}$ & \multicolumn{2}{|c|}{ Nozzle length (AIAA p. 75 ) } & 37.3133 & in & 94.7757 & $\mathrm{~cm}$ \\
\hline \multicolumn{7}{|c|}{ Characteristic velocity: } \\
\hline 9 & Gravity & & 32.2 & $\mathrm{ft} / \mathrm{s}^{\wedge} 2$ & & \\
\hline$\checkmark$ & Specific heat ratio & & 1.1485 & & 1.1485 & \\
\hline M & Molecular weight & & 22.976 & slug/lbmol & 22.976 & $\mathrm{~g} / \mathrm{mol}$ \\
\hline Rbar/M & Gas constant & & 67.2006 & $\mathrm{ft}^{*} \mid \mathrm{b} f /$ slug $^{*} \mathrm{R}$ & 361.876 & $\mathrm{~J} / \mathrm{kgK}$ \\
\hline To & \multicolumn{2}{|c|}{ Chamber total temperature } & 6312.6 & $R$ & 3507 & $\mathrm{~K}$ \\
\hline$c^{*}$ & \multicolumn{2}{|c|}{ Theoretical characteristic velocity (AIAA p. 13) } & 5789.88 & $\mathrm{ft} / \mathrm{s}$ & 1764.81 & $\mathrm{~m} / \mathrm{s}$ \\
\hline \multicolumn{7}{|c|}{ Mass flow rate: } \\
\hline $\mathrm{F}$ & Thrust & & & & 667233 & $N$ \\
\hline$c^{*}$ & \multicolumn{2}{|c|}{ Characteristic velocity } & & & 1764.81 & $\mathrm{~m} / \mathrm{s}$ \\
\hline $\mathrm{Cf}$ & \multicolumn{2}{|c|}{ Coefficient of thrust } & & & 1.54597 & \\
\hline mdot & \multicolumn{2}{|c|}{ Mass flow rate (Hill and Peterson p.518) } & & & 244.555 & $\mathrm{~kg} / \mathrm{s}$ \\
\hline \multicolumn{7}{|c|}{ Weight flow rate: } \\
\hline At & Throat area & & 165.292 & in $^{\wedge} 2$ & & \\
\hline Po & Chamber pressure & & 587 & psi & & \\
\hline$g$ & Gravity & & 32.2 & $\mathrm{ft} / \mathrm{s}^{\wedge} 2$ & & \\
\hline 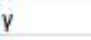 & Specific heat ratio & & 1.1485 & & & \\
\hline $\mathrm{Rbar} / \mathrm{M}$ & Gas Constant & & 67.2006 & $\mathrm{ft}^{*} \mathrm{~b}$ f $/$ slug ${ }^{*} R$ & & \\
\hline To & \multicolumn{2}{|c|}{ Chamber temperature } & 6312.6 & $\mathrm{R}$ & & \\
\hline Wdot & Weight flow rate ( $A$ & AA p. 9) & 539.605 & $\mathrm{lbf} / \mathrm{s}$ & & \\
\hline Thrust $\mathrm{cl}$ & ber specific impulse & & & & & \\
\hline $\mathrm{F}$ & Thrust & & 150000 & lbf & & \\
\hline Wdot & Weight flow rate & & 539.605 & $\mathrm{lb} / \mathrm{s}$ & & \\
\hline is & Specific impulse (A & (A.p. 12) & 277.981 & $s$ & 2.72835 & $\mathrm{kN}^{*} \mathrm{~s} / \mathrm{kc}$ \\
\hline Exit velo & (metric): & & & & & \\
\hline $\mathrm{Pe}$ & Exit pressure & & & & 101353 & $\mathrm{~Pa}$ \\
\hline $\mathrm{Pa}$ & Ambient pressure & & & & 101353 & $\mathrm{~Pa}$ \\
\hline $\mathrm{Ae}$ & Exit area & & & & 0.85312 & $m^{\wedge} 2$ \\
\hline mdot & Mass flow rate & & & & 244.555 & $\mathrm{~kg} / \mathrm{s}$ \\
\hline $\mathrm{F}$ & Thrust & & & & 667233 & $\mathrm{~N}$ \\
\hline Ve & Exit velocity (Hill an & Peterson p. 516) & & & 2728.35 & $\mathrm{~m} / \mathrm{s}$ \\
\hline Exit velo & (English): & & & & & \\
\hline g & Gravity & & 32.2 & $\mathrm{ft} / \mathrm{s}^{\wedge} 2$ & & \\
\hline$\gamma$ & Specific heat ratio & & 1.1485 & & & \\
\hline $\mathrm{Rbar} / \mathrm{M}$ & Gas constant & & 67.2006 & $\mathrm{ft}^{*} \mathrm{~b}$ f/slug*R & & \\
\hline To & Chamber temperatu & & 6312.6 & $R$ & & \\
\hline $\mathrm{Pe}$ & Exit pressure & & 14.7 & psi & & \\
\hline Po & Chamber pressure & & 587 & psi & & \\
\hline Ve & Fxit velncitv (AIAA & & 8.950 .98 & $\mathrm{ft} / \mathrm{s}$ & 272826 & $\mathrm{~m} / \mathrm{s}$ \\
\hline
\end{tabular}




\begin{tabular}{|c|c|c|c|c|c|c|}
\hline \multicolumn{7}{|c|}{ Pressure at throat: } \\
\hline Po & Chamber pressure & & 587 & psi & & \\
\hline$\gamma$ & Specific heat ratio & & 1.1485 & & & \\
\hline $\mathrm{Pt}$ & \multicolumn{2}{|c|}{ Pressure at throat (AIAA p.9) } & 337.341 & psi & \multicolumn{2}{|c|}{$2325884 \mathrm{~Pa}$} \\
\hline \multicolumn{7}{|c|}{ Velocity at throat: } \\
\hline 9 & Gravity & & 32.2 & $\mathrm{ft} / \mathrm{s}^{\wedge} 2$ & & \\
\hline$v$ & Specific heat ratio & & 1.1485 & & & \\
\hline $\mathrm{Rbar} / \mathrm{M}$ & Gas constant & & 67.2006 & $\mathrm{ft}^{*} \mid \mathrm{b} f /$ slug ${ }^{*} \mathrm{R}$ & & \\
\hline To & \multicolumn{2}{|c|}{ Chamber temperature } & 6312.6 & $\mathrm{R}$ & & \\
\hline Vt & \multicolumn{2}{|c|}{ Velocity at throat (AIAA p.9) } & 3821.48 & $\mathrm{ft} / \mathrm{s}$ & \multicolumn{2}{|c|}{$1164.79 \mathrm{~m} / \mathrm{s}$} \\
\hline \multicolumn{7}{|c|}{ Approximate chamber length: } \\
\hline Dt & Throat diameter & & 14.5071 & in & 36.8481 & $\mathrm{~cm}$ \\
\hline Lc 1 & \multicolumn{2}{|c|}{ Chamber length using trial 1} & 22.5767 & in & 57.3449 & \\
\hline Lc 2 & \multicolumn{2}{|c|}{ Chamber length using trial 2} & 21.9821 & in & 55.8344 & \\
\hline Lc & \multicolumn{2}{|c|}{ Chamber length } & 22.2794 & in & 56.5897 & $\mathrm{~cm}$ \\
\hline Lc & \multirow{2}{*}{\multicolumn{2}{|c|}{$\begin{array}{l}\text { Chamber length (http://www.braeunig.us/space/propuls.htm) } \\
\% \text { difference between calc length and Braeunig's }\end{array}$}} & 21.7648 & in & 55.2827 & $\mathrm{~cm}$ \\
\hline$\%$ & & & 2.30963 & \% difference between calc length and Braeunig's & & \\
\hline \multicolumn{7}{|c|}{ Approximate chamber volume: } \\
\hline At & \multicolumn{2}{|l|}{ Throat area } & 165.292 & in $^{\wedge} 2$ & 1066.4 & $\mathrm{~cm}^{\wedge} 2$ \\
\hline Lc & \multirow{2}{*}{\multicolumn{2}{|c|}{$\begin{array}{l}\text { Chamber length } \\
\text { Angle of convergent section }\end{array}$}} & 22.2794 & in & 56.5897 & $\mathrm{~cm}$ \\
\hline$\theta$ & & & 0.5236 & rad & 30 & $\operatorname{deg}$ \\
\hline$\varepsilon$ & \multicolumn{2}{|c|}{ Chamber contraction area ratio } & 1.6 & & & \\
\hline Ve & \multicolumn{2}{|c|}{ Chamber volume (AIAA p. 74) } & 6009.57 & $\mathrm{in}^{\wedge} 3$ & 98479.3 & $\mathrm{~cm}^{\wedge} 3$ \\
\hline \multicolumn{7}{|c|}{ Convergent cone volume: } \\
\hline Lc & \multicolumn{2}{|c|}{ Convergent cone length } & 22.4659 & in & 57.0635 & $\mathrm{~cm}$ \\
\hline Rc & \multicolumn{2}{|c|}{ Chamber radius } & 9.1751 & in & 23.3048 & $\mathrm{~cm}$ \\
\hline Rt & \multicolumn{2}{|c|}{ Throat radius } & 7.25356 & in & 18.424 & $\mathrm{~cm}$ \\
\hline Vcc & \multicolumn{2}{|c|}{ Convergent cone volume } & 3218.32 & in $^{\wedge} 3$ & 52738.7 & $\mathrm{~cm}^{\wedge} 3$ \\
\hline Volume & cylindrical chamber & ction: & & & & \\
\hline Vc & Chamber volume & & 6009.57 & in $^{\wedge} 3$ & 98479.3 & $\mathrm{~cm}^{\wedge} 3$ \\
\hline Vcc & Convergent cone v & ume & 3218.32 & in $^{\wedge} 3$ & 52738.7 & $\mathrm{~cm}^{\wedge} 3$ \\
\hline Vcs & Volume for cylindric & I section & 2791.26 & $\operatorname{in}^{\wedge} 3$ & 45740.5 & $\mathrm{~cm}^{\wedge} 3$ \\
\hline Length $\mathrm{fc}$ & ylindrical chamber s & tion: & & & & \\
\hline Ves & Volume for cylindric & I section & 2791.26 & $\operatorname{in}^{\wedge} 3$ & 45740.5 & $\mathrm{~cm}^{\wedge} 3$ \\
\hline$\varepsilon$ & Chamber contractio & area ratio & 1.6 & & & \\
\hline At & Throat area & & 165.292 & in $^{\wedge} 2$ & 1066.4 & $\mathrm{~cm}^{\wedge} 2$ \\
\hline LCs & Cylindrical section I & igth & 10.5543 & in & 26.8079 & $\mathrm{~cm}$ \\
\hline
\end{tabular}

